\title{
Performance Assessment of RC Columns Under Near-Field Blast Loading Using CFD Modelling
}

\author{
by \\ Riana Vidjen
}

A thesis submitted to the Faculty of Graduate and Postdoctoral Affairs in partial fulfillment of the requirements for the degree of

\author{
Master of Applied Science \\ in
}

Civil Engineering

Carleton University

Ottawa, Ontario

(C) 2021

Riana Vidjen 


\begin{abstract}
This study investigates the effects of various design parameters on the performance of reinforced concrete columns under near-field blast loads, specifically for scaled distances less than 0.4 $\mathrm{m} / \mathrm{kg}^{1 / 3}$. The computational fluid dynamics analysis method in LS-DYNA is used to model the detonation process of the explosive, the propagation of the blast wave, and its interaction with the structure. The model's ability to accurately predict blast loads and simulate structural response is verified against experimental data from the literature. Using the verified model, the influence of transverse reinforcement spacing, concrete cover, axial load ratio, and column cross-section shape on the structural performance is evaluated based on several parameters including the lateral displacement, the extent of the damage zone, material stress condition, and residual axial capacity. Based on the analysis results, it is concluded that a reduction in the transverse reinforcement spacing reduces the lateral displacement and spall length, while increasing the residual axial capacity. Also, a reduction in concrete cover is found to reduce spalling but has a minimal effect on the lateral displacements. Lastly, it is shown that increasing the axial load ratio significantly reduce the lateral displacement, but past a certain point can lead to shear failure near the support.
\end{abstract}




\section{Acknowledgements}

I would like to express my gratitude towards my supervisors Dr. Vahid Sadeghian and Dr. Murat Saatcioglu. Thank you for providing me the opportunity to complete this research project under your supervision. Your combined guidance and invaluable expertise were instrumental in this work.

I would also like to thank Dr. Alok Dua for his assistance in this project. Your knowledge and constructive feedback, particularly in regard to LS-DYNA, were greatly appreciated.

Finally, I would like to thank my family for their encouragement and support during my studies, especially my parents, who provided me a strong foundation for my achievements. 


\section{Table of Contents}

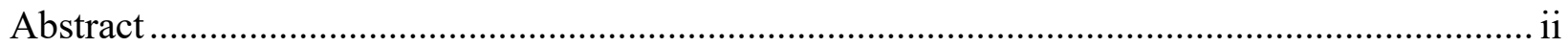

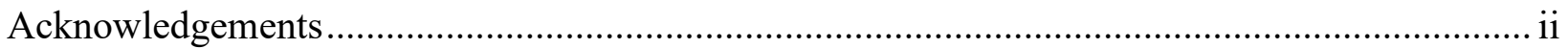

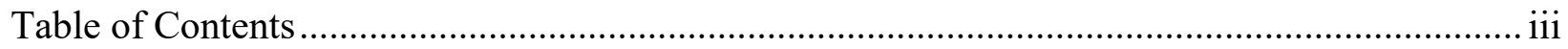

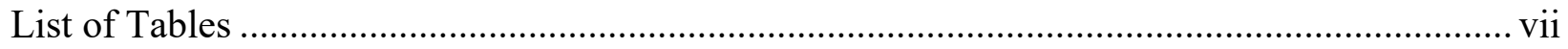

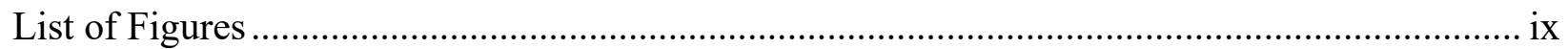

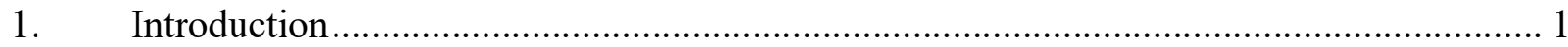

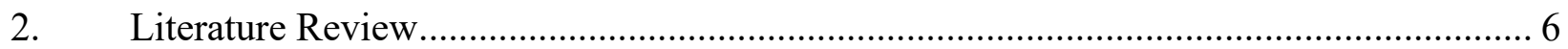

2.1. Simplified Blast Models ................................................................................. 6

2.2. Computational Fluid Dynamics Models ………………………........................ 11

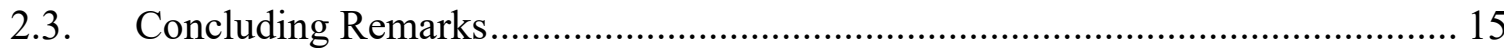

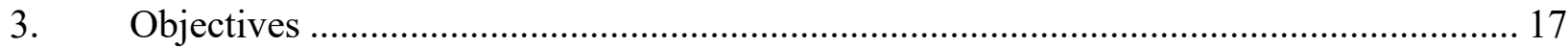

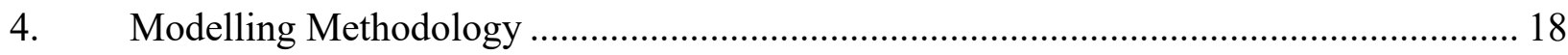

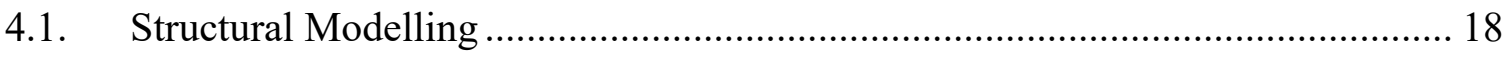

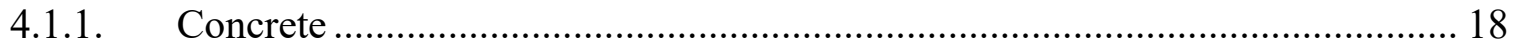

4.1.1.1. Material Model............................................................................................. 18

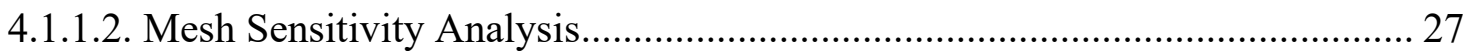

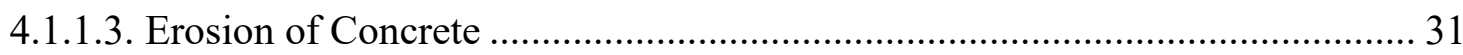

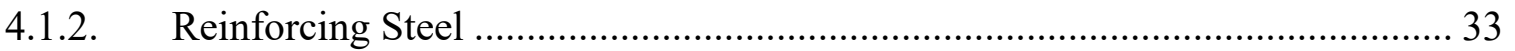

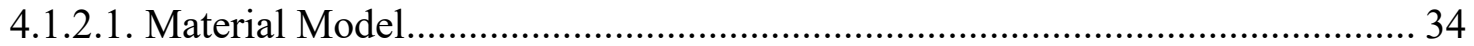


4.1.2. Mesh Sensitivity Analysis.

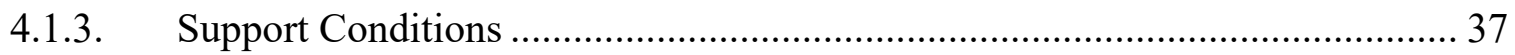

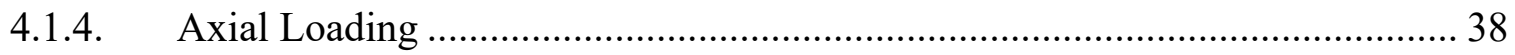

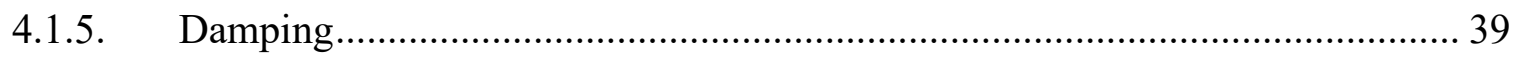

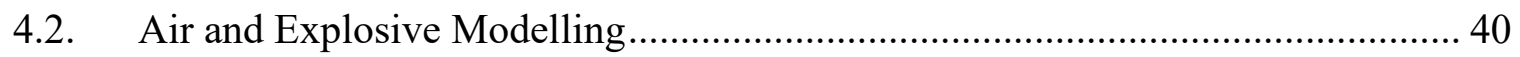

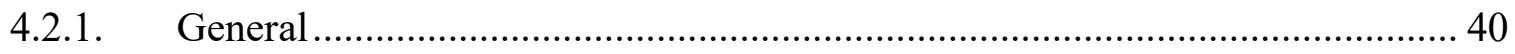

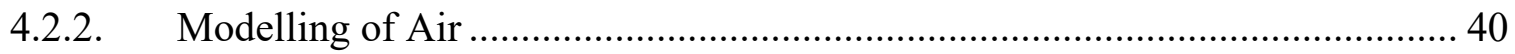

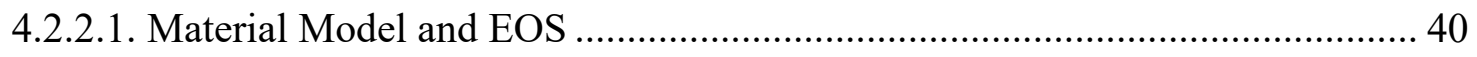

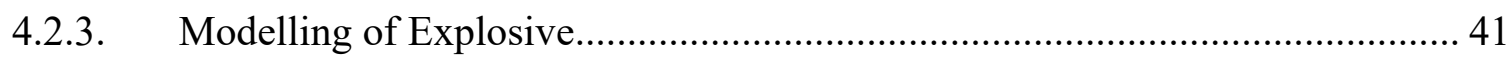

4.2.3.1. Material Model and EOS .................................................................... 41

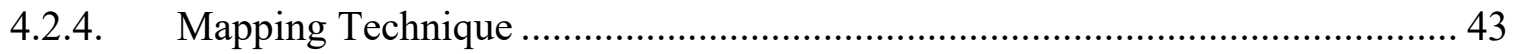

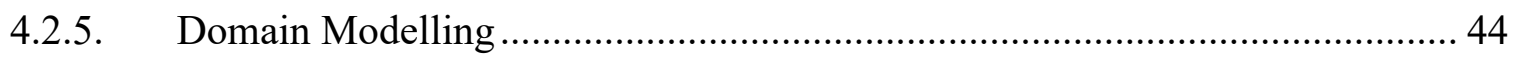

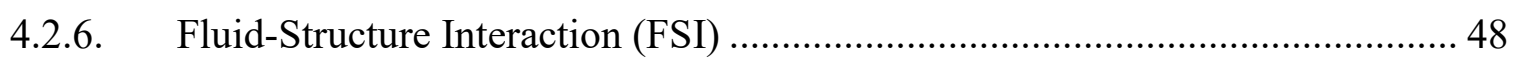

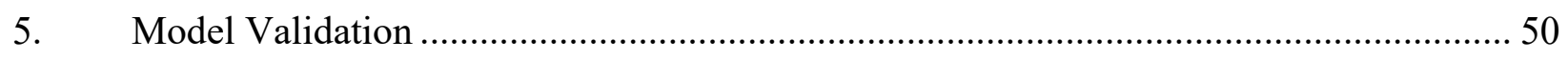

5.1. Validation of Blast Wave Propagation Modelling in Near-Field ....................... 51

5.1.1. Validation against Rigby et al. (2014) Tests............................................ 51

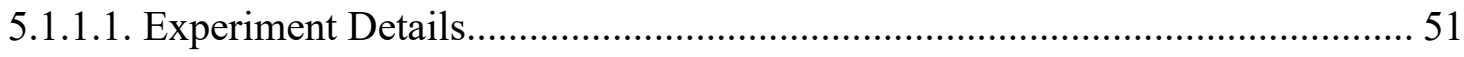

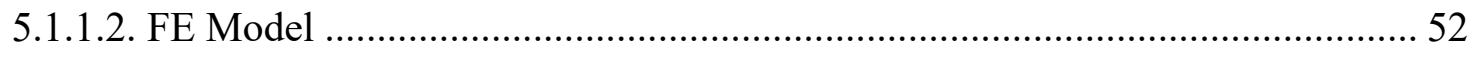

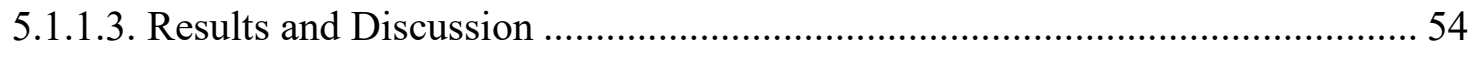

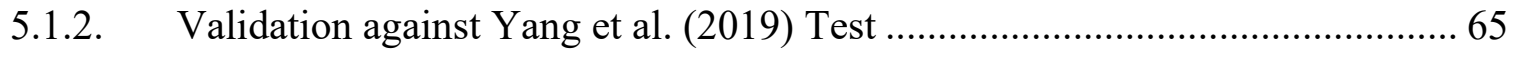


5.1.2.1. Experiment Details 65

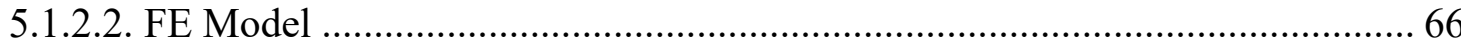

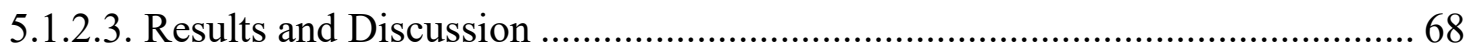

5.2. Validation of Structural Response under Near-Field Blast Loading ................... 70

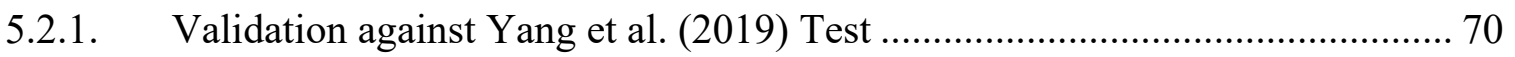

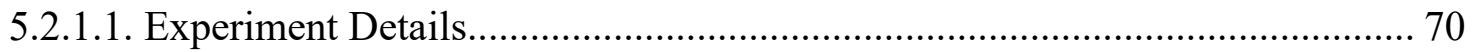

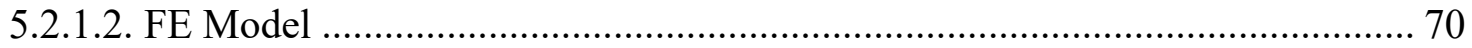

5.2.1.3. Results and Discussion .................................................................... 71

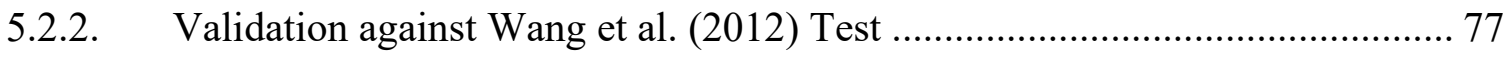

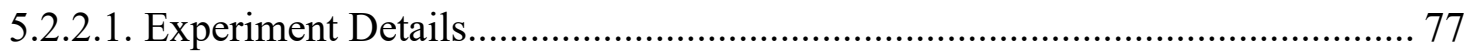

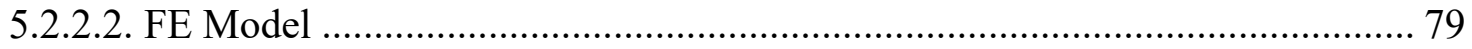

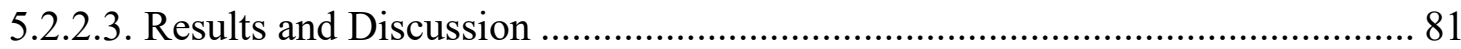

5.2.3. Validation against Zhang et al. (2013) Test.............................................. 90

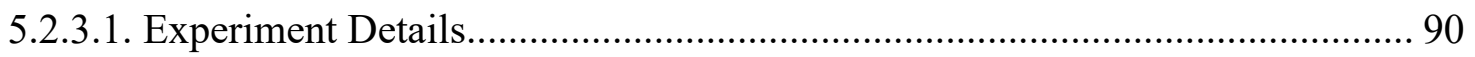

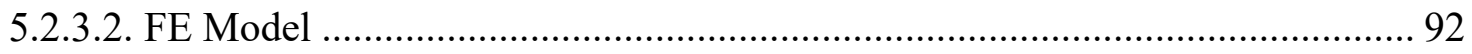

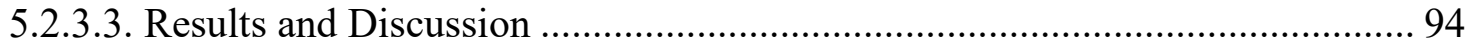

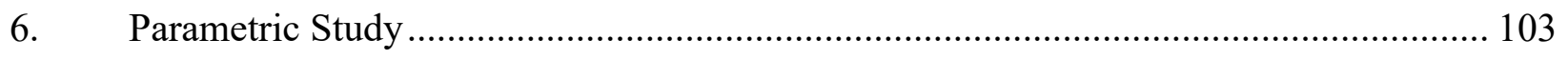

6.1. Numerical Model ................................................................................. 103

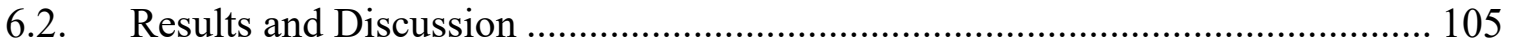

6.2.1. Effect of Transverse Reinforcement ..................................................... 105 


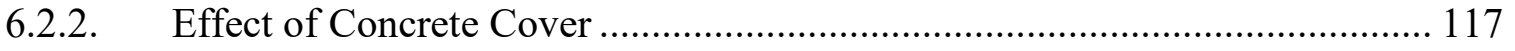

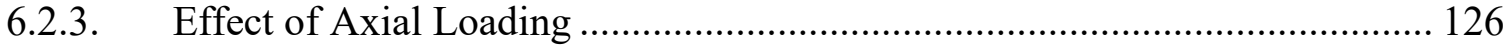

6.2.4. Effect of Column Shape ........................................................................ 136

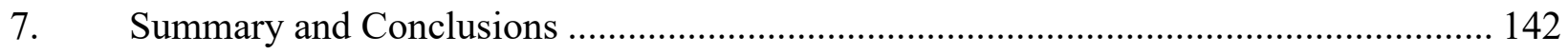

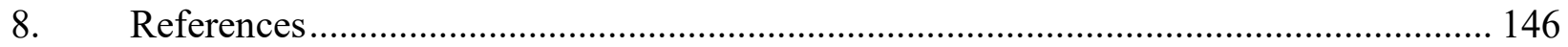




\section{List of Tables}

Table 1: Concrete mesh sensitivity analysis material properties. .......................................... 28

Table 2: Analysis times for various mesh sizes of solid concrete elements using CSCM. ......... 30

Table 3: Analysis times for various mesh sizes of beam elements using

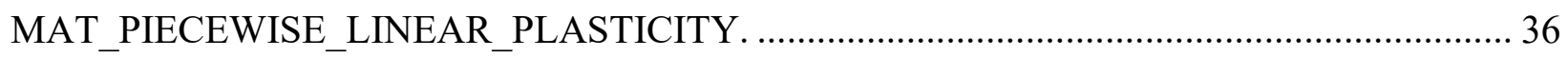

Table 4: Material and EOS parameters for air............................................................. 41

Table 5: Material parameters for explosives.............................................................. 42

Table 6: EOS parameters for explosives....................................................................... 42

Table 7: Analysis times and total number of elements for various 2D air mesh sizes. ............. 46

Table 8: Comparison of peak and average reflected pressures of all bars for all tests to FE model

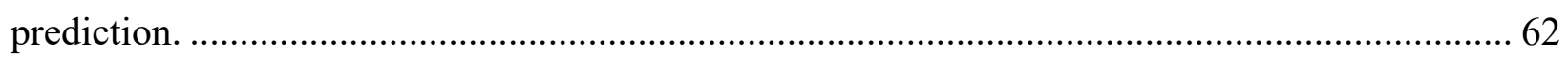

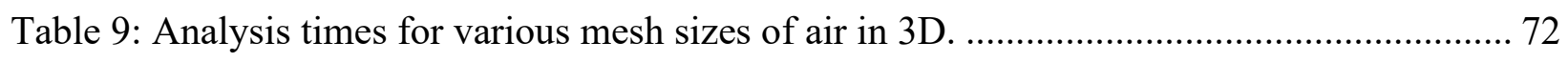

Table 10: Comparison of damage between experiment and FE model. .................................. 97

Table 11: Axial strain distribution in steel reinforcement at peak response for varying amount of

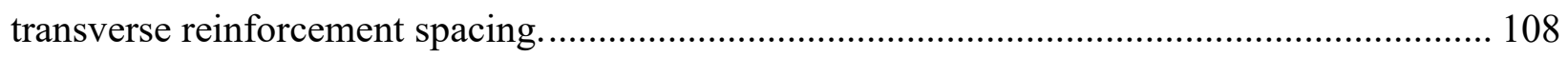

Table 12: Residual effective strain distribution in blast-damaged columns for varying amount of

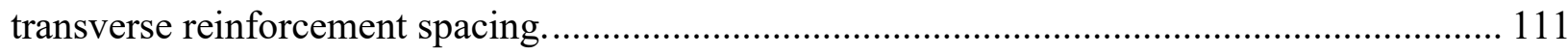

Table 13: Effective stress distribution in columns at peak response for varying amount of

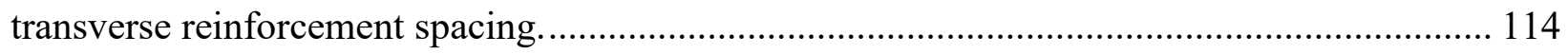
Table 14: Effect of confinement on compressive strength, compressive strain, and axial capacity of columns with varying transverse reinforcement spacing. ............................................. 115 Table 15: Residual effective strain distribution in blast-damaged columns for varying amount of concrete cover. 120 
Table 16: Axial strain distribution in steel reinforcement at peak response for varying amount of concrete cover.

Table 17: Effective stress distribution in columns at peak response for varying amount of concrete cover.

Table 18: Effect of confinement on compressive strength, compressive strain, and axial capacity of columns with varying concrete cover. 125

Table 19: Residual effective strain distribution in blast-damaged columns for varying axial load ratios.

Table 20: Axial strain distribution in steel reinforcement at peak response for varying axial load ratios. 130

Table 21: Effective stress distribution in columns at peak response for varying axial load ratios.

Table 22: Effective strain distribution in blast-damaged columns for various cross-section shapes.

Table 23: Axial strain distribution in steel reinforcement at peak response for various crosssection shapes. 


\section{List of Figures}

Figure 1: (a) Near-field and (b) far-field blast loading on a structure. ..................................... 2

Figure 2: Global failure of RC columns under blast as a result of (a) flexural failure, (b) shear

failure, and (c) flexural-shear failure. .......................................................................... 3

Figure 3: Local failure of RC columns under blast as a result of (a) cratering and spalling and (b)

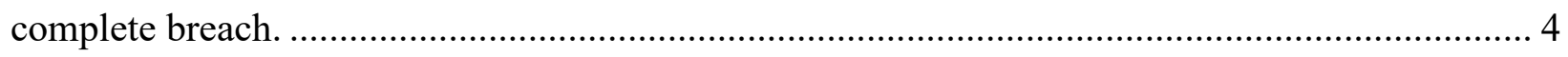

Figure 4: Damage profile of RC slab tested by Wang et al. (2012) obtained from: (a) experiment, (b) Winfrith concrete model, (c) KCC concrete model, and (d) CSCM concrete model. The incident face is shown in the left and the distal face on the right. 20

Figure 5: CSCM yield surface in (a) three dimensions and (b) two dimensions (Murray, 2007). 24

Figure 6: DIF in tension and compression for CSCM concrete (Murray, 2007)...................... 25

Figure 7: Uniaxial stress-strain curves of concrete with unconfined compressive strength of 30

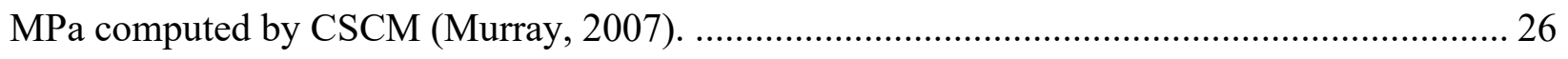

Figure 8: Results of a triaxial compression test on concrete using CSCM (Murray, 2004)........ 26

Figure 9: Detailing of RC column used in concrete mesh sensitivity analysis......................... 28

Figure 10: Mesh sizes of solid concrete elements for the RC column used in the sensitivity

analysis: (a) $100 \mathrm{~mm}$ (b) $75 \mathrm{~mm}$ (c) $50 \mathrm{~mm}$ (d) $25 \mathrm{~mm}$ (e) $20 \mathrm{~mm}$ (f) $15 \mathrm{~mm}$ (g) $10 \mathrm{~mm} \ldots \ldots \ldots . . .29$

Figure 11: Mesh sensitivity results for axially loaded RC column using CSCM concrete. ......... 30

Figure 12: Analysis time and total number of elements for various concrete solid element mesh

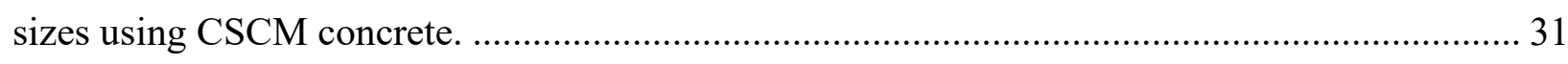

Figure 13: Quarter model of an RC slab showing erosion of concrete elements (a) prior to blast

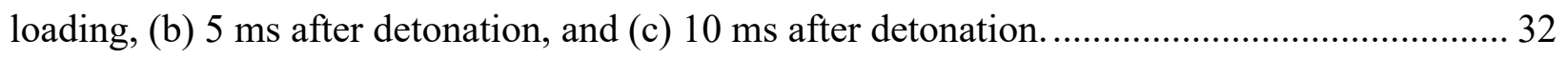

Figure 14: Maximum principal strains for elements deleted by erosion. ................................. 32 
Figure 15: Stress-strain relationship for steel under static and rapid loading (UFC 03-0340-02, 2008).

Figure 16: Mesh sensitivity results for beam elements representing steel reinforcing bars using PIECEWISE_LINEAR_PLASTICITY material model. 36

Figure 17: Analysis times and total number of elements for various beam element mesh sizes using MAT_PIECEWISE_LINEAR_PLASTICITY. 36

Figure 18: Interaction between two Lagrangian entities (steel supports and an RC slab) using CONTACT_AUTOMATIC_SURFACE_TO_SURFACE (a) prior to loading application and (b) after loading application. 38

Figure 19: Application of axial loading to RC columns. 39

Figure 20: (a) Air and explosive in the 2D model (b) Blast pressure propagation in 2D. 44

Figure 21: Mesh sensitivity analysis of overpressures generated during the detonation process. 45 Figure 22: Mesh sensitivity analysis of the impulse generated during the detonation process... 45 Figure 23: Analysis time and total number of elements for various 2D air mesh sizes. 46

Figure 24: (a) 3D model of air and explosive (b) Blast pressure propagation in 3D................. 47 Figure 25: Coupling of ALE material to a Lagrangian plate showing (a) poor coupling with leakage and (b) proper coupling and no leakage.

Figure 26: Test arrangement used to collect overpressure-time histories. (a) Schematic showing overall test setup and (b) Hopkinson bar configuration (Rigby et al., 2015)..... 52 Figure 27: (a) 2D FE model of Rigby et al. (2015) (b) Propagation of blast pressures just prior to mapping. 53

Figure 28: (a) 3D FE model of Rigby et al. (2015) (b) Propagation of blast pressures just after mapping. 53 
Figure 29: Raw experimental pressure data for the central bar (Bar 5) (Rigby et al., 2015)....... 55

Figure 30: Comparison of FE model prediction for central bar (Bar 5) against maximum and

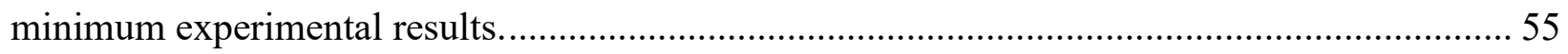

Figure 31: Raw experimental pressure data for a radial bar (Bar 1) (Rigby et al., 2015). .......... 56

Figure 32: Comparison of FE model prediction for radial bar against maximum and minimum

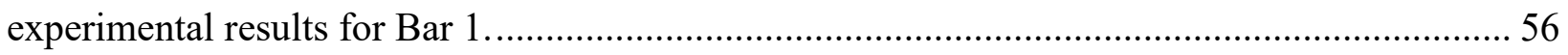

Figure 33: Raw experimental pressure data for a radial bar (Bar 2) (Rigby et al., 2015). .......... 56

Figure 34: Comparison of FE model prediction for radial bar against maximum and minimum

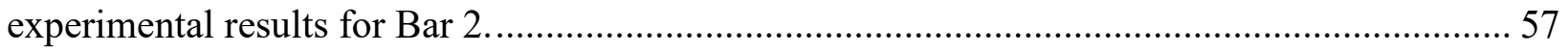

Figure 35: Raw experimental pressure data for a radial bar (Bar 3) (Rigby et al., 2015). .......... 57

Figure 36: Comparison of FE model prediction for radial bar against maximum and minimum

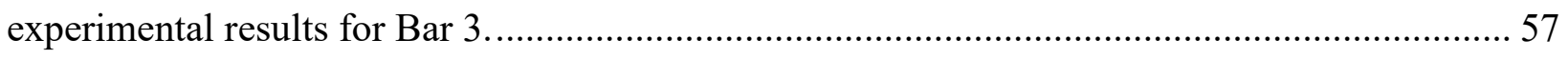

Figure 37: Raw experimental pressure data for a radial bar (Bar 4) (Rigby et al., 2015).......... 58

Figure 38: Comparison of FE model prediction for radial bar against maximum and minimum

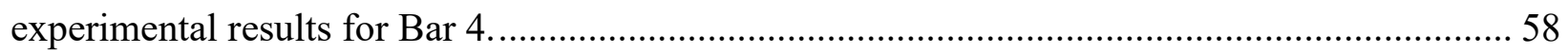

Figure 39: Comparison of analytical and experimental pressure-time history for the central bar (z

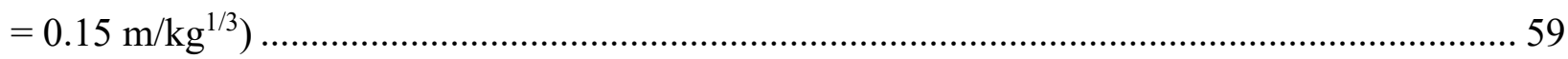

Figure 40: Comparison of analytical and experimental impulse-time history for the central bar (z

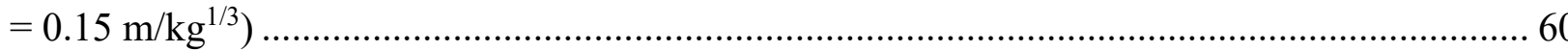

Figure 41: Comparison of analytical and experimental pressure-time history for the radial bar (z

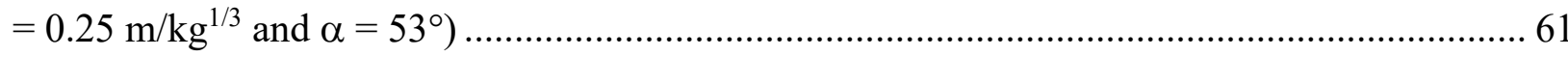

Figure 42: Comparison of analytical and experimental impulse-time history for the radial bar ( $\mathrm{z}=$

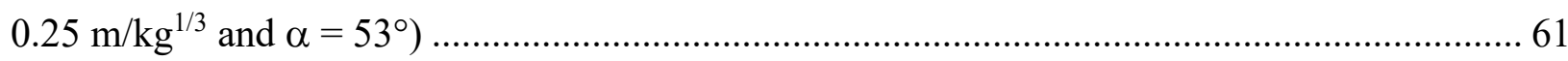


Figure 43: Time of arrival of the blast wave across the plate as predicted by the FE model.

Figure 44: Comparison of peak pressure variation across the plate as predicted by the FE model with experimentally reported values. 64

Figure 45: Comparison of impulse variation across the plate as predicted by the FE model with experimentally reported values.

Figure 46: Field-testing system used by Yang et al. (2019): (a) test setup and (b) instrumentation details (c) slab dimensions and reinforcement configuration. 66

Figure 47: (a) 2D model of Yang et al. (2019) (b) Propagation of blast pressures just prior to mapping.

Figure 48: (a) 3D model of Yang et al. (2019) (b) Propagation of blast pressures just after mapping (c) Support condition of the slab (simply supported). 67

Figure 49: Comparison of analytical and experimental reflected pressure-time histories for $5.6 \mathrm{~kg}$ ANFO with a $500 \mathrm{~mm}$ stand-off distance $\left(\mathrm{z}=0.282 \mathrm{~m} / \mathrm{kg}^{1 / 3}\right)$ 69 Figure 50: Comparison of analytical and experimental impulse-time histories for $5.6 \mathrm{~kg}$ ANFO with a $500 \mathrm{~mm}$ stand-off distance $\left(\mathrm{z}=0.282 \mathrm{~m} / \mathrm{kg}^{1 / 3}\right)$. 69

Figure 51: Mesh sensitivity analysis for modelling the air in 3D..................................... 71

Figure 52: Analysis time and total number of elements for various 3D air mesh sizes. ............ 72

Figure 53: Displacement-time history results for slab NC-3 ............................................. 73

Figure 54: Comparison of the deflected shapes and damage patterns for slab NC-3: (a) experimental results (Yang et al., 2019), (b) deflected shape predicted by the FE model, and (c) FE model deflected shape with plastic strain (damage) distribution. 75 Figure 55: Effective strain distribution in the blast-damaged slab on the (a) incident face and (b) distal face. .75 
Figure 56: Effective stress distribution in the slab at peak response on the (a) incident face and (b) distal face. 76

Figure 57: Axial strain distribution in steel reinforcement post-blast showing (a) yielded elements and (b) ruptured elements. 77

Figure 58: Field test setup of the RC slab under near-field explosion (Wang et al., 2012)......... 78

Figure 59: Geometry and reinforcement details of the RC slab (Wang et al., 2012)................ 78 Figure 60: (a) 3D model where TNT cylinder is detonated (b) Pressure distribution just prior to

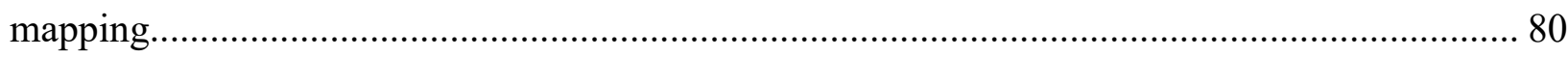

Figure 61: (a) 3D model where blast wave interacts with the slab (b) Mapping of pressures just prior to interaction (c) Support condition of the slab (fixed support). 80 Figure 62: Experimental damage to Slab E on the (a) incident face and (b) distal face (Wang et al., 2012)

Figure 63: Plastic strain (damage) distribution predicted by the FE model of Slab E on the (a) incident face and (b) distal face.

Figure 64: Effective stress distribution in the slab at peak response on the (a) incident face and (b) distal face 83

Figure 65: Effective strain distribution in the blast-damaged slab on the (a) incident face and (b) distal face. 83 Figure 66: Axial strain distribution in the steel reinforcement showing (a) dynamic yield strain and (b) maximum strains. 84 Figure 67: Plastic strain distribution on distal face of the slab with approximate spalling radius. 
Figure 68: Slab damage reported (a) in the experiment, (b) using LBE modelling method, (c) using CFD modelling method with spherical charge, and (d) using CFD modelling method with

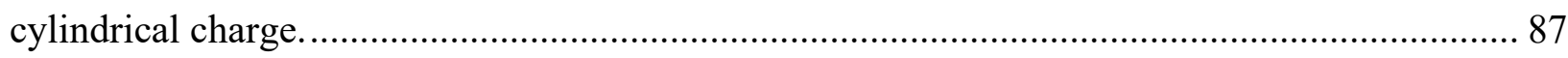

Figure 69: Field experimental setup for testing RC beams (Zhang et al., 2013)_........................ 91

Figure 70: Steel reinforcement detailing of beam B2-4 tested by Zhang et al. (2013)................. 92

Figure 71: (a) TNT cylinder is detonated in a 3D model (b) Pressure distribution just prior to

mapping

Figure 72: (a) Depiction of 3D model where blast wave interacts with the beam (b) Mapping of pressures just prior to interaction (c) Support condition of the beam (fixed support).................. 94

Figure 73: Comparison of the maximum experimental deflection with the predicted deflection by

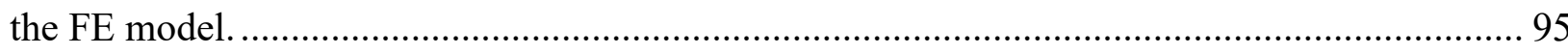

Figure 74: Damage to beam B2-4 (a) The overall destruction (b) Side face (c) Front face (d)

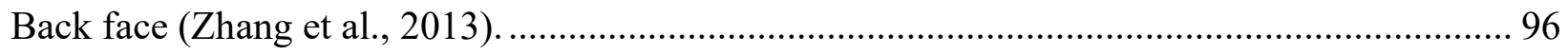

Figure 75: FE model prediction of the deflected shape of the blast-damaged beam.................... 96

Figure 76: Close-up of the damaged area in the FE model......................................................... 96

Figure 77: Effective strain distribution of the blast-damaged beam........................................... 98

Figure 78: Effective stress distribution of the beam at peak response......................................... 98

Figure 79: Axial strain distribution in steel reinforcement showing (a) dynamic yield strain and

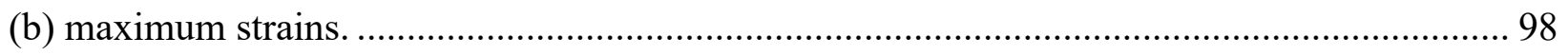

Figure 80: Effect of different support conditions on the displacement of the beam.................... 100

Figure 81: Deflected shape of blast-damaged beam using (a) fixed steel supports, (b) fixed nodal

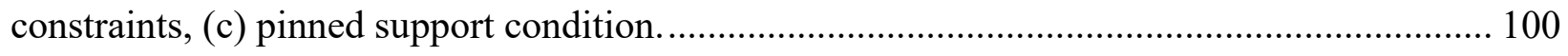

Figure 82: Beam deflections predicted by different FE models. .............................................. 101 
Figure 83: (a) Beam damage recorded in experiment (b) Beam damage computed by LBE (c) Beam damage computed by CFD with spherical charge (d) Beam damage computed by CFD with cylindrical charge. 102

Figure 84: (a) Cross-section details of reference column (b) Positioning of reference column relative to explosive charge. 105

Figure 85: Effect of transverse reinforcement spacing on lateral displacement. 107

Figure 86: Effect of transverse reinforcement spacing on length of spalling. 112

Figure 87: Section cut of columns for effective stress distributions. 115

Figure 88: Effect of transverse reinforcement spacing on residual axial capacity. 116

Figure 89: Effect of concrete cover on maximum and residual displacements. 119

Figure 90: Effect of concrete cover on length of spalling. 122

Figure 91: Effect of concrete cover on residual axial capacity. 125

Figure 92: Effect of axial load ratio on maximum and residual displacements. 128

Figure 93: Effect of axial load ratio on length of spalling. 133

Figure 94: Effect of axial load ratio on residual axial capacity. 133

Figure 95: P-M interaction diagram for the reference column. 135

Figure 96: Column shapes and cross-section detailing. (a) Square (b) Rectangular (bending about weak axis) (c) Rectangular (bending about strong axis) (d) Circular. 136

Figure 97: Effect of column shape on maximum and residual displacements. 139

Figure 98: Effect of columns shape on length of spalling. 139 


\section{Introduction}

Blast resistant design is a crucial consideration for critical infrastructure that is of high-risk and high-importance. Critical infrastructure refers to facilities, systems, and structures that are vital to national security, economic security, or public health and safety. These can include areas such as the national defence sector, energy industry, healthcare facilities, and many others. The inclusion of blast-resistance in design of critical infrastructure is becoming increasingly common as a result of terrorist attacks and accidental explosions. Historically, there have been many explosions that have caused severe structural damage as well as numerous casualties and fatalities. For instance, in Canada, there was the bombing of the Yugoslav Embassy in Ottawa in 1967, the bombing of the Cuban Embassy in Ottawa in 1974, and the bombing of Montreal Central Station in 1984. The most recent example was the Beirut port blast in 2020 that caused 204 deaths, 7500 injuries, and about $\$ 15$ billion in property damage leaving about 300000 people homeless.

In blast resistant design, performance of columns, which are primary load-bearing elements in structures is of particular importance. Columns must be designed in order to withstand damage directly sustained from blast loads. However, it is also critical to consider residual capacities of blast-damaged columns, as the failure of a key load-bearing member can result in the progressive and disproportionate collapse of an entire structure.

Explosions can be characterized as near-field, far-field, or contact depending on the size of the charge mass and stand-off distance to the structure. The Hopkinson-Cranz scaling law can be used to compare blasts of varying charge mass and stand-off distance (Baker et al., 1983). Blast waves which are produced at identical scaled distances will have identical peak overpressure, even though the charge mass and stand-off distance may be different. Other blast wave parameters, such as 
arrival time, positive phase duration, and impulse will differ. Scaled distance, denoted by $z$, is defined as follows:

$$
z=\frac{R_{1}}{W_{1}^{1 / 3}}=\frac{R_{2}}{W_{2}^{1 / 3}}
$$

where, $R$ is the range of the explosive and $W$ is the weight of the explosive.

Near-field explosions are typically considered to have a scaled distance of less than $1.18 \mathrm{~m} / \mathrm{kg}^{1 / 3}$ (ASCE, 2011). In this case, the pressure, impulse, and time of arrival of the blast wave varies along the incident face of the structure. As a result, the loading is complex and nonlinear as the blast wave propagates. Explosions are considered far-field when the scaled distance is greater than 1.18 $\mathrm{m} / \mathrm{kg}^{1 / 3}$. In this case, the loading on the structure can be assumed to be uniform and planar. Figure 1 shows the typical distribution of pressure, impulse, and arrival time for near- and far-field blast loading scenarios.

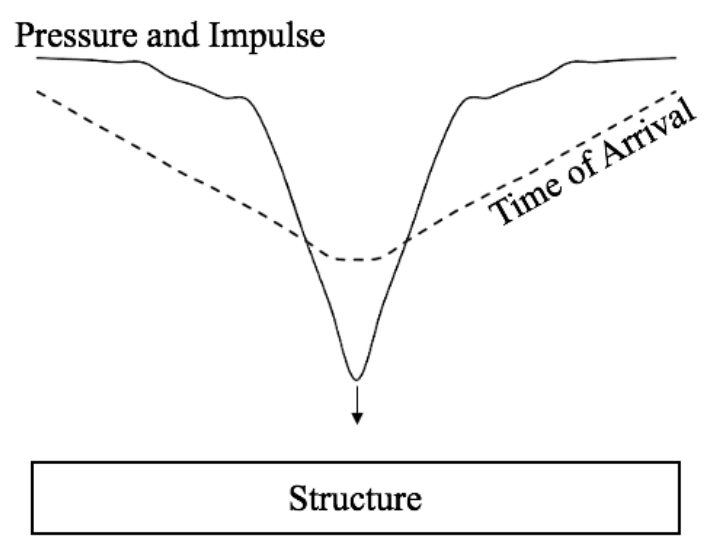

(a)

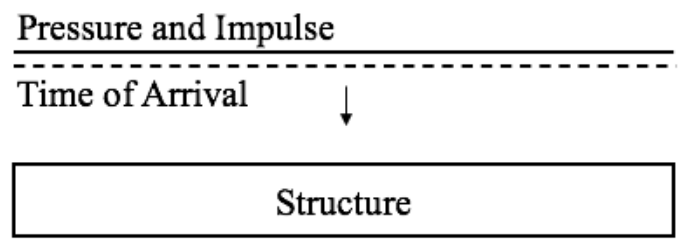

(b)

Figure 1: (a) Near-field and (b) far-field blast loading on a structure.

Failure modes of reinforced concrete (RC) columns under blast loading can be classified as either global or local (Zineddin \& Krauthammer, 2007). Global failure of RC columns under blast loading typically is a result of shear failure, flexural failure, or a combination of the two (Shi et al., 2008). Figure 2 depicts a schematic of deflected structural shapes for different global failure modes. Flexural failure is a ductile structural response initiated by yielding of longitudinal 
reinforcing steel followed by crushing of concrete at the compression zone. In the case of shear failure, a brittle failure occurs at low drift levels typically before the longitudinal reinforcing steel can reach strains beyond yielding. In this failure mode, the structure does not have sufficient time to develop any meaningful flexural response. Combined flexural-shear failure is a failure mode that falls between flexural and shear failure. It occurs as a result of shear distress, but after yielding of the longitudinal reinforcing steel has already occurred (Zhu et al., 2007). In addition to the structural details, the type of blast loading can also affect the global response of the column. Loading that has a very short duration in relation to the structure's natural frequency is referred to as impulsive loading. The response of the structure is sensitive to the impulse imparted by the blast load. This type of blast load with very short duration tends to induce shear damage in the structure. In comparison, when the blast load has a longer duration relative to the natural frequency of the structure, the loading is considered to be quasi-static and is sensitive to the peak pressure. This type of blast load tends to induce flexural damage in the structure (Shi et al., 2008).

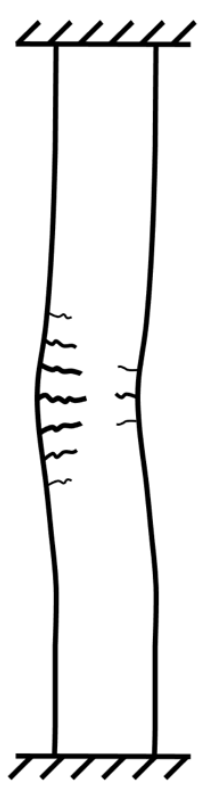

(a)

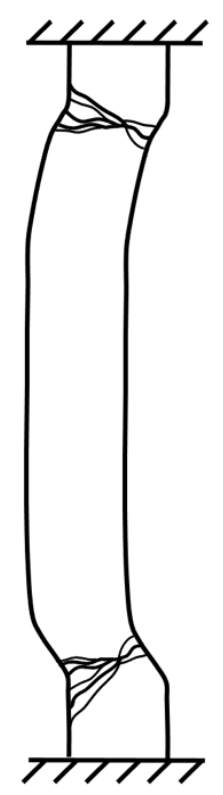

(b)

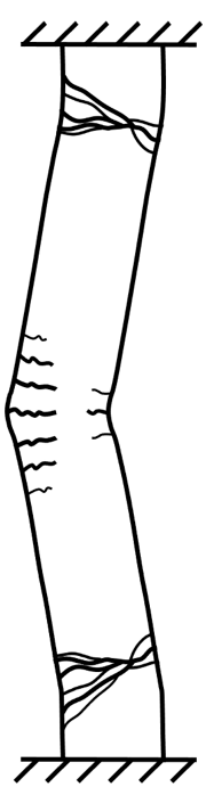

(c)

Figure 2: Global failure of RC columns under blast as a result of (a) flexural failure, (b) shear failure, and (c) flexural-shear failure. 
Local failure of RC columns mostly depends on concrete material properties, reinforcement detailing, and cross-sectional dimensions ( $\mathrm{Li} \& \mathrm{Hao}, 2014$ ). Local failure tends to be more significant and critical in near-field and contact blast loading scenarios in comparison to far-field blast loads. As demonstrated in Figure 3, localized failure can include concrete spalling, cratering, or complete breaching of the column. Concrete spalling is a result of the brittle tensile failure of the concrete material, while cratering occurs due to significant crushing as a result of the compressive failure of the concrete material.

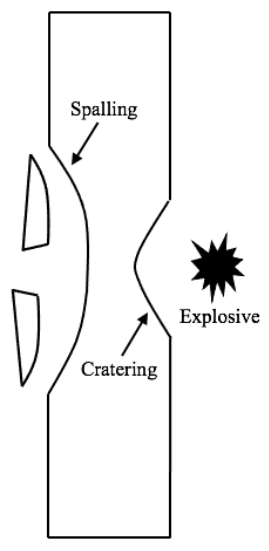

(a)

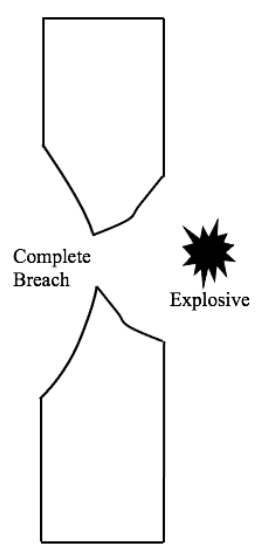

(b)

Figure 3: Local failure of RC columns under blast as a result of (a) cratering and spalling and (b) complete breach.

Finite element (FE) modelling can be used as a tool to predict structural response under blast loads and identify design parameters that can be used to improve blast resistance. Modelling of nearfield blast effects can be intricate as they cannot be assumed to be linearly applied. More importantly, it has been shown that the blast parameters predicted by the empirical relations of Kingery and Bulmash and the analytical equations used in ConWep are not accurate when the scaled distance is less than $0.4 \mathrm{~m} / \mathrm{kg}^{1 / 3}$ (Cormie et al., 2014; Dua et al., 2020).

The most common FE methods to model blast loads are either through the use of empirical/analytical equations or computational fluid dynamics (CFD). As the empirical/analytical equations have been reported to be inaccurate below $0.4 \mathrm{~m} / \mathrm{kg}^{1 / 3}$, CFD should be used to accurately 
model the explosive detonation phenomenon and its interaction with the structure in these instances (Trajkovski et al., 2014).

This study implements CFD for predicting blast load effects at scaled distances below $0.4 \mathrm{~m} / \mathrm{kg}^{1 / 3}$. The structural response of RC columns to these complex loadings is analyzed, and the influence of different design parameters is evaluated. A summary of the relevant analytical, experimental, and numerical studies available in the literature is provided in the following section. 


\section{Literature Review}

\subsection{Simplified Blast Models}

LS-DYNA offers a number of options to model blast loadings on structures. The LOAD_BLAST (LB) and LOAD_BLAST_ENHANCED (LBE) functions in LS-DYNA predict pressure loads from the detonation of conventional explosives based on a report by Randers-Pehrson and Bannister (1997). Randers-Pehrson and Bannister originally incorporated the Conventional Weapons (CONWEP) Effects program into DYNA2D and DYNA3D. CONWEP is an implementation of the empirical blast models of Kingery and Bulmash (Randers-Pehrson \& Bannister, 1997). These functions in LS-DYNA have limitations, such as their inability to model reflections (off of other structures/objects), confined explosions, shadowing effects from barriers, or different explosive charge shapes. However, for far-field blast loading, these functions have been proven to be accurate and efficient.

As a general rule, the use of empirical methods is considered inappropriate for prediction of blast loads in the near-field. The delineation between when it is appropriate to use simplified methods in favor of CFD in regard to a specific scaled distance is not explicitly defined. The selection of blast prediction method depends on the type of problem, size of problem (with regard to computational resources) and required accuracy. To elaborate, Low and Hao (2001) conducted a comprehensive investigation on the validity of different empirical equations and graphs for predicting blast loading from an explosion. It was found that different charts and empirical formulae available in the literature predict different blast loading parameters (specifically peak pressure, impulse, and duration), particularly when the scaled distance is less than $1 \mathrm{~m} / \mathrm{kg}^{1 / 3}$ or greater than $10 \mathrm{~m} / \mathrm{kg}^{1 / 3}$. These discrepancies between blast models tend to exist as a result of the complexities in blast testing, which make repeatability of test measurements difficult. In a 
numerical investigation by Shin (2014), it was concluded that the Friedlander equation is unable to capture overpressure history in the near-field due to expansion of detonation products, afterburning, and the formation of the Mach stem. The study also found that CFD simulations predicted peak overpressure, incident impulse, and normally reflected peak overpressure values much greater than those presented in UFC 3-340-02 when the scaled distance was less than 0.4 $\mathrm{m} / \mathrm{kg}^{1 / 3}$. Regardless, the author concluded that this was of little practical importance as CFD should be used in the near-field to estimate overpressure histories for design. Similarly, in an analysis of blast parameters in the near-field for spherical explosions, Karlos et al. (2016) concluded that the blast parameters proposed by Kingery and Bulmash should be used with caution at scaled distances below $0.4 \mathrm{~m} / \mathrm{kg}^{1 / 3}$. This inaccuracy arises as the Kingery and Bulmash charts were generated around measured and inferred data and were not verified in the near-field due to high pressures and temperatures. The authors also cited that the decay of peak overpressure in the near-field does not follow an exponential function as described by the Friedlander equation. However, as previously stated, the decision to use simplified blast analysis methods as opposed to CFD is not explicitly defined. Some researchers have found success in using simplified blast analysis methods to analyze blast loads on structures in the near-field, although typically at scaled distances above approximately $0.4 \mathrm{~m} / \mathrm{kg}^{1 / 3}$. These studies are considered in the following discussion.

Bao and $\mathrm{Li}$ (2010) used LS-DYNA with the built-in CONWEP blast model to analyze the residual strength of blast damaged RC columns. The model was verified using the laboratory results of RC columns subjected to loads generated by a blast simulator. The authors did not provide details regarding the experimental setup or properties of the test specimens. The columns were subjected to impulse loads of $0.77 \mathrm{psi}-\mathrm{s}, 1.76 \mathrm{psi}-\mathrm{s}, 1.9 \mathrm{psi}$-s, and $2.3 \mathrm{psi}$-s. The FE model was able to capture the residual deflection of the columns subjected to impulse loads of 0.77 psi-s and 2.3 psi-s with 
reasonable accuracy. However, it overestimated the residual deflections of the columns subjected to 1.76 psi-s and 1.9 psi-s impulse loads. No validation of column damage was presented in the study. The verified FE model was used to conduct a parametric analysis investigating the effects of transverse reinforcement ratio, axial load ratio, longitudinal reinforcement ratio, and column aspect ratio (depth-to-height ratio). The numerical simulations used a constant stand-off distance of $5 \mathrm{~m}$ with charge weights varying between 0 and 1 ton of trinitrotoluene (TNT). This range corresponded to a minimum scaled distance of $0.52 \mathrm{~m} / \mathrm{kg}^{1 / 3}$. The authors concluded that seismic detailing with respect to transverse reinforcement spacing significantly reduced the degree of direct blast-induced damage and subsequent collapse of the RC columns. They also observed that residual axial capacity increases with increasing longitudinal reinforcement ratio and decreasing aspect ratio. Based on the parameters analyzed, a formula was proposed capable of estimating the residual axial capacity of blast-damaged columns.

Li and Hao (2014) used the LOAD_BLAST function in LS-DYNA to study the effect of column dimensions and reinforcement mesh on the localized spall damage of RC columns under blast loads. Because of the limited number of reliable near-field blast tests on RC columns, the authors used the results of an RC slab test subjected to a blast at a scaled distance of $0.58 \mathrm{~m} / \mathrm{kg}^{1 / 3}$ to calibrate their numerical model. The FE model appeared to capture the damage profile quite well and was able to estimate the spall radius of the slab and residual deflection with good accuracy. The FE model was then used to investigate the influences of column dimensions and reinforcement mesh on the extent of concrete spalling in RC columns under near-field blast. Application of a FE model validated for slabs to evaluate the localized damage in RC columns is considered acceptable since under near-field blast the localized damage mostly depends on the material properties and reinforcement detailing than the type of structural member and end support conditions. Based on 
the analysis results, the authors found that spall damage was limited to a localized area and was insensitive to column stiffness and boundary conditions. However, they concluded that increasing column depth and number of reinforcing bars was effective in mitigating spall damage. The findings were then incorporated into a set of empirical relations to predict concrete spall damage based on explosion scenarios, column dimensions, and reinforcement conditions.

Li et al. (2017) used the LOAD_BLAST_ENHANCED function in LS-DYNA to examine the blast loading resistance of precast segmental RC columns. The numerical model was calibrated using the experimental results of an RC column subjected to $8 \mathrm{~kg}$ of TNT at a stand-off distance of $1.07 \mathrm{~m}$, for a scaled distance of $0.535 \mathrm{~m} / \mathrm{kg}^{1 / 3}$. The FE model was able to capture the deflectiontime history of the column with good accuracy. No validation of column damage was presented in the study. This model was then used to investigate the influence of shear keys and energy dissipation bars in segmental RC columns and how they compare with monolithic RC columns. The number of segments and level of post-tensioning in the RC columns was also examined. The columns were subjected to $5 \mathrm{~kg}$ of TNT detonated at mid-height at stand-off distances of $0.3,0.5$, and $1 \mathrm{~m}$, resulting in a scaled distance range of $0.18-0.58 \mathrm{~m} / \mathrm{kg}^{1 / 3}$. However, it should be noted that the FE model was only validated for a scaled distance of $0.535 \mathrm{~m} / \mathrm{kg}^{1 / 3}$. The results of the numerical simulations indicated that the segmental columns absorbed blast energy better than the monolithic columns and therefore reduced the amount of concrete spalling. The inclusion of energy dissipation bars had a limited effect on blast performance, while the inclusion of prestressing reduced central deflection of the columns.

$\mathrm{Li}$ and $\mathrm{Wu}(2018)$ employed the LOAD_BLAST function in LS-DYNA to analyze the behaviour of ultra-high performance concrete (UHPC) columns under blast loads. The authors calibrated the FE model using the results of two UHPC columns subjected to blast loads at scaled distances of 
0.46 and $0.70 \mathrm{~m} / \mathrm{kg}^{1 / 3}$. For the larger scaled distance $\left(0.70 \mathrm{~m} / \mathrm{kg}^{1 / 3}\right)$, the FE model appeared to capture the maximum deflection quite well, but slightly underestimated the residual deflection. For the smaller scaled distance $\left(0.46 \mathrm{~m} / \mathrm{kg}^{1 / 3}\right)$ the $\mathrm{FE}$ model underestimated the maximum deflection and overestimated residual deflection. The extent of damage and failure mode in RC columns were not evaluated in the verification study. The verified FE model was then used to derive pressure-impulse diagrams for UHPC columns of different concrete strength, column height, cross-section size, and reinforcement ratio. These diagrams can be used for evaluation of structural damage in UHPC columns. The authors concluded that increasing column size and concrete strength both resulted in an increase in the amount of pressure and impulse the UHPC column can sustain. Longitudinal reinforcement ratio increased the maximum pressure but had less of an influence on impulse. Finally, as column height increases, the amount of pressure and impulse the UHPC column can sustain decreases.

Rajkumar et al. (2020) used the LOAD_BLAST_ENHANCED function in LS-DYNA to investigate the effect of column geometry under various blast loads. The FE model was verified against field test results of a quarter-scale RC column subjected to a blast at a scaled distance of $0.535 \mathrm{~m} / \mathrm{kg}^{1 / 3}$. Details regarding the experimental setup or properties of the RC column were not provided. The FE model was able to capture the maximum deflection of the column quite well but appeared to underestimate the residual deflection. No validation of column damage was presented in the study. The validated FE model was used to conduct a parametric study to assess the influence of scaled distance, reinforcement ratio, and seismic detailing on the blast response of RC columns with different cross-sectional shapes. The numerical simulations used a range of scaled distances varying from 0.75 to $1.25 \mathrm{~m} / \mathrm{kg}^{1 / 3}$. The authors concluded that the circular column resulted in the lowest maximum and residual deflections in comparison to the square, hexagonal, and octagonal 
columns. They also noted that maximum deflections were reduced when reinforcement ratio was increased. It was also observed that seismic detailing had a minimal effect on the maximum displacements of the columns in comparison to conventional reinforcement detailing.

\subsection{Computational Fluid Dynamics Models}

With the advancement in computing technology, the application of CFD to analysis of concrete structures under blast loads has attracted significant interest as it eliminates the limitations of the aforementioned simplified analytical/empirical methods. By considering fluid-structure interactions and the complete detonation process, CFD provides a more accurate representation of the blast load and enables capturing important phenomena such as reflections, shadowing, and blast pressure clearing. Furthermore, it can model various explosive charge shapes which is an important consideration in analysis of structures under near-field blast loads.

Luccioni (2004) used CFD in the AUTODYN software to analyze the collapse of an entire RC structure as a result of blast loading. The structure considered for modelling was the Israel's Mutual Society of Argentina (AMIA) building. The building was subject to a terrorist attack and was used to verify the numerical model which provided insight into the structural collapse mechanism of the building. The FE model consisted of the RC structure (composed of the columns, beams and slabs) as well as the masonry walls. Due to the size of the model, some assumptions and idealization were made to reduce the analysis time. For instance, the mesh size of the structure was finer for the front of the building and lower levels, and was coarser in the back, as this area was not affected by the explosion. Furthermore, steel reinforcement was not explicitly modelled. Instead, an approximate material model was used to simulate the behaviour and response of the reinforced concrete. The authors also employed a $1 \mathrm{D}$ to $3 \mathrm{D}$ mapping procedure, with the assumption that the blast wave is spherically symmetrical until it encounters a rigid surface. The 
$400 \mathrm{~kg}$ TNT charge was modelled in a 1D environment and then mapped into a 3D model containing the structure. The run time of the analysis was approximately 310 hours. From the analysis results, the authors concluded that the structural collapse of the building was due to a gravitational mechanism produced by the failure of multiple load bearing columns on the lowerstoreys near the front of the building. While this type of analysis requires a great deal of computational resources, the authors suggested that it can be used for vulnerability assessment and selection of structural configurations that reduce the probability of progressive collapse in structures.

Baylot and Bevins (2007) tested the influence of exterior walls on the response of exterior columns using both numerical simulations and physical experiments. The structure analyzed was a twostory, two-bay portion of a building. The authors used quarter-scale experimental tests in conjunction with CFD modelling to plan a full-scale experiment. The quarter-scale tests and supporting numerical simulations were used to determine the charge standoff distance required to produce a desired level of column damage in the full-scale tests. The numerical simulations modelled the quarter-scale experiments, which were subjected to $7.1 \mathrm{~kg}$ of C4. The simulations were analyzed at stand-off distances ranging from 0.91 and $1.52 \mathrm{~m}$, resulting in scaled distances of approximately 0.45 and $0.74 \mathrm{~m} / \mathrm{kg}^{1 / 3}$. The blast loadings were initially modelled using the CTH software but were then extracted and applied to a column in DYNA3D to obtain the structural response. By using numerical modelling, the full-scale experiments could be planned to ensure failure of the exterior column, so that the potential for progressive collapse of the building could be evaluated. Based on the results of both the numerical and experimental investigations, it was found that while exterior walls fail very quickly under blast loads, they have a significant effect 
on the loads applied to the column and its response, as they delay the propagation of the blast through the structure and stop significant pressures from entering the inside of the structure.

Cui et al. (2015) used CFD in LS-DYNA to investigate the failure mechanism of RC columns under close-in blast loads. The authors first validated the model's ability to predict pressure in the near-field by modelling the experimental detonation of a cylindrical shaped Comp B charge, with the mass equivalent of $4 \mathrm{~kg}$ of TNT, at a stand-off distance of $1.295 \mathrm{~m}$, for a scaled distance of $0.82 \mathrm{~m} / \mathrm{kg}^{1 / 3}$. The FE model was able to capture the experimentally recorded pressure, while the empirical relations of the Kingery-Bulmash model underestimated the experimental pressure. To further verify the FE model, the authors also used the experimental results of an RC beam subjected to $0.75 \mathrm{~kg}$ TNT at a stand-off distance of $400 \mathrm{~mm}$, for a scaled distance of $0.44 \mathrm{~m} / \mathrm{kg}^{1 / 3}$. The FE model was able to capture the extent of crushing and spalling of concrete in the blast-damaged beam test specimen. Using this numerical model, a parametric analysis was conducted to investigate the influence of column dimensions and reinforcement ratio on the damage of RC columns. The columns were subjected to four levels of blast loads ranging in scaled distance from $0.11-0.2 \mathrm{~m} / \mathrm{kg}^{1 / 3}$. The results indicated that the damage under close-in blast loads was local such as spalling on the back and crushing on the front. It was also shown that larger column sections, with higher shear reinforcement ratios (lower stirrup spacings) and lower concrete cover can improve the performance under close-in explosions. Based on the findings, the authors developed a rapid damage assessment method for columns subjected to blast loads.

Codina et al. (2016) presented experimental results of two RC columns subjected to close-in blast loadings with no axial load. The fully-fixed columns, which used buried concrete blocks to provide fixity, were subjected to $8 \mathrm{~kg}$ of TNT detonated above the columns at stand-off distances of 60 and $100 \mathrm{~cm}$, for scaled distances of 0.3 and $0.5 \mathrm{~m} / \mathrm{kg}^{1 / 3}$ respectively. The results of the experiments 
were used to validate a CFD model in the ANSYS-AUTODYN software. The model was able to predict the residual displacements at three points along each column with good accuracy. The numerical model also provided good prediction of the post-blast damage and deflected shape of the columns. The validated model was used to propose a new set of parameters for a concrete constitutive model in AUTODYN to improve its accuracy for prediction of structural response. Thai and Kim (2018) used CFD in LS-DYNA to investigate damage of RC columns under blast loads. The ability of the FE model to simulate explosive pressure was validated using the experimental results of the detonation of $1 \mathrm{~kg}$ of equivalent TNT. Details regarding the stand-off distance to the pressure gauge was not provided. However, the comparison of the pressure-time histories reported in the experiment to those predicted by the FE model showed good agreement. The structural response computed by the FE model was validated using the experimental results of an RC column subjected to $8 \mathrm{~kg}$ of equivalent TNT at a stand-off distance of $600 \mathrm{~mm}$, for a scaled distance of $0.3 \mathrm{~m} / \mathrm{kg}^{1 / 3}$. The FE model was able to capture the maximum deflection of the column as well as the damage profile with good accuracy. Using the validate FE model, a parametric study was conducted on a series of six RC columns with different cross-section dimensions subjected to varying levels of blast loads with scaled distances ranging from 0.125 $0.295 \mathrm{~m} / \mathrm{kg}^{1 / 3}$. The analysis results showed that while stocky columns demonstrated only local damage, slender columns showed both local and global damage. Furthermore, columns subjected to explosions where the charge was placed at mid-height suffered significantly more damage compared to columns subjected to explosions where the charge was placed at the base. Finally, axial loading was found to increase the damage level of the slender columns but not the stocky columns. 


\subsection{Concluding Remarks}

A review of the above-mentioned studies shows that although there has been significant work conducted to assess the behaviour of RC structures under blast, there are still some important gaps that need to be addressed.

The majority of the parametric studies on RC columns have been conducted at scaled distances greater than $0.4 \mathrm{~m} / \mathrm{kg}^{1 / 3}$. These studies employed the simplified methods of blast load analysis by using empirical/analytical equations to predict blast loadings. Based on the findings of the studies discussed, it was generally found that higher reinforcement ratios for both longitudinal and transverse steel resulted in lower column deflections and increased residual axial capacity. Residual axial capacity also increased with decreasing column aspect ratio. Concrete spalling in columns was mitigated by increasing column depth and number of reinforcing bars.

As it has been reported that the empirical/analytical equations are inaccurate for scaled distances below $0.4 \mathrm{~m} / \mathrm{kg}^{1 / 3}$, CFD should be used to predict blast load effects on structures. The studies that employed CFD demonstrated that this modelling technique is capable of capturing both local and global response of RC columns with good accuracy. However, there is still a need to further investigate the influence of design parameters on the behaviour of RC columns under near-field blast loads with scaled distances less than $0.4 \mathrm{~m} / \mathrm{kg}^{1 / 3}$. For example, the effect of axial loading on the performance of RC columns under near-field blast loading has not been thoroughly investigated yet. Due to the absence of experimental data on RC columns with axial loading at scaled distances less than $0.4 \mathrm{~m} / \mathrm{kg}^{1 / 3}$, high-fidelity FE modelling is critical to investigate this parameter. Outside this scaled distance range, much of the test data available on RC columns tends to neglect the influence of axial force due to the complexities involved in applying and controlling the axial load during the test and potential damage to hydraulic loading equipment. 
Due to the complexity involved in near-field explosions, the number of studies conducted in this area is considerably less compared to studies on far-field blasts. Furthermore, experimentation costs associated with blast testing are high and field experiments can be difficult to conduct accurately. As a result, there is limited experimental data available that is reliable and accurate. FE modelling provides a cost-effective alternative to analyze performance of structures under blast loads. Therefore, development of reliable numerical models for accurate performance assessment of axially loaded columns under near-field blast loads is essential. These numerical models serve as a valuable tool to investigate the influence of different design parameters on the structural response of columns. The findings can lead to the development of more efficient and reliable design methodologies. 


\section{Objectives}

In the current state of the literature, the influence of RC column design parameters under blast loads has largely been analyzed using simplified methods to simulate the loading effects. These simplified methods use analytical/empirical equations to compute blast loads, which are not accurate in determining blast wave parameters for scaled distances less than $0.4 \mathrm{~m} / \mathrm{kg}^{1 / 3}$. Therefore, for these cases, the CFD modelling methodology would provide better prediction as it has the ability to model the detonation process of the explosion, propagation of the blast wave, and its interaction with the structure.

The main objective of this research is to investigate the effects of various design parameters on the performance of RC columns under blast loading with scaled distance less than $0.4 \mathrm{~m} / \mathrm{kg}^{1 / 3}$. In order to accomplish this, the CFD model's ability to capture blast wave parameters for scaled distances less than $0.4 \mathrm{~m} / \mathrm{kg}^{1 / 3}$ (such as pressure and impulse) is verified. Additionally, the ability of the CFD model to compute the local and global response of structural members under near-field explosions is demonstrated by analysis of various RC test specimens. Finally, through a parametric study, the influence of transverse reinforcement spacing, concrete cover, axial load ratio, and column shape on the performance of RC columns under near-field blast loads is evaluated. RC column performance is measured using maximum and residual lateral displacements, length of damage zone, stress conditions in concrete and reinforcement, and residual axial capacity. 


\section{Modelling Methodology}

In this section, the FE modelling methodology used in LS-DYNA for the verification and parametric studies described in Sections 0 and 1 is discussed in two parts: 1) structural modelling and 2) air and explosive modelling. LS-DYNA has the option to use implicit or explicit time integration to solve the FE problem. The explicit analysis utilizes the central difference method to solve the equation of motion and was selected for this research as it tends to be less computationally intensive than the implicit method. In contrast, the implicit solver has difficulties converging the solution when there is a high degree of material nonlinearity, which is often the case when modelling structures under blast loading.

\subsection{Structural Modelling}

\subsubsection{Concrete}

Concrete was modelled using eight-node hexahedron solid finite elements. A constant stress solid element formulation was used as these elements provide an efficient method of analysis and are acceptably accurate for even severe deformations (Hallquist, 2006), which is critical in modelling the response of structures under blast loads.

\subsubsection{Material Model}

LS-DYNA provides several concrete constitutive models. Some of the most commonly used include material type material type 084 (MAT_WINFRITH_CONCRETE), material type 072R3 (MAT_CONCRETE_DAMAGE_REL3), and material type 159 (MAT_CSCM). The performance of each of these concrete material models was evaluated under near-field explosions and one was selected for use in this research.

MAT_WINFRITH_CONCRETE is a smeared (or pseudo) crack model developed by Broadhouse and Neilson (1987). This model was considered because of its unique ability to model crack 
progression in concrete. Figure 4 shows the computed damage profile of an RC slab tested by Wang et al. (2012), which was one of the structural members used to validate the FE model in this research. The slab specimen was subjected to $0.64 \mathrm{~kg}$ of TNT detonated at a distance of $500 \mathrm{~mm}$ from the surface of the slab, giving a scaled distance of $0.591 \mathrm{~m} / \mathrm{kg}^{1 / 3}$. The dimensions of the slab were $1250 \times 1250 \times 50 \mathrm{~mm}$. It was reinforced in both directions with $6 \mathrm{~mm}$ diameter steel bars and was fully fixed at the ends. For more detailed information regarding the experimental setup and properties of the RC slab, refer to Section 5.2.2.1. The damage profile presented in Figure $4 \mathrm{~b}$ was predicted using MAT_WINFRITH_CONCRETE. The blue areas indicate uncracked sections while the red areas indicate fully cracked sections. The black lines extending from the centre of the slab indicate the crack pattern in the concrete. While investigating different material models, it was clear that MAT_WINFRITH_CONCRETE was good at predicting local damage and crack propagation. However, the inclusion of strain rate effects in this concrete model can lead to energy losses in the system (LSTC, 2017a). As a result of these energy losses, maintaining numerical stability proved difficult when strain rate effects were included, often resulting in premature termination of the analysis due to convergence problems. However, strain rate effects are a crucial consideration in the analysis of blast loads on structures, and therefore this material model was discarded due to this limitation. 
$\underline{\text { Incident Face }}$

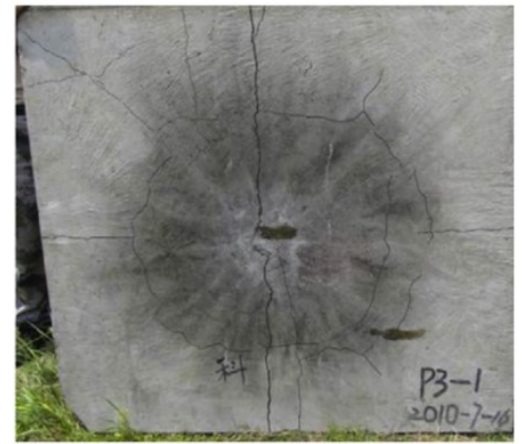

(a)
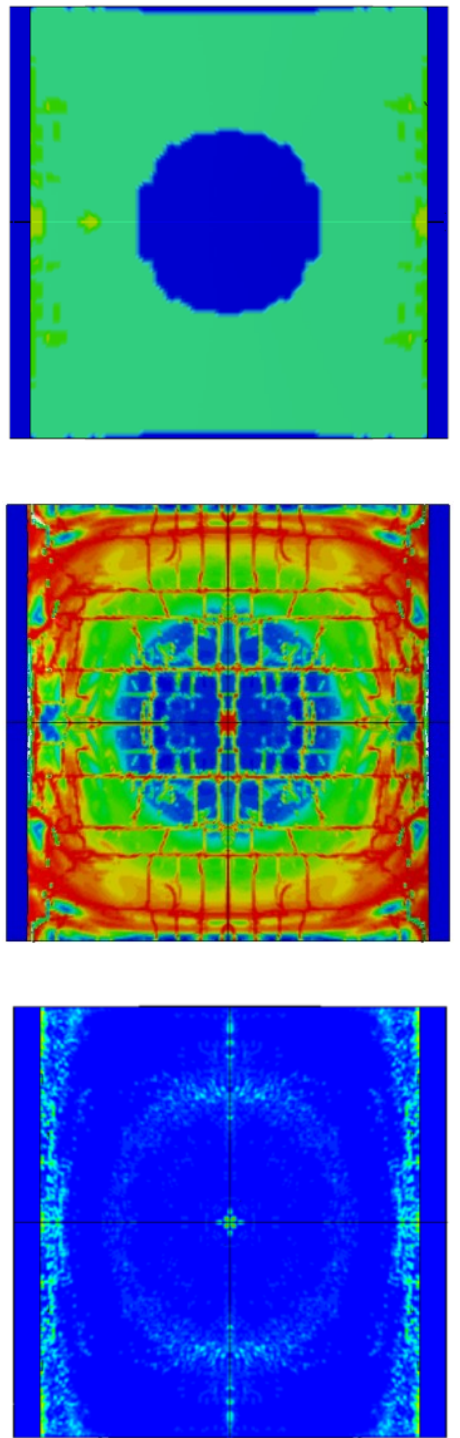

(b)

(c)
Distal Face
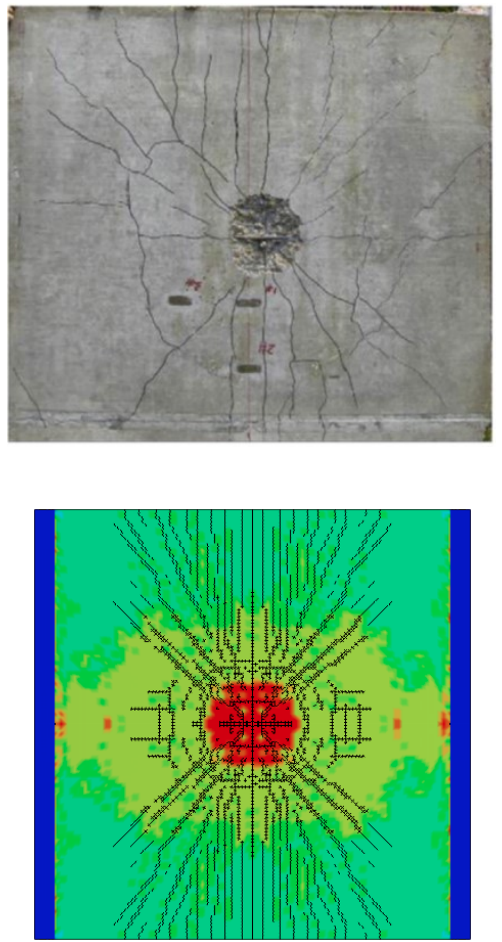

History Variable\#1

$3.000 \mathrm{e}+00$

$2.700 \mathrm{e}+00$

$2.400 \mathrm{e}+00$

$2.100 \mathrm{e}+00$

$1.800 \mathrm{e}+00$

$1.500 \mathrm{e}+00$

$1.200 \mathrm{e}+00$

$9.000 \mathrm{e}-01$

$6.000 \mathrm{e}-01$

$3.000 \mathrm{e}-01$

$0.000 \mathrm{e}+00$

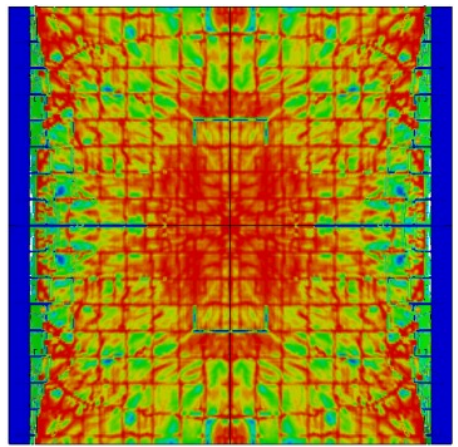

Effective Plastic Strain

$2.000 \mathrm{e}+00$

$1.800 \mathrm{e}+00$

$1.600 \mathrm{e}+00$

$1.400 \mathrm{e}+00$

$1.200 \mathrm{e}+00$

$1.000 \mathrm{e}+00$

$8.000 \mathrm{e}-01$

$6.000 \mathrm{e}-01$

$4.000 \mathrm{e}-01$

$2.000 \mathrm{e}-01$

$0.000 \mathrm{e}+00$

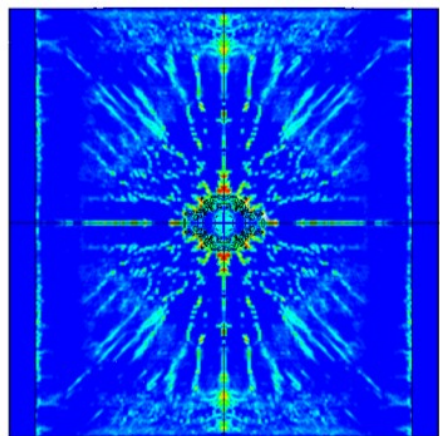

Plastic Strain

$9.990 \mathrm{e}-01$

8.991e-01

7.992e-01

$6.993 \mathrm{e}-01$

$5.994 \mathrm{e}-01$

4.995e-01

$3.996 \mathrm{e}-01$

2.997e-01

$1.998 \mathrm{e}-01$

$9.990 \mathrm{e}-02$

$0.000 \mathrm{e}+00$

(d)

Figure 4: Damage profile of RC slab tested by Wang et al. (2012) obtained from: (a) experiment,

(b) Winfrith concrete model, (c) KCC concrete model, and (d) CSCM concrete model. The incident face is shown in the left and the distal face on the right. 
MAT_CONCRETE_DAMAGE_REL3 (also referred to as KCC) is a three-surface plasticitybased model developed by Karagozian and Case (1994) that includes damage and strain rate effects. It is commonly used in the literature as it requires little effort from the user to define the model. The model can generate material properties of concrete based on a single input parameter, the unconfined compressive strength of concrete. The RC slab presented in Figure 4a was also modelled using MAT_CONCRETE_DAMAGE_REL3, for which the damaged profile is presented in Figure 4c. In this model, the blue areas indicate completely undamaged elements while the red areas indicate fully damaged elements. It is immediately clear that the concrete material model has a significant effect on the damage profile of the slab. While validating the FE model used in this research, this material model predicted the damage profiles of structural members quite well. However, the material model tended to interact poorly with support structures (usually steel members). Often an unrealistic degree of erosion of concrete elements occurred in the area in contact with the supports. Erosion for this material model must be defined using MAT_ADD_EROSION, which is a separate keyword and is highly mesh dependent. As the erosion changed significantly with loading and mesh size, this led to inaccurate predictions of displacements. The excessive deletion of elements also led to numerical instabilities and premature termination of analyses. Based on these limitations, this material model was not used in the research.

MAT_CSCM was developed by the U.S. Department of Transportation in conjunction with APTEK based on fourteen years of defense research contracts (Murray, 2007). It has the option to specify default material properties for normal strength concrete using the MAT_CSCM_CONCRETE option. While the other concrete material models were explored, CSCM proved to be the most consistent and reliable in terms of its numerical stability and ability 
to accurately predict displacements when validating the FE model. It also proved to be a good predictor of local damage in concrete without excessively or unrealistically eroding elements (see Figure 4d). As a result, this material model was selected for use in this research study.

A number of other researchers have also found success in using CSCM to model concrete under a variety of different loading conditions. For instance, in a review of concrete constitutive models, Cui et al. (2017) found that both KCC and CSCM led to reasonable predictions of structural response for concrete structures subjected to blast loads. They also found that these material models most accurately predicted concrete crater diameter induced by a steel projectile. Castedo et al. (2021) tested three RC slabs under close-in blast loads, one of which were used to investigate the performance of different concrete constitutive models in LS-DYNA. CSCM was able to provide the best prediction for the acceleration values of the slab, which were collected and reported in the experiment using accelerometers. It also showed good results when comparing slab damage and was able to predict the tensile cracking pattern on the distal face of the slab, whereas KCC overestimated the damage on both the incident and distal faces of the slab. Fan and Yuan (2014) conducted numerical simulations of low-velocity impact tests on RC beams using the CSCM, Winfrith, and KCC models. The experimental results from a drop hammer impact test were used for the investigation. The results showed that the peak hammer-beam contact forces were overpredicted by the Winfrith model, while both average and peak values from the CSCM and KCC models agreed well with experimental results. However, the damage distributions of the RC beams showed better agreement when CSCM was used. Therefore, the authors adopted CSCM for use in further simulations. Kim et al. (2015) used different concrete material models in LSDYNA to analyze projectile penetration with a concrete target, for which CSCM showed the best agreement with experimentally reported penetration values. A number of researchers have also 
analyzed the capability of LS-DYNA concrete models in relation to seismic applications. While seismic analysis is not the focus in this research, the following studies support CSCM's ability to model a wide variety of loading scenarios. In an evaluation of the performance of LS-DYNA concrete models for seismic applications, Coleman (2016) found that both the CSCM and Winfrith material models demonstrated all the necessary capabilities for use in seismic applications. However, the KCC model was not recommended for use in seismic applications due to damage accumulation limitations. Furthermore, inconsistencies were found when strain rate effects were included in KCC. It was observed that KCC exceeded manually specified dynamic increase factors (DIF) at high strain rates and showed instability in the results for low strain rates. Bohara et al. (2018) concluded similar results in a review of the use of concrete constitutive models in LSDYNA for analysis of RC structures under reversed cyclic loading. It was found that CSCM, Winfrith, and KCC were all able to capture the cyclic load reversal behaviour, but CSCM was the only model that captured softening behaviour with good accuracy and was able to accurately predict the force-displacement response and damage pattern. The study concluded that CSCM should be used for lateral cyclic loading simulations. The results of these studies provide confidence in CSCM's ability to model concrete response under a variety of loading conditions including blast loads. In the following, the concept behind CSCM and some of its relevant features are briefly discussed.

MAT_CSCM is a smooth or continuous surface cap model. Cap models use a classical plasticity approach, wherein the yield condition of the material is the defining characteristic. The yield condition limits the degree of stress supported during elastic deformation. Once this condition is reached, the material can continue to deform in an inelastic manner. This type of constitutive model (cap model) was developed in order to predict the effect of conventional weapons on the response 
of structures (Sandler, 2005). Figure 5, shows the yield surface, which is composed of a shear failure surface and a moveable hardening cap connected by a smooth and continuous transition.

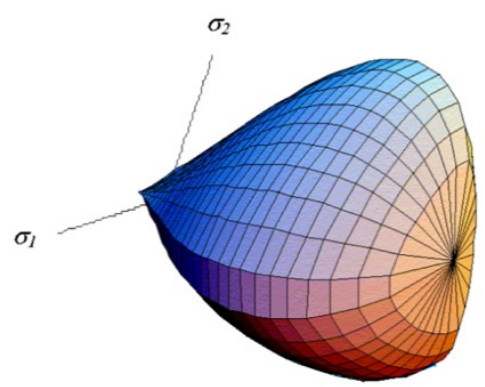

(a)

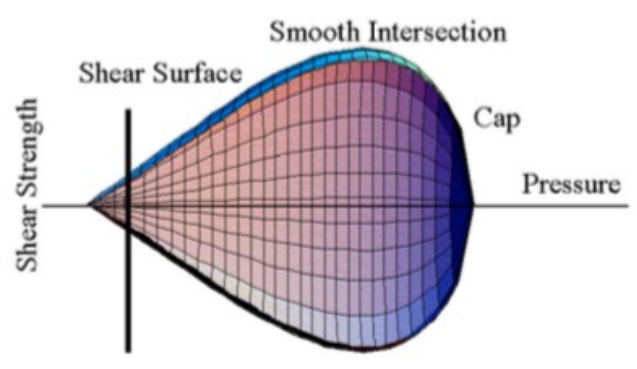

(b)

Figure 5: CSCM yield surface in (a) three dimensions and (b) two dimensions (Murray, 2007).

For concrete in tension region and in compression region without confinement, the concrete strength is modelled by the shear failure surface, shown in Figure 5. Confinement of concrete is considered in this model through a combination of the hardening cap and shear surfaces. The cap models plastic volume changes by expanding and moving outwards to model compaction, and contracting and moving inwards to model expansion (or dilation). The post-peak behaviour of concrete is also considered in CSCM. The strength reduction (softening behaviour) of concrete is modelled using a damage formulation, which is based on either brittle or ductile damage depending on whether the concrete is subject to tensile or compressive lateral stresses. The post-peak response takes into account both strain-softening due to strength degradation and modulus reduction as it relates to a decrease in loading/unloading slopes. CSCM also has the option to include strain rate effects which scale the concrete strength in tension and compression. The developer of this model cited that the CEB specifications for DIF are not a good fit for concrete tensile data, and therefore the DIF used in CSCM are based on their experimental work on defense contracts. The DIF used in CSCM, which are shown in Figure 6, provide a good fit for both tension and compression data for concrete (Murray, 2007). 


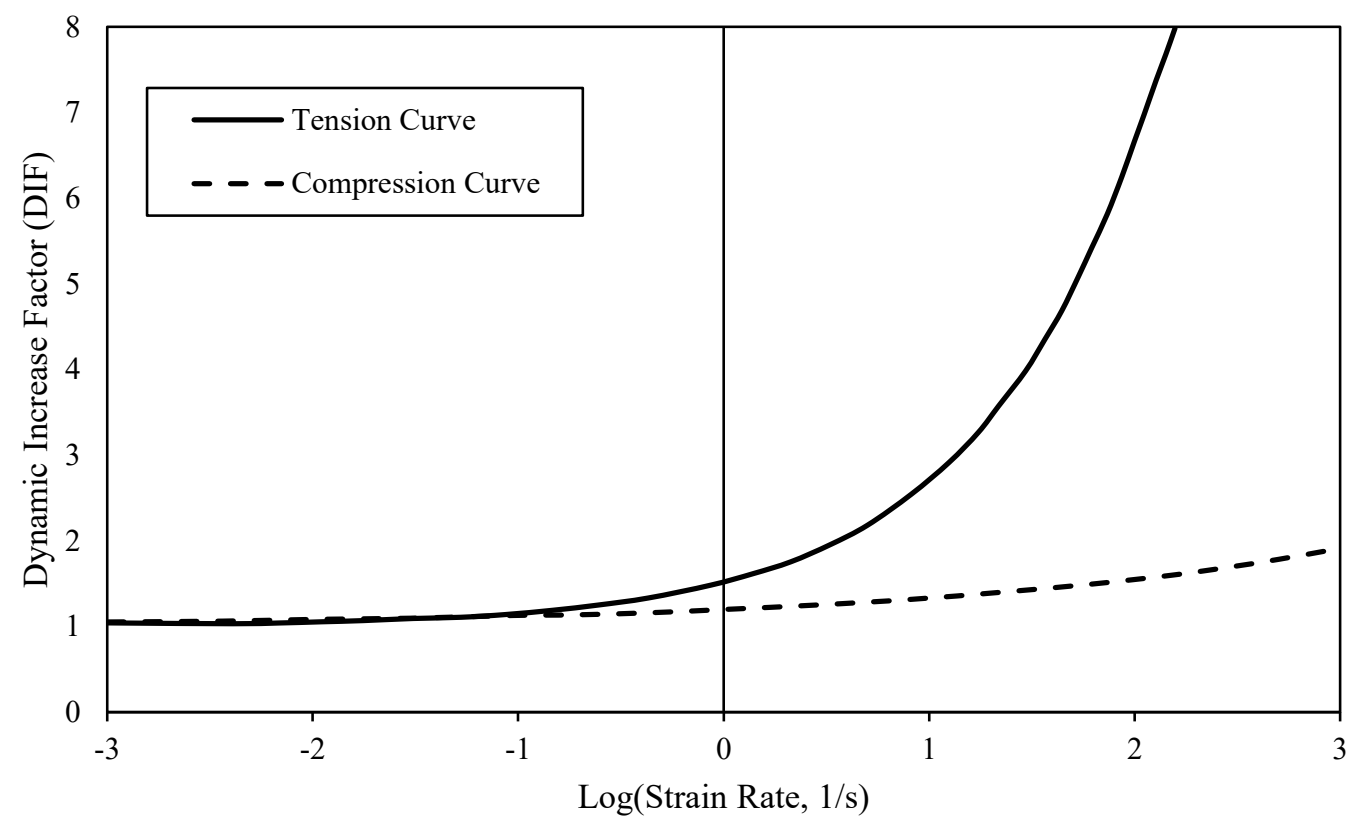

Figure 6: DIF in tension and compression for CSCM concrete (Murray, 2007).

Figure 7 presents the uniaxial compressive and tensile stress-strain responses computed by the CSCM model for a single plain concrete element with a cylindrical compressive strength of 30 MPa. The stress-strain graph shows both the elastic and inelastic response of the concrete. The unconfined compressive strength of the concrete is approximately ten times the unconfined tensile strength, which is in agreement with typical concrete test data. The results of a triaxial compression test also computed using a single element simulation are shown in Figure 8. The results demonstrate the material model's ability to handle strength increase due to confining pressures. 


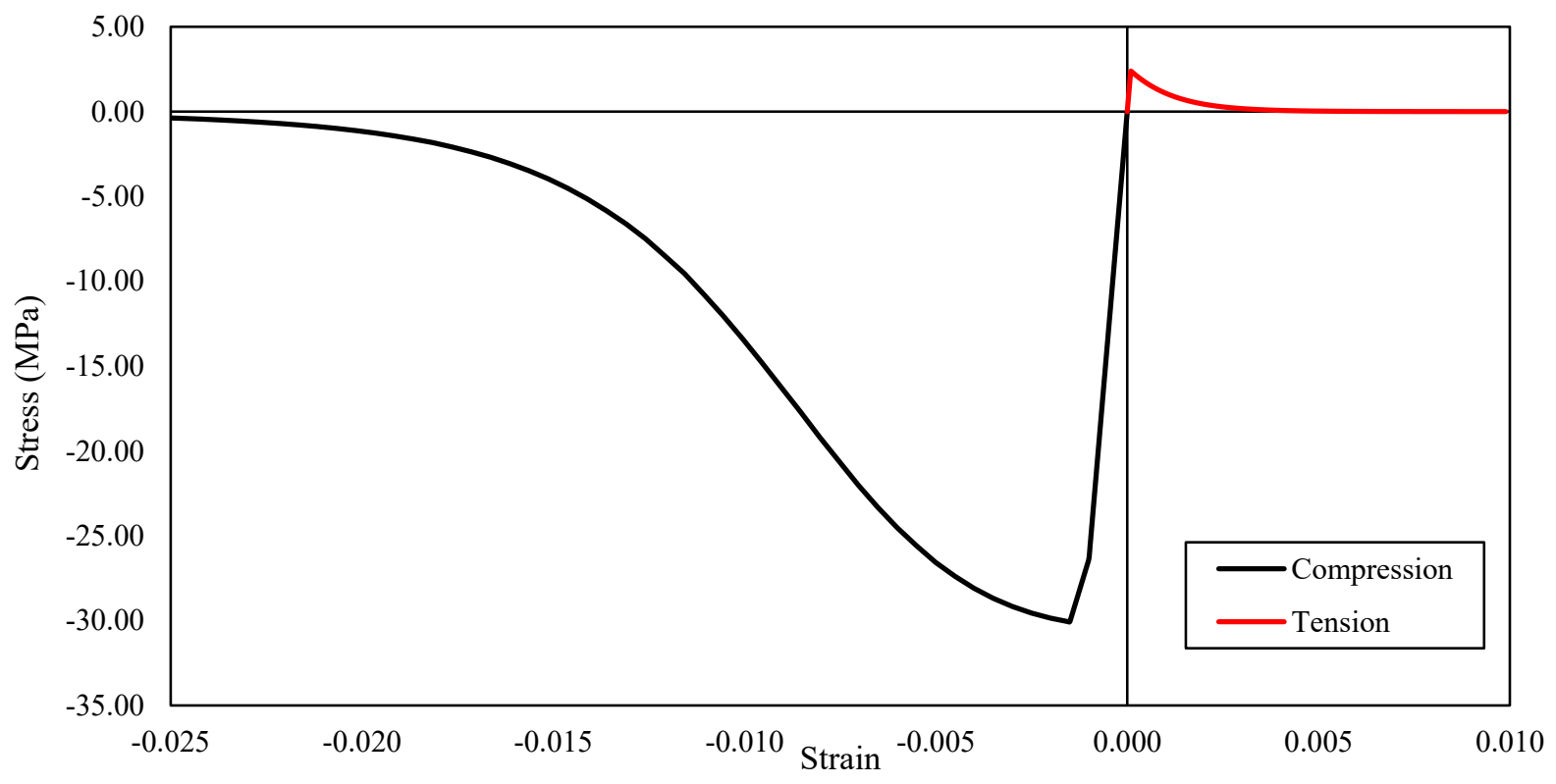

Figure 7: Uniaxial stress-strain curves of concrete with unconfined compressive strength of 30 MPa computed by CSCM (Murray, 2007).

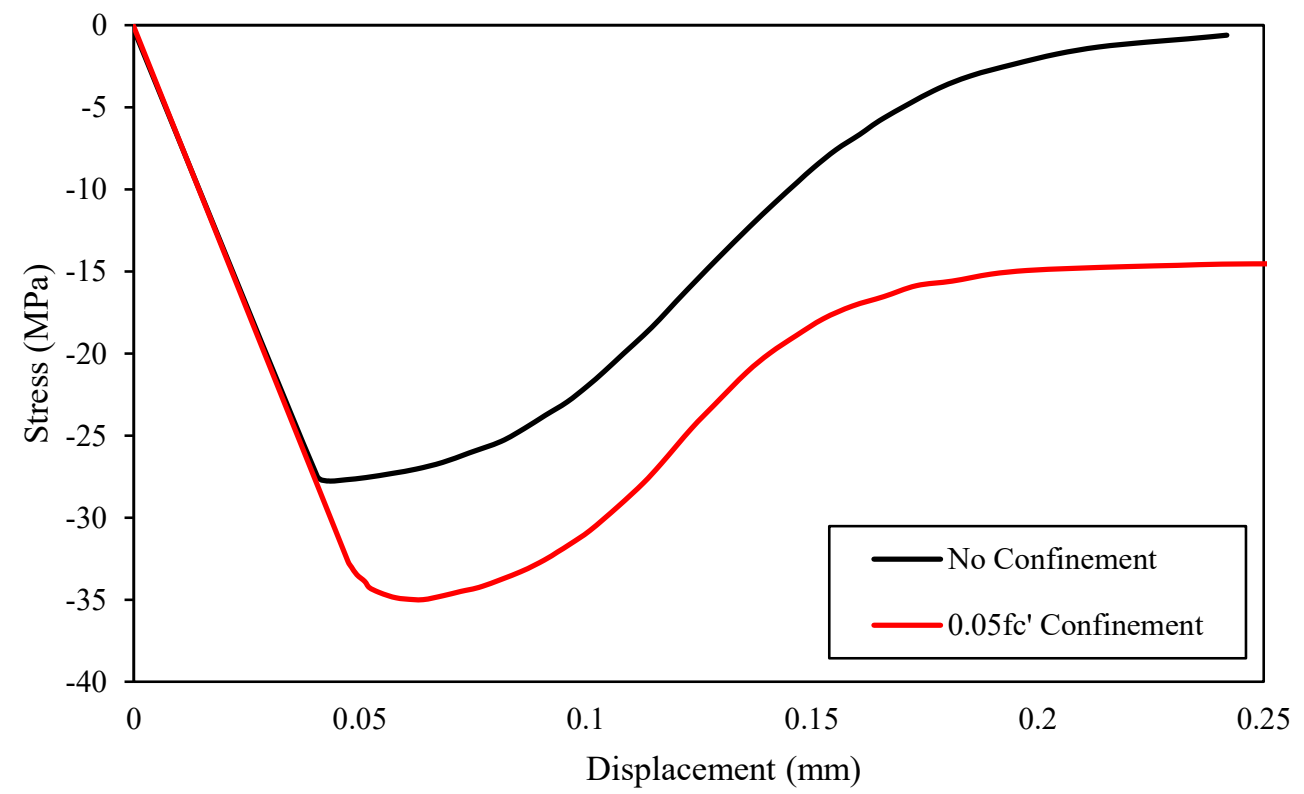

Figure 8: Results of a triaxial compression test on concrete using CSCM (Murray, 2004).

Murray (2004) also provided an extensive validation of CSCM on its ability to simulate the response of concrete to a variety of loading conditions. In order to evaluate CSCM's ability to model damage in plain concrete, the material model was used to simulate concrete cylinder and beam tests. CSCM was able to simulate the typical damage modes observed in concrete cylinder 
tests, namely splitting in tension and diagonal failure in compression. CSCM was also able to model the damage mode of a plain concrete beam under dynamic impact with good accuracy. The investigation also analyzed the model's ability to simulate deformation and damage of an underreinforced and an over-reinforced concrete beam subject to drop tower impact tests. The model's ability to simulate a bogie vehicle impact test on an RC beam was also evaluated. CSCM was able to model the damage and deflection-time history of the beams with good accuracy.

In summary, while other material models were explored for use in this research, CSCM consistently provided good predictions of both global and local structural response. This conclusion was found to be consistent with the findings of other research studies. Furthermore, CSCM has been validated against several experiments and has proved a good predictor of concrete response to a variety of loading conditions. As a result, CSCM was used for all numerical simulations in this research.

\subsubsection{Mesh Sensitivity Analysis}

A mesh sensitivity analysis for concrete solid elements was conducted by applying a displacementcontrolled axial load to an RC column. The displacement was applied at a rate of $0.01 \mathrm{~mm} / \mathrm{ms}$. The column details are provided in Figure 9 and the material properties of the concrete and steel are provided in Table 1. The base of the column was fully fixed from translation and rotation in all directions. The top of the column was also constrained from translation in the $\mathrm{x}$ and $\mathrm{y}$ directions and rotation in all directions. However, it was free to translate in the z direction in order to apply the axial loading. Figure 10 shows the RC column mesh for various sizes of solid concrete elements. The analysis results presented throughout this research were conducted on an Intel Core i7-4790 desktop with four CPU cores at $3.60 \mathrm{GHz}$. 

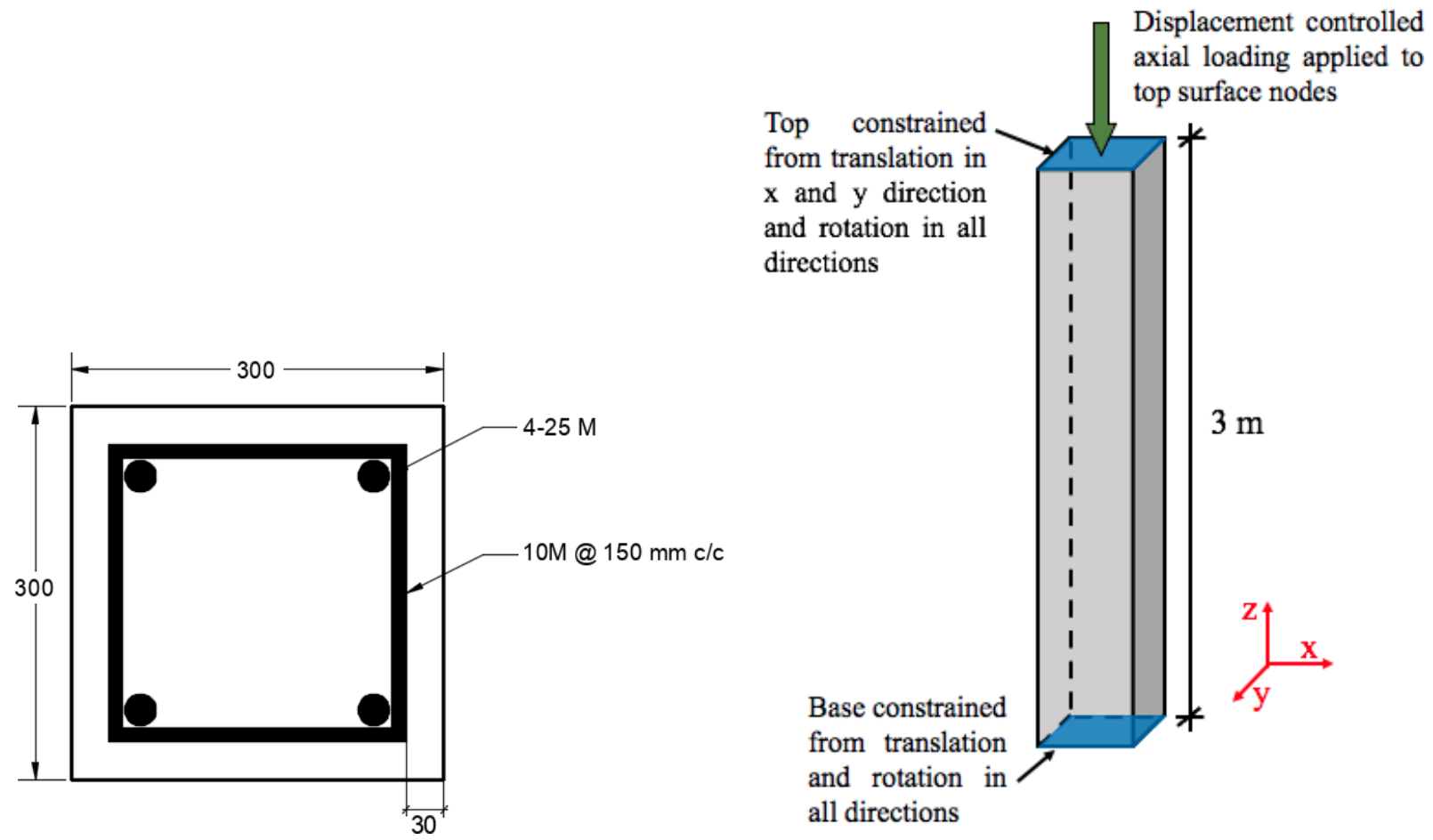

Figure 9: Detailing of RC column used in concrete mesh sensitivity analysis.

Table 1: Concrete mesh sensitivity analysis material properties.

Unconfined compressive strength of concrete: $\quad f_{c}{ }^{\prime}=40 \mathrm{MPa}$

Yield strength of reinforcing steel: $\quad f_{y}=400 \mathrm{MPa}$

Ultimate strength of reinforcing steel: $\quad f_{u}=600 \mathrm{MPa}$

Young's Modulus: $\quad E=200 \mathrm{GPa}$ 


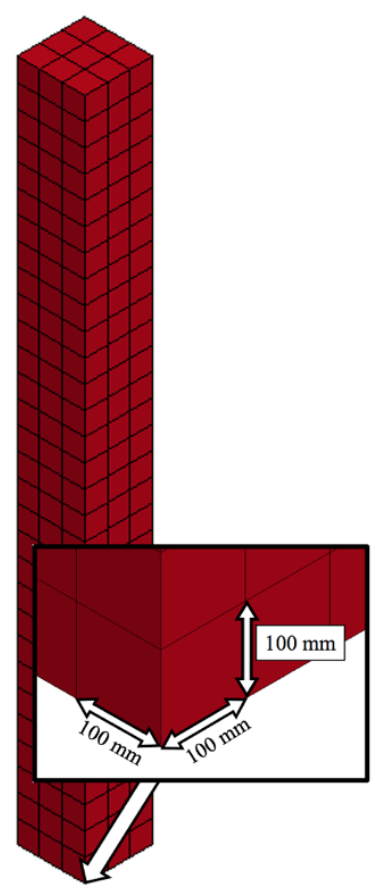

(a)

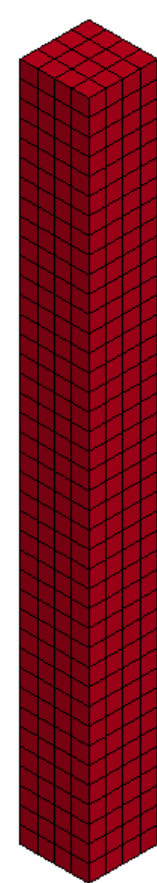

(b)

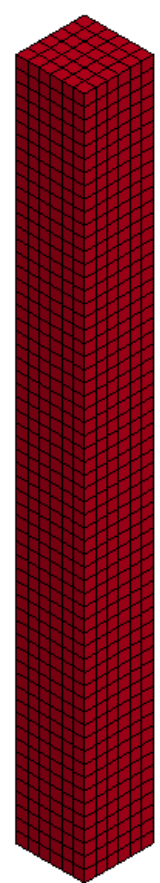

(c)

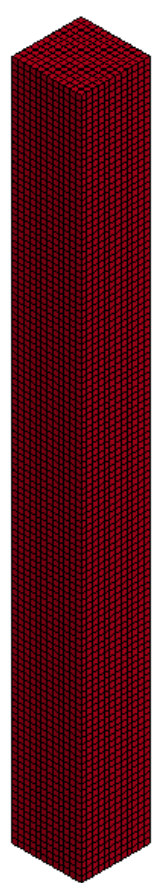

(d)

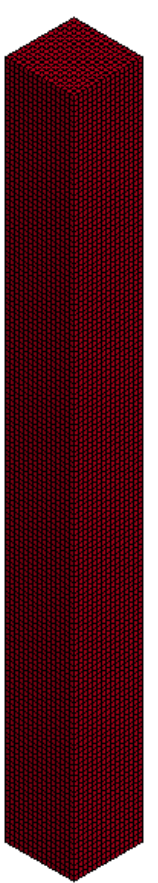

(e)

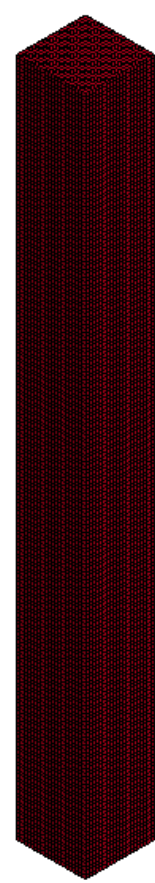

(f)

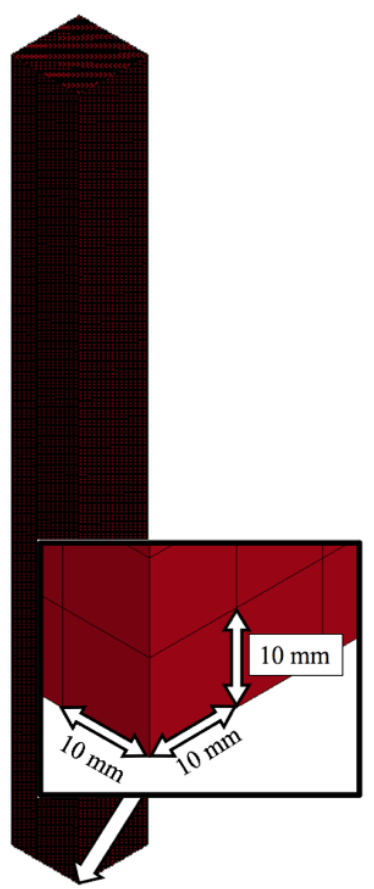

(g)

Figure 10: Mesh sizes of solid concrete elements for the RC column used in the sensitivity analysis: (a) $100 \mathrm{~mm}$ (b) $75 \mathrm{~mm}$ (c) $50 \mathrm{~mm}$ (d) $25 \mathrm{~mm}$ (e) $20 \mathrm{~mm}$ (f) $15 \mathrm{~mm} \mathrm{(g)} 10 \mathrm{~mm}$.

Figure 11 shows the load-displacement results obtained from the mesh sensitivity analyses. The theoretical axial capacity, which includes the increase in concrete strength due to confinement effects computed based on the work of Mander et al. (1988), is also plotted for reference. It can be seen that the analysis results converge at a mesh size of $15 \mathrm{~mm}$. Further reducing the mesh size has little effect on the force-displacement response but increases both analysis time and number of concrete elements significantly, as listed in Table 2. Figure 12 shows the graphical relationship between mesh size of concrete, analysis time, and total number of elements. The analysis time and total number of concrete elements sharply increases when decreasing the mesh size from $15 \mathrm{~mm}$ to $10 \mathrm{~mm}$. However, for this research concrete mesh sizes were typically selected in the range of $5-10 \mathrm{~mm}$ as it provided a more detailed damage profile, especially in regard to heavily cracked regions. This was particularly important when modelling slabs, as it provided more elements through their thickness enabling a more accurate calculation of nonlinearity effects. 


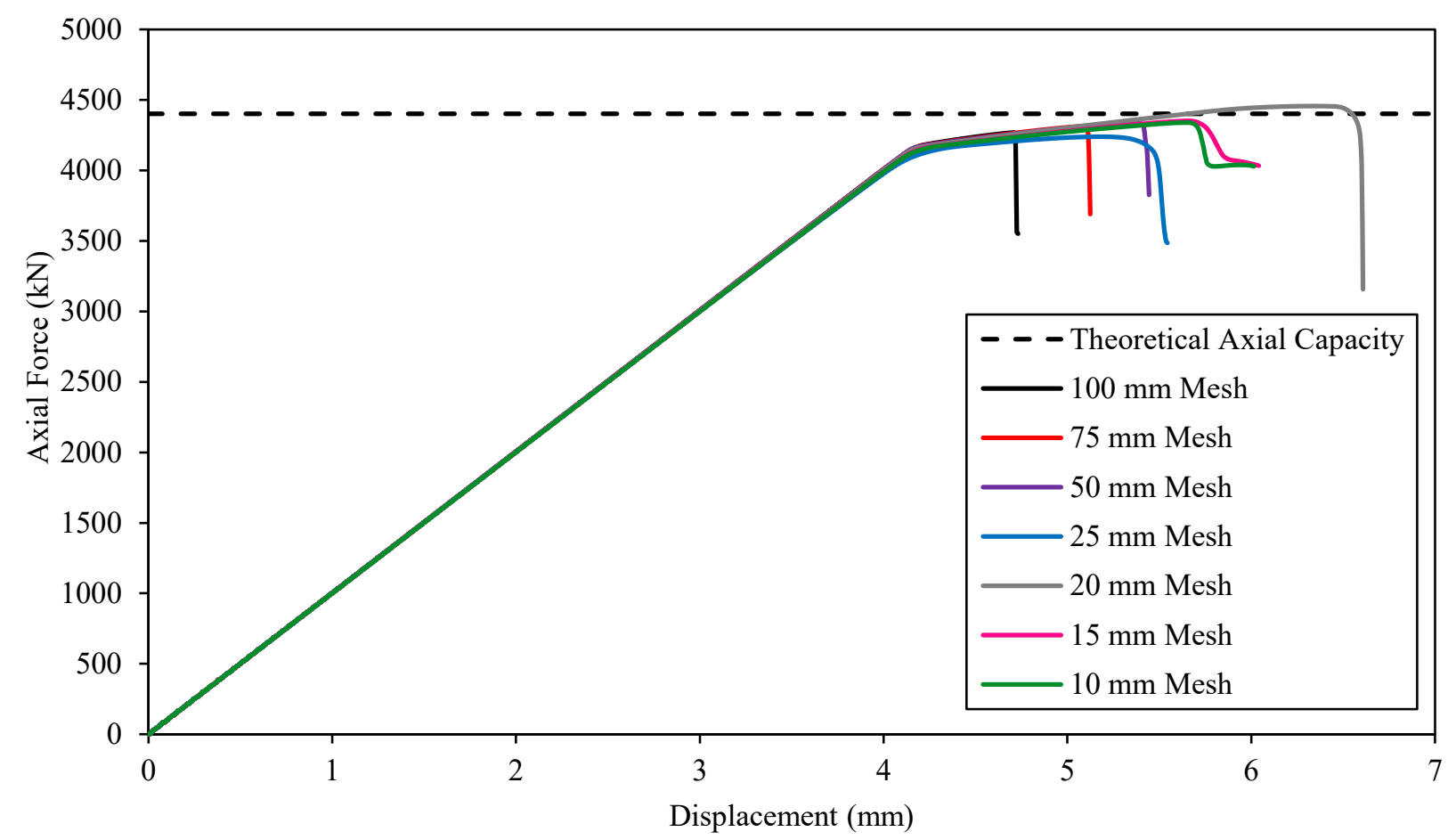

Figure 11: Mesh sensitivity results for axially loaded RC column using CSCM concrete.

Table 2: Analysis times for various mesh sizes of solid concrete elements using CSCM.

\begin{tabular}{c|c|c} 
Mesh Size (mm) & No. of Concrete Elements & Analysis Time (min) \\
\hline 100 & 270 & 2.27 \\
75 & 640 & 2.60 \\
50 & 2160 & 3.07 \\
25 & 17280 & 8.12 \\
20 & 33750 & 16.3 \\
15 & 80000 & 27.5 \\
10 & 270000 & 81.4
\end{tabular}




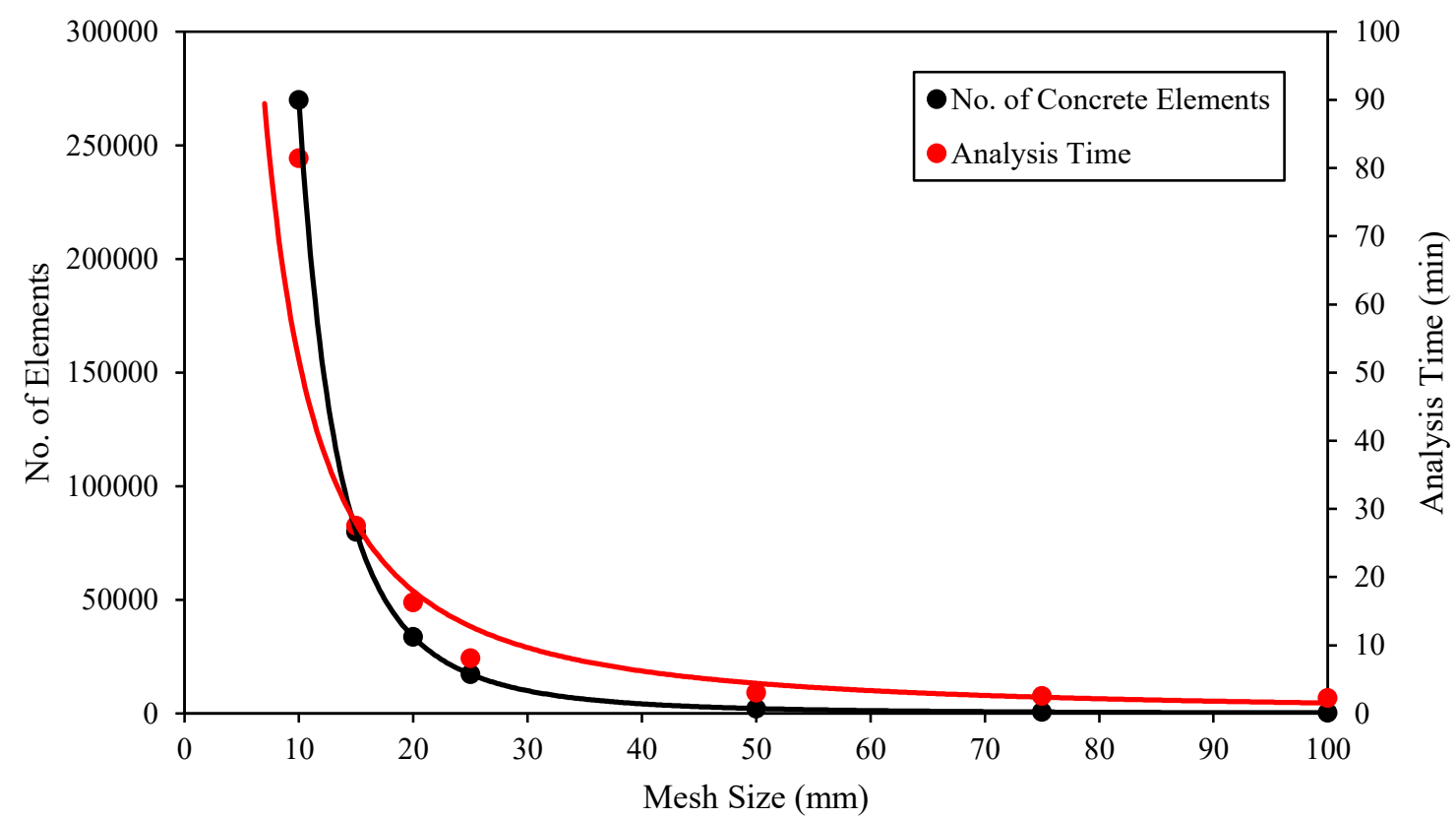

Figure 12: Analysis time and total number of elements for various concrete solid element mesh sizes using CSCM concrete.

\subsubsection{Erosion of Concrete}

Unlike other concrete material models in LS-DYNA, an erosion function is built-in to CSCM through the ERODE parameter. Element erosion in CSCM is defined based on accumulated damage and maximum principal strain in an element. Elements lose all strength and stiffness when the scalar damage parameter, $d$, reaches or exceeds a value of 0.99 . However, elements are deleted from the simulation based on a limiting maximum principal strain value. Figure 13 shows the erosion of a quarter model of an RC slab specimen tested by Wang et al. (2012) which is used for the verification study in this research. The experiment details and corresponding numerical model are discussed in detail in Section 5.2.2. Figure 14 shows the maximum principal strains of four randomly selected elements which were deleted from the analysis through the erosion function. The elements are also highlighted in Figure 13. In this analysis, the ERODE parameter was set to 1.2, which corresponds to a maximum principal strain value of 0.2. As observed in Figure 14, the elements are deleted from the simulation when they accumulate a damage of 0.99 and reach a 
maximum principal strain value of 0.2 , at which point the curve shows a sharp and sudden drop to zero (indicating element deletion).

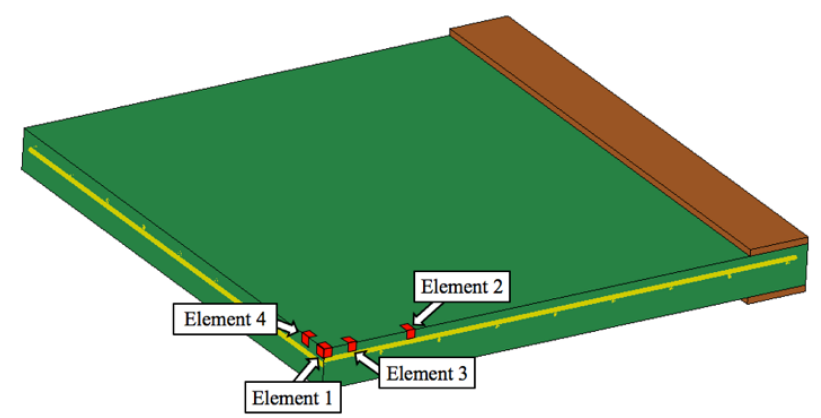

(a)

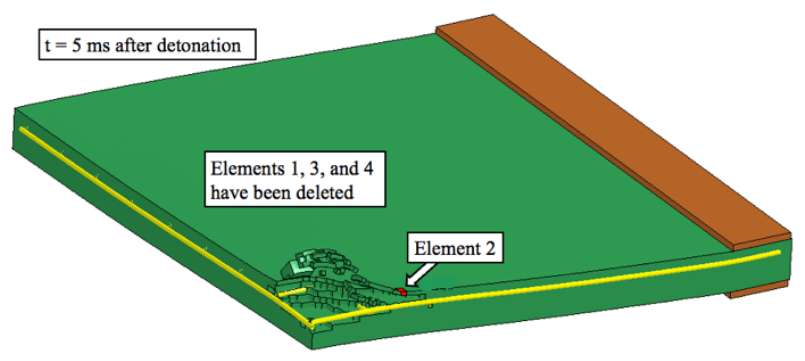

(b)

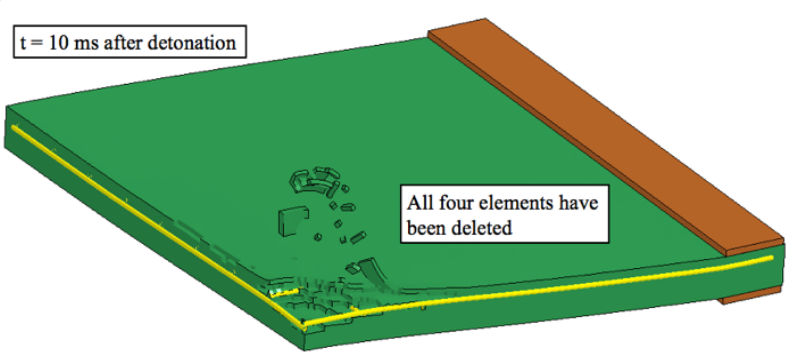

(c)

Figure 13: Quarter model of an RC slab showing erosion of concrete elements (a) prior to blast loading, (b) $5 \mathrm{~ms}$ after detonation, and (c) $10 \mathrm{~ms}$ after detonation.

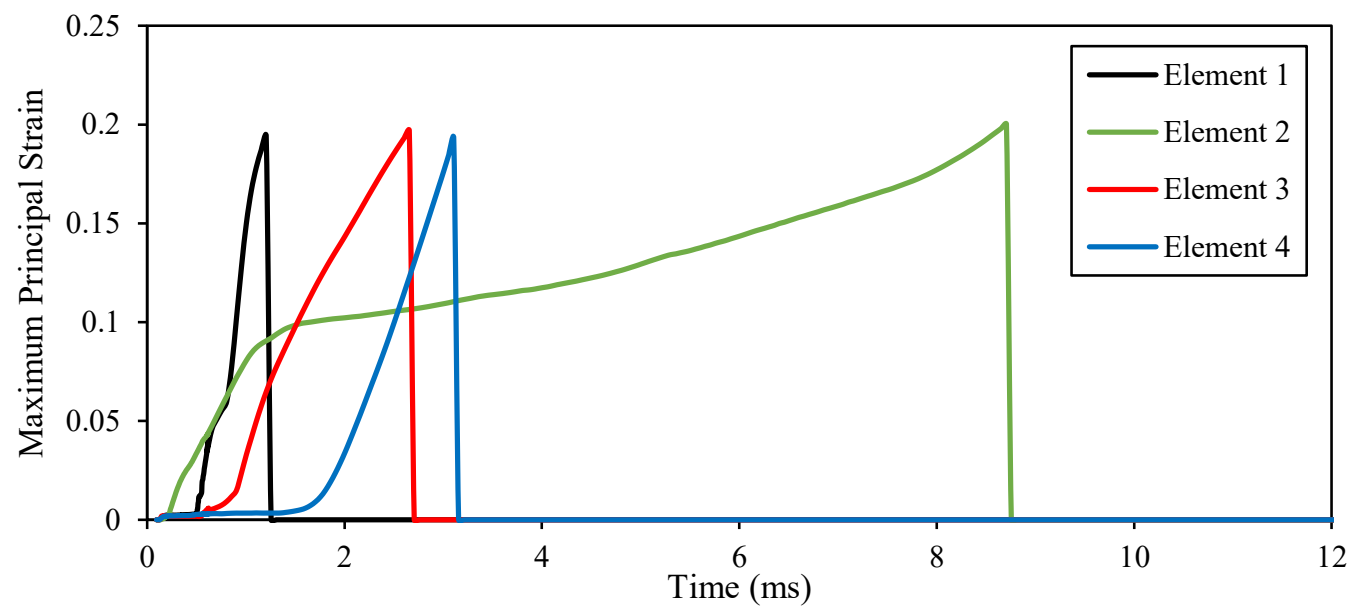

Figure 14: Maximum principal strains for elements deleted by erosion.

Erosion is a numerical modelling technique used to overcome problems associated with excessive mesh distortions. While it may have the appearance of actual material failure or cracks, it does not represent the physical phenomena (Luccioni \& Aráoz, 2011). It is intended to delete elements that have lost all strength and stiffness and prevent computational instability problems. However, often 
in the literature, erosion is calibrated to a specific numerical model to qualitatively resemble damage patterns or spalling. As a result, there is a range of limiting values reported in the literature. For instance, Tang and Hao (2010) used a limiting principal strain value of 0.1. Other erosion criteria are also available, such as shear strain, which was used by Wang et al. (2009), where the limiting value was set to 0.4. Shi et al. (2010) used a combination of shear strain and principal strain criteria, setting the limiting values to 0.9 and 0.15 , respectively.

Selecting an appropriate erosion criterion and limit is critical. Without erosion, severely distorted elements can lead to numerical instabilities and exceedingly small timesteps. Furthermore, these elements which have become severely distorted tend to "lock up", and induce unrealistic distortions in the Lagrangian mesh (Luccioni \& Aráoz, 2011). However, setting the erosion limit too low can lead to excessive deletion of elements and inaccurate results. This can cause loss of internal energy, strength and potentially mass. Therefore, the erosion limit should be selected so that only elements that are severely distorted be deleted from the simulation. As a result, the erosion limit cannot be fixed independently of mesh size (Luccioni \& Aráoz, 2011).

Based on these considerations, as well as the specifics of each model, the ERODE value was set between $1.2-1.4$ for the numerical simulations conducted in this research.

\subsubsection{Reinforcing Steel}

The reinforcing steel was explicitly modelled as $10 \mathrm{~mm}$ beam elements and constrained using the CONSTRAINED_BEAM_IN_SOLID (CBIS) keyword. By constraining the steel beam elements within the concrete, the motion of the two meshes are restrained allowing them to move together (ie. perfect bond assumption), with the concrete serving as the master component. The slave beam elements were coupled to the master concrete elements by constraining the displacement, velocity, and acceleration of the beam nodes. The CONTACT_AUTOMATIC_GENERAL keyword was 
used to define the contact and interaction between longitudinal and transverse reinforcement. This contact type performs checks along the entire length of the beam segment to ensure penetration of one beam element into another does not occur (LSTC, 2017b).

\subsubsection{Material Model}

Steel reinforcing bars were modelled using material type 024 (MAT_PIECEWISE_LINEAR_PLASTICITY). This is an elastoplastic material, which allows for the input of a stress-strain curve as well as strain rate dependency (LSTC, 2017a).

Under high strain rates, steel experiences an increase in both yield strength and ultimate strength. However, as shown in Figure 15, the increase factor for yield strength is far more pronounced than the increase factor for ultimate strength.

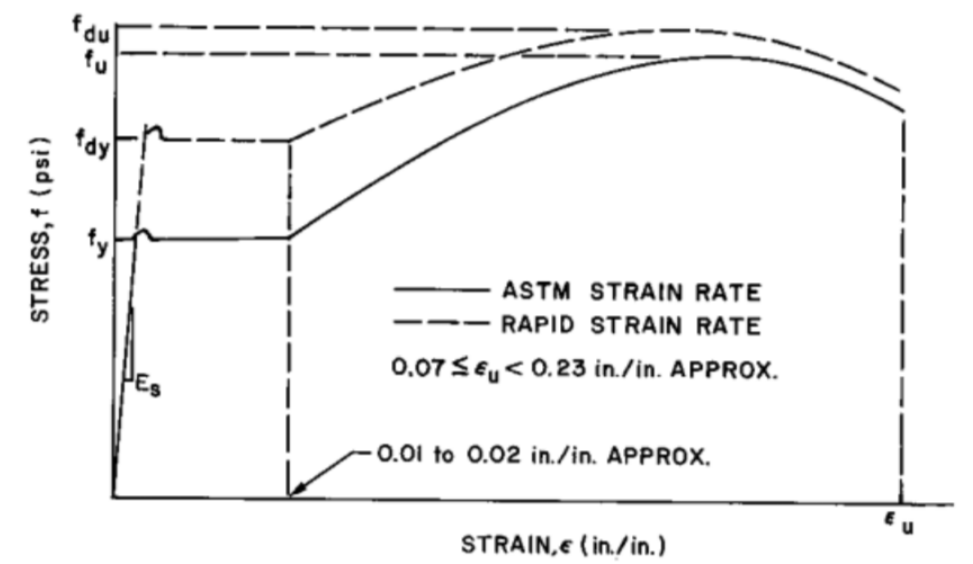

Figure 15: Stress-strain relationship for steel under static and rapid loading (UFC 03-0340-02, 2008).

Rate effects for material type 024 can be user-defined, which allows for the definition of the strain rate effect on the yield stress. In this study, the DIF for the steel reinforcing bars was defined according to Malvar and Crawford (1988) using the following equation:

$$
D I F=\left(\frac{\dot{\varepsilon}}{10^{-4}}\right)^{\alpha}
$$


For the yield stress, $\alpha=\alpha_{f y}$ which is determined as:

$$
\alpha_{f y}=0.074-0.040 \frac{f_{y}}{414}
$$

Equation 3

where $\dot{\varepsilon}$ is the strain rate in $\mathrm{s}^{-1}$ (1/second) and $\mathrm{f}_{\mathrm{y}}$ is the yield strength in MPa.

\subsubsection{Mesh Sensitivity Analysis}

Through a sensitivity analysis, it was found that mesh size of beam elements has a minimal effect on analysis results. The same approach used to evaluate concrete mesh size was used to evaluate steel mesh size of beam elements. The RC column described in Section 4.1.1.2 was again subjected to a displacement-controlled axial load of $0.01 \mathrm{~mm} / \mathrm{ms}$. However, for this set of analyses, the concrete mesh size was kept constant at $15 \mathrm{~mm}$, and the mesh size of the steel beam elements was varied.

The load-displacement results for the RC column using various mesh sizes for steel reinforcement are plotted in Figure 16. As seen in the figure, the mesh size had a minimal effect on the results. The number of steel elements used in the FE model and corresponding analysis times are listed in Table 3 and graphically presented in Figure 17. While the mesh size of the steel beam elements did result in an increase in analysis time, a mesh size of $10 \mathrm{~mm}$ was still used for the steel reinforcing bars in this research. This was done to provide adequate detailed element output data regarding stresses and strains in the steel reinforcement. 


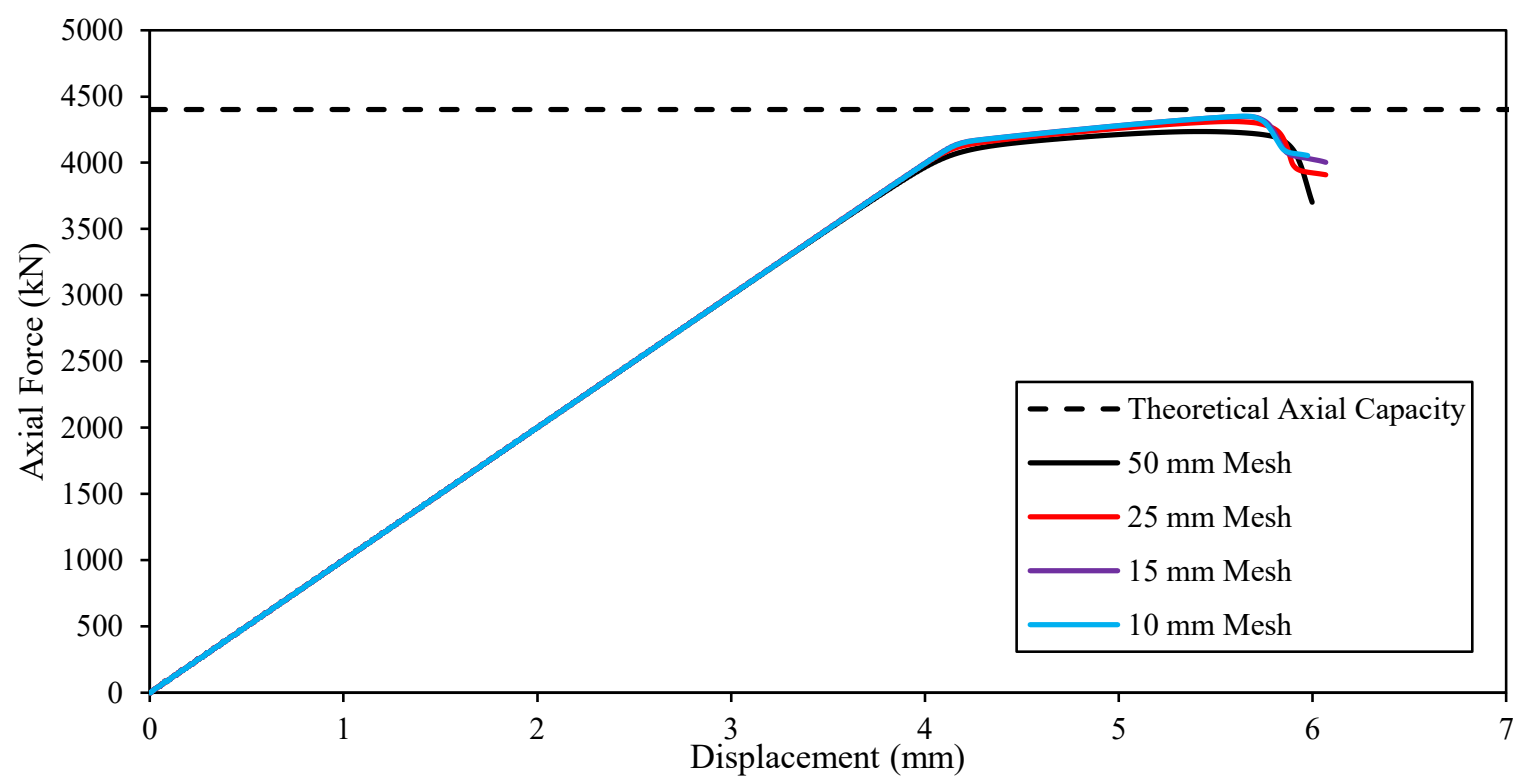

Figure 16: Mesh sensitivity results for beam elements representing steel reinforcing bars using PIECEWISE_LINEAR_PLASTICITY material model.

Table 3: Analysis times for various mesh sizes of beam elements using MAT_PIECEWISE_LINEAR_PLASTICITY.

\begin{tabular}{c|c|c} 
Mesh Size (mm) & No. of Steel Elements & Analysis Time (min) \\
\hline 50 & 612 & 9.18 \\
25 & 1192 & 9.48 \\
15 & 2052 & 11.6 \\
10 & 3008 & 27.5
\end{tabular}

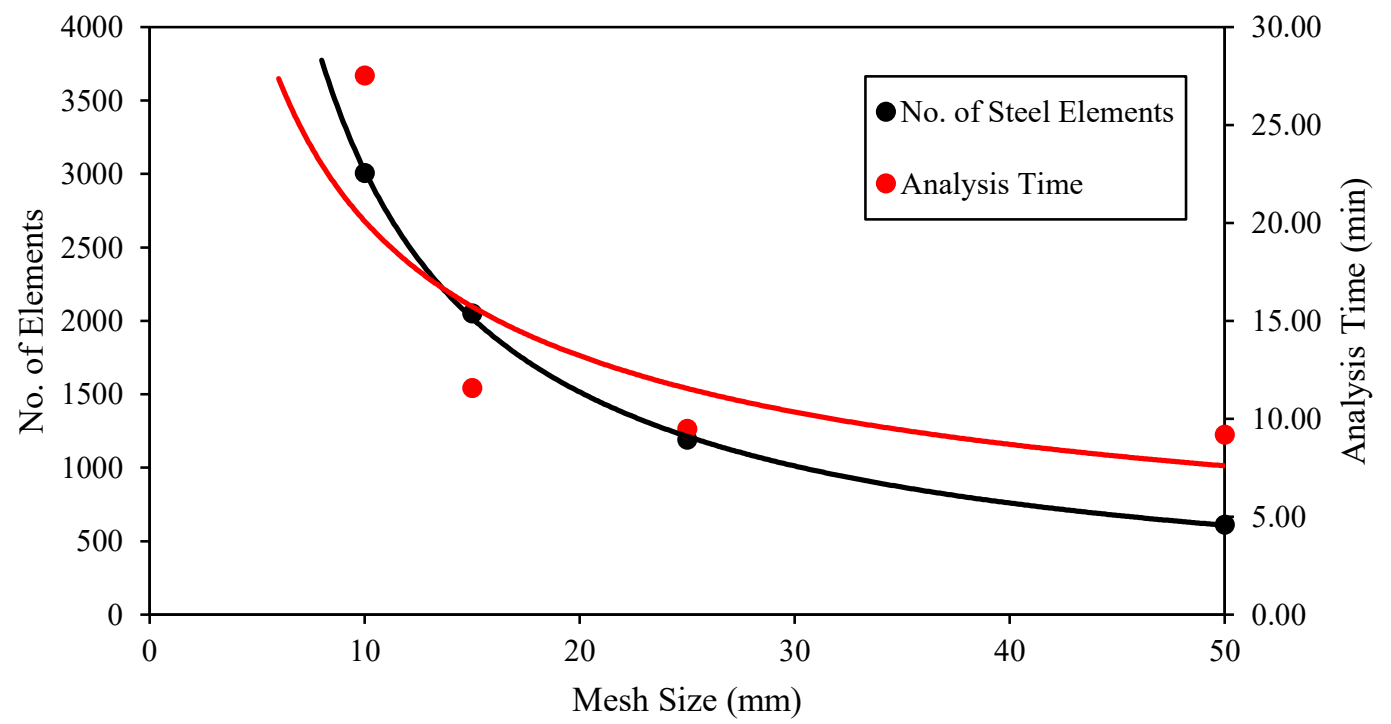

Figure 17: Analysis times and total number of elements for various beam element mesh sizes using MAT_PIECEWISE_LINEAR_PLASTICITY. 


\subsubsection{Support Conditions}

End support conditions can either be modelled using nodal constraints directly applied to the structural member or the physical supports may be modelled. When validating the FE model against experiments, modelling the support system provided more accurate results. Because achieving fully fixed supports in field experiments can be difficult, the degree of fixity is often unknown and typically is somewhat less than fully fixed condition. The use of nodal constraints in the FE model tended to induce too much constraint and underestimate displacements, while modelling the support structure with proper contact modelling provided more realistic boundary conditions leading to better estimation of structural response.

In order to model the contact and interaction between the steel support plates and concrete specimen, the keyword CONTACT_AUTOMATIC_SURFACE_TO_SURFACE was used. Depicted in Figure 18 below, this contact type transmits compressive and tangential loads (sliding) between segments and checks for penetrations of elements between master and slave components (LSTC, 2017b). This method was used for both simply supported and fixed end conditions. Figure 18 shows a simply supported end condition, where the slab is allowed to freely move and interact with the steel plates as it responds to the blast load. For a fully fixed end condition, an additional steel plate was modelled above the specimen to prevent translation and rotation. 


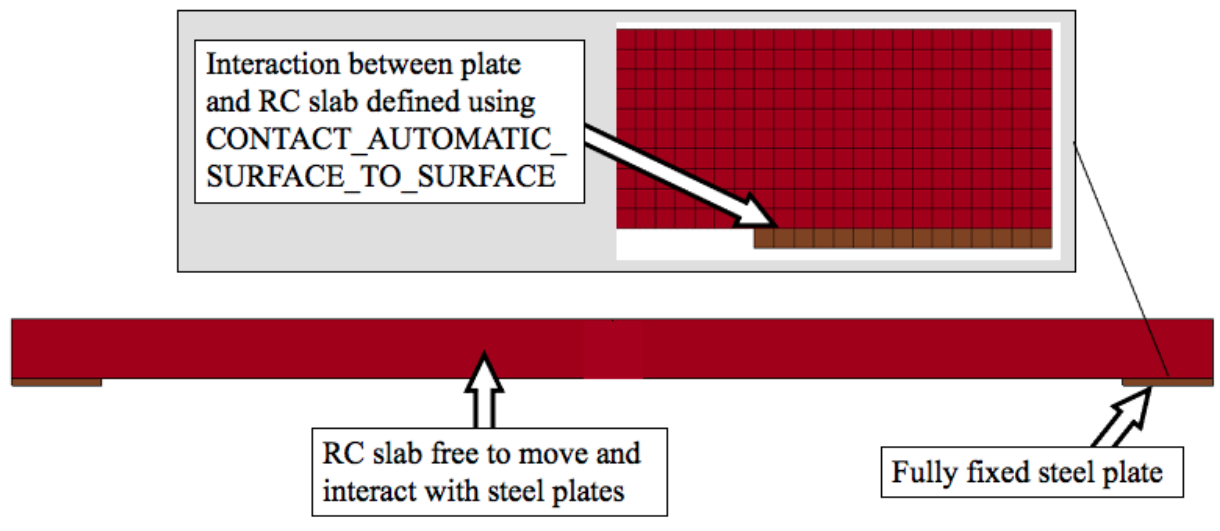

(a)

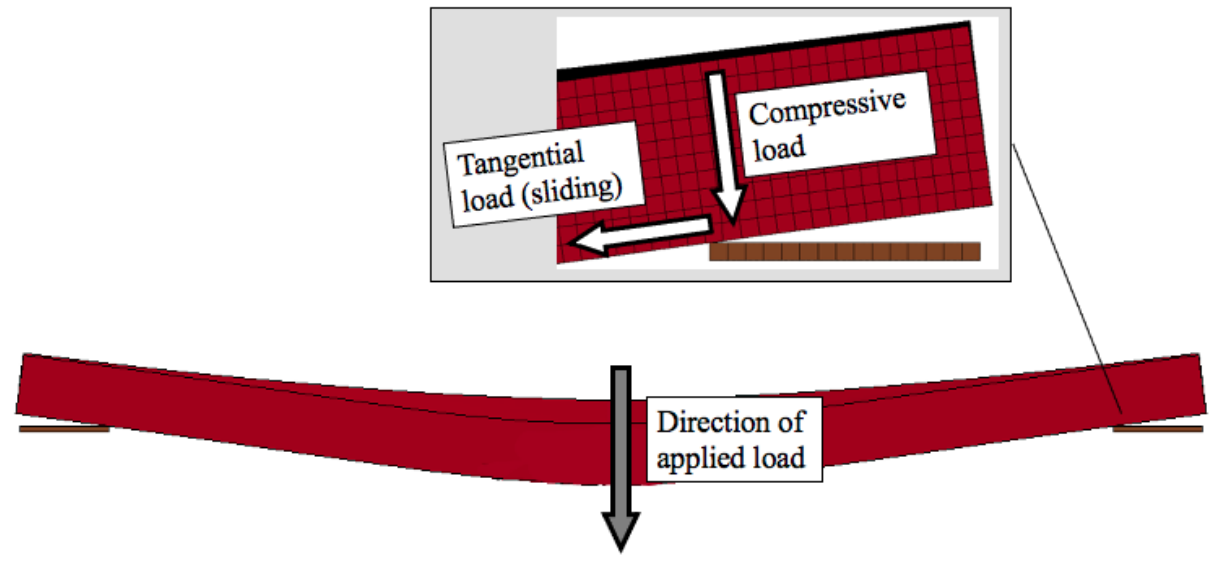

(b)

Figure 18: Interaction between two Lagrangian entities (steel supports and an RC slab) using CONTACT_AUTOMATIC_SURFACE_TO_SURFACE (a) prior to loading application and (b) after loading application.

\subsubsection{Axial Loading}

Axial loading was applied and distributed to the nodes on the top surface of the column using the LOAD_SEGMENT_SET keyword, as shown in Figure 19. It was applied prior to the application of the blast load as a constant load. Additionally, residual axial capacity was measured by increasing the axial loading until complete failure of the blast-damaged columns. 


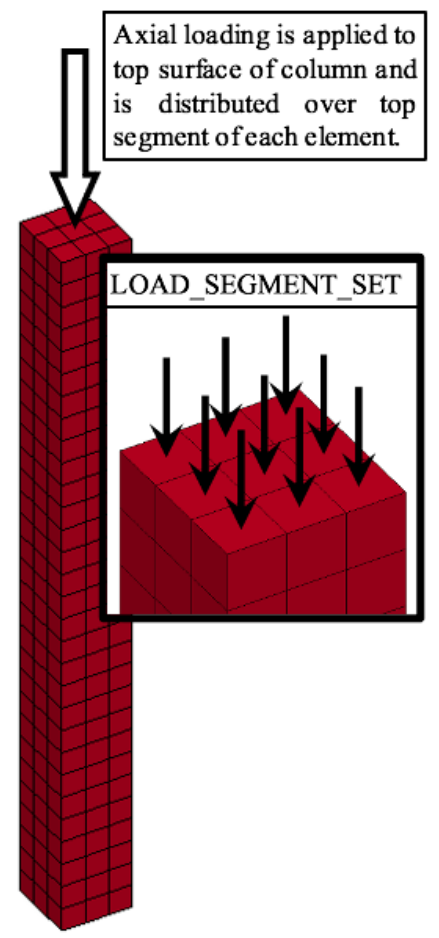

Figure 19: Application of axial loading to RC columns.

\subsubsection{Damping}

In blast analysis, damping tends to have very little effect on the peak response of the structure. Due to the very short duration of loading, the maximum response typically occurs within the first cycle of the structure's response, before the damping forces can absorb much energy from the structure. Furthermore, the energy that is dissipated through plastic deformation is significantly greater than that dissipated by structural damping (Figuli et al., 2008; U.S. Army Corps of Engineers, 2008). In design, damping is often neglected, as it simplifies calculations and serves as a more conservative approach, but the inclusion of damping can provide more accurate results (Carlsson \& Kristensson, 2012). PEC and BakerRisk (2008) suggest that a small damping ratio of about $1 \%$, should be used in blast analysis. Therefore, the damping ratio in this research was set to $1 \%$ using the DAMPING_GLOBAL keyword in LS-DYNA. 


\subsection{Air and Explosive Modelling}

\subsubsection{General}

An Arbitrary Lagrangian-Eulerian (ALE) formulation is used to provide a stable and accurate coupling algorithm to solve the fluid-structure interaction problem. By adopting this modelling technique, both the blast wave propagation and its interaction with the structure are modelled. In a typical Lagrangian formulation, the mesh moves with the material. While it is commonly used to solve problems in solid mechanics, large mesh distortions can result in numerical instability. In a Eulerian formulation, the mesh is fixed in space. Used often in fluid mechanics, it introduces an additional complexity of having to define convective terms for material to move through the mesh (Souli \& Benson, 2010). Neither formulation is appropriate to be used for the entire domain of a fluid-structure interaction problem.

Multi-material ALE (MM-ALE) allows for several fluids to interact with one another and the Lagrangian structure. The MM-ALE formulation allows the mesh containing different fluids to move in an arbitrary manner. It conforms to the Lagrangian mesh along the boundary of the structure while remaining fixed elsewhere. The MM-ALE formulation is employed in this research study.

\subsubsection{Modelling of Air}

\subsubsection{Material Model and EOS}

Material type 009 (MAT_NULL) was used to model the air. This model requires the mass density of the material and is intended to be used for materials that behave in a fluid-like manner. It also allows for the consideration of equations of state (EOS) (LSTC, 2017b). 
In order to define the fluid flow characteristics of the air, a simplified linear polynomial equation of state (EOS_LINEAR_POLYNOMIAL) was used. The pressure, $P$, is defined by the following equation:

$$
P=C_{0}+C_{1} \mu+C_{2} \mu^{2}+C_{3} \mu^{3}+\left(C_{4}+C_{5} \mu+C_{6} \mu^{2}\right) E \quad \text { Equation } 4
$$

where $C_{n}$ are material parameters, $\mu=\rho / \rho_{0}-1$, with $\rho / \rho_{0}$ being the ratio of current density to initial density, and $E$ is the energy per initial unit volume.

The air is assumed to be an ideal gas, and the gamma law EOS is used, via the following:

$$
\begin{aligned}
C_{0}=C_{1} & =C_{2}=C_{3}=C_{6}=0 & & \text { Equation } 5 \\
C_{4} & =C_{5}=\gamma-1 & & \text { Equation 6 }
\end{aligned}
$$

where $\gamma$ is the ratio of specific heats and can be determined from the following equation:

$$
\gamma=\frac{C_{p}}{C_{v}}=\frac{1.005 \mathrm{~kJ} / \mathrm{kg} \cdot \mathrm{K}}{0.718 \mathrm{~kJ} / \mathrm{kg} \cdot \mathrm{K}}=1.400
$$

Equation 7

where $C_{p}$ is the specific heat at constant pressure and $C_{v}$ is the specific heat at constant volume (given a temperature of $300 \mathrm{~K}$ for air).

The EOS parameters for the air can be found in Table 4, where $\rho$ is the mass density, $C_{n}$ represents the polynomial equation coefficients, $E_{0}$ is the initial internal energy, and $V_{0}$ is the initial relative volume.

Table 4: Material and EOS parameters for air.

\begin{tabular}{c|c|c|c|c|c|c|c|c|c}
$\boldsymbol{\rho}\left(\mathbf{k g} / \mathbf{m m}^{\mathbf{3}}\right)$ & $\mathbf{C}_{\mathbf{0}}$ & $\mathbf{C}_{\mathbf{1}}$ & $\mathbf{C}_{\mathbf{2}}$ & $\mathbf{C}_{\mathbf{3}}$ & $\mathbf{C}_{\mathbf{4}}$ & $\mathbf{C}_{\mathbf{5}}$ & $\mathbf{C}_{\mathbf{6}}$ & $\mathbf{E}_{\mathbf{0}}(\mathbf{G P a})$ & $\mathbf{V}_{\mathbf{0}}$ \\
\hline $1.225 \times 10^{-9}$ & 0 & 0 & 0 & 0 & 0.4 & 0.4 & 0 & $2.5 \times 10^{-4}$ & 1
\end{tabular}

\subsubsection{Modelling of Explosive}

\subsubsection{Material Model and EOS}

Material type 008 (MAT_HIGH_EXPLOSIVE_BURN) was used to model the explosive. It takes into account the density, $\rho$, detonation velocity, $D$, and the Chapman-Jouguet pressure, $P_{C J}$. This 
material model is intended to model the detonation of a high explosive in conjunction with an equation of state (EOS).

The time and location of detonation of the explosive were governed by the keyword INITIAL_DETONATION, while the size and shape of the explosive were defined using the INITIAL_VOLUME_FRACTION_GEOMETRY keyword. This command allows for modelling of various explosive charge shapes, which include spherical and cylindrical charges. This is particularly important as the explosive shape can affect pressure distributions in the near-field (Hao et al., 2016).

The Jones-Wilkins-Lee (JWL) EOS was used to describe the flow of the detonation products of the high explosive. It defines pressure, $p$, as:

$$
p=A\left(1-\frac{\omega}{R_{1} V}\right) e^{-R_{1} V}+B\left(1-\frac{\omega}{R_{2} V}\right) e^{-R_{2} V}+\frac{\omega E}{V} \quad \text { Equation } 8
$$

where, $V$ is the volume of explosive material relative to the container size, and $A, B, R_{1}, R_{2}$, and $\omega$, are constants which depend on the type of explosive material.

The material model and EOS parameters for TNT, ammonium nitrate/fuel oil (ANFO), and plastic explosive (PE4) are given in Table 5 and Table 6.

\begin{tabular}{c|c|c|c}
\multicolumn{2}{|c|}{ Table 5: Material parameters for explosives. } & \\
Explosive & $\boldsymbol{\rho}\left(\mathbf{k g} / \mathbf{m m}^{\mathbf{3}}\right)$ & $\mathbf{D}(\mathbf{m m} / \mathbf{m s})$ & PCJ $_{\mathbf{G P a})}$ \\
\hline TNT & $1.630 \times 10^{-6}$ & 6930 & 21 \\
PE4 & $1.601 \times 10^{-6}$ & 8193 & 28 \\
ANFO & $6.500 \times 10^{-7}$ & 2200 & 3
\end{tabular}

Table 6: EOS parameters for explosives.

\begin{tabular}{c|c|c|c|c|c|c|c} 
Explosive & $\mathbf{A}(\mathbf{G P a})$ & $\mathbf{B}(\mathbf{G P a})$ & $\mathbf{R}_{\mathbf{1}}$ & $\mathbf{R}_{\mathbf{2}}$ & $\boldsymbol{\omega}$ & $\mathbf{E}_{\mathbf{0}}(\mathbf{G P a})$ & $\mathbf{V}_{\mathbf{0}}$ \\
\hline $\mathrm{TNT}$ & 373.8 & 3.747 & 4.15 & 0.90 & 0.35 & 7.00 & 1 \\
PE4 & 609.8 & 12.95 & 4.50 & 1.40 & 0.25 & 9.00 & 1 \\
ANFO & 491.0 & 0.890 & 3.90 & 1.18 & 0.33 & 2.48 & 1
\end{tabular}




\subsubsection{Mapping Technique}

A mapping technique was used to increase the efficiency of the modelling and reduce the computational cost. Typically, this mapping technique was completed in a $2 \mathrm{D}$ to a 3D environment. The initial detonation process of the charge and blast wave propagation was modelled in a 2D environment with only the MM-ALE domain. This simulation was terminated prior to the blast wave impingement on the structure, and a mapping file was created, which included nodal and element data from the last cycle of the analysis. This data was then mapped onto a coarser 3D MM-ALE mesh along with the Lagrangian domains. The analysis was allowed to continue until the complete interaction of the blast wave and the structure. Once the blast wave had passed the structure entirely and the coupling forces reduced to a negligible value, the analysis was terminated once more. The ALE elements and related commands were removed from the model, and a full-deck restart was executed using the STRESS_INITIALIZATION command (LSTC, 2017b). The structure continued its response in the free-vibration stage until it came to rest.

When the charge mass was cylindrical in shape, a 3D-to-3D mapping process was used. This was done to simplify the modelling of the cylinder. Rather than model the charge as a separate part in $2 \mathrm{D}$ and then mesh the interface between the air and the charge, the cylinder was modelled in 3D using the default conical container geometry which requires the definition of two end points and two corresponding radii. In a similar manner, the explosive was detonated in a $3 \mathrm{D}$ model containing only the air and explosive parts in the MM-ALE domain. Prior to impingement on the structure the data was mapped into a second 3D model with a coarser air mesh that contained the structure. 
The mapping technique significantly reduces the computational cost and analysis time. It ensures smaller file sizes without compromising the integrity of the results. Analysis run times are significantly shorter and size of data storage is reduced.

\subsubsection{Domain Modelling}

For spherical charge mass shapes, the air domain was modelled in 2D as a quarter-symmetric circular shell with a maximum air mesh size of $2 \mathrm{~mm}$. This ensures that the initial detonation process and the initial energy is accurately determined (Lapoujade et al., 2010).

To model the MM-ALE element formulation in 2D, axisymmetric quadrilateral elements were used with boundary conditions prescribed along the radial edges of the quarter circle to ensure displacement compatibility requirements due to symmetry in the appropriate directions were met. The 2D elements lie in the $x-y$ plane and have two degrees of freedom per node (displacement in the $\mathrm{x}$ and $\mathrm{y}$ directions). Symmetry is frequently used in the presented research in order to reduce the number of elements and improve computational performance. Figure 20 presents the $2 \mathrm{D} \mathrm{FE}$ model as well as the contours of the blast pressure at a time 0.09 milliseconds after detonation of $1 \mathrm{~kg}$ spherical TNT.

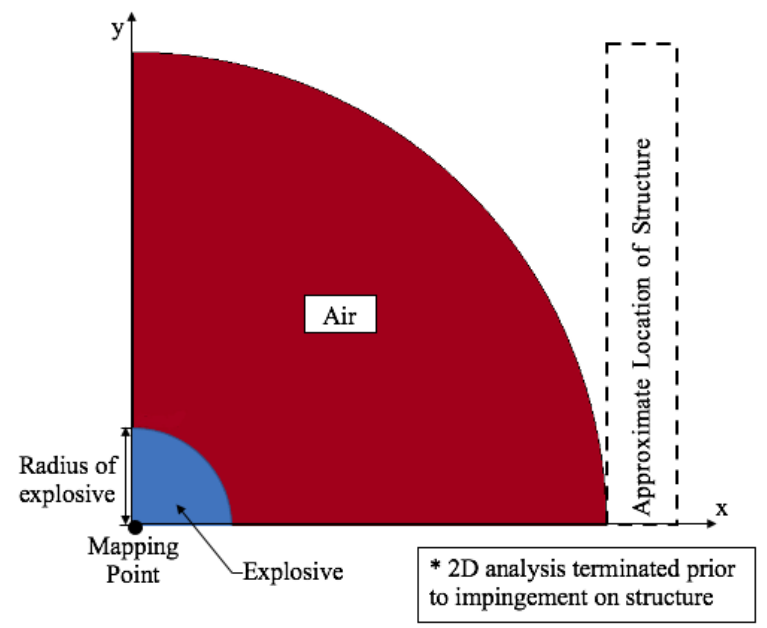

(a)

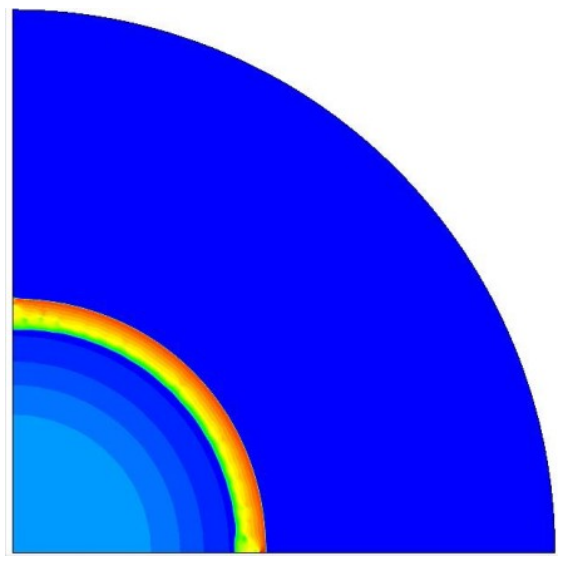

Pressure (GPa)

7.171e-03

$6.464 \mathrm{e}-03$

$5.757 \mathrm{e}-03$

$5.049 \mathrm{e}-03$

4.342e-03

$3.635 \mathrm{e}-03$

$2.928 \mathrm{e}-03$

$2.220 \mathrm{e}-03$

$1.513 \mathrm{e}-03$

$8.060 \mathrm{e}-04$

9.881e-05

(b)

Figure 20: (a) Air and explosive in the 2D model (b) Blast pressure propagation in 2D. 
Figure 21 and Figure 22 present the results of a mesh sensitivity analysis of the detonation process for $1 \mathrm{~kg}$ of TNT modelled in the 2D environment. The pressures were collected $300 \mathrm{~mm}$ from the point of detonation resulting in a scaled distance of $z=0.3 \mathrm{~m} / \mathrm{kg}^{1 / 3}$. Based on the analysis results, both the pressure and impulse values converge at a mesh size of $2 \mathrm{~mm}$. Further decreasing the mesh size has a negligible effect on the pressure and impulse generated, but significantly increases both the number of elements and the analysis time, as shown in Table 7 and Figure 23. Based on these results, a minimum mesh size of $2 \mathrm{~mm}$ was used to model the detonation of the explosives.

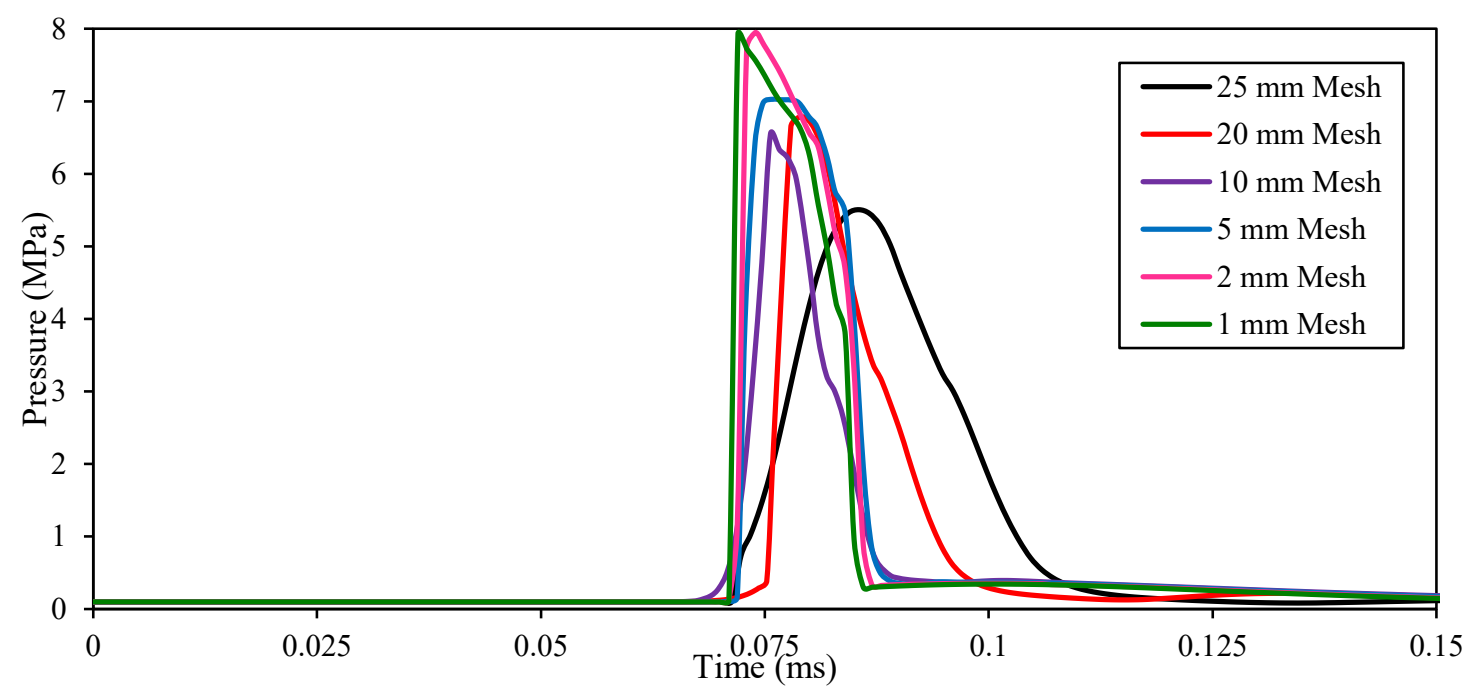

Figure 21: Mesh sensitivity analysis of overpressures generated during the detonation process.

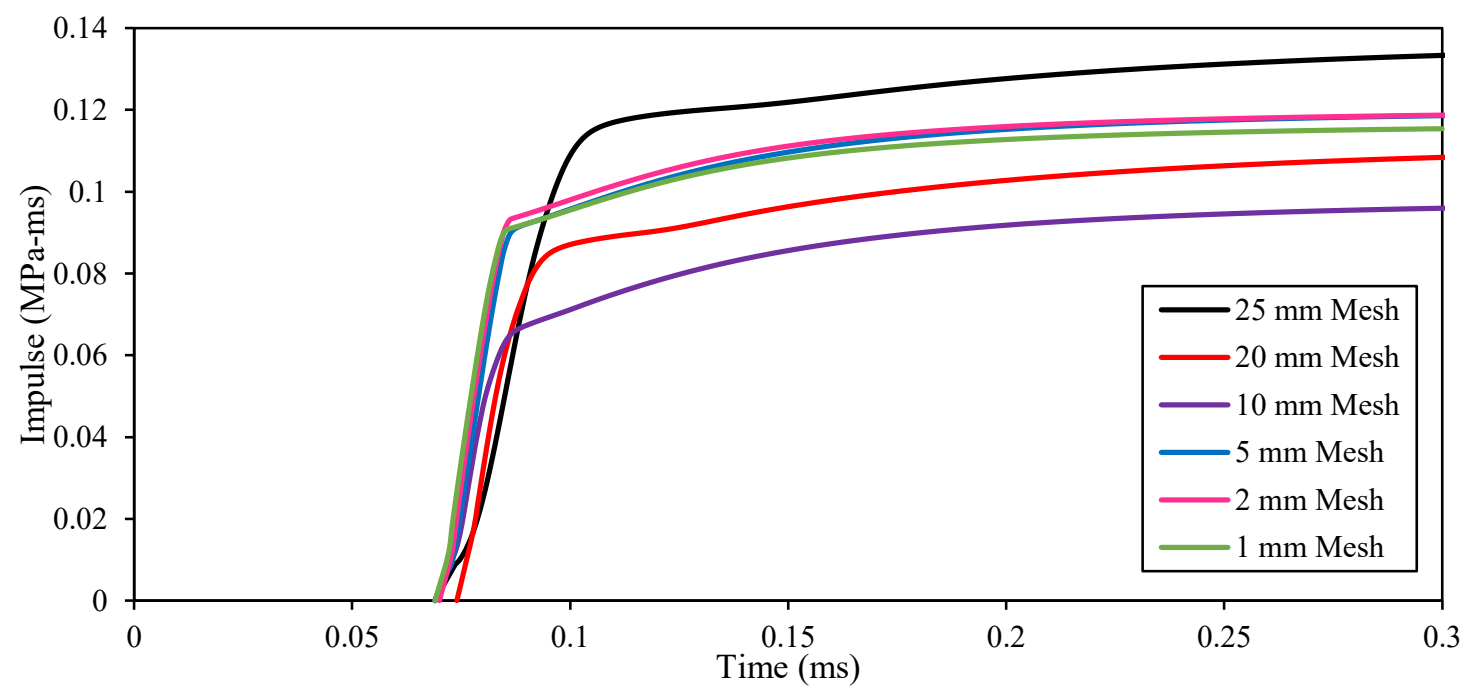

Figure 22: Mesh sensitivity analysis of the impulse generated during the detonation process. 
Table 7: Analysis times and total number of elements for various 2D air mesh sizes.

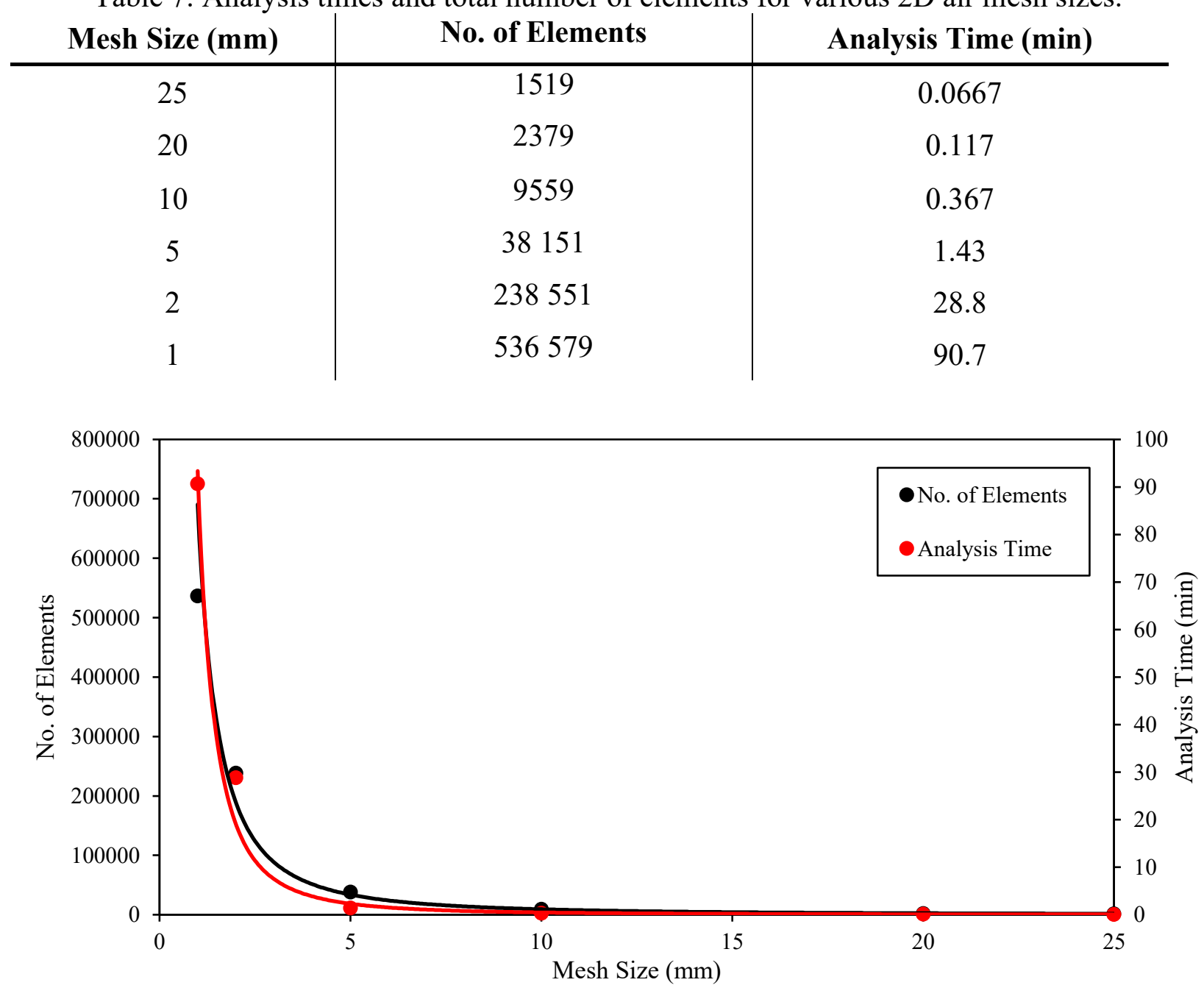

Figure 23: Analysis time and total number of elements for various 2D air mesh sizes.

For detonation of cylindrical charge mass shapes, the air domain was modelled in 3D as a box using eight-node hexahedron solid elements. A minimum mesh size of $2 \mathrm{~mm}$ was used for this research. Figure 24 presents an example of the 3D FE model of the air and explosive as well as a fringe plot of the blast pressure at a time 0.093 milliseconds after detonation of $0.75 \mathrm{~kg}$ cylindrical TNT. 


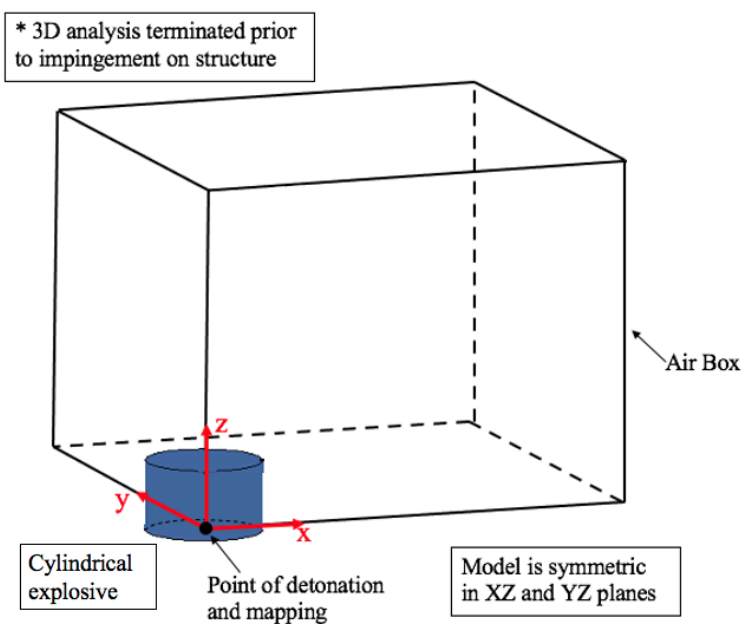

(a)

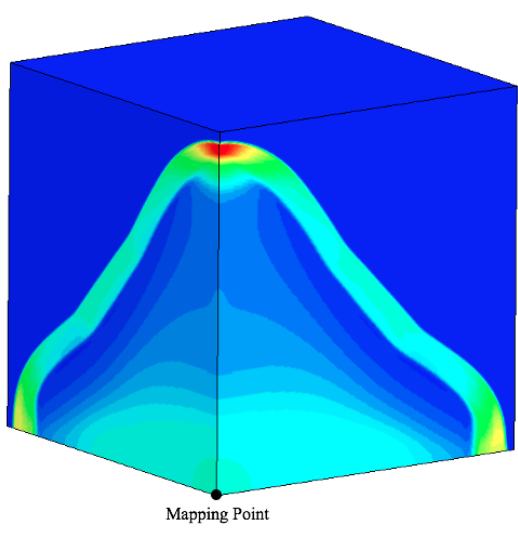

Pressure (GPa)

$1.487 \mathrm{e}-02$

$1.339 \mathrm{e}-02$

$1.191 \mathrm{e}-02$

$1.044 \mathrm{e}-02$

$8.960 \mathrm{e}-03$

7.483e- 03

$6.005 \mathrm{e}-03$

$4.528 \mathrm{e}-03$

$3.051 \mathrm{e}-03$

$1.573 \mathrm{e}-03$

$9.618 \mathrm{e}-05$

(b)

Figure 24: (a) 3D model of air and explosive (b) Blast pressure propagation in 3D.

After explosive detonation and propagation just prior to structure impingement, the data was then mapped into a second 3D model. This model simulated the interaction of the blast wave with the structure, and the air domain was modelled using eight-node hexahedron solid elements $20 \mathrm{~mm}$ in size. A sensitivity analysis to determine this mesh size was conducted during the validation stage and is presented in Section 5.2.1.3.

The size of the air box in 3D is an important consideration as it is necessary to make the air box large enough to prevent the blast wave from reflecting off the boundaries and re-interacting with itself or the structure. The size of the air box is dependent on the specific problem characteristics and therefore is variable with no explicit standard regarding its size. In FE modelling, nonreflecting (flow-out) boundaries can typically be used to prevent this issue. However, these are not compatible with the MM-ALE formulation and incorrect usage of these boundaries can result in significant energy losses. 


\subsubsection{Fluid-Structure Interaction (FSI)}

Fluid-structure interaction (FSI) of the air and explosive with the RC structure was defined using the CONSTRAINED_LAGRANGE_IN_SOLID (CLIS) keyword. This keyword provides a coupling mechanism between the MM-ALE fluids (the air and the explosive) and the Lagrangian entities (the structure). It is a complex keycard that is sensitive to a number of parameters and can significantly affect the results.

The MM-ALE fluids were was defined as the master, while the Lagrangian structure was defined as the slave. One of the sensitive parameters of the CLIS keyword is NQUAD which specifies the number of coupling points that are distributed over each coupled segment. The selection of an appropriate NQUAD value was important and was determined based on relative resolutions of ALE and Lagrangian meshes as well as model calibration in regard to material leakage and model instability. Too few coupling points would result in leakage of ALE material into the Lagrangian elements, while too many would increase computation time and result in numerical instability (Day, 2009).

The method of coupling used for the CLIS keyword also affect the analysis results. The coupling type was selected as penalty coupling with permission of erosion in Lagrangian entities (this being the RC structure). The direction of the coupling was set to occur in the compression direction only. This was selected as it tends to be more numerically stable and robust (LSTC, 2017b). Furthermore, strong leakage controls were included to minimize the degree of leakage of ALE materials into the Lagrangian elements.

Figure 25 shows the coupling of ALE material to a steel plate. In Figure 25a, the explosive material is passing through the plate. This behaviour is referred to as leakage and can result in inaccuracies. 
Through proper definition of the CLIS keyword and the inclusion of leakage controls, the leakage can be minimized or eliminated, as observed in Figure 25b.

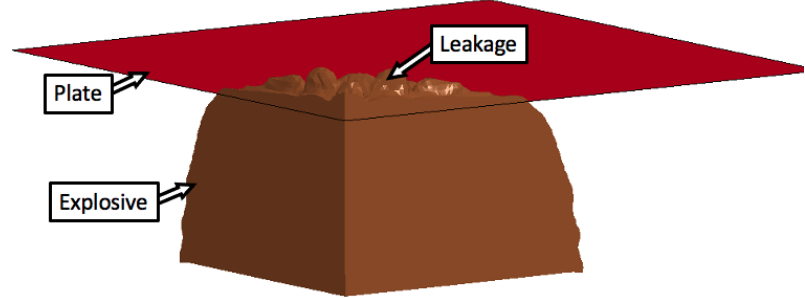

(a)

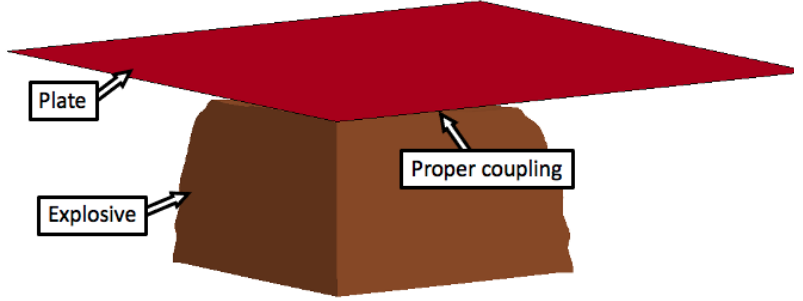

(b)

Figure 25: Coupling of ALE material to a Lagrangian plate showing (a) poor coupling with leakage and (b) proper coupling and no leakage. 


\section{Model Validation}

The numerical model was verified in two main phases against experimental data reported from near-field tests. Experimental work in blast is uniquely challenging due to high experimentation costs and unpredictable conditions that can be difficult to control. Equipment can be easily damaged or thrown out of place during field tests. As a result, the number of reliable experimental studies on blast that are available in open literature are limited. Furthermore, because of the inherent uncertainties in blast experiments, a larger margin of error is deemed acceptable when validating FE models in blast. To compensate for these uncertainties, the FE model used in this research was verified against a number of independent experiments to ensure its ability to predict blast loading and structural response in the near-field.

For the first phase of the validation, the FE model was validated against two separate field experiments to confirm its ability to predict pressure and impulse at scaled distances below 0.4 $\mathrm{m} / \mathrm{kg}^{1 / 3}$. For the second phase of the validation, the ability of the model to predict structural response under near-field blast loading was validated against three separate experiments. As reliable testing data on RC columns under near-field blast loading is limited, the response of the structure was validated using RC slabs and an RC beam, for which the experimental data was readily available in open literature. Specifically, there is no experimental data regarding RC columns subjected to blast loads with a scaled distance less than $0.4 \mathrm{~m} / \mathrm{kg}^{1 / 3}$ that also include axial loading. This absence of experimental data further supports the need for numerical investigations in this area of research. However, the validation using an RC beam will provide verification of the model's ability to simulate global behaviour in a column without axial loading. Column behaviour with axial loading under near-field blast loads is investigated numerically in the parametric study. 
Under near-field blast loading, local damage is perhaps the most important consideration in evaluating the performance of a structure. Local damage primarily depends on material properties and cross-section characteristics (reinforcement details, dimensions, etc.) rather than the type of structural member (Li \& Hao, 2014). As a result, validating the FE model against RC slab and beam specimens will provide a good measure of the ability of the model to capture local damage in RC columns for which reliable test data is not available.

\subsection{Validation of Blast Wave Propagation Modelling in Near-Field}

\subsubsection{Validation against Rigby et al. (2014) Tests}

\subsubsection{Experiment Details}

Rigby et al. (2014) presented a set of experimental results that measured overpressures in the nearfield. Figure 26 shows the test setup used in the study. The experiment was conducted using an array of Hopkinson pressure bars. The bars were fitted through holes of a $100 \mathrm{~mm}$ thick steel target plate which was fully fixed from translation and rotation. Five bars were arranged such that one was located at the centre of the plate (Bar 5 in Figure 26b) and four bars were located $100 \mathrm{~mm}$ radial distance from the centre (Bars 1-4 in Figure 26b). The central bar collected normally reflected pressures, while the four radial bars collected obliquely reflected pressures.

A total of six tests were conducted, each using a $100 \mathrm{~g}$ spherical PE4 charge, which was detonated at a distance of $75 \mathrm{~mm}$ from the centre of the charge to the bottom of the plate. The scaled distance

of the central bar was $0.15 \mathrm{~m} / \mathrm{kg}^{1 / 3}$. The scaled distance of each radial bar was $0.25 \mathrm{~m} / \mathrm{kg}^{1 / 3}$ with an angle of incidence, $\alpha$, of $53^{\circ}$. The angle of incidence is defined as the angle measured between the outward normal of the reflecting surface and a radial line extending from the charge to the point of incidence. An angle of incidence of $0^{\circ}$ results in a normally reflected blast wave (as 
recorded by Bar 5) while an angle of incidence greater than $0^{\circ}$ but less than $90^{\circ}$ results in an obliquely reflected blast wave (as recorded by Bars 1-4).

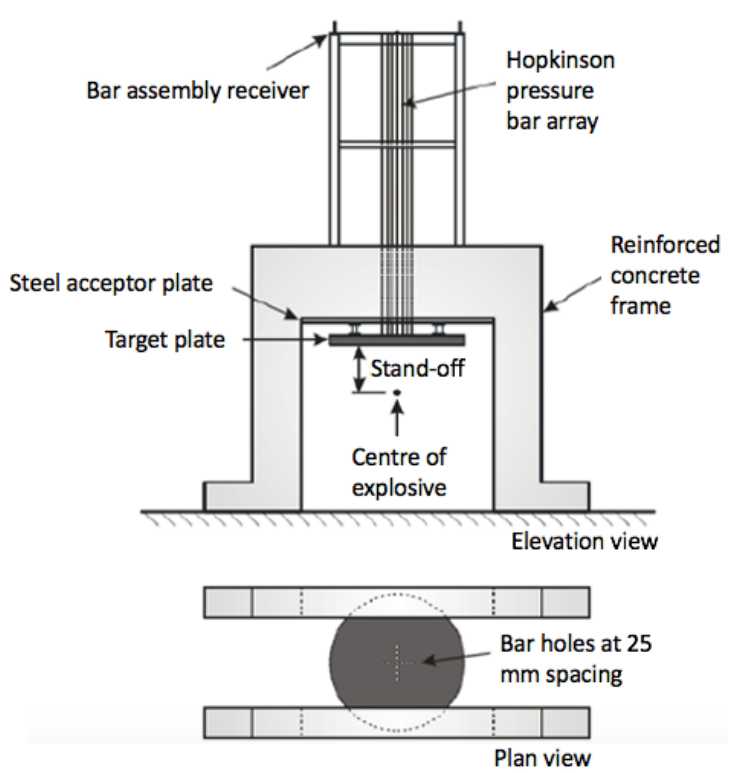

(a)

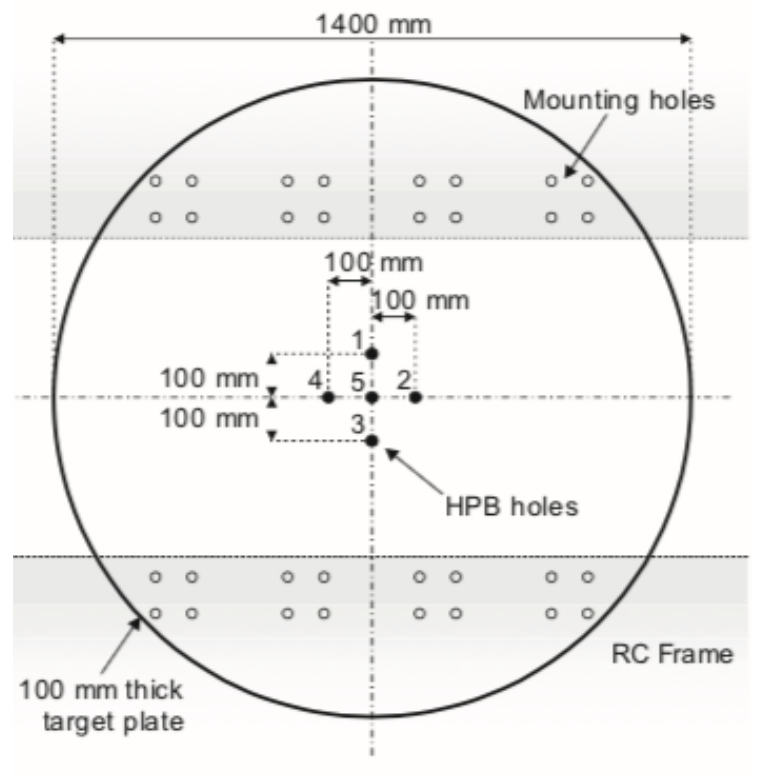

(b)

Figure 26: Test arrangement used to collect overpressure-time histories. (a) Schematic showing overall test setup and (b) Hopkinson bar configuration (Rigby et al., 2015).

\subsubsection{FE Model}

In order to replicate the experiment, a quarter-symmetric model was created in LS-DYNA using the $2 \mathrm{D}$ to $3 \mathrm{D}$ mapping technique discussed in Section 4.2.4.

Figure 27a shows the 2D model of the detonation. A $100 \mathrm{~g}$ spherical charge of PE4 was detonated in the $2 \mathrm{D}$ environment where the size of the air mesh was $0.3 \mathrm{~mm}$ which resulted in a total of 59,192 2D axisymmetric quadrilateral elements. Figure 28a shows the 3D model where the blast wave is allowed to interact with the fixed steel target acceptor plate used in the experiment. As the experiment was conducted and controlled in a laboratory setting, accurately achieving a fully fixed support condition was possible. Therefore, to reduce number of elements, the target acceptor plate was modelled as a rigid steel plate in the 3D model using shell elements with a $1 \mathrm{~mm}$ mesh size, resulting in a total of 62,500 elements. This assumption was considered acceptable as the response 
of the plate was not the focus of the experiment, nor was it the focus in this section of the validation. The steel plate material was modelled using MAT_PIECEWISE_LINEAR_PLASTICITY. The air in the 3D model was made up of solid elements and had a mesh size of $2 \mathrm{~mm}$, which resulted in a total of 984,375 elements. Pressure-time history was collected at the centre of the plate (to compare with the normally reflected pressures) as well as $100 \mathrm{~mm}$ from the centre (to compare with the obliquely reflected pressures).

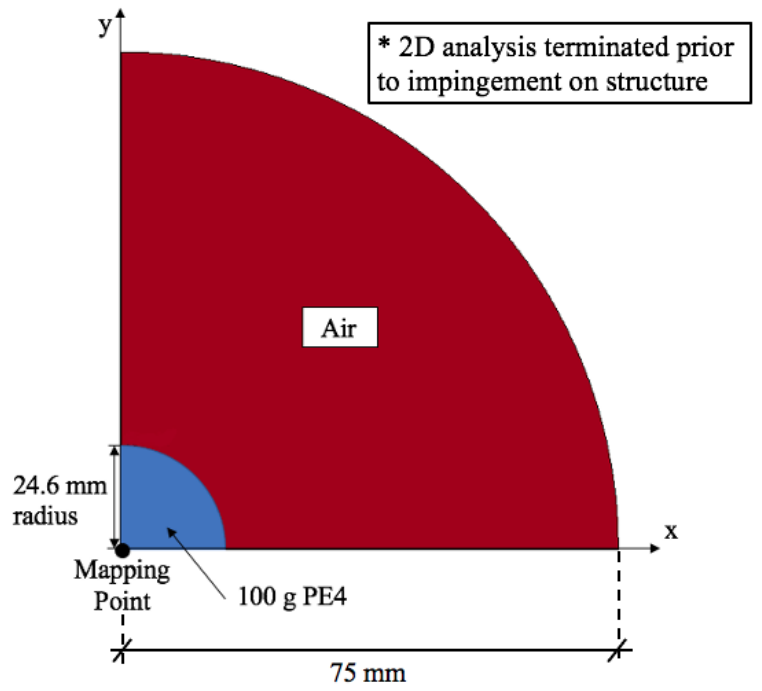

(a)

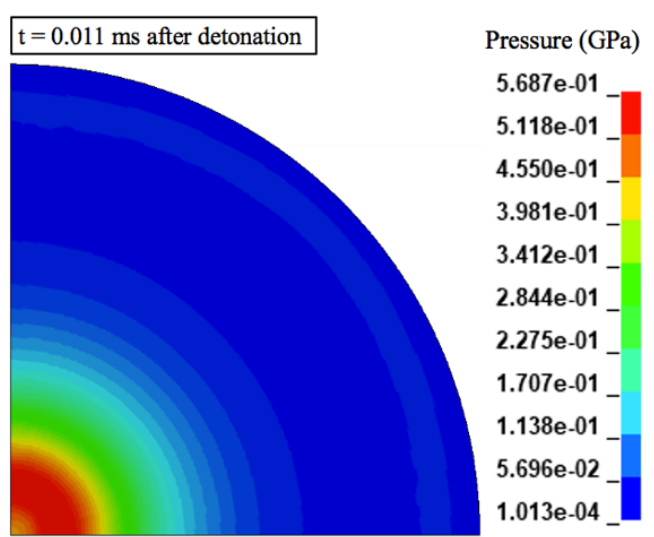

(b)

Figure 27: (a) 2D FE model of Rigby et al. (2015) (b) Propagation of blast pressures just prior to mapping.

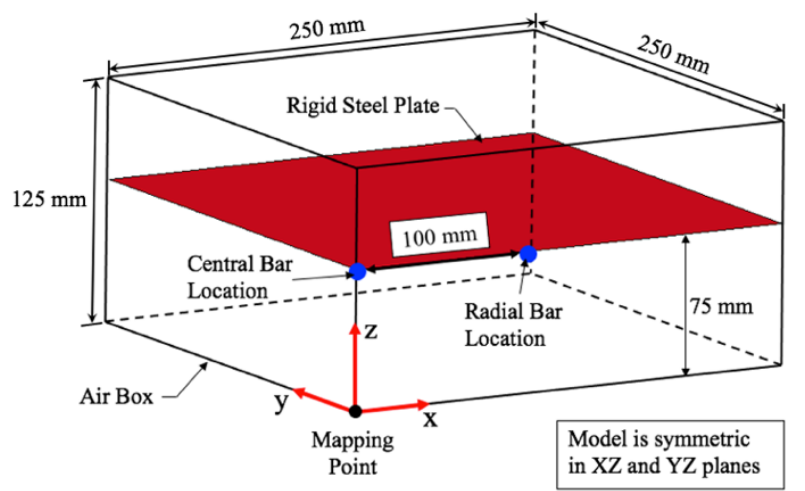

(a)

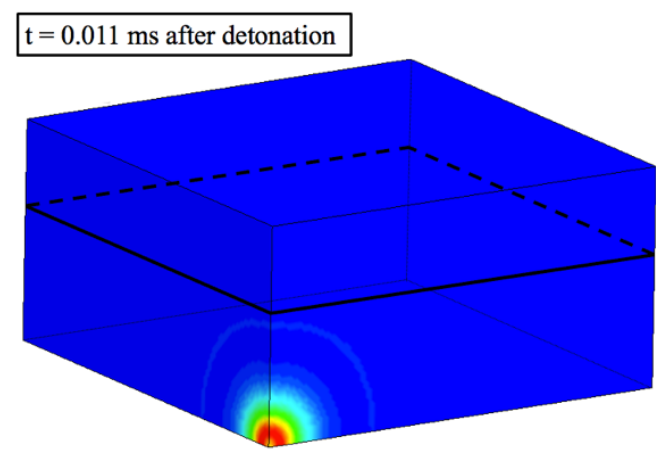

Pressure (Gpa) $5.670 \mathrm{e}-01$ 5.103e- 01 $4.536 \mathrm{e}-01$ $3.969 \mathrm{e}-01$ $3.402 \mathrm{e}-01$ $2.835 \mathrm{e}-01$ $2.268 \mathrm{e}-01$ $1.701 \mathrm{e}-01$ 1.134e-01 $5.670 \mathrm{e}-02$ $-0.000 \mathrm{e}+00$

Figure 28: (a) 3D FE model of Rigby et al. (2015) (b) Propagation of blast pressures just after mapping. 


\subsubsection{Results and Discussion}

Six test results were reported by Rigby et al. (2015) for redundancy and confidence in the measurements and to evaluate repeatability of the tests. Figure 29 to Figure 38 present the raw pressure data reported from the tests as well as the FE model's predictions of pressure. For example, Figure 29 plots the pressure-time histories collected by the central bar (Bar 5) for all six tests. Figure 30 plots the maximum and minimum pressures taken from Figure 29 demonstrating the variation in data obtained from similar tests. The FE model's prediction for this bar is also plotted and compared to the experimentally reported maximum and minimum pressure values.

Figure 31 to Figure 38 show similar comparisons for the radial bars in the experiment (Bars 1-4). These bars were all placed at a distance of $100 \mathrm{~mm}$ from the centre of the plate. Since the explosive charge shape was spherical, the spatial pressure distribution was also spherical, and these bars all collected the same pressures. The oscillations present in the raw data were a result of PochammerChree dispersion. The authors used perimeter-mounted semiconductor strain gauges which were located $250 \mathrm{~mm}$ from the loaded face of the Hopkinson pressure bars. The axial stress pulse which propagates along the bar and over the gauge location will cause a change in strain at the perimeter and the gauges can measure the temporal variation of the blast pressure. However, as the stress pulse propagates along the Hopkinson bar, each Fourier frequency component travels at a different velocity, causing some frequencies to fall out of sync, resulting in oscillations in the data (Rigby et al., 2015).

From the following figures, it is clear that the FE model can consistently predict a pressure-time history that falls within the maximum and minimum pressures collected in the experiment. The slow rise to peak pressure in the raw test data is a result of a time lag due to the transit time of the stress pulse from the front face of the Hopkinson bar to the strain gauge. This is more clearly 
observed when the FE model prediction is plotted against the experimental pressure-time history responses, such as in Figure 30. The authors indicated that variation of collected pressures between similar tests could be due to incomplete detonation of the explosive or a charge placement that was slightly off centre (Rigby et al., 2015). These factors would result in marginally lower recorded pressures. Furthermore, in the experiment the first three tests used non-electric detonators while tests four to six used electric detonators which could also have contributed to the variation in pressure data.

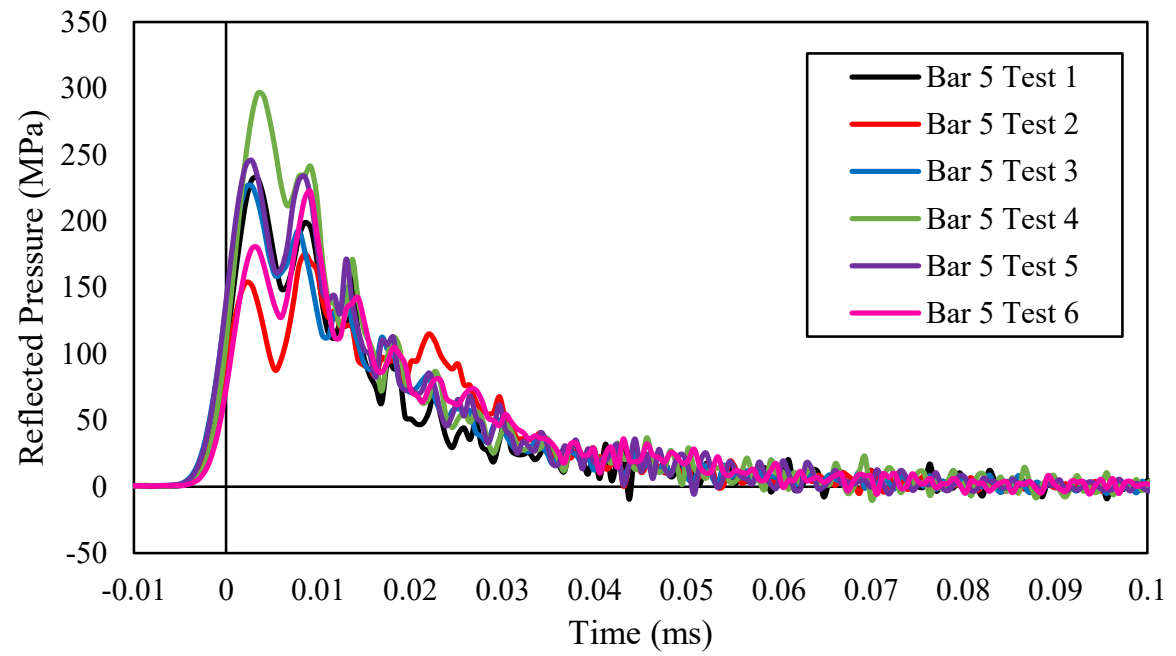

Figure 29: Raw experimental pressure data for the central bar (Bar 5) (Rigby et al., 2015).

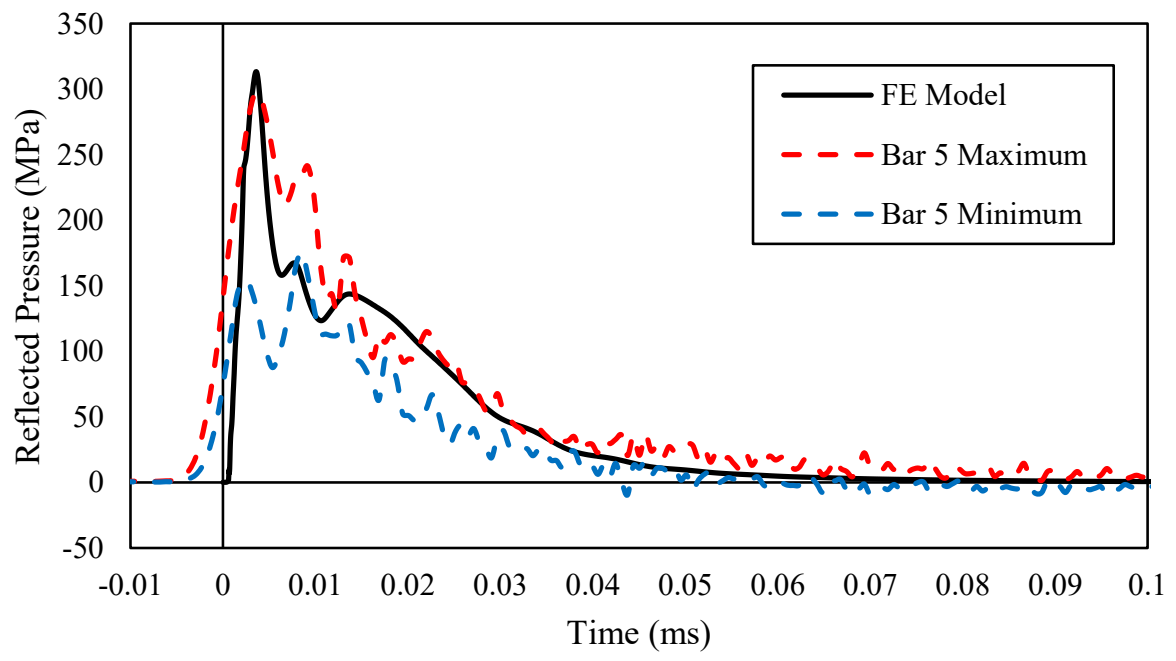

Figure 30: Comparison of FE model prediction for central bar (Bar 5) against maximum and minimum experimental results. 


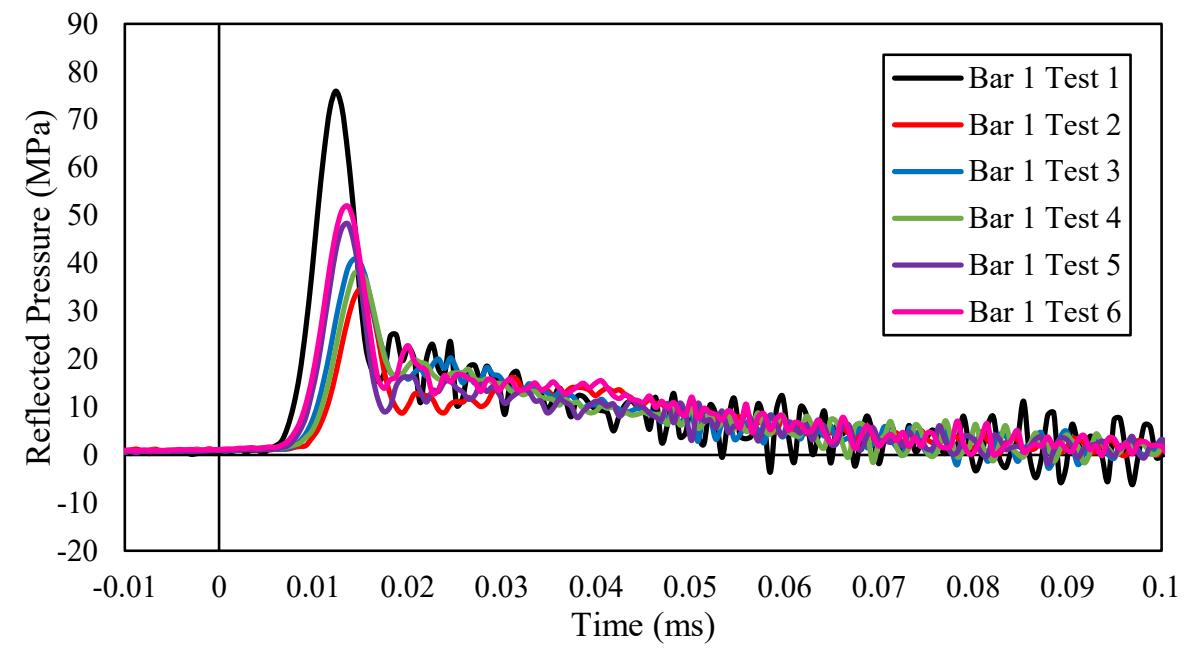

Figure 31: Raw experimental pressure data for a radial bar (Bar 1) (Rigby et al., 2015).

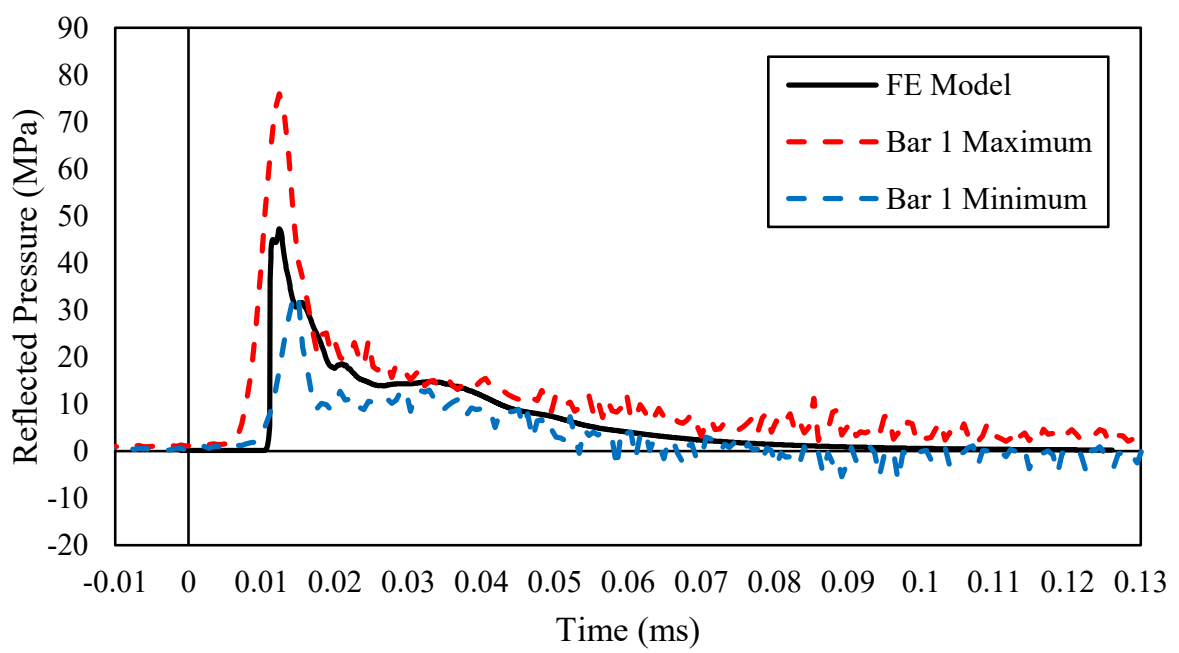

Figure 32: Comparison of FE model prediction for radial bar against maximum and minimum experimental results for Bar 1.

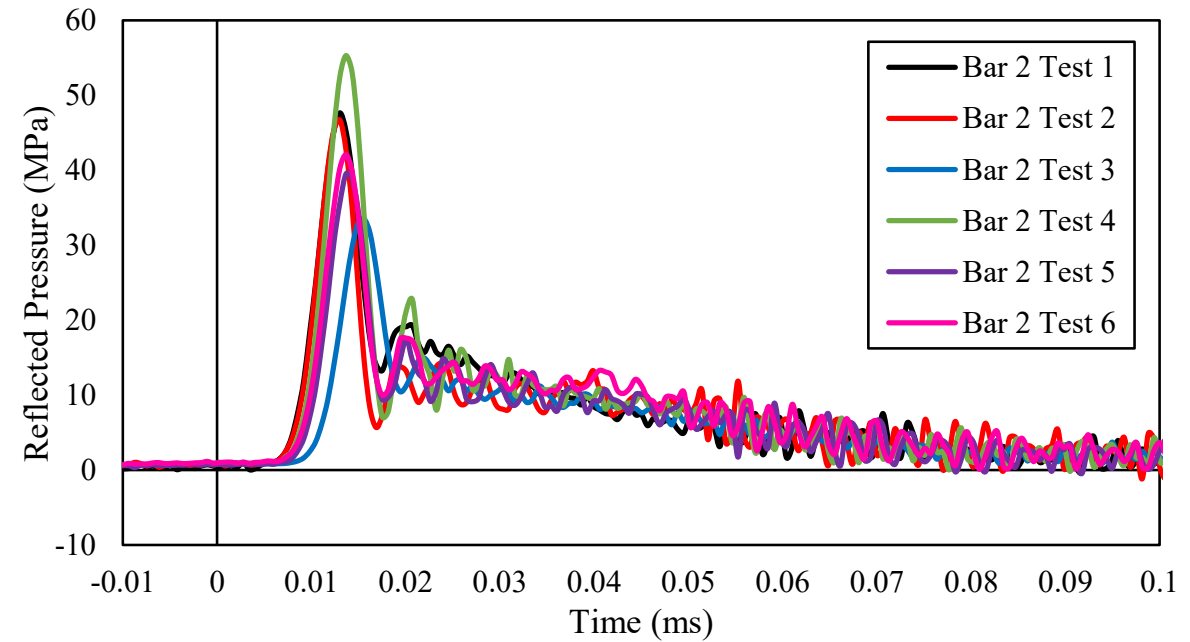

Figure 33: Raw experimental pressure data for a radial bar (Bar 2) (Rigby et al., 2015). 


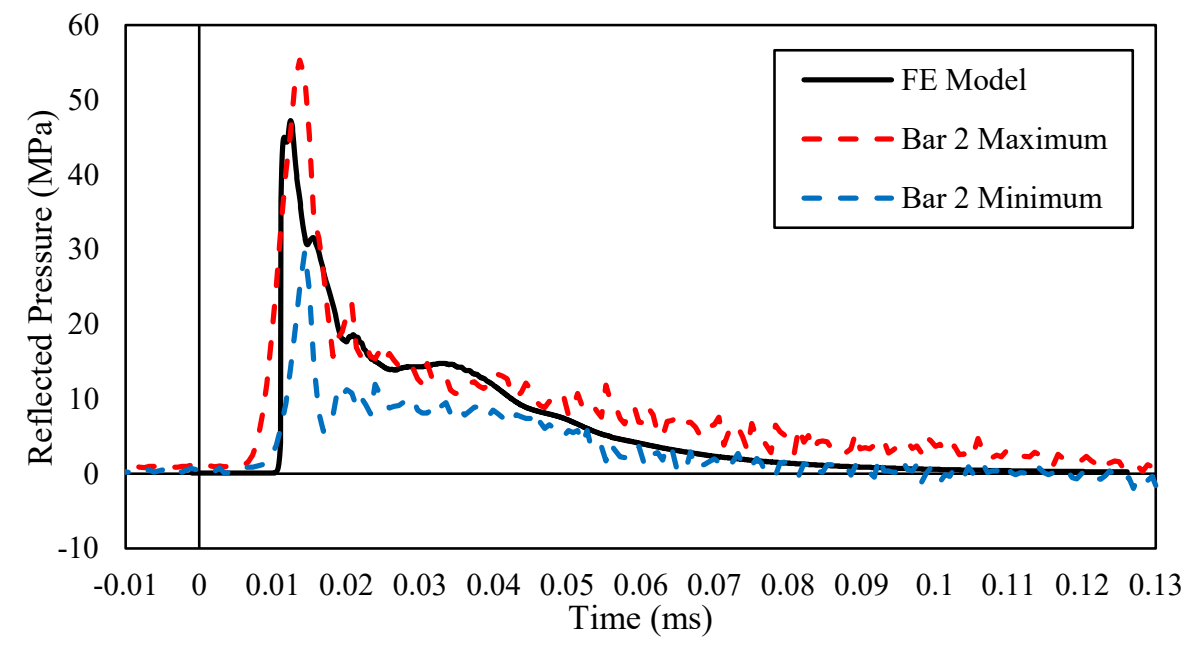

Figure 34: Comparison of FE model prediction for radial bar against maximum and minimum experimental results for Bar 2.

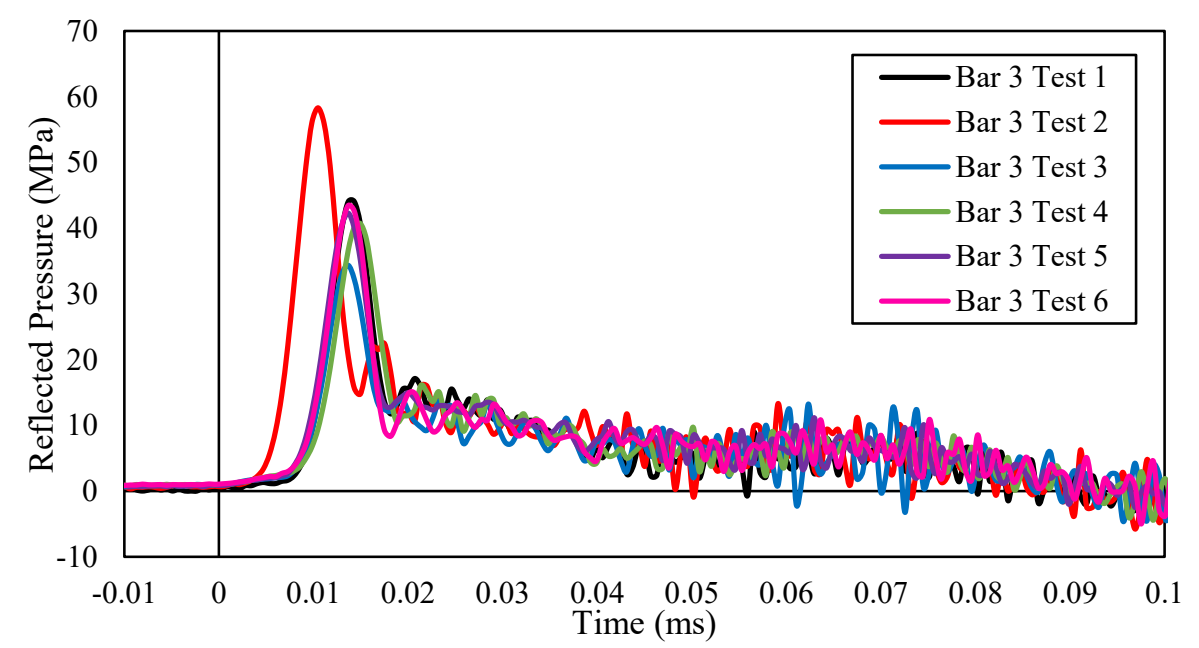

Figure 35: Raw experimental pressure data for a radial bar (Bar 3) (Rigby et al., 2015).

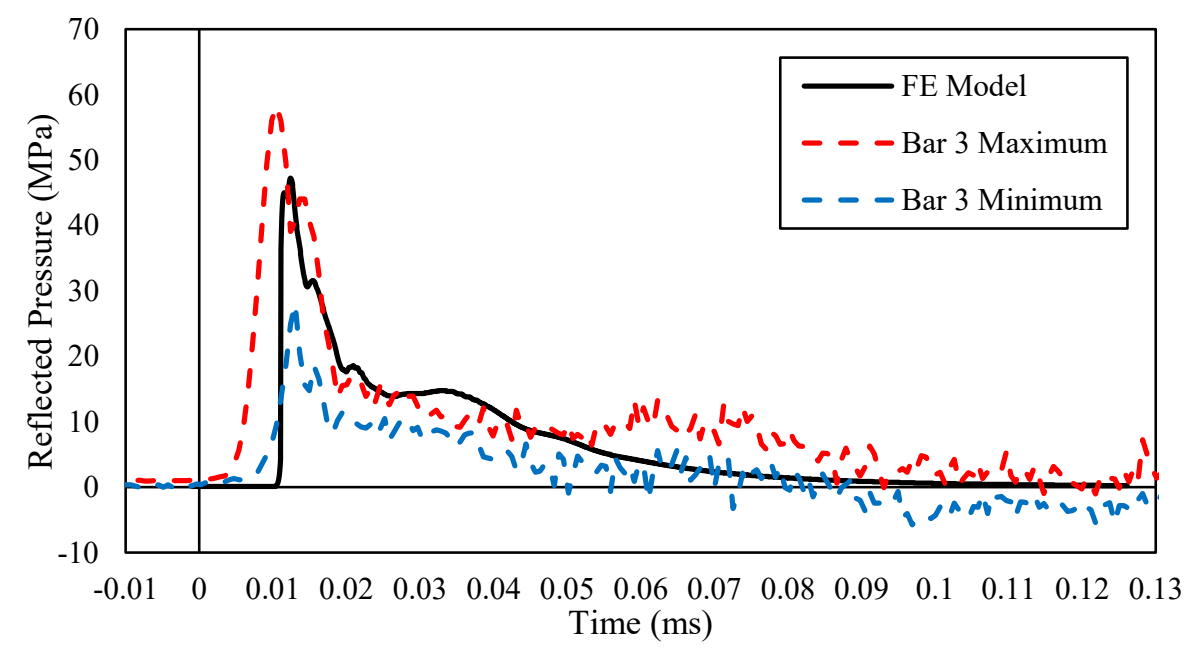

Figure 36: Comparison of FE model prediction for radial bar against maximum and minimum experimental results for Bar 3 . 


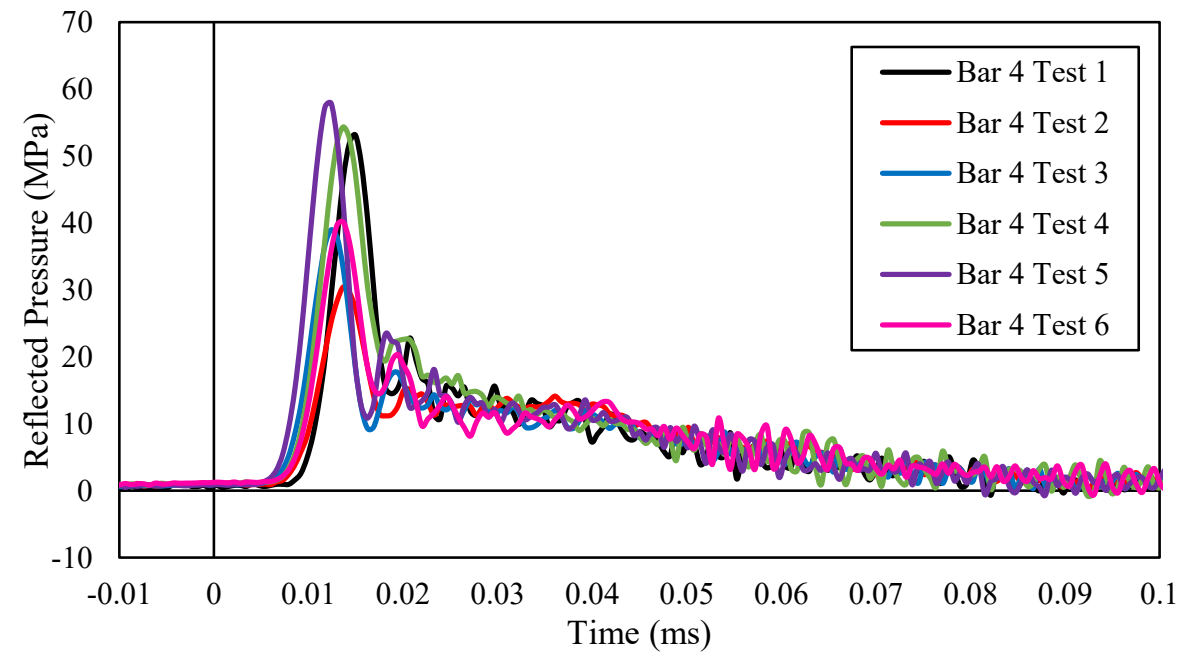

Figure 37: Raw experimental pressure data for a radial bar (Bar 4) (Rigby et al., 2015).

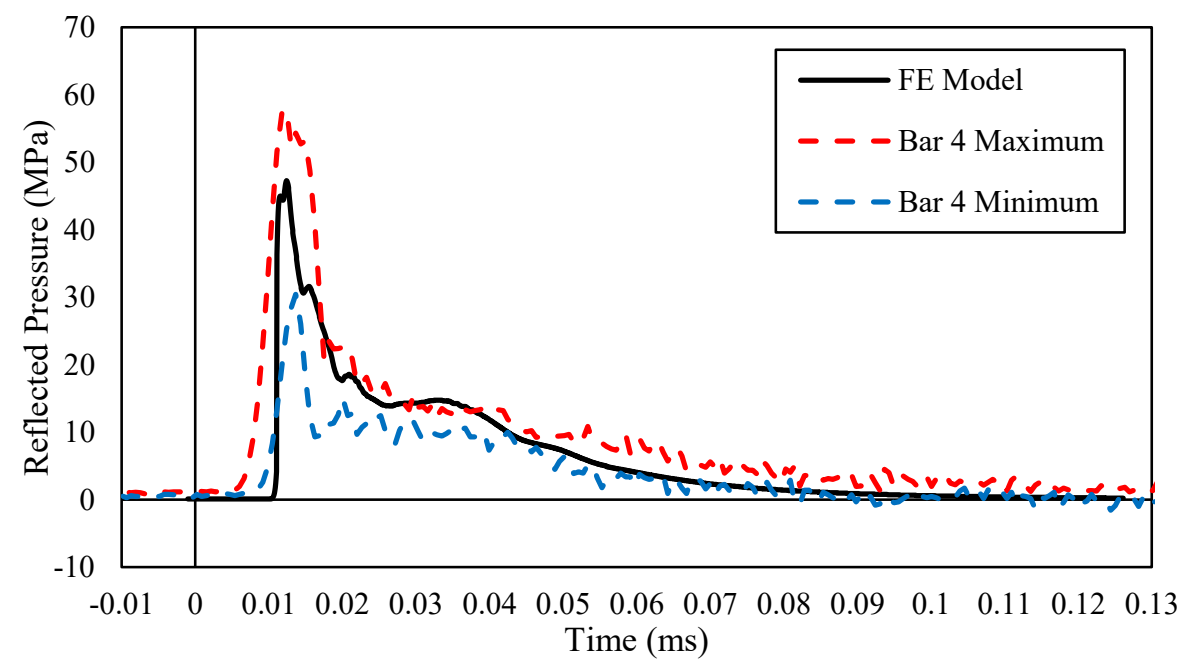

Figure 38: Comparison of FE model prediction for radial bar against maximum and minimum experimental results for Bar 4.

Figure 39 and Figure 40 compare the analytical and experimental pressure- and impulse-time histories for the centrally located bar. The experimental data plotted in these figures were collected by Bar 5 from the fifth test. These curves have been corrected by the authors, removing a large portion of the oscillations seen in the raw data due to the Pochammer-Chree dispersion. This dispersion correction also addressed the slow rise to the peak pressure observed in the raw experimental data. The almost instantaneous rise to peak pressure seen in the FE model's prediction, is now also observed in the corrected experimental pressure. 
Labelled as Bar 5 in Figure 26b, this bar collected normally reflected blast pressures. Figure 39 shows a discrepancy between the peak blast pressure predicted in the FE model and that recorded in the experiment. The FE model overestimates the peak pressure by approximately $35 \%$. This overestimation could result from the complex interaction of the shock front and air/explosive interface that occurs in near-field explosions. The turbulent mixing of air and detonation products at the interface may actually lessen the magnitude of the shock front. The FE model may be simplifying this interaction as well as the clearing process of the blast wave, thereby overestimating the peak pressure and somewhat idealizing the pressure profile (Rigby et al., 2015). The discrepancy in peak pressure could also be due to an incomplete charge detonation or charge placement that was slightly off-centre. As mentioned previously, these two factors could result in lower pressures.

However, as shown in Figure 40, the FE model can capture the impulse-time history very well with minor deviations from the experimental data. The impulse is calculated as the area under the pressure-time history. Therefore, any deviations in the pressure profile would also affect the prediction of the impulse.

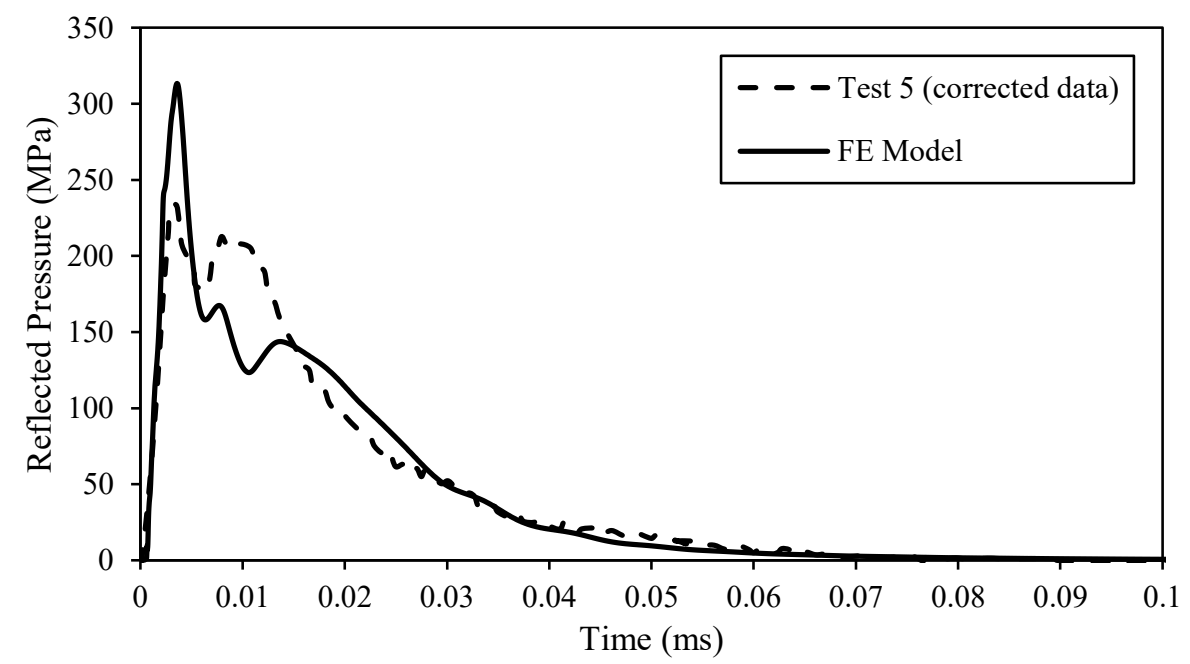

Figure 39: Comparison of analytical and experimental pressure-time history for the central bar (z $\left.=0.15 \mathrm{~m} / \mathrm{kg}^{1 / 3}\right)$ 


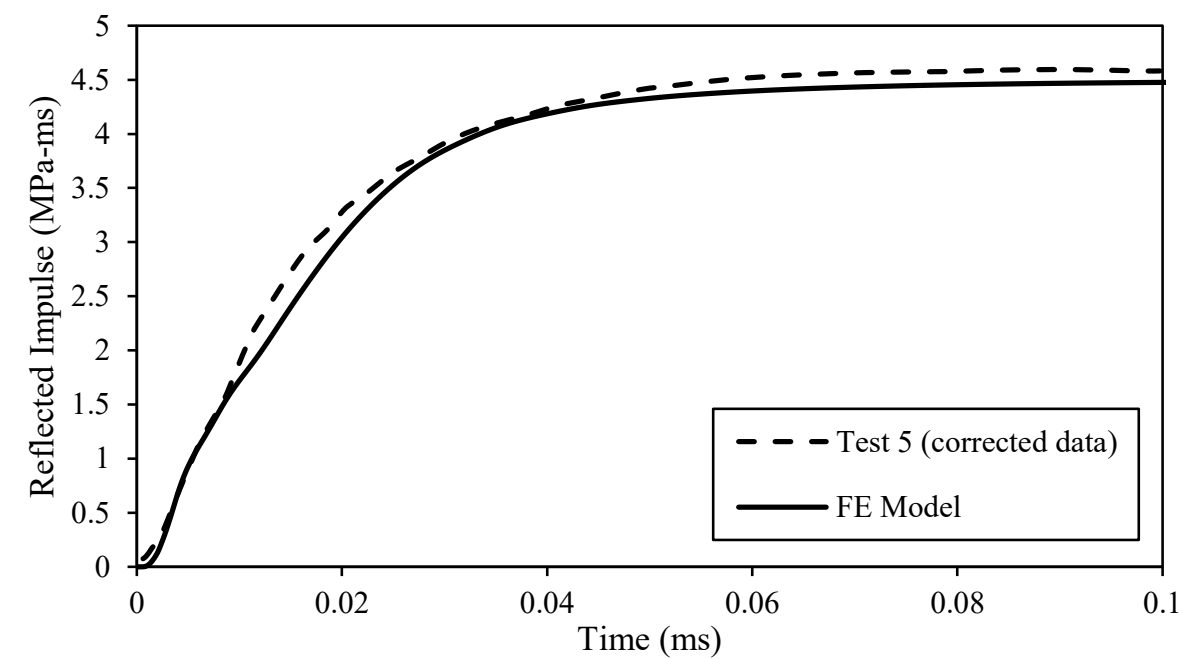

Figure 40: Comparison of analytical and experimental impulse-time history for the central bar (z $=0.15 \mathrm{~m} / \mathrm{kg}^{1 / 3}$ )

Figure 41 and Figure 42 compare the analytical and experimental pressure- and impulse-time histories for the radial bars. Labelled as Bars 1-4 in Figure 26b, these bars collected obliquely reflected blast pressures. The experimental data plotted in these figures were collected by Bar 4 from the fifth test with the dispersion correction applied.

The pressure-time history for the radial bars seems to be better captured by the FE model than for the central bar. However, as observed in Figure 41, the peak pressure is again still slightly overestimated by approximately $17.5 \%$. As discussed earlier, this is perhaps due to the mixing of the air and detonation products at the interface. The effect of this same phenomenon would also be lessened further from the centre of the slab as the blast wave clears, which is perhaps why the FE model is able to better capture the radial pressures. Similar to the central bar, the FE model can predict the impulse quite well, showing minor deviations in the same places where the FE pressure profile deviates from the experiment.

One final factor that may be improving the accuracy of the radial bar prediction may be related to leakage. Leakage of fluids (explosive and air) into the Lagrangian elements can result in inaccuracies. While leakage controls are included in the FE model, some degree of leakage can 
still occur. The most leakage tends to occur in the region where the fluids first interact with the Lagrangian structure (in this case, the centre of the plate). Leakage tends to diminish, and fluidstructure coupling becomes more stable as the blast wave propagates across the structure. A small amount of leakage is typical and acceptable but may be a contributing factor to the improved accuracy of the computed pressure and impulse values for the radial bar compared to the central bar results.

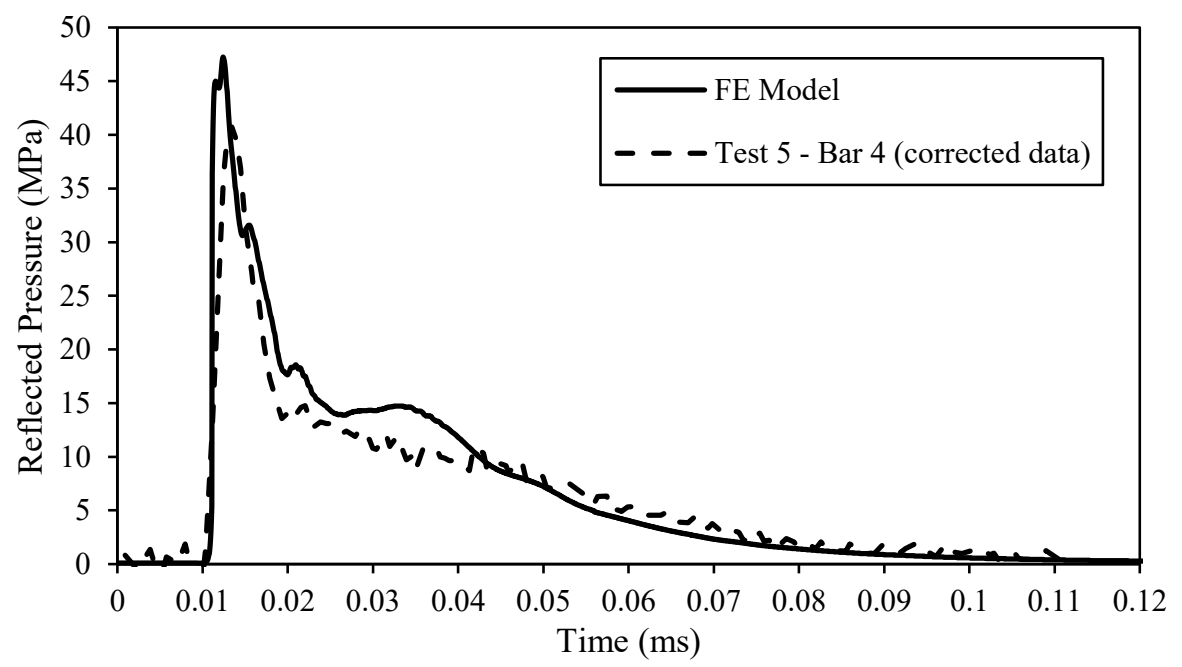

Figure 41: Comparison of analytical and experimental pressure-time history for the radial bar (z $=0.25 \mathrm{~m} / \mathrm{kg}^{1 / 3}$ and $\alpha=53^{\circ}$ )

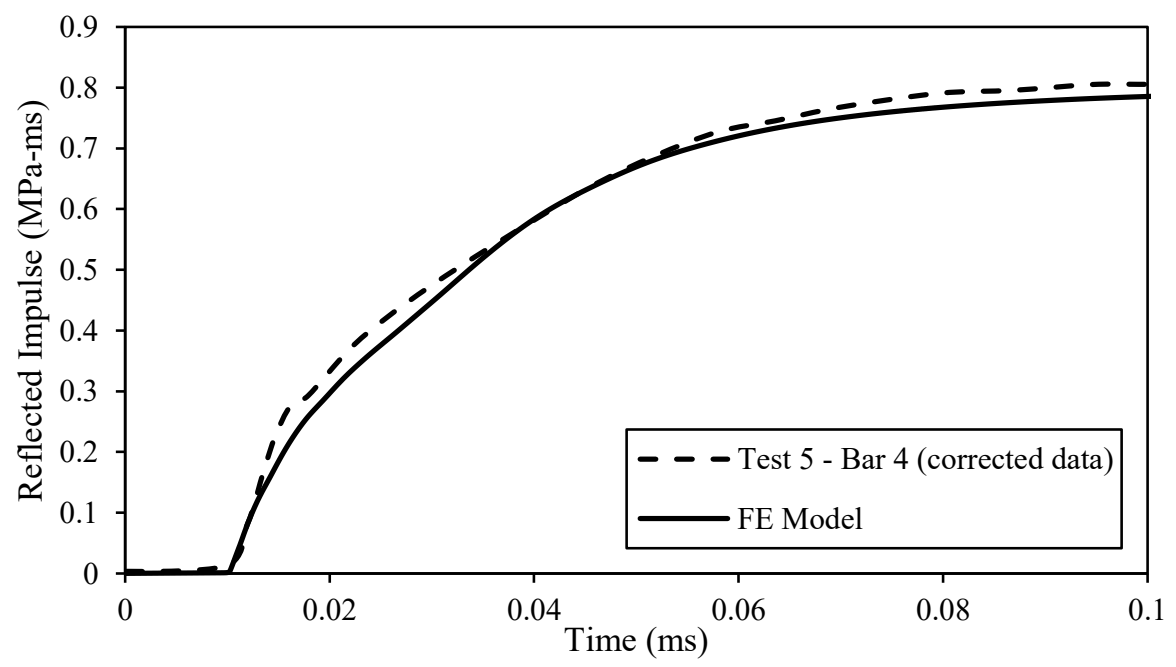

Figure 42: Comparison of analytical and experimental impulse-time history for the radial bar ( $\mathrm{z}=$ $0.25 \mathrm{~m} / \mathrm{kg}^{1 / 3}$ and $\alpha=53^{\circ}$ ) 
Table 8 lists the experimental peak reflected pressures of all five bars for all six tests. It also reports the average pressure of each bar based on all six tests in comparison to the peak pressures predicted by the FE model. It can be seen that there is some variation between tests for the experimentally recorded pressures which is due to the complexity involved in measuring blast pressures of nearfield explosions. The FE model is able to predict the average pressures of the radial bars (Bars 14) with good accuracy but overestimates the peak pressure of the central bar (Bar 5). As previously mentioned, this is potentially due to the fact that the FE model idealizes the mixing of the air and detonation products at the interface. However, as previously shown, the FE model is able to capture the pressure decay recorded by this bar as well as its reflected impulse. Most importantly, the FE model was able to consistently predict the blast pressure profile within the range of the maximum and minimum pressures reported in the study.

Table 8: Comparison of peak and average reflected pressures of all bars for all tests to FE model prediction.

\section{Peak Reflected Pressure (MPa)}

\begin{tabular}{c|ccccc}
\multicolumn{1}{c}{} & Bar 1 & Bar 2 & Bar 3 & Bar 4 & Bar 5 \\
\hline Test 1 & 76.0 & 47.7 & 44.0 & 53.1 & 232.1 \\
Test 2 & 34.7 & 46.7 & 58.3 & 30.4 & 175.3 \\
Test 3 & 40.9 & 33.5 & 34.4 & 39.0 & 226.6 \\
Test 4 & 38.2 & 55.3 & 40.7 & 54.3 & 296.3 \\
Test 5 & 48.3 & 39.5 & 42.3 & 57.9 & 245.7 \\
Test 6 & 51.9 & 42.1 & 43.5 & 40.1 & 221.9 \\
\hline $\begin{array}{c}\text { Average for } \\
\text { all tests }\end{array}$ & 48.3 & 44.1 & 43.9 & 45.8 & 233.0 \\
\hline FE Model & 47.2 & 47.2 & 47.2 & 47.2 & 313.3
\end{tabular}

Because the tests were conducted in a laboratory setting, the authors had more control over the experimental setup and support conditions (which is not the case in field tests). With fewer uncertainties, the experiment was easier to simulate using FE modelling. Furthermore, Hopkinson pressure bars have been used for many years as a robust transient force transducer, providing a 
reliable pressure measurement method (Tyas \& Watson, 2001). Also, as previously mentioned, the margin of error in blast validation is higher than typically expected in engineering research. As a result, the level of accuracy obtained from the FE analyses is considered to be acceptable.

Based on the validation results, the variation of certain blast parameters across the plate is plotted. Figure 43 shows the time of arrival of the blast wave across the plate. The distance of $0 \mathrm{~mm}$ is located at the centre of the plate. Figure 44 shows the variation of peak pressure across the plate and Figure 45 shows the variation of impulse across the plate. For comparison, Figure 44 and Figure 45 also include the peak pressure and impulse recorded in the experiment. The experimental peak values plotted were taken from the corrected data shown in Figure 39 - Figure 42. However, the authors did not include exact arrival times in the experiment, so no comparison is made in Figure 43. These values are plotted at the centre of the plate and at $100 \mathrm{~mm}$ from the centre of the plate. These figures demonstrate the highly nonlinear variation of the blast wave as it interacts with a structure in the near-field. This supports the importance of the use of CFD in the modelling of near-field blast loading, particularly in scaled distances below $0.4 \mathrm{~m} / \mathrm{kg}^{1 / 3}$.

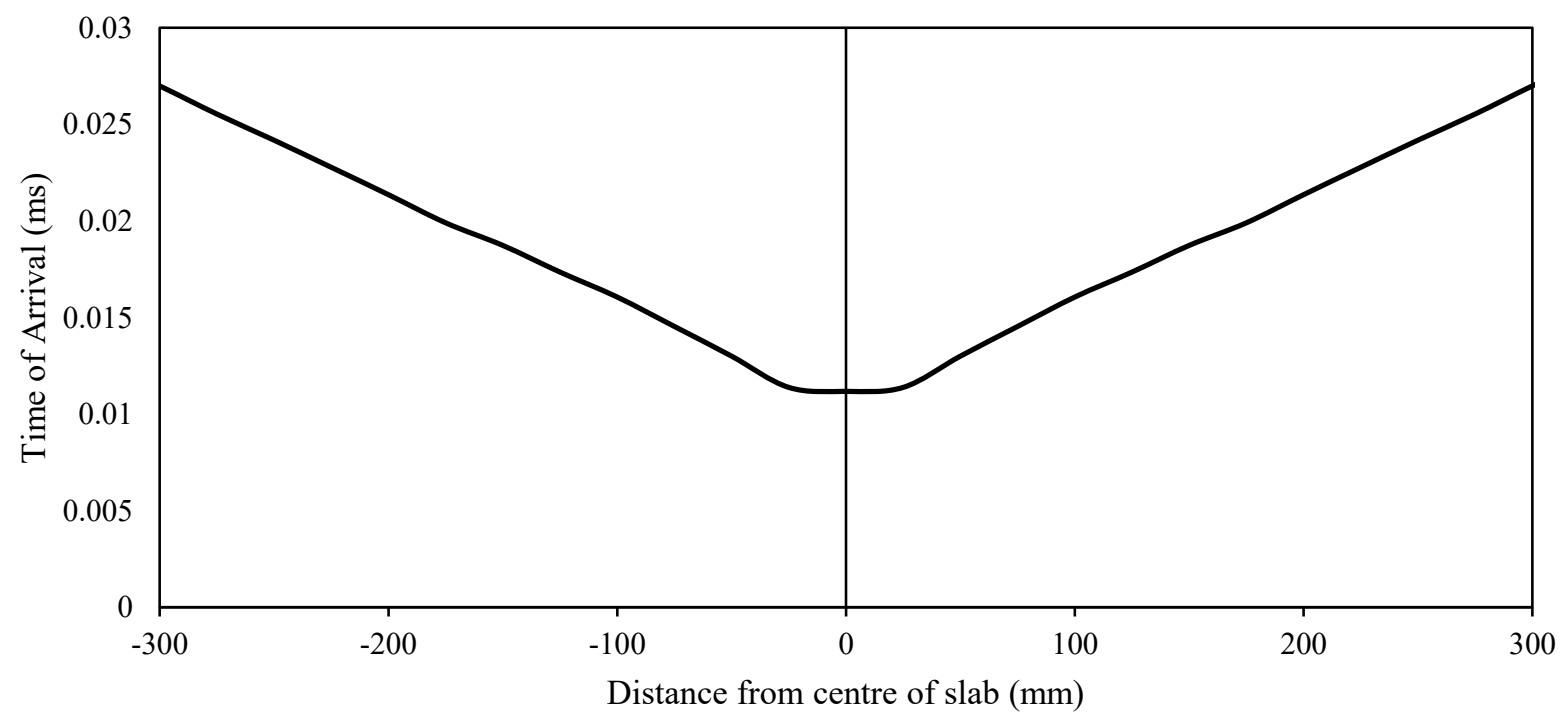

Figure 43: Time of arrival of the blast wave across the plate as predicted by the FE model. 


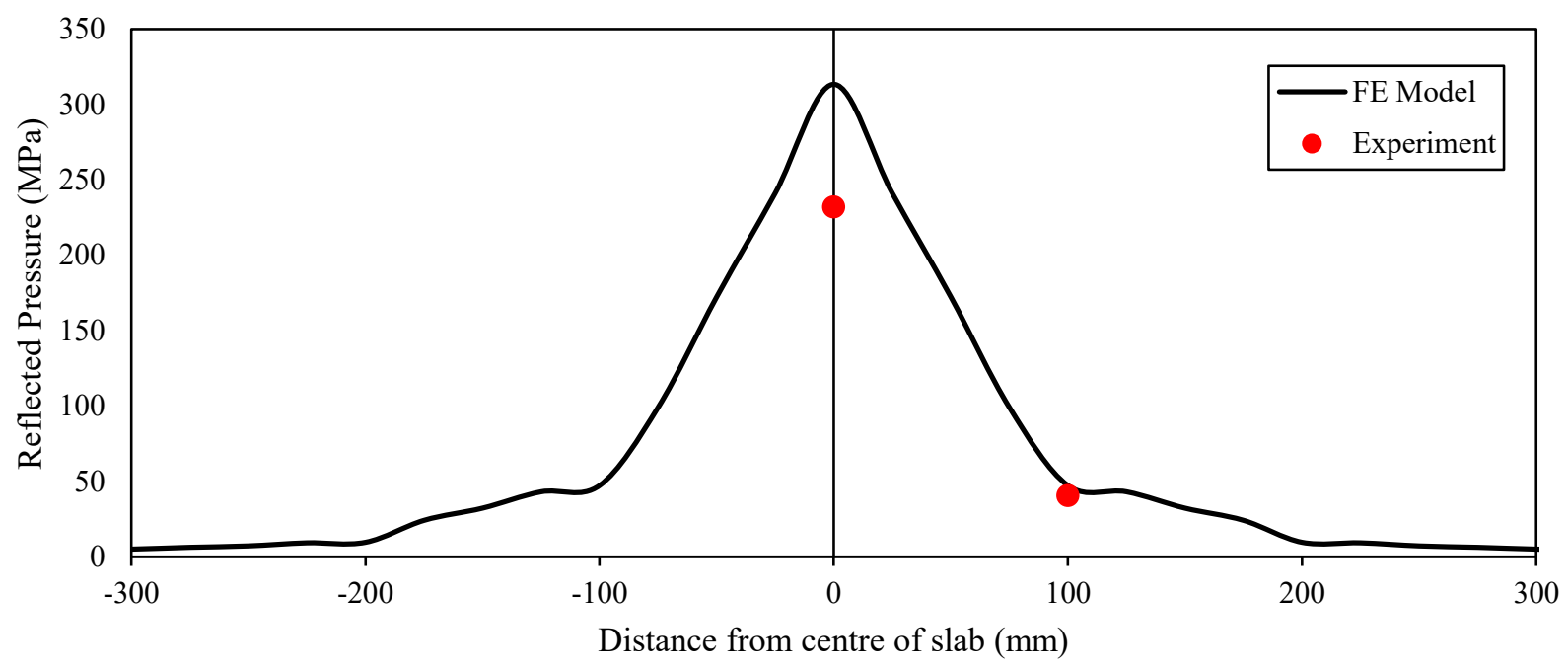

Figure 44: Comparison of peak pressure variation across the plate as predicted by the FE model with experimentally reported values.

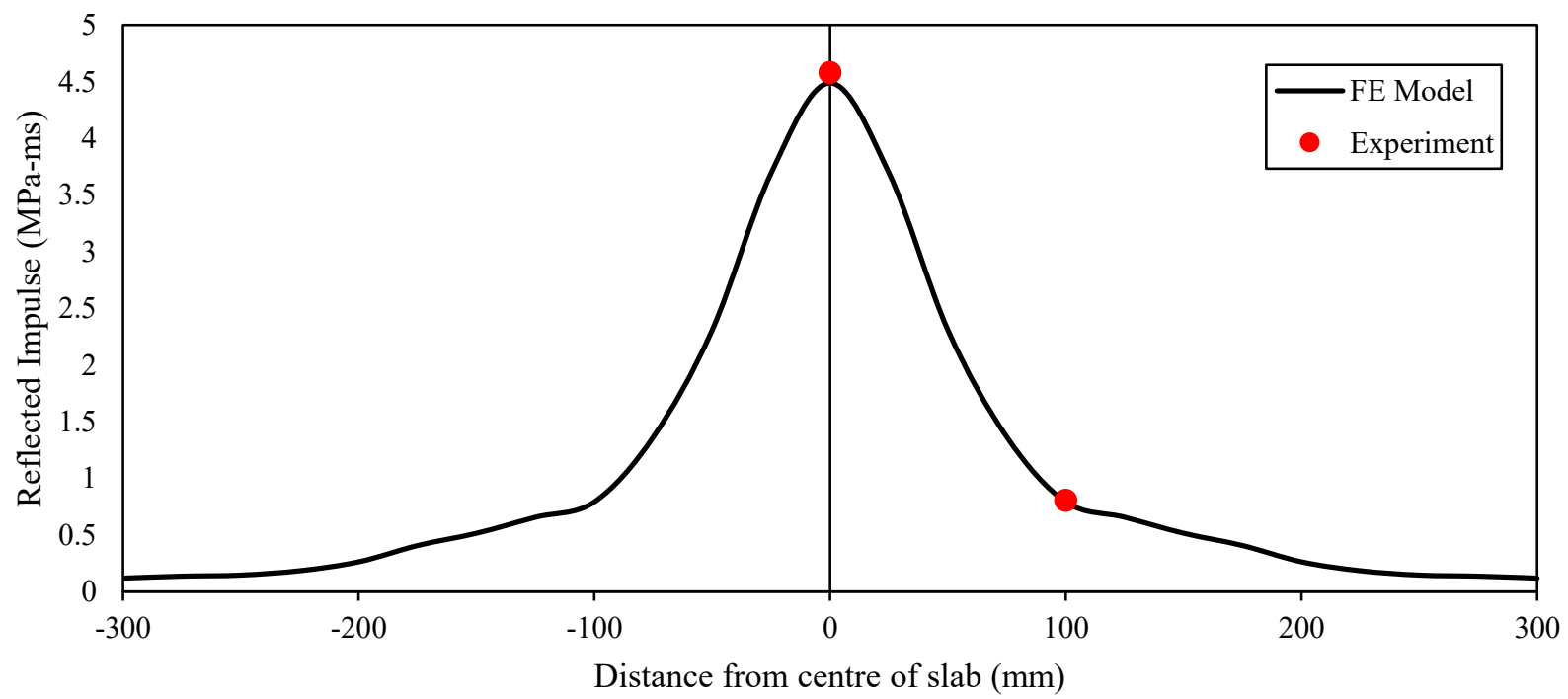

Figure 45: Comparison of impulse variation across the plate as predicted by the FE model with experimentally reported values. 


\subsubsection{Validation against Yang et al. (2019) Test}

\subsubsection{Experiment Details}

A second validation was conducted using the experimental results by Yang et al. (2019). The authors conducted a series of field explosion tests on crumbed rubber concrete (CRC) slabs with steel reinforcement. Three normal strength $\mathrm{RC}$ slabs (with no rubber particles) were included as control test specimens. The slabs were subjected to close-in blast loads, and the slab deflection was measured at midspan using a linear variable differential transformer (LVDT) transducer. The pressure-time history was also collected $50 \mathrm{~mm}$ from the centre of the slab using a pressure transducer and was reported in the study. The test setup and instrumentation are shown in Figure 46.

As shown in Figure 46a, a spherical charge composed of ANFO was suspended above the centre of simply supported slab. The authors tested three loading cases all within the near-field in order to analyze different failure modes. The near-field pressure data collected from slab NC-3 was selected for validation, as the slab's response to the loading was also used in the second phase of the FE model validation. Slab NC-3 was subjected to $5.6 \mathrm{~kg}$ of ANFO at a distance of $500 \mathrm{~mm}$, resulting in a scaled distance of $\mathrm{z}=0.282 \mathrm{~m} / \mathrm{kg}^{1 / 3}$.

Figure 46c shows the dimensions of the slab as well as the layout of the reinforcement. The concrete had a uniaxial compressive strength of 34.91 MPa and the reinforcing steel had a yield strength of $235 \mathrm{MPa}$. Young's modulus of the steel reinforcement was reported as $210 \mathrm{GPa}$. Reinforcing bars of $8 \mathrm{~mm}$ diameter were used in compression and tension with a spacing of 200 mm. The slab was simply supported using steel supports, as shown in Figure 46a, and the displacement of the slab was collected at midspan $50 \mathrm{~mm}$ from the centre. 


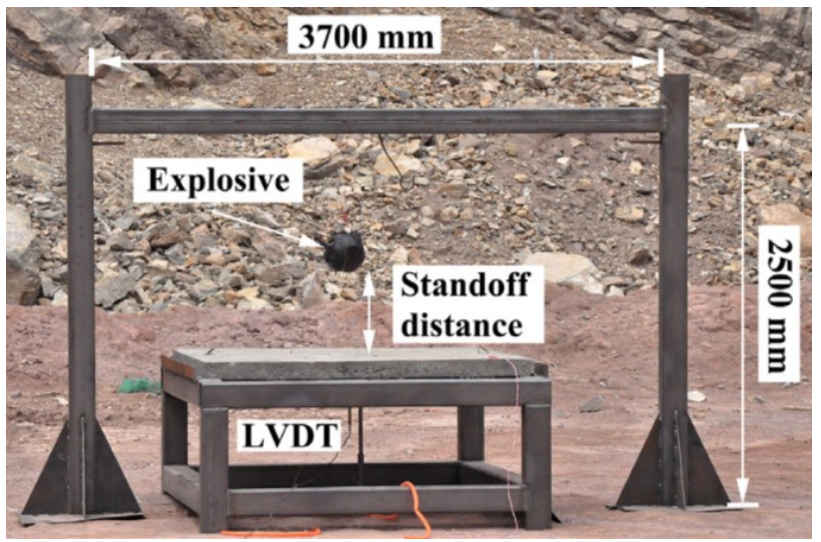

(a)

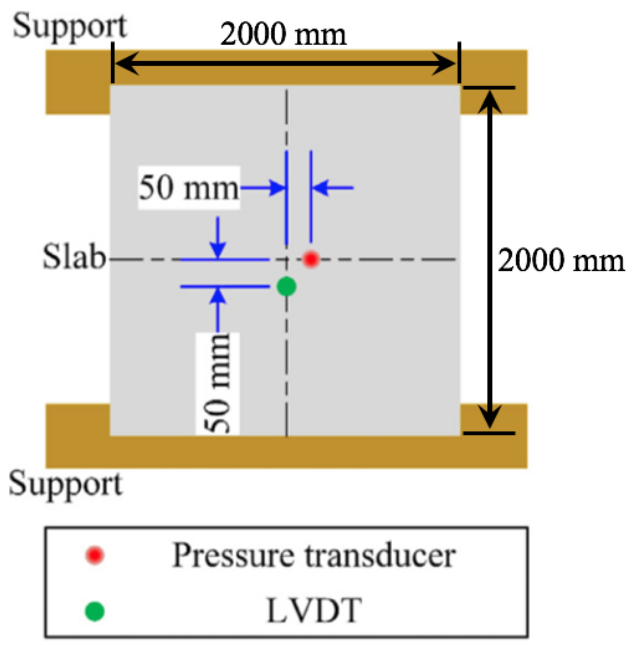

(b)

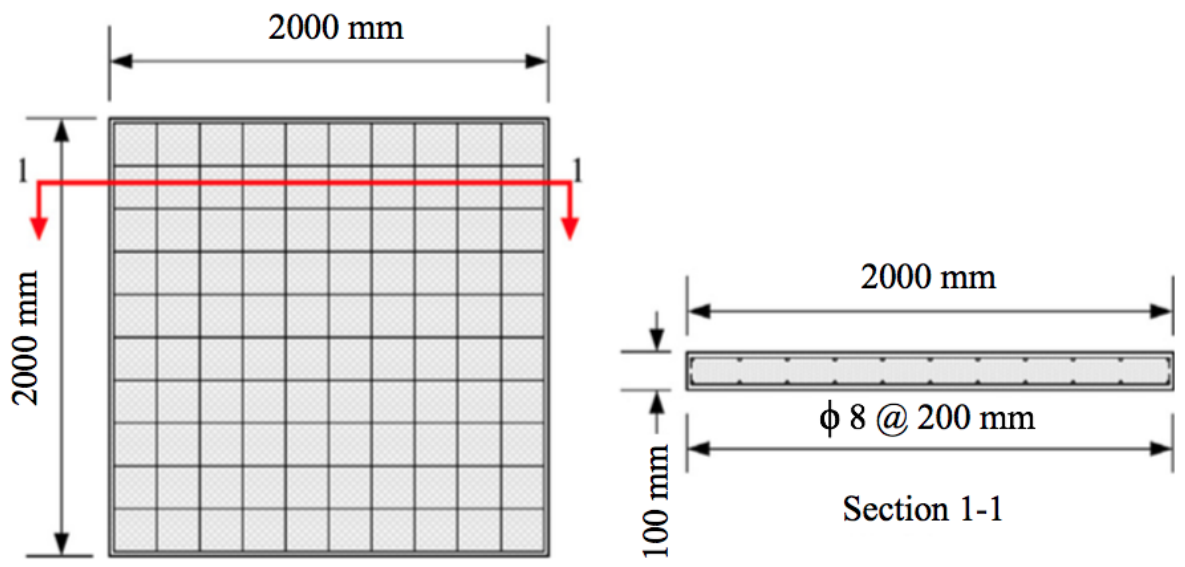

(c)

Figure 46: Field-testing system used by Yang et al. (2019): (a) test setup and (b) instrumentation details (c) slab dimensions and reinforcement configuration.

\subsubsection{FE Model}

The blast wave propagation was modelled in LS-DYNA using the $2 \mathrm{D}$ to $3 \mathrm{D}$ mapping technique described in Section 4.2.4. Taking advantage of the symmetry, only a quarter of the air, explosion, and structure were modelled. As shown in Figure 47a, the $5.6 \mathrm{~kg}$ of ANFO was detonated from a spherical charge in the 2D environment using a quarter-circular shell to model the air, which used 2D axisymmetric quadrilateral elements with a mesh size of $2 \mathrm{~mm}$, resulting in a total of 93,480 elements. The blast pressures were then mapped into the 3D model, shown in Figure 48b, where the blast wave was allowed to interact with the slab. It should be noted that the slab is shown 
upside down with blast loadings being applied from below. The air in the 3D model was made up of a $20 \mathrm{~mm}$ solid element mesh, resulting in a total of 650,000 elements. The blast pressures were collected $50 \mathrm{~mm}$ from the centre of the slab, just after reflection off the slab.

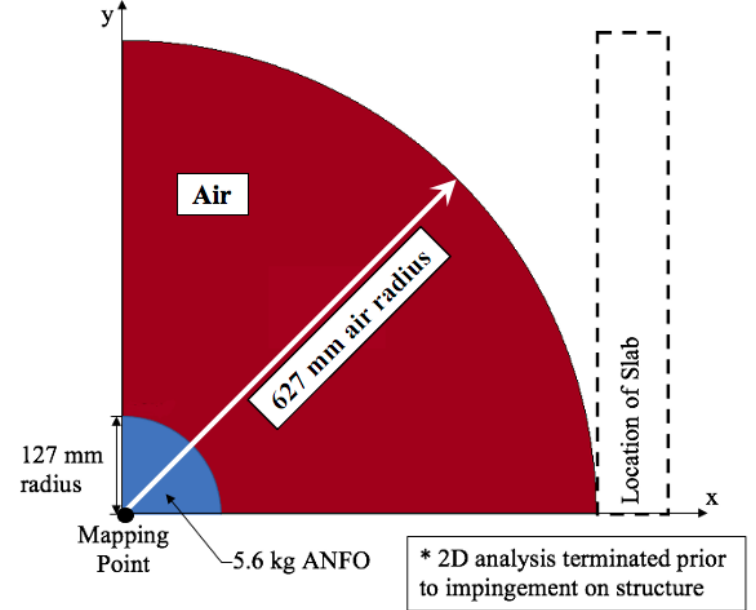

(a)

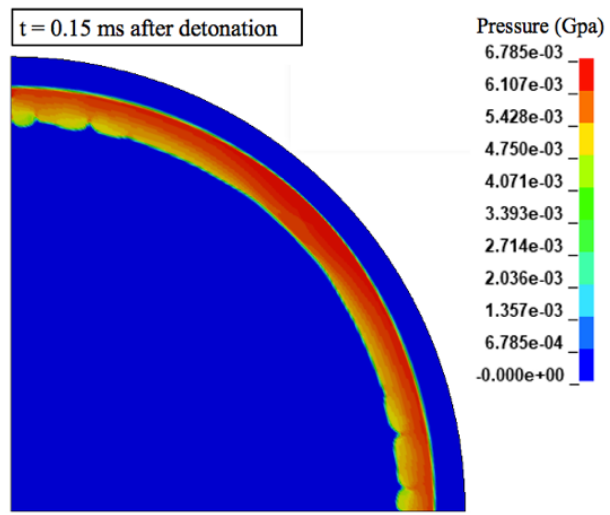

(b)

Figure 47: (a) 2D model of Yang et al. (2019) (b) Propagation of blast pressures just prior to mapping.

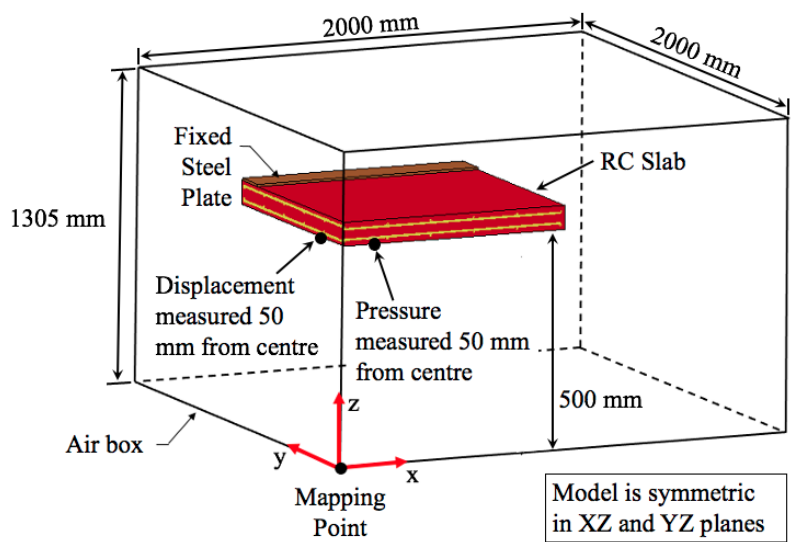

(a)

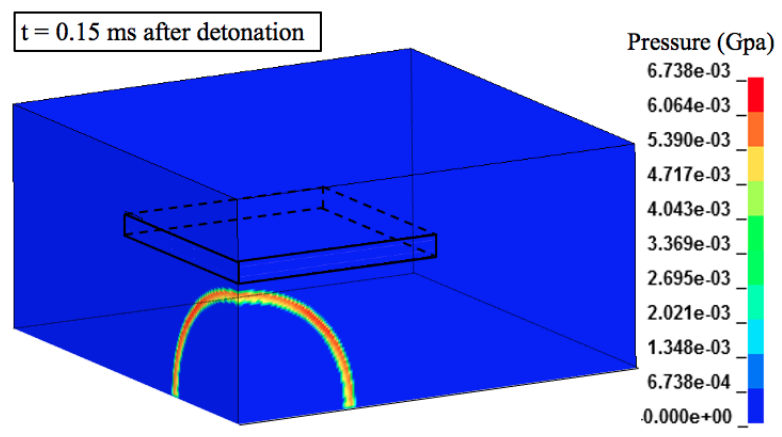

(b)

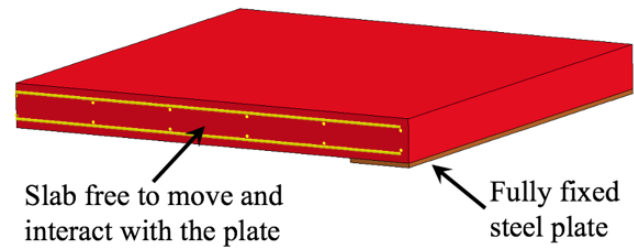

(c)

Figure 48: (a) 3D model of Yang et al. (2019) (b) Propagation of blast pressures just after mapping (c) Support condition of the slab (simply supported). 


\subsubsection{Results and Discussion}

Figure 49 compares the pressure-time history recorded in the FE model to the data collected in the experiment. The arrival time of the blast wave was not reported in the study, and the experimentally recorded blast pressure profile had been shifted so that the arrival time was zero. Therefore, the experimental pressure-time history begins before zero. The analytical results predicted by the FE model were also shifted accordingly. The FE model overestimates the peak pressure by approximately $22 \%$. However, it seems to be able to better estimate the impulse, as shown in Figure 50. The overestimation of the peak pressure could be due to the placement of the explosive relative to the pressure transducer which can affect the results significantly. As blast pressures in the near-field are highly dynamic and nonlinear, a slight change/error in the location of the charge or pressure transducer can have a significant effect on the results. Therefore, this type of human error could also be contributing to the discrepancy between the experimentally reported pressures and those predicted by the FE model. However, these results are considered to be acceptable for the verification of the FE model. Furthermore, the purpose of this second validation was to establish greater confidence in the FE model's ability to predict blast pressure and impulse in the near-field across independent studies. 


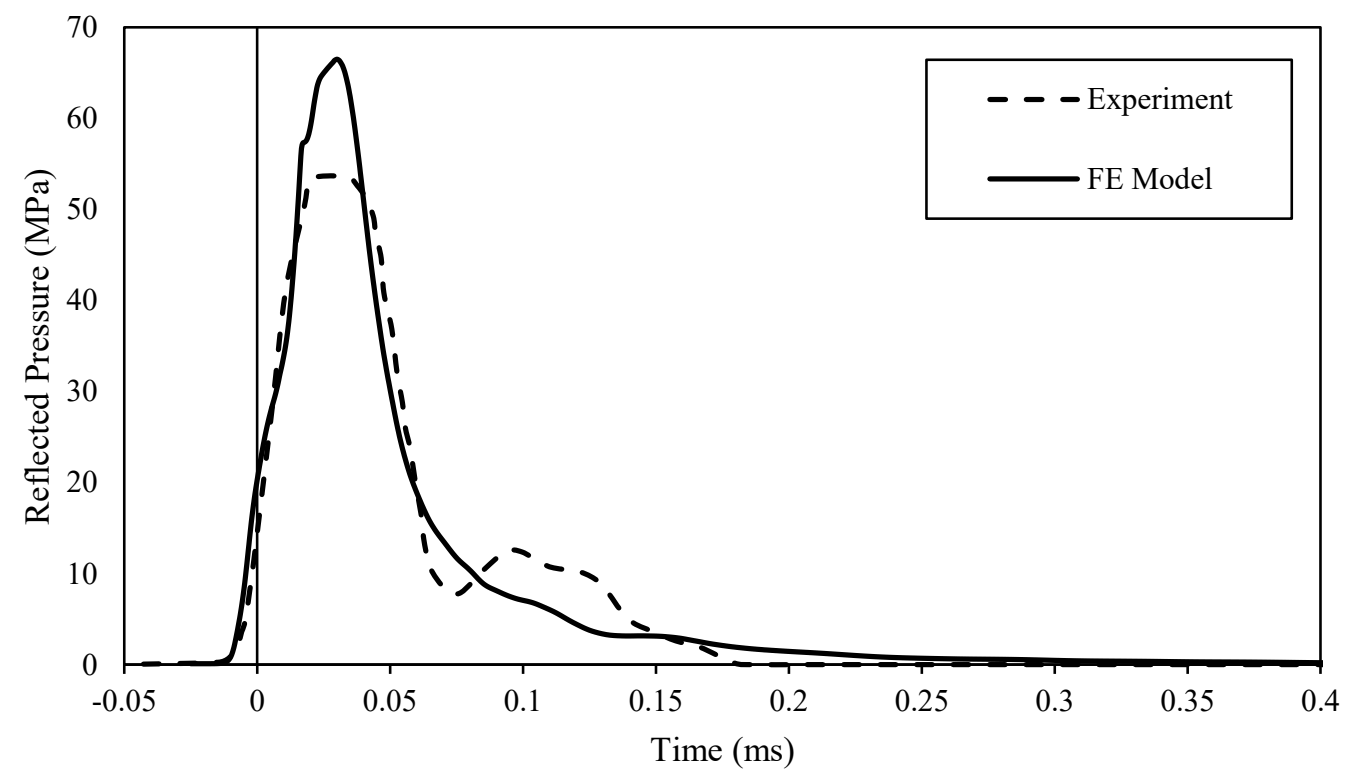

Figure 49: Comparison of analytical and experimental reflected pressure-time histories for $5.6 \mathrm{~kg}$ ANFO with a $500 \mathrm{~mm}$ stand-off distance $\left(\mathrm{z}=0.282 \mathrm{~m} / \mathrm{kg}^{1 / 3}\right)$.

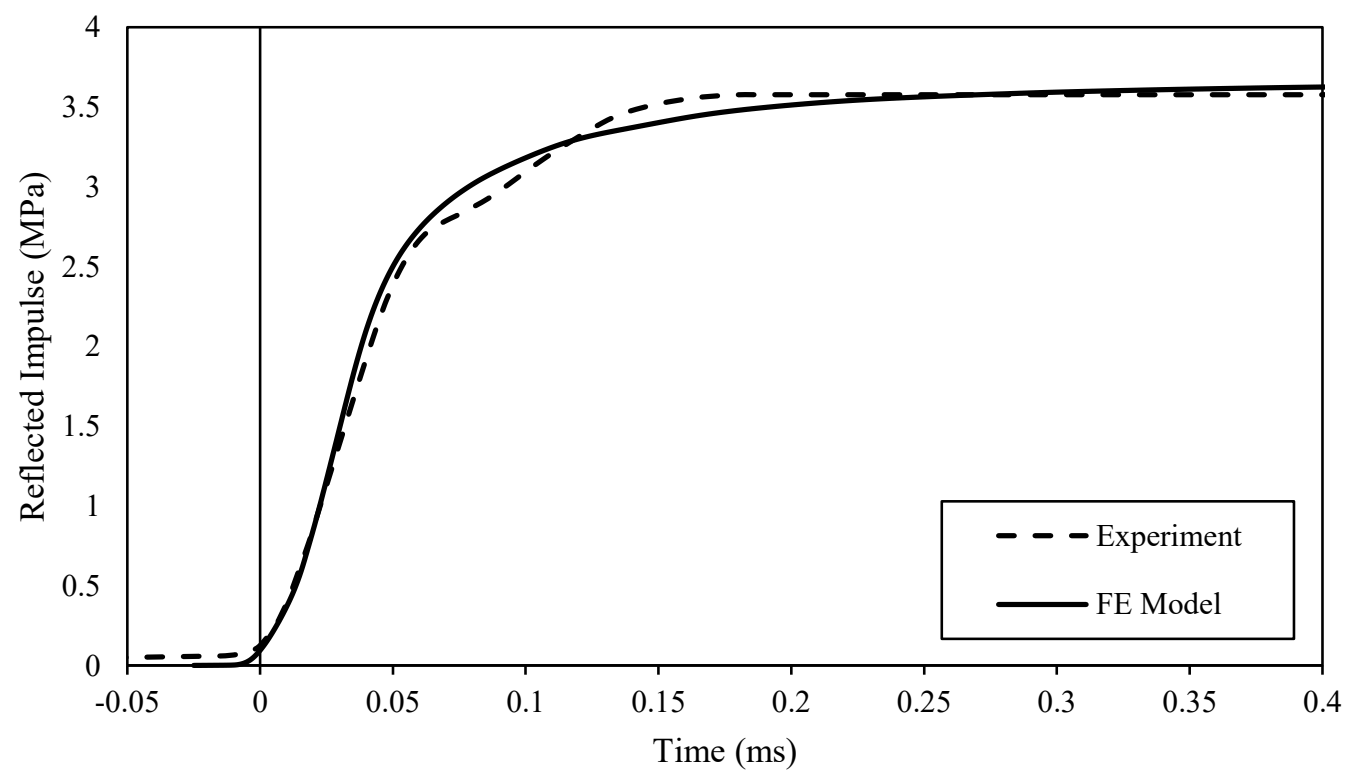

Figure 50: Comparison of analytical and experimental impulse-time histories for 5.6 kg ANFO with a $500 \mathrm{~mm}$ stand-off distance $\left(\mathrm{z}=0.282 \mathrm{~m} / \mathrm{kg}^{1 / 3}\right)$. 


\subsection{Validation of Structural Response under Near-Field Blast Loading}

Given that the model has been proven to be able to capture blast pressure and impulse generated in the near-field, a verification on its ability to model structural response to near-field loads is conducted.

\subsubsection{Validation against Yang et al. (2019) Test}

\subsubsection{Experiment Details}

The earlier discussed experimental study by Yang et al. (2019) is also used to verify the ability of the FE model to compute the structural response under near-field blast loads. The response of slab $\mathrm{NC}-3$ was used for this verification. As previously mentioned, this slab was subjected to $5.6 \mathrm{~kg}$ of ANFO at a distance of $500 \mathrm{~mm}$ resulting in a scaled distance of $\mathrm{z}=0.282 \mathrm{~m} / \mathrm{kg}^{1 / 3}$. Experimental setup and structural details of the slab are provided in Section 5.1.2.1.

\subsubsection{FE Model}

The 2D and 3D FE models used for this verification are shown earlier in Figure 47 and Figure 48. The 2D model was terminated prior to impingement onto the structure, at which point the data was mapped into the 3D model containing the RC slab.

The slab was modelled using CSCM concrete (MAT_CSCM_CONCRETE) assigned to solid elements with a mesh size of $10 \mathrm{~mm}$, resulting in a total of 100,000 . For erosion of concrete, the ERODE parameter was set to 1.3 for this model. The steel reinforcing bars were modelled using an elastoplastic material type (MAT_PIECEWISE_LINEAR_PLASTICITY) assigned to beam elements with a mesh size of $10 \mathrm{~mm}$. To provide a more realistic representation of the support condition, the end steel plate on the bottom of the slab was modelled using solid elements with a mesh size of $10 \mathrm{~mm}$. The material type was similar to that used for the steel reinforcing bars. The steel support was constrained from translation or rotation in all directions (ie. fully fixed), while 
the slab was free to move and interact with the steel plate. The interaction of the slab and plate was defined using CONTACT_AUTOMATIC_SURFACE_TO_SURFACE, as described in Section 4.1.3.

\subsubsection{Results and Discussion}

A mesh sensitivity analysis was conducted for the modelling of the air in 3D. The results of this analysis are presented in Figure 51. The results for the displacement of the slab converge at an air mesh size of $20 \mathrm{~mm}$. The run times for the analyses for various mesh sizes are reported in Table 9. Further reducing the mesh size has little effect on the predicted response of the slab but has a significant increase in analysis run time and total number of elements. A graphical representation of this data is also presented in Figure 52.

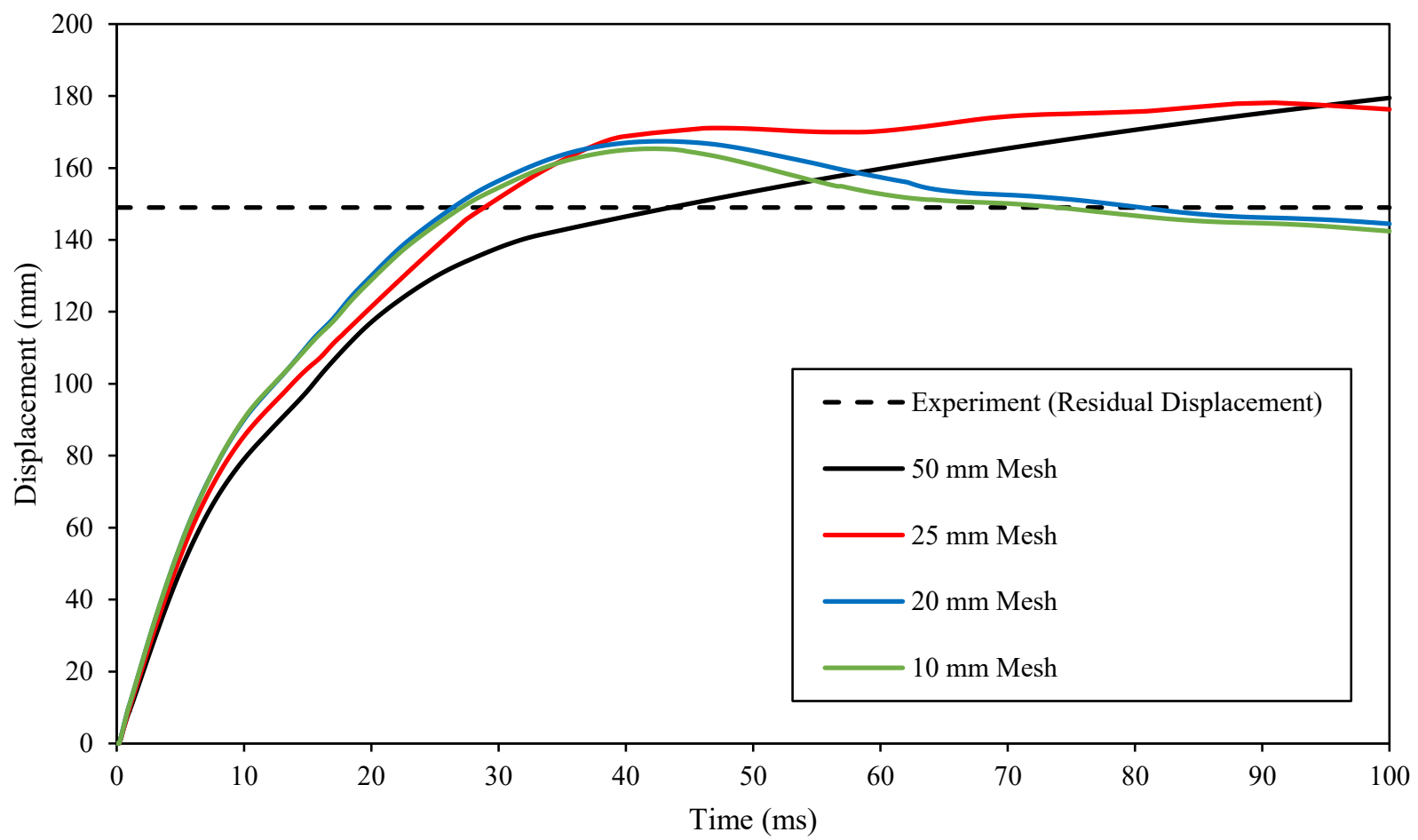

Figure 51: Mesh sensitivity analysis for modelling the air in 3D. 
Table 9: Analysis times for various mesh sizes of air in 3D.

\begin{tabular}{c|c|c} 
Mesh Size (mm) & No. of Elements & Analysis Time (min) \\
\hline 50 & 41600 & 146 \\
25 & 332800 & 161 \\
20 & 650000 & 167 \\
10 & 5240000 & 422
\end{tabular}

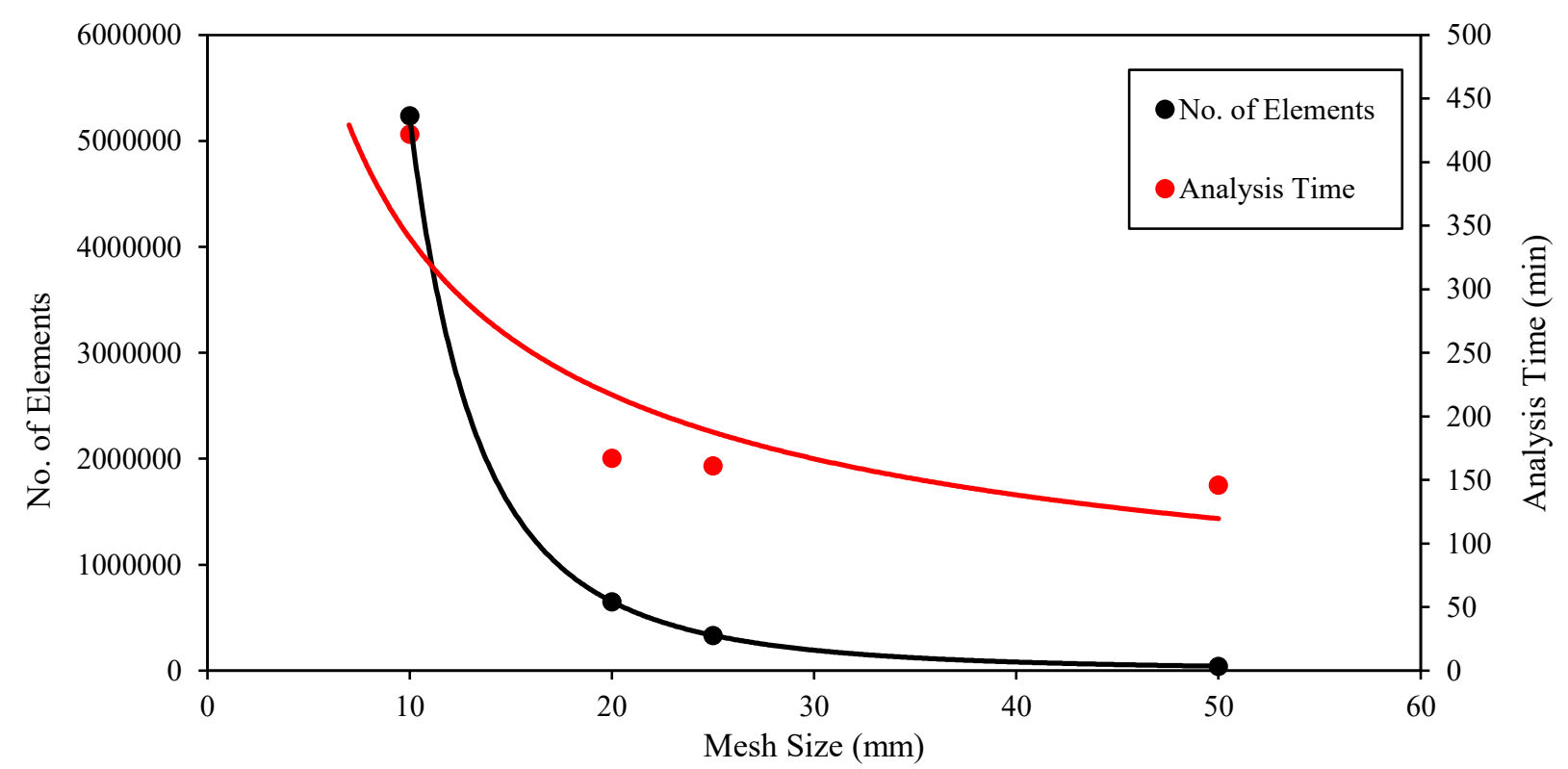

Figure 52: Analysis time and total number of elements for various 3D air mesh sizes.

Figure 53 compares the displacement-time history of the centre of the slab computed by the FE model to the permanent deflection of the slab measured in the experiment, which was reported to be $149 \mathrm{~mm}$. The analytical displacement-time history was predicted using a $20 \mathrm{~mm}$ 3D air mesh size. The FE model shows good agreement with the experimental result predicting a permanent deflection of $145 \mathrm{~mm}$. The experimental study did not report the displacement-time history so only the permanent deflection was compared. 


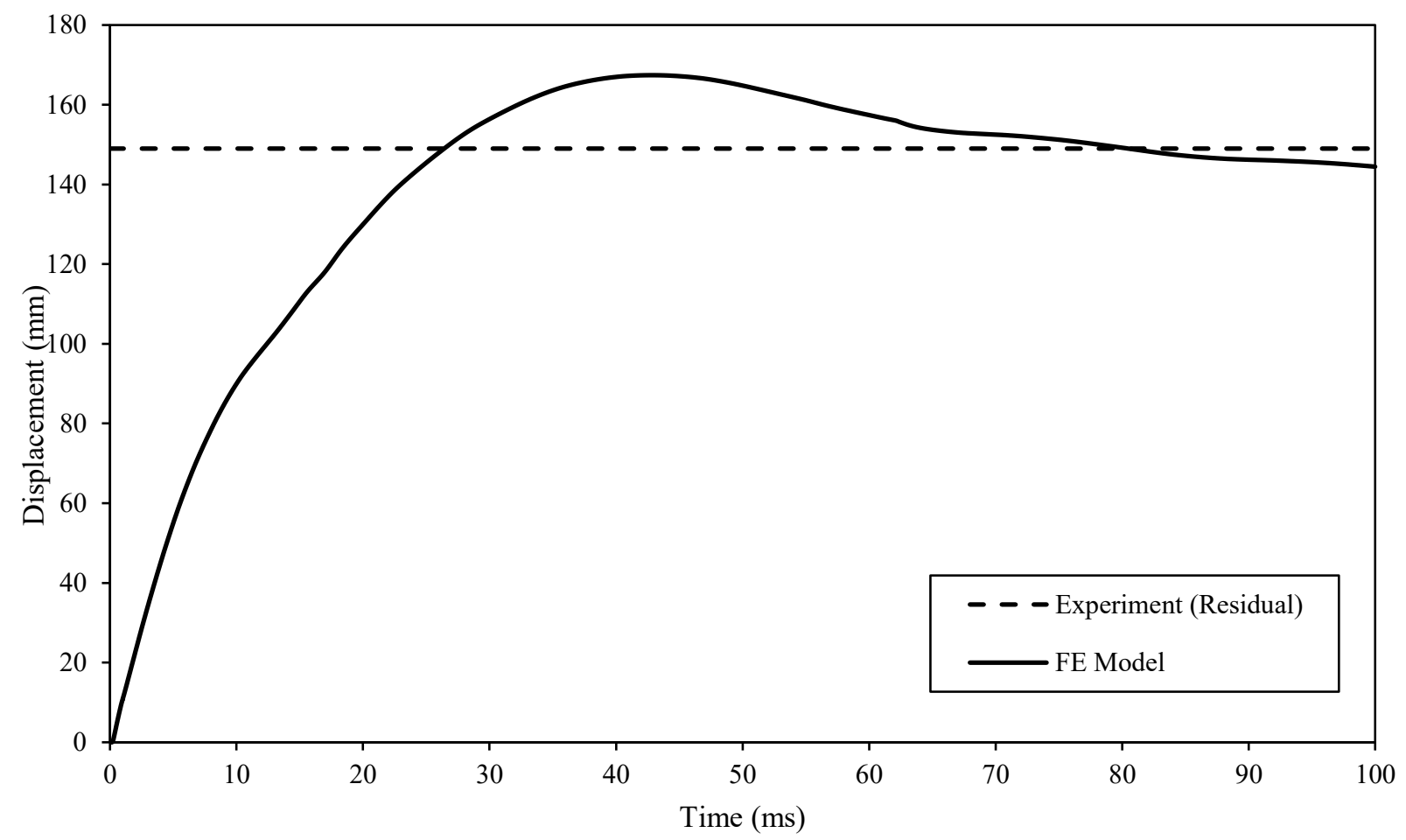

Figure 53: Displacement-time history results for slab NC-3.

Figure 54 compares the experimental and analytical deflected shapes and crack patterns. The plastic strain distribution in Figure 54c is based on the scalar damage parameter, $d$, defined in CSCM. The amount of plastic strain developed in an element is used to measure its level of damage. Red areas $(d=0.99)$ indicate fully damaged elements, while blue areas $(d=0)$ indicate undamaged elements. Elements that have a scaled damage value of $d=0.99$ have lost all their strength and stiffness.

In the experiment, the slab failed due to severe tensile damage of concrete in the distal face with cracks extending to the incident face of the specimen and extensive yielding in the tension reinforcement which indicates a flexural failure mode. It can be seen from Figure 54 that the FE model was able to capture the deflected shape and crack pattern accurately. In the FE model, the concrete along the midspan of the slab showed significant damage due to high tensile strains that extended through the thickness, representing a large crack, similar to that observed in the 
experiment. Figure 55 plots the effective strain distribution in the slab once it had come to rest after the blast. The effective strain indicates absolute strain values without any differentiation between tensile and compressive strains. Figure 55a shows a mostly undamaged incident face with a cross-shaped crack developed at the center and extended to the edges at the mid-span. Figure 55b shows the distal face which had a high degree of tensile damage compared to the incident face. The distal face shows both radial cracks extending from the centre as well as some concentric cracks. The distal face of the blast-damaged slab was not reported in the experiment. However, the FE model shows large radial cracks extending from the centre of the slab to each corner at approximately $45^{\circ}$. Large cracks are also visible near the midspan of the slab running parallel to the supports. The crack located exactly at the midspan extends from one end of the slab to the other. Figure 56 plots the effective stress distribution in the slab at peak response. Similar to the effective strain, the effective stress does not differentiate between tensile and compressive stresses. Under the loading conditions, the DIF for the concrete in compression was computed as approximately 1.57 , resulting in a dynamic concrete compressive strength of $55 \mathrm{MPa}$. The compressive stresses in the concrete on the incident face are significantly less than the dynamic strength, indicating a mostly undamaged incident face. This is consistent with the observations concluded regarding the effective strain distribution. 


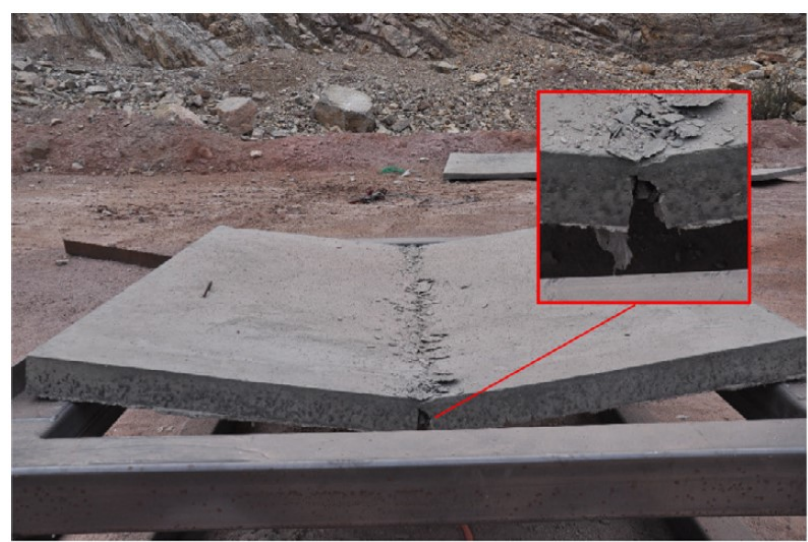

(a)

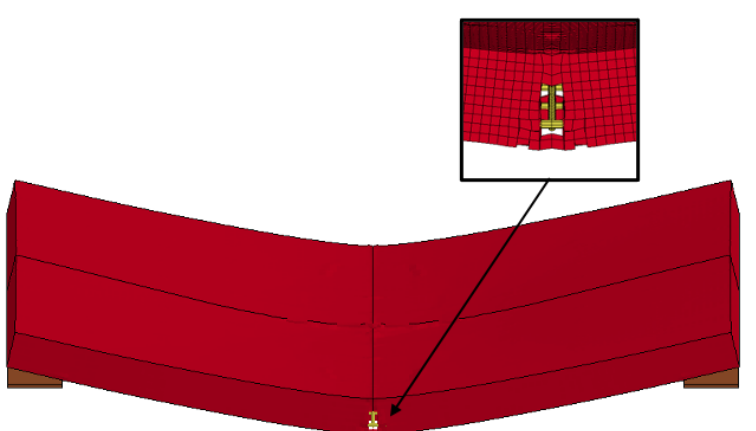

(b)

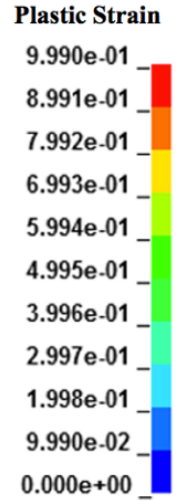

(c)

Figure 54: Comparison of the deflected shapes and damage patterns for slab NC-3: (a) experimental results (Yang et al., 2019), (b) deflected shape predicted by the FE model, and (c) FE model deflected shape with plastic strain (damage) distribution.

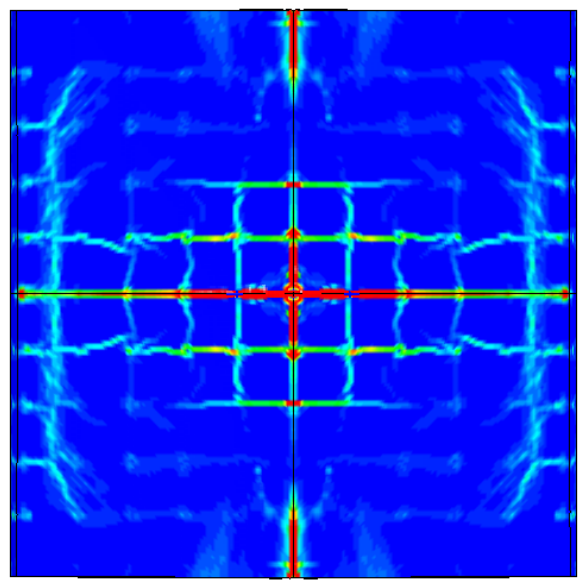

(a)

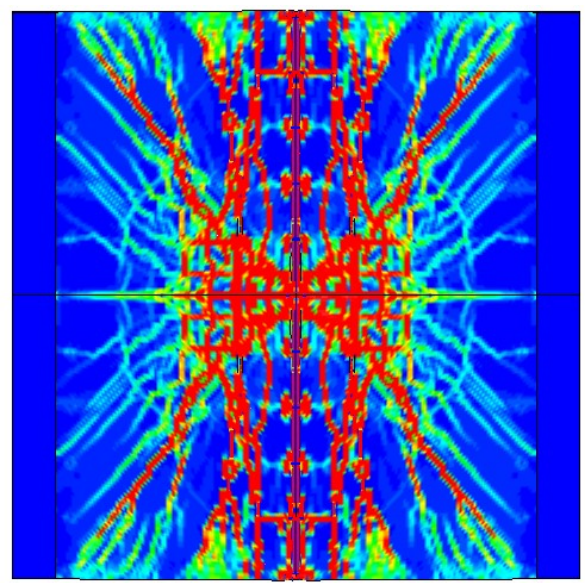

(b)

\section{Effective Strain}

$3.500 \mathrm{e}-02$

3.150 e-02

2.800e-02

$2.450 \mathrm{e}-02$

2.100e-02

1.750 e-02

1.400 e-02

$1.050 \mathrm{e}-02$

$7.000 \mathrm{e}-03$

$3.500 \mathrm{e}-03$

$0.000 \mathrm{e}+00$

Figure 55: Effective strain distribution in the blast-damaged slab on the (a) incident face and (b) distal face. 


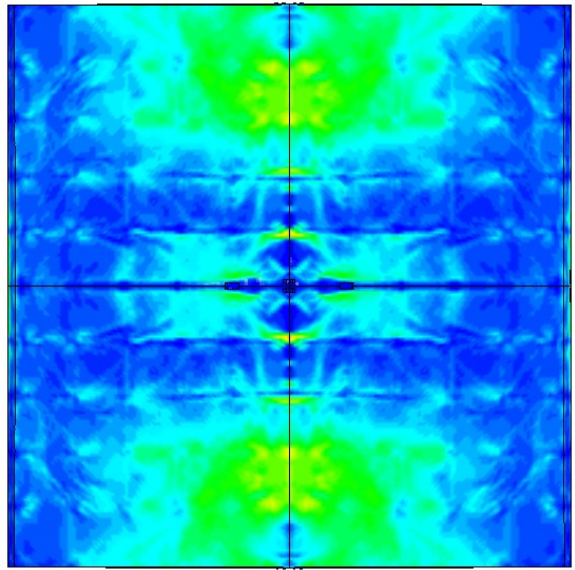

(a)

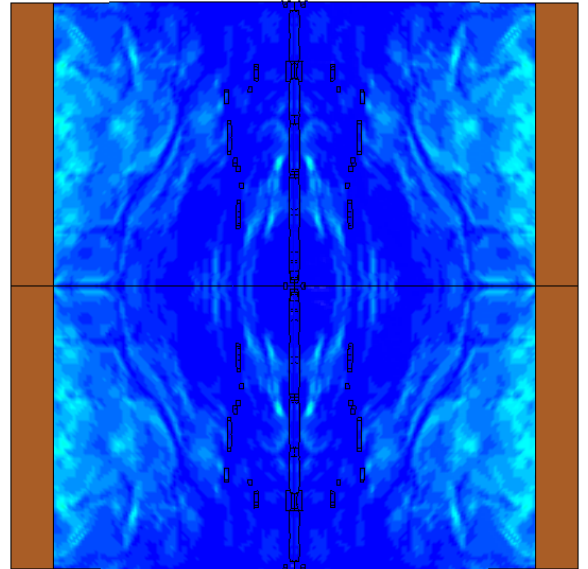

(b)
Effective Stress

(Gpa)

$5.480 \mathrm{e}-02$

$4.932 \mathrm{e}-02$

4.384e- 02

$3.836 \mathrm{e}-02$

$3.288 \mathrm{e}-02$

$2.740 \mathrm{e}-02$

2.192e-02

$1.644 \mathrm{e}-02$

$1.096 \mathrm{e}-02$

$5.480 \mathrm{e}-03$

$0.000 \mathrm{e}+00$

Figure 56: Effective stress distribution in the slab at peak response on the (a) incident face and (b) distal face.

The axial strain distribution in the steel reinforcement is presented in Figure 57. Only the yield strength and Young's modulus of the steel were reported in the study. Therefore, a bilinear stressstrain response was used for the steel reinforcement. Under the loading conditions, the DIF for the steel reinforcement due to high strain rate was computed to be approximately 1.29 , which results in a dynamic yield strength and strain of $303 \mathrm{MPa}$ and 0.0015 , respectively. Figure 57a shows the distribution of yielded steel elements based on the dynamic yield strain. While the study did not report the condition of the reinforcing steel, the FE model shows yielding for a large portion of the steel mesh. In Figure 57b, the elements along the midspan of the slab show axial strain values approaching approximately 150 millistrain, indicating probable rupture of steel reinforcement. The line of ruptured elements extends along the midspan from one edge of the slab to the other. The ruptured steel aligns with the large crack along the midspan of the concrete shown in the experimental results of Figure 54a. 


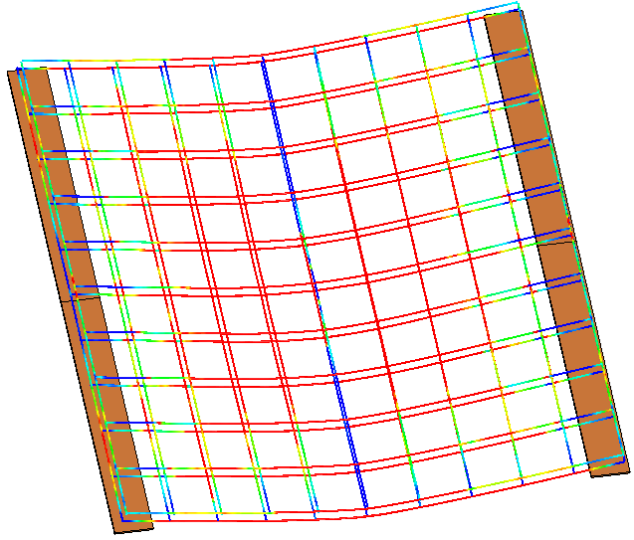

(a)

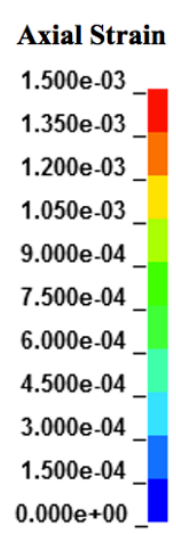

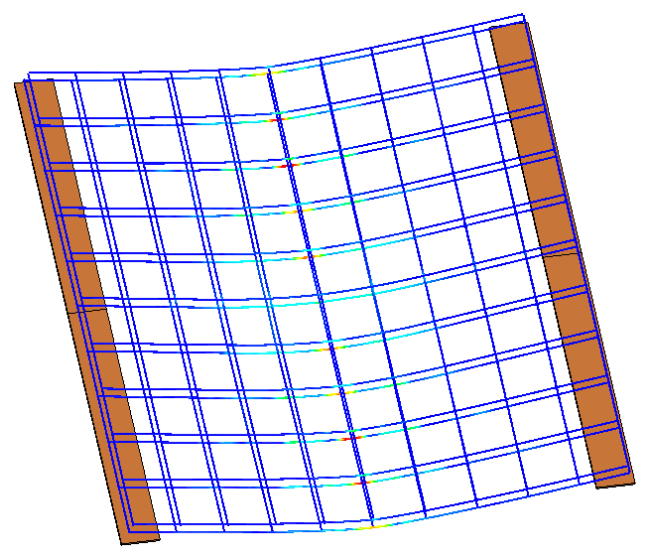

(b)

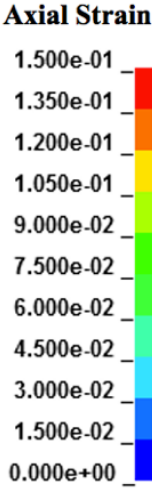

Figure 57: Axial strain distribution in steel reinforcement post-blast showing (a) yielded elements and (b) ruptured elements.

\subsubsection{Validation against Wang et al. (2012) Test}

\subsubsection{Experiment Details}

A second validation was completed using an experimental study conducted by Wang et al. (2012). The study assessed the explosion resistance of square RC slabs under close-in blast loads. The authors tested several slabs at different scaled distances to examine different damage levels. The peak displacement at the centre of the slab and the spall radius on the distal face of the damaged slab were measured in the test.

From the experiment, Slab E was selected to validate the FE model. This slab was selected as the test specimen was a full-scale RC slab, whereas the other tests were scaled specimens. Furthermore, this slab was subjected to a higher charge mass and suffered a greater degree of damage, which would be useful when comparing the results of the FE model to those reported in the experiment. The experimental setup is shown in Figure 58. For this test, a cylindrical charge of $0.64 \mathrm{~kg}$ of TNT was suspended above the centre of the slab at a distance of $500 \mathrm{~mm}$, giving a scaled distance of $\mathrm{z}=0.591 \mathrm{~m} / \mathrm{kg}^{1 / 3}$. The cylinder had a diameter-to-height ratio of approximately 2 and it was detonated from the top. The stand-off distance was measured from the centre of the explosive to the top surface of the slab. 


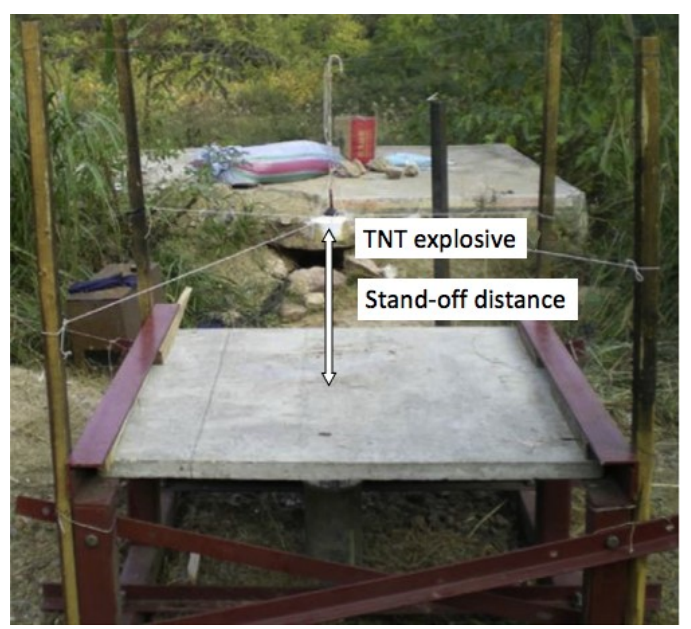

Figure 58: Field test setup of the RC slab under near-field explosion (Wang et al., 2012).

The slab dimensions and reinforcement detailing are shown in Figure 59. The concrete had an average uniaxial compressive strength of $39.5 \mathrm{MPa}$ and the reinforcing steel had a yield strength of $600 \mathrm{MPa}$. The reinforcement consisted of $6 \mathrm{~mm}$ diameter steel and was spaced at a distance of $75 \mathrm{~mm}$. The member was intended to be fully fixed by the support system which consisted of 8 mm thick steel angles which were clamped down. As seen in Figure 58, the steel angles were slightly too large for the thickness of the slab. Therefore, pieces of wood were inserted between the steel angles and the slab. As a result, the actual fixity of the support was somewhere between fixed and pinned. Therefore, it should be noted that even a small deformation in the steel angles or movement in the wood can affect the level of fixity at the supports.

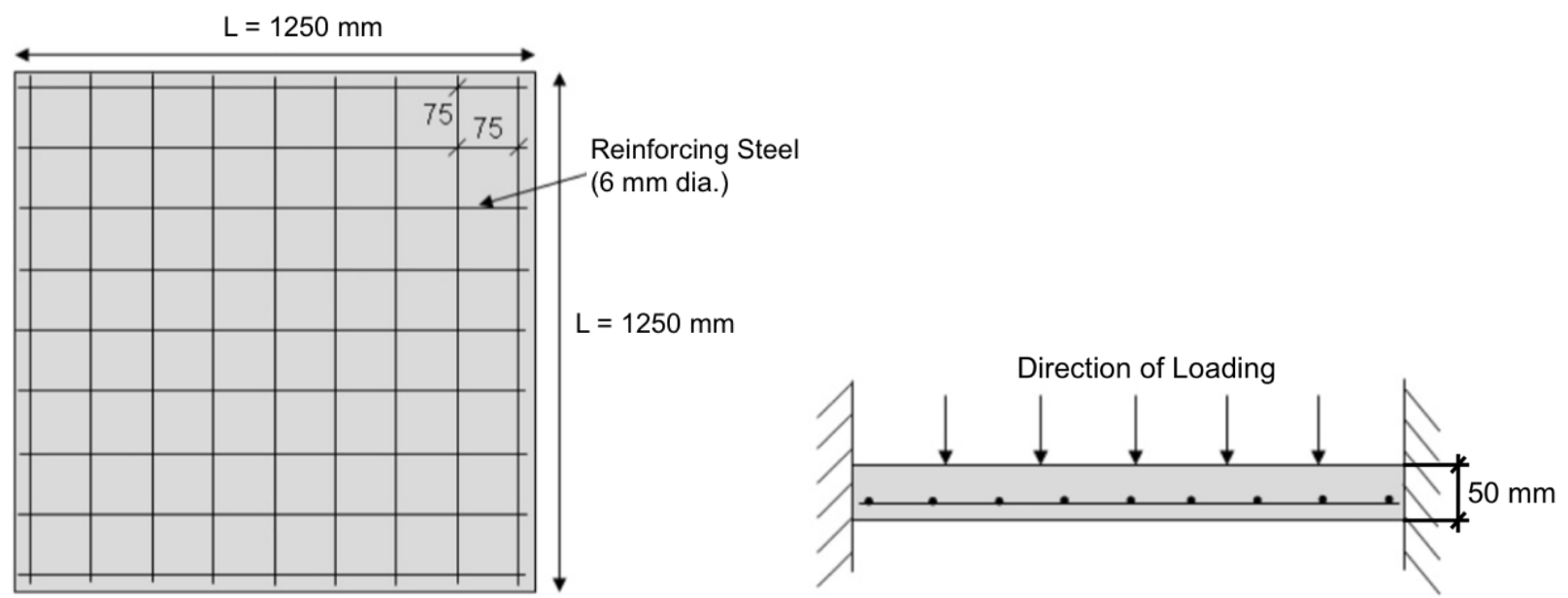

Figure 59: Geometry and reinforcement details of the RC slab (Wang et al., 2012). 


\subsubsection{FE Model}

For this verification study, a 3D-to-3D mapping approach was used in order to model the cylindrical shape of the explosive. The explosive was detonated first in a quarter-symmetric 3D model containing only the air and the explosive, as shown in Figure 60a. The air in this model had a mesh size of $2 \mathrm{~mm}$ to ensure accurate modelling of the detonation process, giving a total of $6,407,469$ solid elements. The analysis was terminated prior to impingement on the structure and the data was mapped into a second 3D model containing the RC slab, as shown in Figure 61a. This 3D model was also quarter-symmetric and had an air mesh size of $20 \mathrm{~mm}$, giving a total of 222,264 solid elements. The blast wave was allowed to interact with the RC slab, which was modelled using solid elements with a mesh size of $5 \mathrm{~mm}$, for a total of 156,250 solid elements, and the CSCM concrete material model. While the mesh sensitivity analysis for concrete showed a mesh size of $10 \mathrm{~mm}$ to be sufficient, a smaller mesh size was selected to capture the local damage of concrete more accurately. For erosion of concrete, the ERODE parameter was set to 1.2 for this model. The steel rebar and the steel plates were modelled using the elastoplastic material model described in Section 4.1.2 with a mesh size of $10 \mathrm{~mm}$. The interaction of the slab and plates was modelled using CONTACT_AUTOMATIC_SURFACE_TO_SURFACE, as described in Section 4.1.3. The steel plates were fully fixed from translation and rotation in all directions while the slab was free to move and interact with them. 


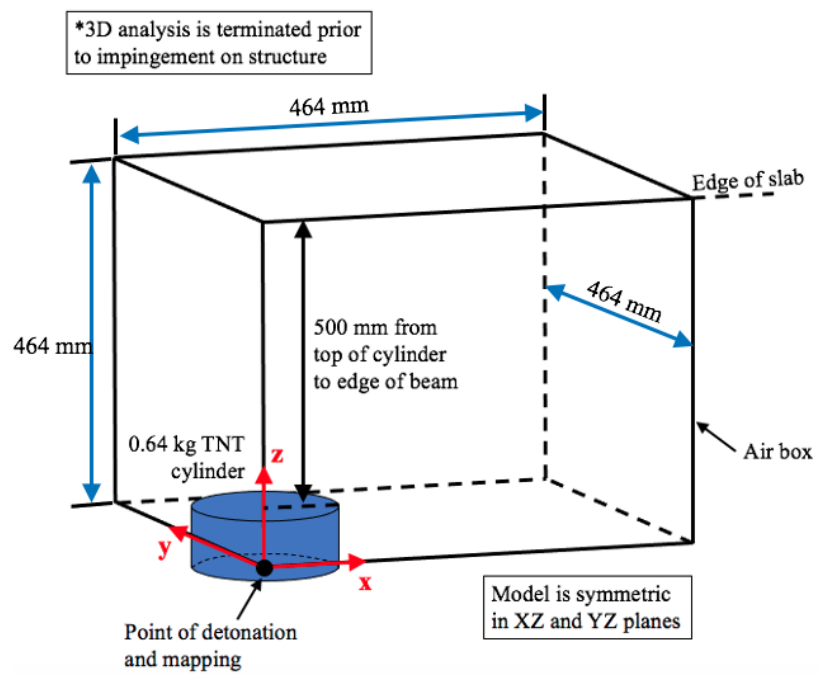

(a)

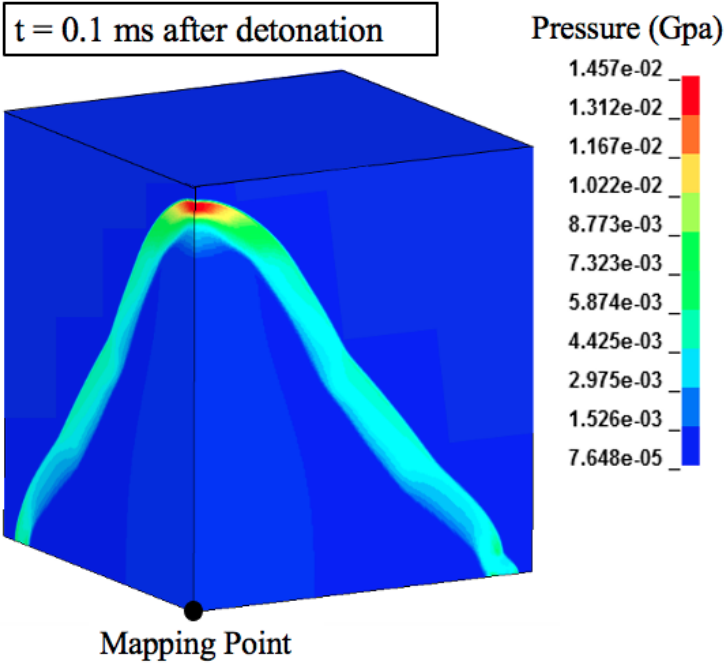

(b)

Figure 60: (a) 3D model where TNT cylinder is detonated (b) Pressure distribution just prior to mapping.

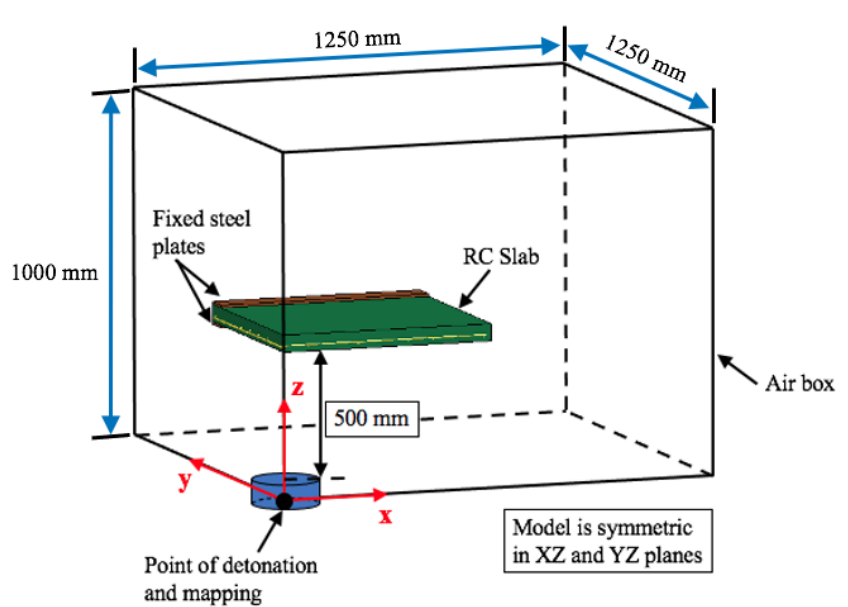

(a)

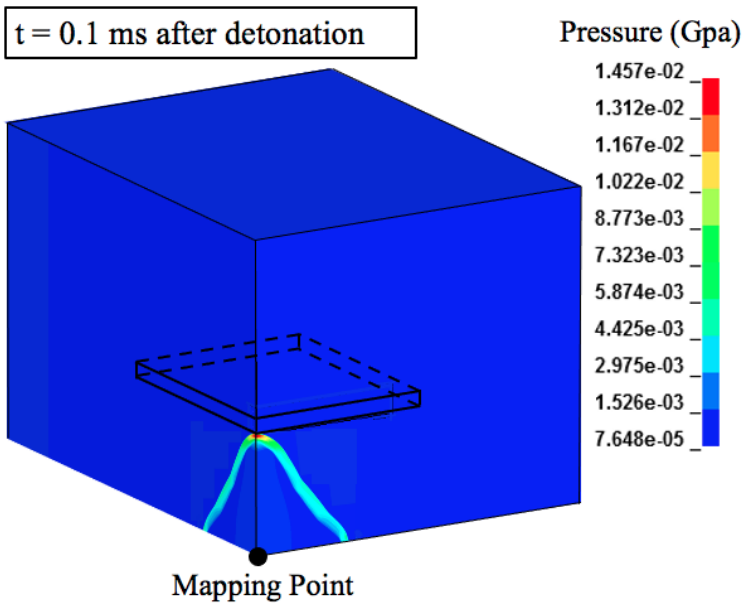

(b)

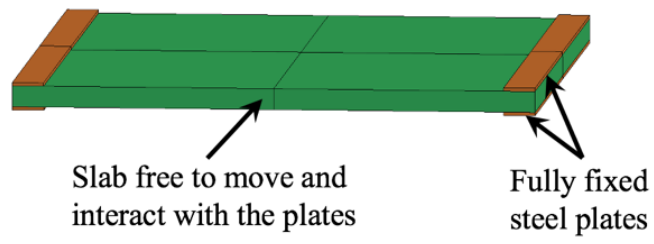

(c)

Figure 61: (a) 3D model where blast wave interacts with the slab (b) Mapping of pressures just prior to interaction (c) Support condition of the slab (fixed support). 


\subsubsection{Results and Discussion}

Figure 62 shows the incident and the distal face of the damaged slab specimen reported in the experiment. In comparison, Figure 63 shows the damage profile of the slab as predicted by the FE model. Based on these results, the damage profile predicted by the FE model corresponds very well with the experimentally recorded damage.

The slab profile computed by the FE model in Figure 63a shows a mostly undamaged incident face. A very small area of moderate damage is observed at the centre of the slab, which coincides with the notch seen in the centre of the slab in Figure 62a. The FE model also shows some damaged elements in a circular pattern, which coincides with the concentric cracks observed on the incident face of the experimental results. However, the FE model also shows some damaged elements at the supports. Potential damage to the areas under the supports is not clearly visible in the figures taken from the test specimen, so this could not be corroborated with experimental data.

Figure $63 \mathrm{~b}$ shows the damage on the distal face of the slab as predicted by the FE model. A large degree of localized damage due to spalling is visible at the centre of the slab. This is in line with the spalling reported from the experiment as shown in Figure 62b. The FE model also captures the radial cracks seen extending from the centre of the slab to the edges in Figure 62b.

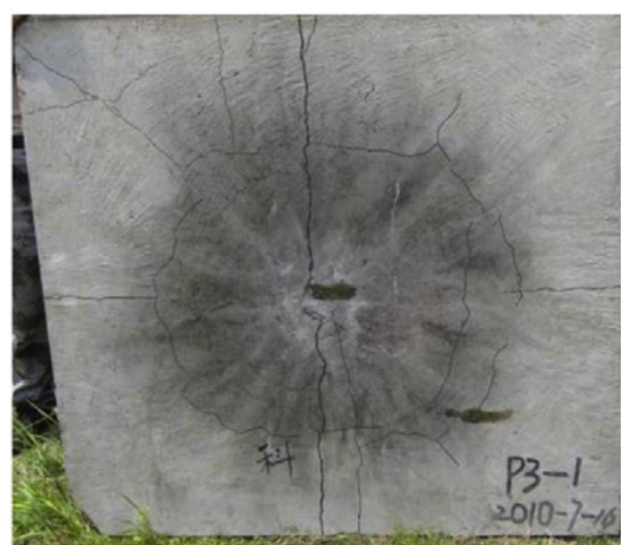

(a)

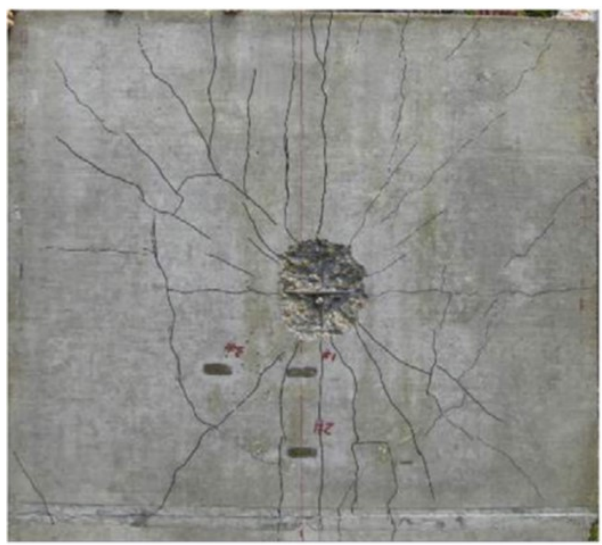

(b)

Figure 62: Experimental damage to Slab E on the (a) incident face and (b) distal face (Wang et al., 2012) 


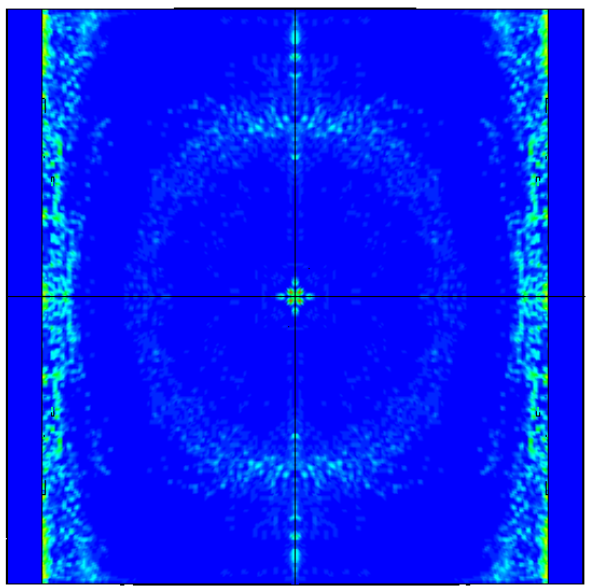

(a)

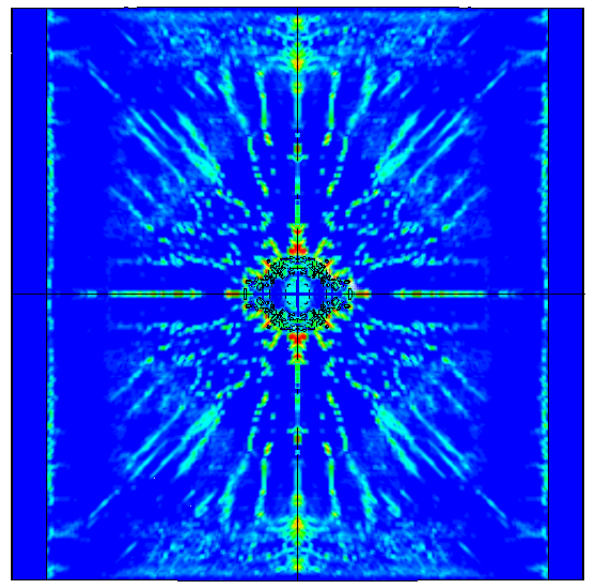

(b)
Plastic Strain

$9.990 \mathrm{e}-01$

8.991e-01

7.992e-01

$6.993 \mathrm{e}-01$

$5.994 \mathrm{e}-01$

4.995e-01

$3.996 \mathrm{e}-01$

2.997e-01

$1.998 \mathrm{e}-01$

$9.990 \mathrm{e}-02$

$0.000 \mathrm{e}+00$

Figure 63: Plastic strain (damage) distribution predicted by the FE model of Slab E on the (a) incident face and (b) distal face.

The effective stress distribution in the slab at peak response is presented in Figure 64. Under the loading conditions, the DIF for the concrete was computed to be approximately 1.97 , resulting in a dynamic compressive strength of $78 \mathrm{MPa}$. It can be seen from Figure 64a that the incident face of the slab is under a high degree of stress with compressive stresses in concrete reaching values greater than $50 \mathrm{MPa}$ along the midspan of the slab. While this is higher than the uniaxial compressive strength of the concrete $\left(\mathrm{f}_{\mathrm{c}}{ }^{\prime}=39.5 \mathrm{MPa}\right)$, the concrete elements in this region did not experience crushing because of the strength increase due to high strain effects. The effective strain distribution in Figure 65a also shows a mostly undamaged incident face. However, there is a line of high strain extending along the midspan of the slab, which shows strain values significantly higher than the cracking strain of concrete. This high strain region coincides with the high stress at the midspan of the slab as well as with the crack visible in the experiment (see Figure 62a).

As for the distal face of the slab, the blue stress contours in Figure $64 \mathrm{~b}$ shows concrete elements subjected to tensile stress. This distribution coincides with the effective strain presented in Figure 65a. The distal face shows a localized area of concrete that is subjected to tensile stress and high strain, corresponding with the spalling seen in the experimental results. The effective strain also 
shows radial lines extending from the centre of the slab to the edges representing the radial cracks reported in the experiment.

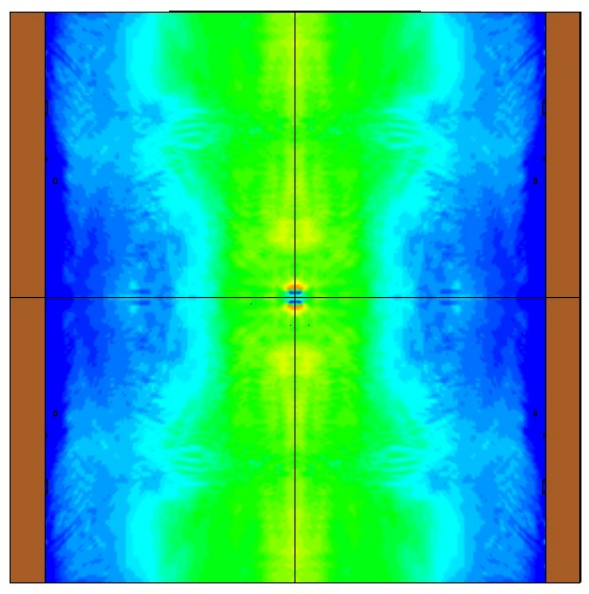

(a)

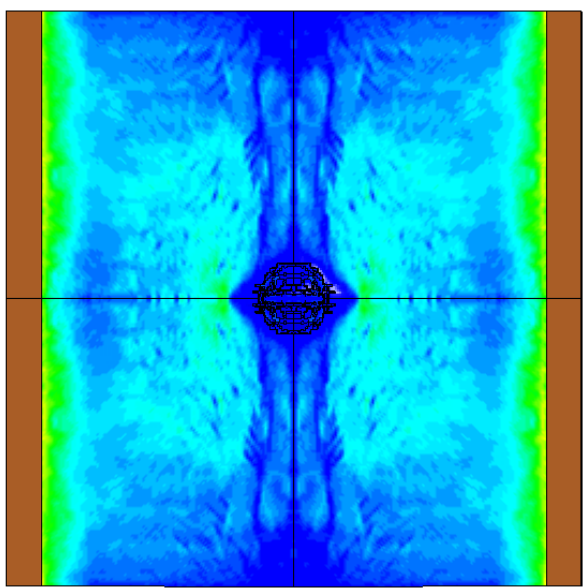

(b)

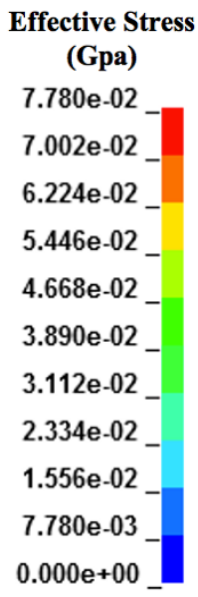

Figure 64: Effective stress distribution in the slab at peak response on the (a) incident face and (b) distal face.

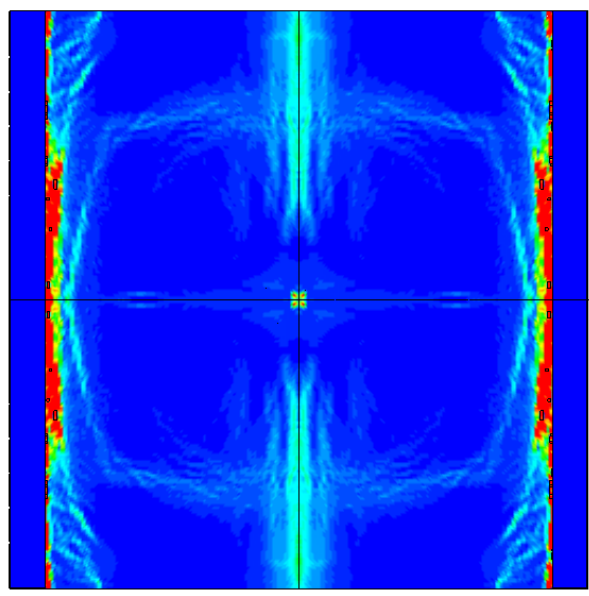

(a)

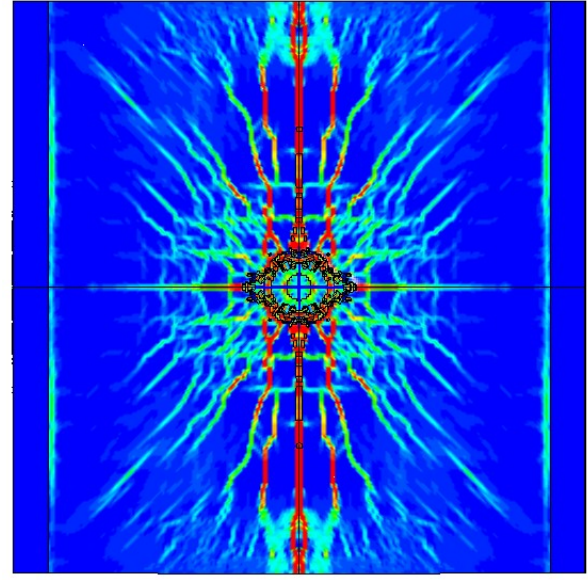

(b)

Effective Strain
$3.500 \mathrm{e}-02$
$3.150 \mathrm{e}-02$
$2.800 \mathrm{e}-02$
$2.450 \mathrm{e}-02$
$2.100 \mathrm{e}-02$
$1.750 \mathrm{e}-02$
$1.400 \mathrm{e}-02$
$1.050 \mathrm{e}-02$
$7.000 \mathrm{e}-03$
$3.500 \mathrm{e}-03$
$0.000 \mathrm{e}+00$

Figure 65: Effective strain distribution in the blast-damaged slab on the (a) incident face and (b) distal face.

Figure 66 shows the axial strain distribution in the steel reinforcement. Only the yield strength of the reinforcing steel was provided in the study, so a bilinear stress-strain response was used with Young's modulus assumed to be $200 \mathrm{GPa}$. Under the loading conditions, the DIF of the steel reinforcement was computed to be approximately 1.16, resulting in a dynamic yield strength and strain of $696 \mathrm{MPa}$ and 0.00348 , respectively. The condition of the steel reinforcement was not 
reported in the study, but based on the computed dynamic yield strain, the FE model indicates that a large portion of the steel reaches yield strength (see Figure 66a). The maximum strains computed in the steel reinforcement are shown in Figure 66b. The low magnitude of the strains indicate that no rupturing of steel reinforcement occurred.

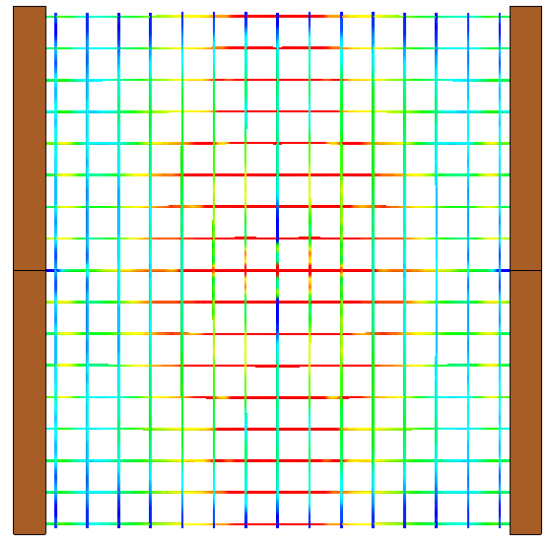

(a)

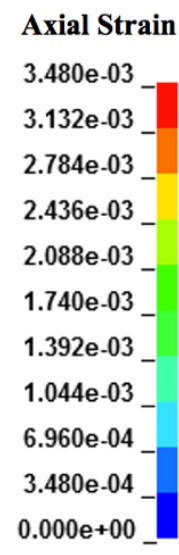

Figure 66: Axial strain distribution in the steel reinforcement showing (a) dynamic yield strain and (b) maximum strains.

In the experiment, the authors measured the spalled area to have a diameter of $120 \mathrm{~mm}$. Figure 67 shows a close-up of the damage on the distal face of the slab computed by the FE model. The radius shown measures the distance from the centre of the slab to the last fully damaged element (where $d=0.99$ ). This radius was measured to be $120 \mathrm{~mm}$ and represents the spall damage of the distal face. The few fully damaged elements that extend past the outlined radius likely represent the transition into the cracks that emanate from the centre of the spalled area. It can be concluded that the damage formulation in CSCM was able to accurately capture the extent of the concrete spalling on the distal face of the slab. 

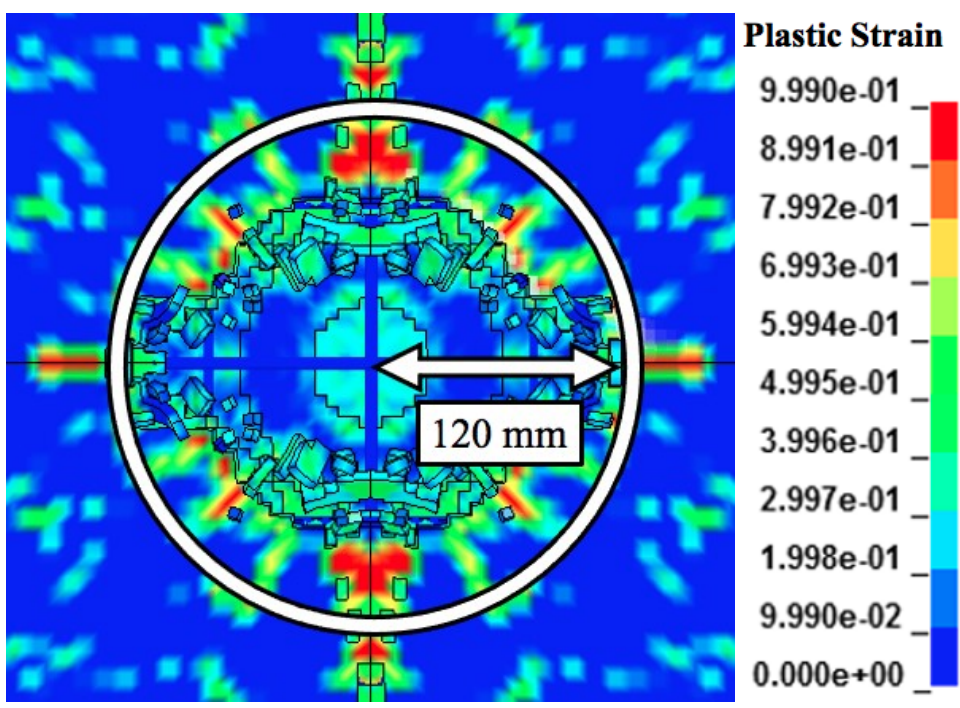

Figure 67: Plastic strain distribution on distal face of the slab with approximate spalling radius.

As previously mentioned, the authors in this experiment also collected central deflections of the slabs. However, no information is provided regarding the test instrumentation and how the deflections were collected. By investigating other similar experimental studies conducted by the authors (Zhang et al., 2013), it was ascertained that the study likely used a cluster of steel needles to determine the maximum deflection of the slab. This device is somewhat visible in Figure 58 beneath the slab. However, the placement of these needles in reference to the centre of the slab is unknown. Based on the setup shown in Figure 58, it seems that the needles may have been placed directly beneath the centre of the slab. If this is the case, the severe spalling on the distal face of the slab could potentially have resulted in loss of contact with the needles, underestimating the peak central deflection. Ultimately, the details regarding slab displacement collection are not provided, and therefore the displacement results were not compared. Furthermore, very little discussion was provided on the actual support system of the slab. The authors mentioned that the fixity of the slab was somewhere between fixed and pinned. As a result of these uncertainties, there was not a high degree of confidence in the displacement results. Therefore, the results of this 
validation were not intended to verify global structural response, rather focused on local response which is more critical in near-field blast loadings.

The exact same slab specimen from this experimental study was analyzed with two additional FE models to demonstrate the capability of CFD modelling method for performance assessment under near-field blast loadings and also emphasize the importance of accurate CFD modelling. Figure 68 compares the results of the same experiment when modelled using LBE, CFD with a spherical charge, and CFD with a cylindrical charge. All other modelling parameters were kept constant, such as charge mass and stand-off distance, material parameters, mesh sizes, support conditions, etc. Only the modelling method for the blast loading was varied. 

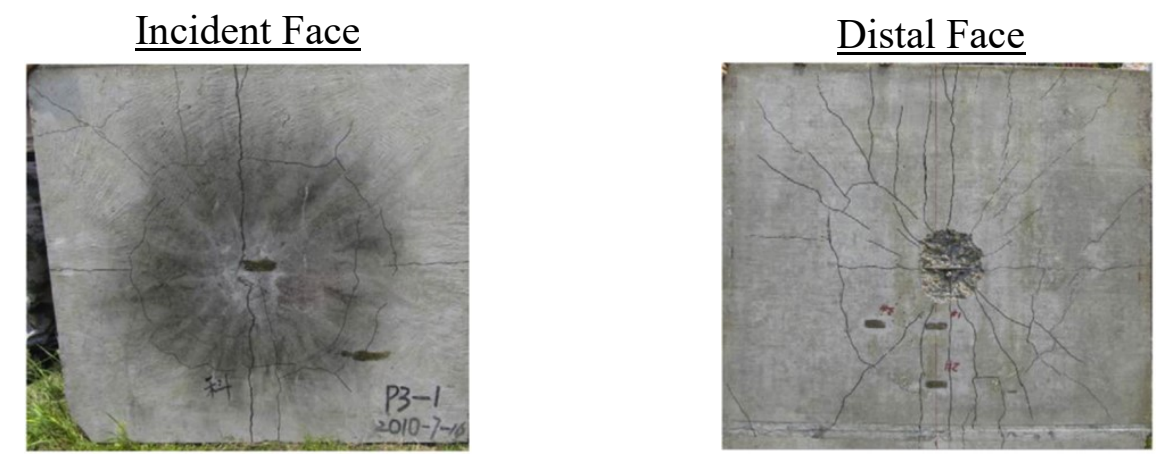

(a) Experiment

\section{Distal Face}
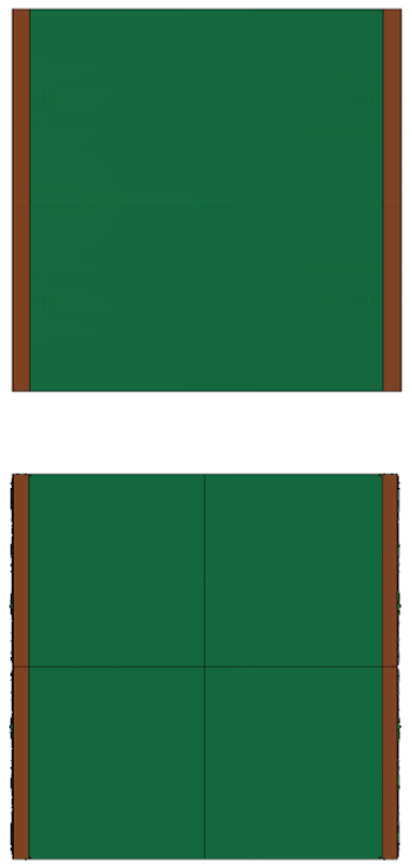

(c) CFD modelling method with spherical charge
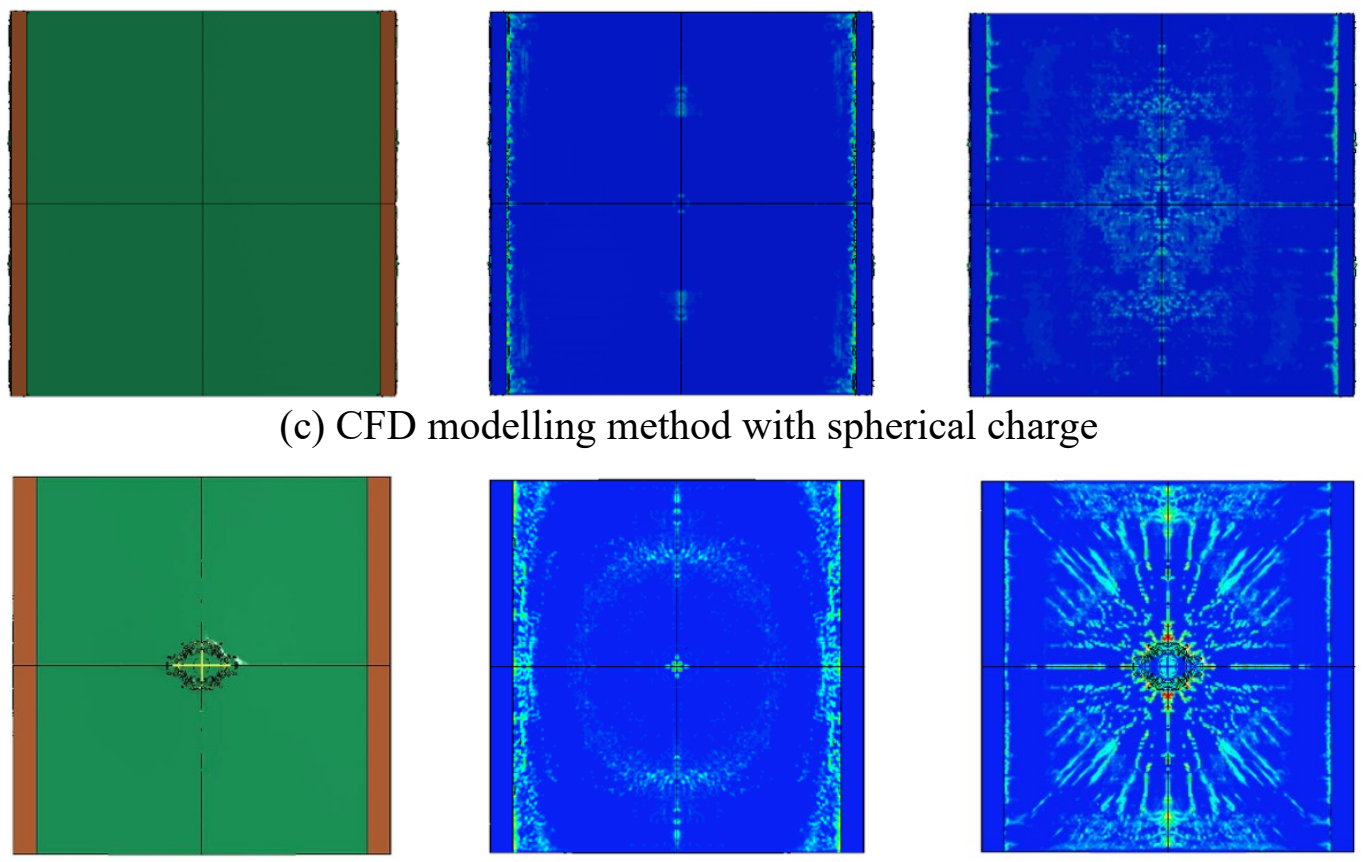

Plastic Strain

$9.990 \mathrm{e}-01$

8.991e-01_

7.992e-01_

$6.993 \mathrm{e}-01$

$5.994 \mathrm{e}-01$

$4.995 \mathrm{e}-01$

$3.996 \mathrm{e}-01$

2.997e-01

$1.998 \mathrm{e}-01$

$9.990 \mathrm{e}-02$

$0.000 \mathrm{e}+00$

(d) CFD modelling method with cylindrical charge

Figure 68: Slab damage reported (a) in the experiment, (b) using LBE modelling method, (c) using CFD modelling method with spherical charge, and (d) using CFD modelling method with cylindrical charge. 
The LBE function uses the CONWEP program which is an implementation of the empirical blast models of Kingery and Bulmash to predict the loading on the structure (Randers-Pehrson \& Bannister, 1997). As previously discussed, the empirical models available to predict blast loadings have been reported to be inaccurate for scaled distances less than $0.4 \mathrm{~m} / \mathrm{kg}^{1 / 3}$. Figure $68 \mathrm{~b}$ shows the damage profile of the slab post-blast when using LBE. The incident and distal faces of the slab are also presented with the plastic strain distribution. On the incident face, the slab shows some damage under and near the supports but nowhere else. As for the distal face of the slab, the results show no clear spalling. With the plastic strain distribution, there is a low-to-moderate degree of damage all over the slab. The analysis failed to capture local damage or spalling concentrated at the centre of the test specimen or compute the radial cracks reported in the experiment. While the scaled distance for this test was greater than $0.4 \mathrm{~m} / \mathrm{kg}^{1 / 3}$, the explosive used was a cylindrical charge mass. As previously discussed, charge shape can have a significant influence on the magnitude and spatial distribution of pressure in the near-field. Since LBE does not have the option to model different explosive shapes, it was not able to accurately capture the response of the slab. Figure 68c shows the results of the same experiment when modelled using CFD, but with incorrect explosive shape. The charge shape in the experiment was cylindrical, but the results presented in Figure $68 \mathrm{c}$ were attained using a spherical charge shape. In Figure $68 \mathrm{c}$ with the plastic strain distribution, some damage is visible under the supports on the incident face of the slab. However, no damage is seen anywhere else. As for the distal face, there is no clear spalling observed. With the plastic strain distribution, a light degree of damage is seen in a wide area surrounding the centre of the slab, but no local damage is observed.

Both charge shape and point of detonation can significantly affect the magnitude and spatial distribution of the pressure and impulse produced by a blast wave, particularly in the near-field. 
This phenomenon has been noted by other researchers as well (Chen et al., 2019; Hao et al., 2016; Langran-Wheeler et al., 2017). Wenzel and Esparza (1974) demonstrated that a cylindrical explosive of Composition B with a diameter-to-thickness ratio of 3.25 can produce normally reflected impulses up to five times greater than those produced by a spherical explosive of the same mass. Similarly, Tancreto (1975) conducted air blast measurements of cylindrical Composition B explosives detonated on the ground surface, and found that the effect of charge shape was still significant up to a scaled distance of $8 \mathrm{~m} / \mathrm{kg}^{1 / 3}$. Sherkar et al. (2016) conducted a numerical investigation on the influence of charge shape, charge orientation, and the point of detonation within the charge on free-field incident overpressures and impulses. Based on these results, it was concluded that the effects of charge shape and point of detonation can be ignored at scaled distances greater than $3 \mathrm{~m} / \mathrm{kg}^{1 / 3}$. However, in the near-field and mid-field, peak overpressure and impulse are significantly affected by charge shape and point of detonation, in comparison to those produced by a spherical charge of the same mass detonated from the centre. Through numerical analysis, Xiao et al. (2020) found that for near-field explosions, neglecting the effect of the cylindrical charge shape can result in an underestimation of peak overpressure by a factor as high 6.6, and maximum impulse by a factor as high as 2.0. It was also found that the shock front generated by cylindrical charges smooths as scaled distance increases, eventually transitioning into hemispherical in the far-field. However, cylindrical charges still generate blast loads approximately 1.2 times higher in the far field than those generated by hemispherical charges of the same mass.

The cylindrical charge mass generates spherical waves in both the radial and axial direction of the charge. The emanating waves constructively interfere as they propagate, forming a reinforced wave of increased magnitude, which depends on charge orientation, detonation point, and aspect 
ratio of the cylinder (Sherkar et al., 2016). The complex interactions of these shock waves have not been fully quantified in the near-field (Langran-Wheeler et al., 2017). As a result of these interactions, the blast pressure produced by the detonation of a cylinder is significantly higher than that of a spherical explosive. Therefore, the amount of damage incurred on the RC slab in Figure 68 is significantly higher when CFD modelling is used to simulate the true detonation of a cylindrical charge.

The results of these additional analyses verify the importance of using CFD for near-field blast events. By allowing for the definition of different charge shapes, CFD is able to model the blast pressures to a significantly higher degree of accuracy in the near-field. This option is not available in the simplified modelling method of LBE. Furthermore, these results also highlight the importance of correct and accurate modelling when using CFD, as shown when the experiment is modelled using CFD but with an incorrect charge shape.

\subsubsection{Validation against Zhang et al. (2013) Test}

\subsubsection{Experiment Details}

The third validation was completed using the experimental data from a study by Zhang et al. (2013). The authors tested a series of RC beams under near-field blast loading. The maximum displacement of the beams was recorded and the degree of local damage due to crushing and spalling was measured. Beam displacement was recorded using a cluster of steel needles that perforated an aluminum plate cover allowing them to only move in the longitudinal direction. The needles were stabbed into a sand-filled barrel. The deflection of the beam caused the needles to depress, which was used to determine the maximum deflection.

For the purposes of this validation, beam B2-4 was selected. It was subjected to $0.75 \mathrm{~kg}$ of TNT at a standoff distance of $400 \mathrm{~mm}$ resulting in a scaled distance of $0.4 \mathrm{~m} / \mathrm{kg}^{1 / 3}$. Figure 69 shows the 
experimental setup. The TNT was shaped as a cylinder and suspended above the beam. The standoff distance was measured from the bottom of the cylinder to the top edge of the beam. The maximum deflection was recorded at the centre of the beam using the steel needles shown. In the experimental setup, the intent was to provide fully fixed supports, constraining the ends of the beam from translation and rotation in all directions. Based on Figure 69, steel angles were used to fix the ends with pieces of wood places between the steel and the RC beam. However, the true fixity of the support is unknown and lies somewhere between pinned and fixed. Even small deformation in the steel angles or movement in the wood could have a significant effect on the level of fixity at the supports. The uniaxial compressive strength of the concrete was $40.45 \mathrm{MPa}$. The reinforcement detailing, shown in Figure 70, consisted of four $6 \mathrm{~mm}$ diameter steel bars used as the longitudinal reinforcement and closed stirrups of $6 \mathrm{~mm}$ diameter spaced at $60 \mathrm{~mm}$. The yield strength and ultimate strength of the steel reinforcement were $395 \mathrm{MPa}$ and $501 \mathrm{MPa}$, respectively.

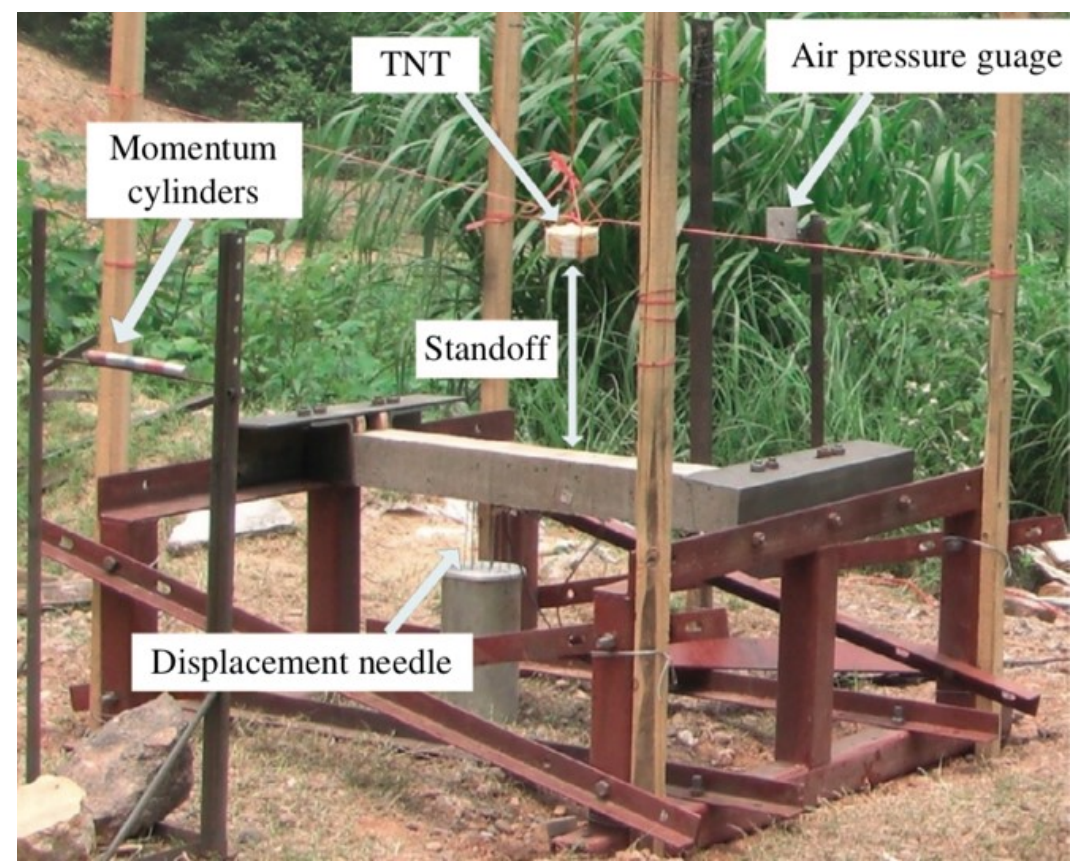

Figure 69: Field experimental setup for testing RC beams (Zhang et al., 2013). 


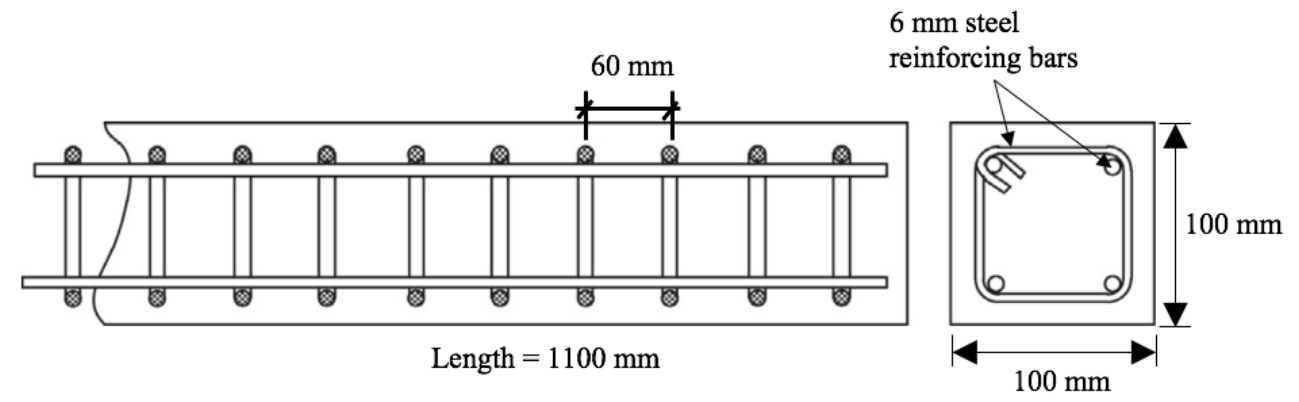

Figure 70: Steel reinforcement detailing of beam B2-4 tested by Zhang et al. (2013).

\subsubsection{FE Model}

This experiment was modelled using the 3D-to-3D mapping technique described in Section 4.2.4. As shown in Figure 71a, the first phase of the FE analysis consisted of 3D modelling of the explosive charge as a 3D cylinder as well as the air box. Modelling the charge as a cylinder has a significant effect on both the magnitude of the pressure in the near-field, but also the spatial variation, as observed in Figure 71b. The air box in the 3D model used a mesh size of $2 \mathrm{~mm}$, for a total of $6,407,469$ solid elements. Similar to the previous validation studies, a quarter symmetric model is used to reduce the number of elements and improve the computational performance of the model.

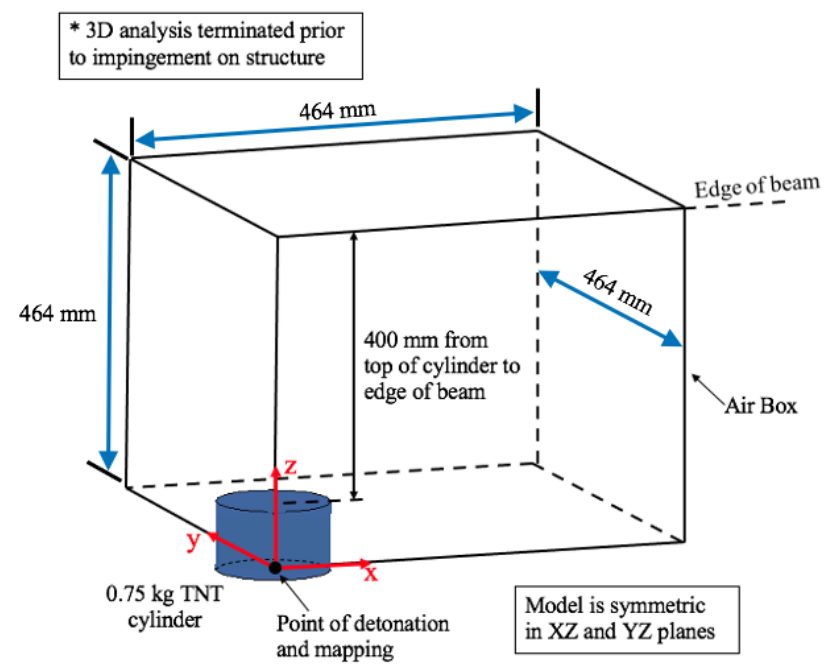

(a)

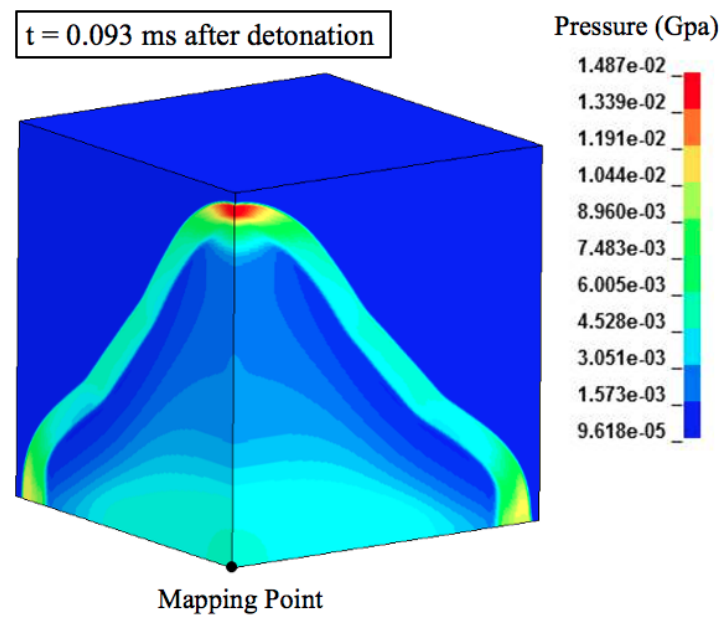

(b)

Figure 71: (a) TNT cylinder is detonated in a 3D model (b) Pressure distribution just prior to mapping. 
Just prior to impingement on the structure, the first phase of the 3D model is terminated, and the data is mapped onto the second phase of the 3D model containing the structure. Figure 72a shows the quarter-symmetric 3D model where the blast wave is allowed to interact with the beam. Because the detonation process was modelled using a fine mesh, it was possible to map it onto a coarser air mesh. The air mesh size in this model was $20 \mathrm{~mm}$, giving a total of 421,875 solid elements. The RC beam, located $400 \mathrm{~mm}$ from the edge of the cylinder, was modelled using a mesh size of $5 \mathrm{~mm}$, for a total of 22,000 solid elements, with the CSCM concrete material model. While a larger mesh size would have sufficed in terms of accurate deflection prediction, a smaller mesh size was selected to refine the resolution of the localized damage. The steel reinforcement was modelled using an elastoplastic material model (see Section 4.1.2.1 for more information) with a mesh size of $10 \mathrm{~mm}$. In order to more accurately reflect the experimental setup, steel plates were modelled for the support conditions using the same material model and mesh size as that used for the reinforcing bars. Nodal constraints preventing translation and rotation in all directions were then applied to the plates and the interaction of the plates and the beam was modelled using CONTACT_AUTOMATIC_SURFACE_TO_SURFACE, as described in Section 4.1.3. Displacements were collected at the centre of the beam. For erosion of concrete, the ERODE parameter was set to 1.2 for this model, which was the same value used for the previous verification study. 


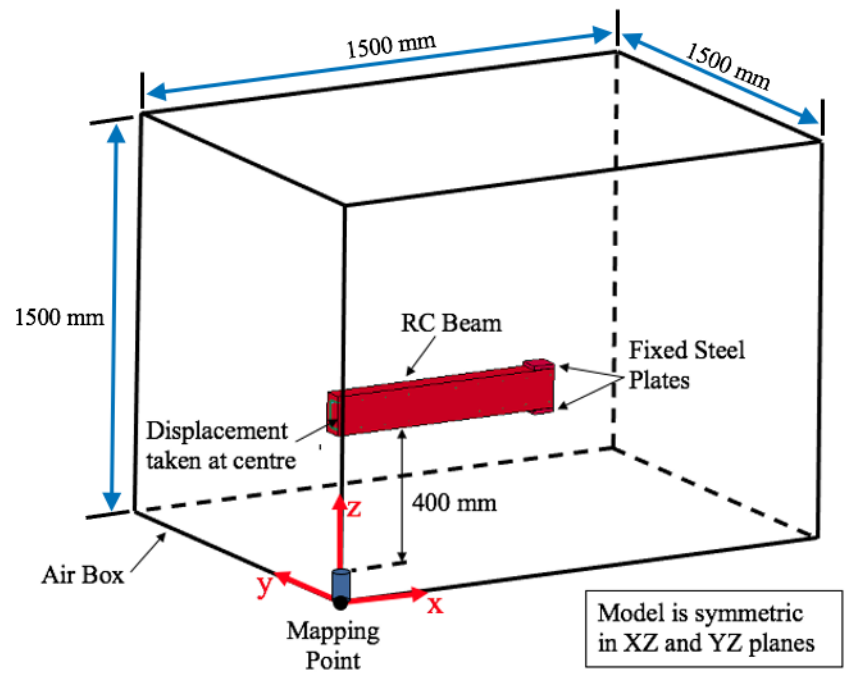

(a)

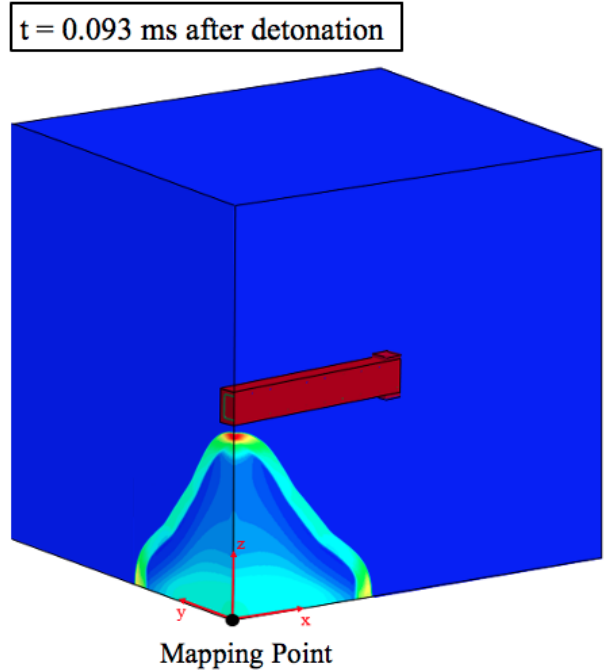

(b)

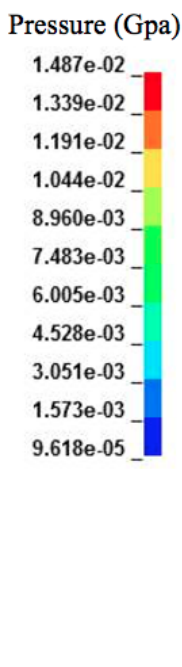

Pressure (Gpa)

$1.191 \mathrm{e}-02$

$1.044 \mathrm{e}-02$

$8.960 \mathrm{e}-03$

$7.483 \mathrm{e}-03$

$6.005 \mathrm{e}-03$

$4.528 \mathrm{e}-03$

3.051e-03

$1.573 \mathrm{e}-03$

$9.618 \mathrm{e}-05$

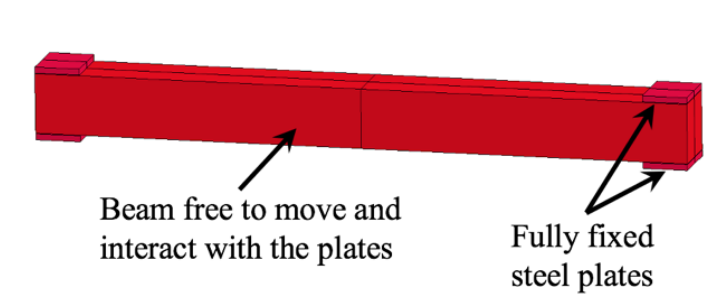

(c)

Figure 72: (a) Depiction of 3D model where blast wave interacts with the beam (b) Mapping of pressures just prior to interaction (c) Support condition of the beam (fixed support).

\subsubsection{Results and Discussion}

The authors recorded a maximum experimental deflection of $40 \mathrm{~mm}$. Figure 73 shows the predicted deflection-time history of the beam with the maximum recorded experimental deflection. The FE model recorded a maximum deflection of $35 \mathrm{~mm}$. While this prediction is deemed acceptable for a blast event, the discrepancy between the experimental and FE model maximum deflection is likely a result of the support conditions. As previously mentioned, the intent was to fully fix the beam ends in the experiment. However, the actual fixity of the supports was unknown but somewhere between pinned and fixed. Uncertainty regarding support conditions has a significant effect on predicted deflections. The steel plates in the FE model were fully fixed and 
therefore underestimated the maximum deflection. A sensitivity analysis on the effect of end support condition on the structural response is provided at the end of this section.

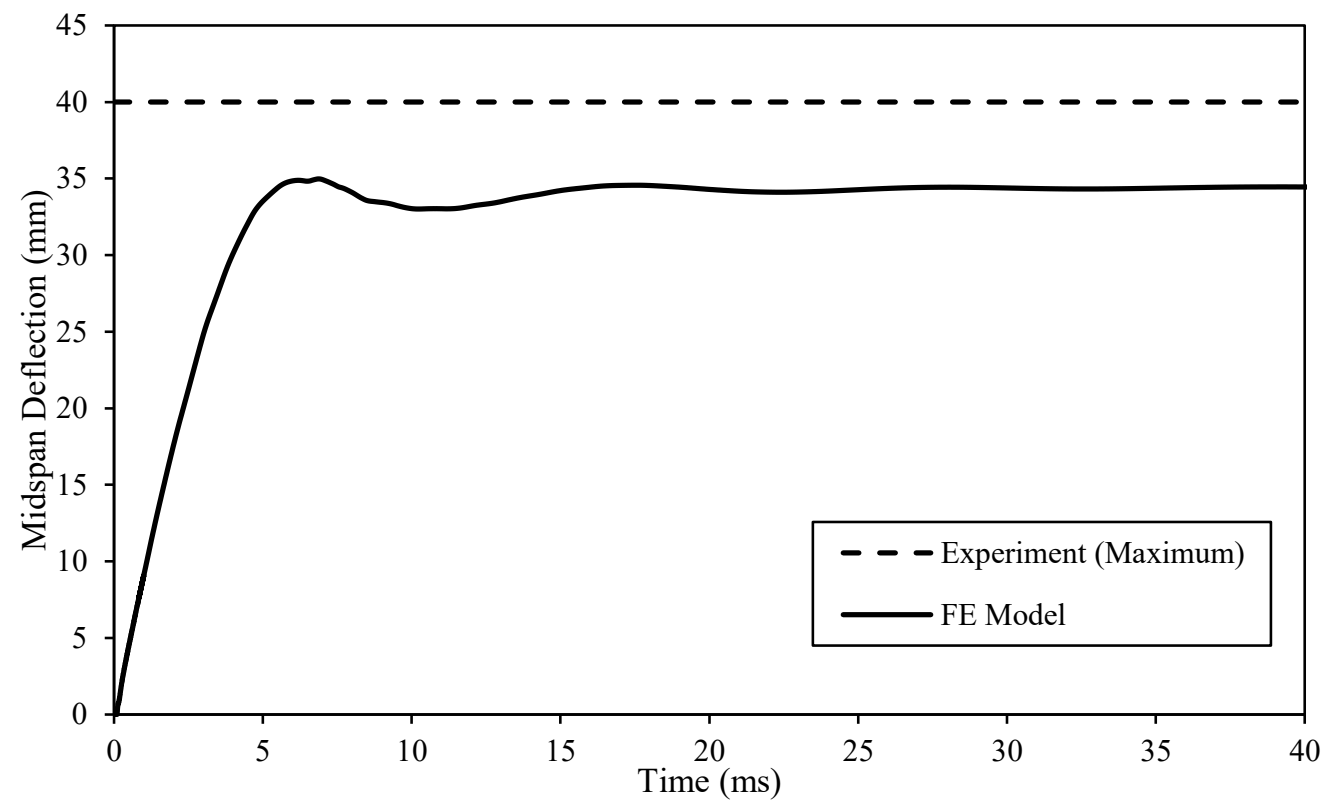

Figure 73: Comparison of the maximum experimental deflection with the predicted deflection by the FE model.

In addition to the measured deflection, the local damage predicted by the FE model was compared to that observed in the experiment. Figure 74 shows the experimental damage of the beam from different perspectives. The authors also reported that severe localized damage was observed with approximately $120 \mathrm{~mm}$ of the front compressional fragmented area and $150 \mathrm{~mm}$ of back spallation. In comparison, the FE model predicted $100 \mathrm{~mm}$ of crushing on the incident face of the beam and $140 \mathrm{~mm}$ of spalling on the back face of the beam. The lengths of damaged areas, which are also presented in Table 10, were measured to the last fully damaged element. Figure 75 shows the localized damage to the beam predicted by the FE model, with a close-up shown in Figure 76 . The FE model was able to predict the damage of the RC beam with good accuracy. 


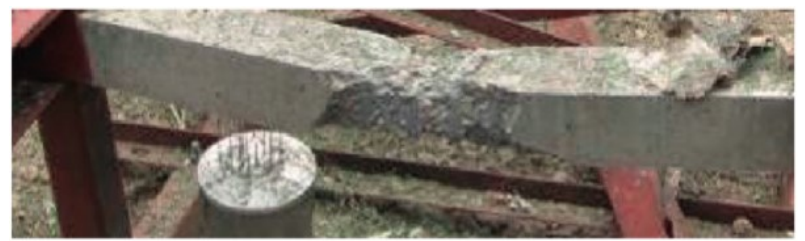

(a)

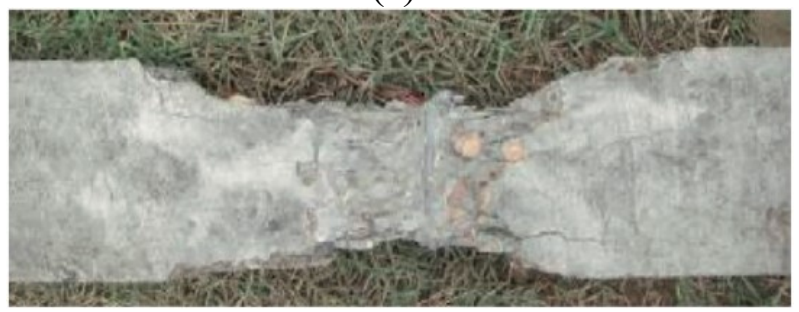

(c)

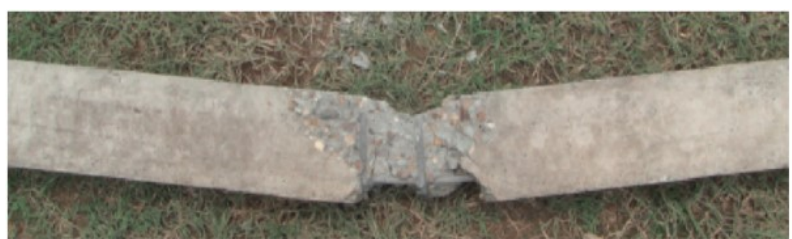

(b)

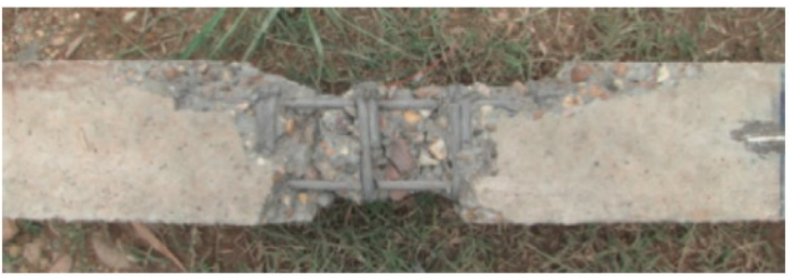

(d)

Figure 74: Damage to beam B2-4 (a) The overall destruction (b) Side face (c) Front face (d) Back face (Zhang et al., 2013).

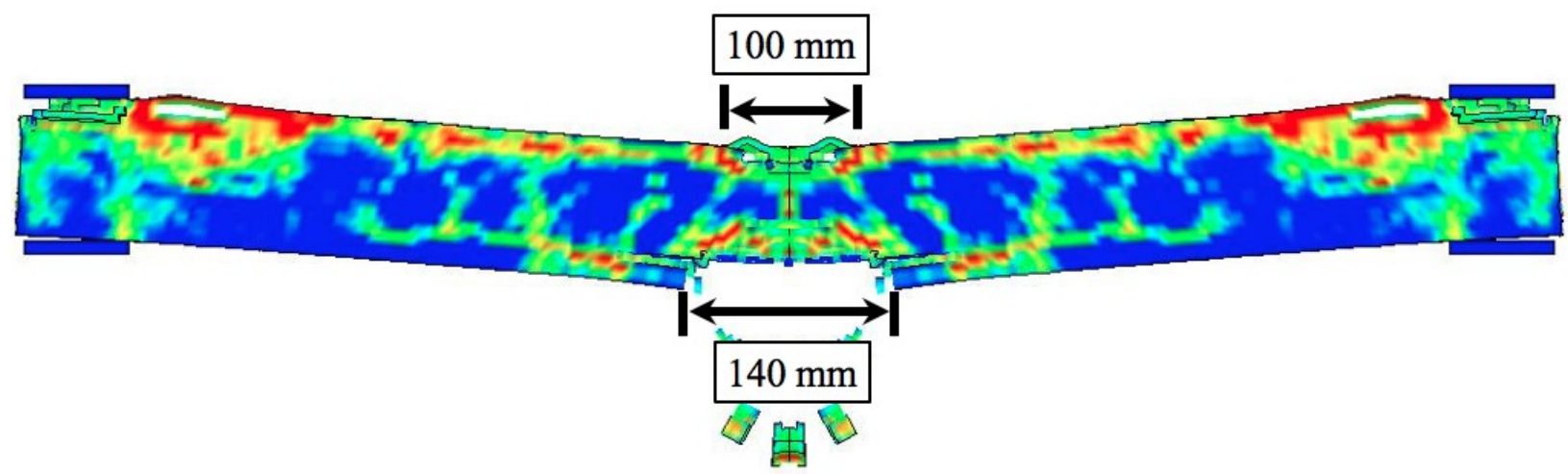

Figure 75: FE model prediction of the deflected shape of the blast-damaged beam.

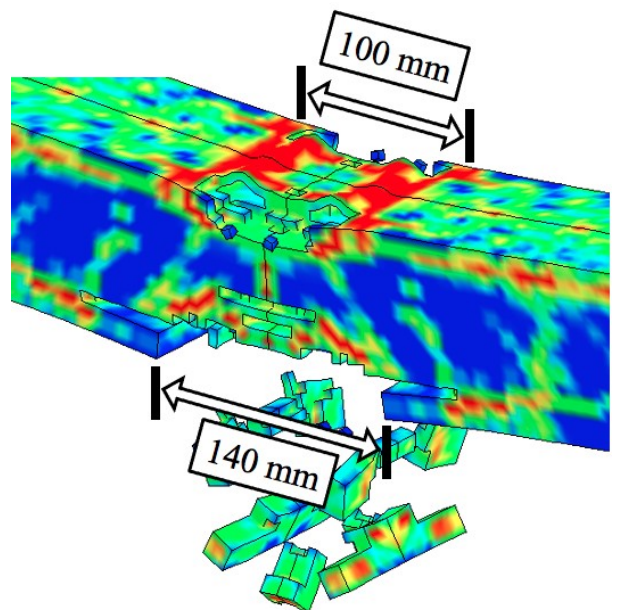

Figure 76: Close-up of the damaged area in the FE model. 
Table 10: Comparison of damage between experiment and FE model.

\begin{tabular}{l|c|c} 
& Experiment & FE Model \\
\hline $\begin{array}{l}\text { Length of crushing on } \\
\text { incident face (top of beam) }\end{array}$ & $120 \mathrm{~mm}$ & $100 \mathrm{~mm}$ \\
\hline $\begin{array}{l}\text { Length of spalling on } \\
\text { distal face (bottom of beam) }\end{array}$ & $150 \mathrm{~mm}$ & $140 \mathrm{~mm}$
\end{tabular}

The effective strain distribution of the beam is presented in Figure 77 and produces a similar damage profile to that presented in Figure 75 . The effective stress distribution of the beam is presented in Figure 78. Under the loading conditions, the DIF for the concrete was computed to be approximately 1.18 , resulting in a dynamic compressive strength of $48 \mathrm{MPa}$. The top portion of the beam at midspan experienced crushing, similar to what was reported in the experiment. As these elements are fully damaged, they have lost all strength and stiffness, and some have been deleted from the simulation.

The axial strain distribution in the steel reinforcement is presented in Figure 79. Since both the yield strength and ultimate strength of the steel were provided in the study, a trilinear stress-strain response was used for the steel reinforcement. Under the loading conditions, the DIF for the steel was computed to be approximately 1.43 , resulting in a dynamic yield strength and strain of 565 MPa and 0.0028, respectively. Although the condition of the steel was not reported in the study, the FE model computed that a significant portion of the bottom layer longitudinal steel had yielded at the midspan (see Figure 79a). The maximum strains in the steel reinforcement are shown in Figure 79b. As the magnitude of the strains are significantly lower than typical rupture strains for steel reinforcement (around $100-150$ millistrain), it was concluded that no rupturing of steel reinforcement occurred. 


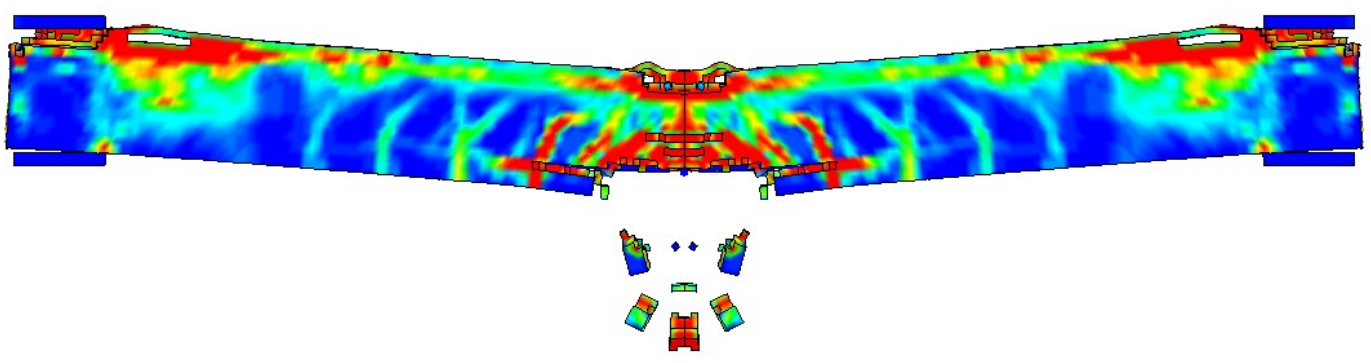

$3.500 \mathrm{e}-02$

$3.150 \mathrm{e}-02$

$2.800 \mathrm{e}-02$

$2.450 \mathrm{e}-02$

$2.100 \mathrm{e}-02$

$1.750 \mathrm{e}-02$

$1.400 \mathrm{e}-02$

$1.050 \mathrm{e}-02$

$7.000 \mathrm{e}-03$

$3.500 \mathrm{e}-03$

$0.000 e+00$

Figure 77: Effective strain distribution of the blast-damaged beam.

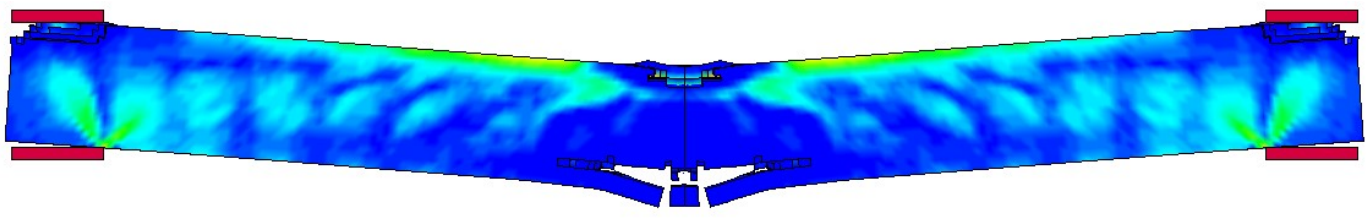

Effective Stress

$$
\text { (Gpa) }
$$

$4.770 \mathrm{e}-02$

$4.293 \mathrm{e}-02$

$3.816 \mathrm{e}-02$

$3.339 \mathrm{e}-02$

$2.862 \mathrm{e}-02$

$2.385 \mathrm{e}-02$

$1.908 \mathrm{e}-02$

$1.431 \mathrm{e}-02$

$9.540 \mathrm{e}-03$

$4.770 \mathrm{e}-03$

$0.000 \mathrm{e}+00$

Figure 78: Effective stress distribution of the beam at peak response.

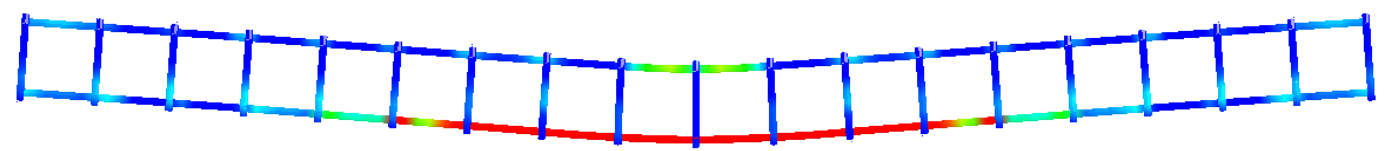

(a)

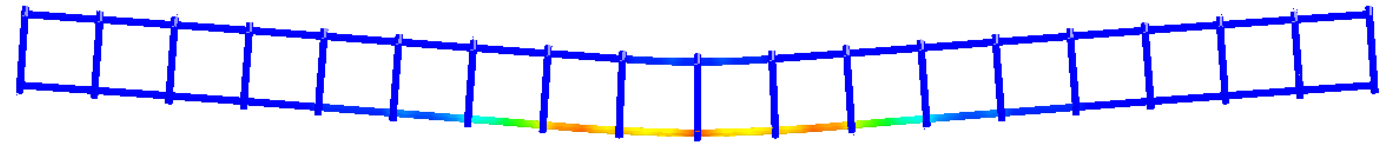

(b)

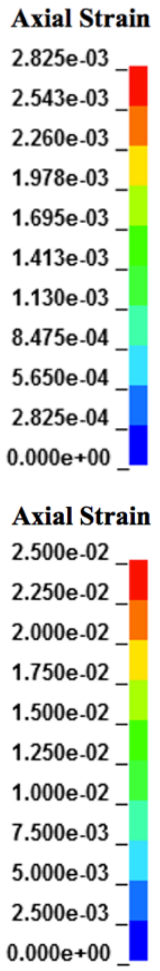

Figure 79: Axial strain distribution in steel reinforcement showing (a) dynamic yield strain and (b) maximum strains. 
As previously mentioned, modelling support conditions was an important parameter in order to accurately simulate the response of the structure. However, it was also an uncertainty in the experimental test. In order to highlight the significant effect of the support conditions, the beam was also modelled using fixed nodal constraints and a pinned support condition. Figure 80 shows the deflection of the beam when the end support conditions are represented by modelling the end steel plates, using fixed nodal constraints, or using pinned supports. The experimental maximum deflection is also plotted for comparison. The fixed nodal constraints significantly underestimate the deflection with a $73 \%$ difference in maximum deflection in comparison to the experiment. In contrast, the pinned supports significantly overestimate the deflection with a $60 \%$ difference in maximum deflection in comparison to the experiment. Explicitly modelling the end steel plates provides the best estimation of deflection with a $12.5 \%$ difference in maximum deflection in comparison to the experiment. This modelling option provides the most realistic simulation of the experimental setup, allowing for the beam to move freely and interact with the fully fixed steel plates. The deflected shapes of the beams are presented in Figure 81 with the plastic strain distribution. The model with fixed nodal constraints shows very little deformation in the beam while the model with pinned supports shows facture at the beam midspan. 


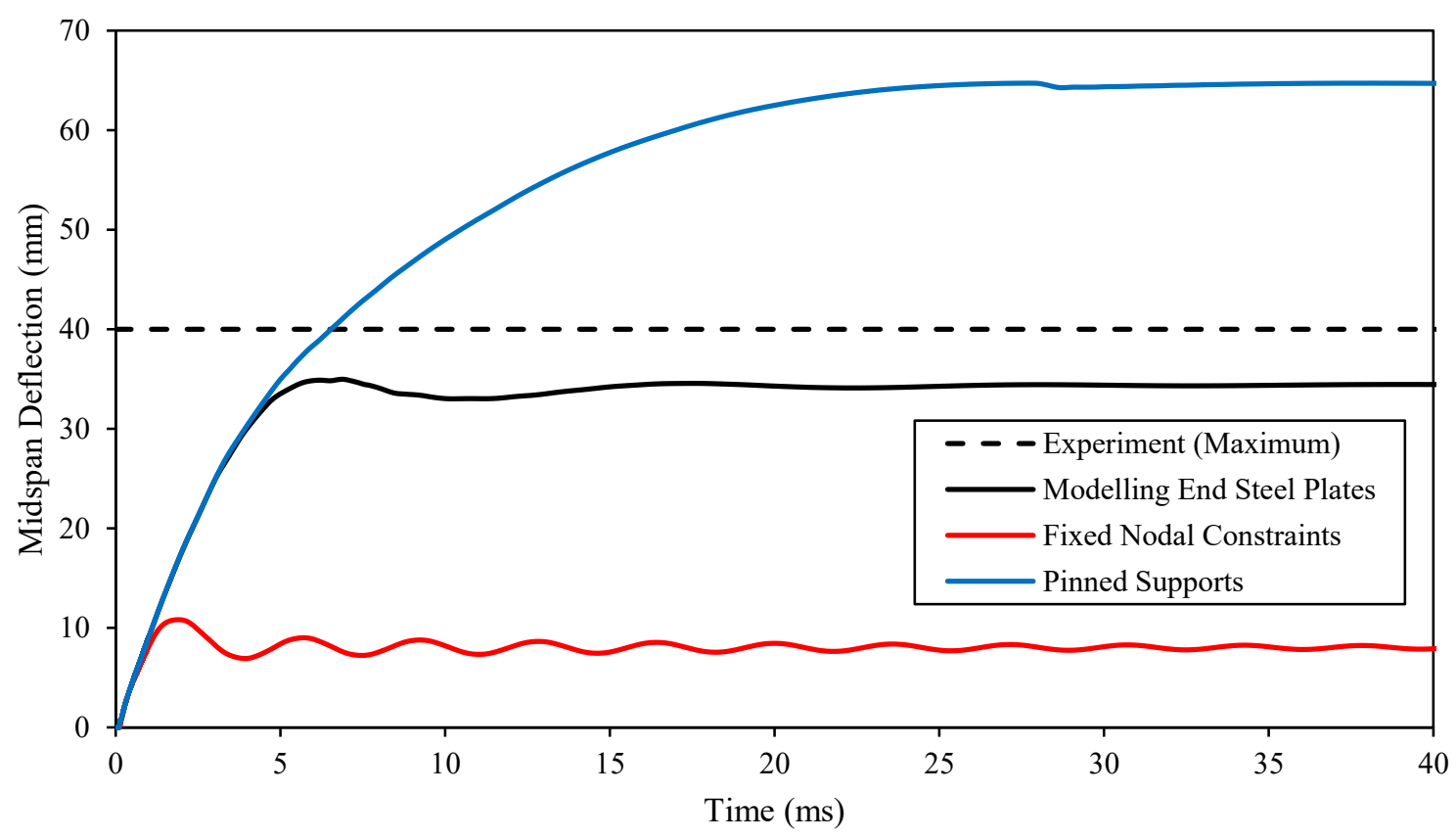

Figure 80: Effect of different support conditions on the displacement of the beam.

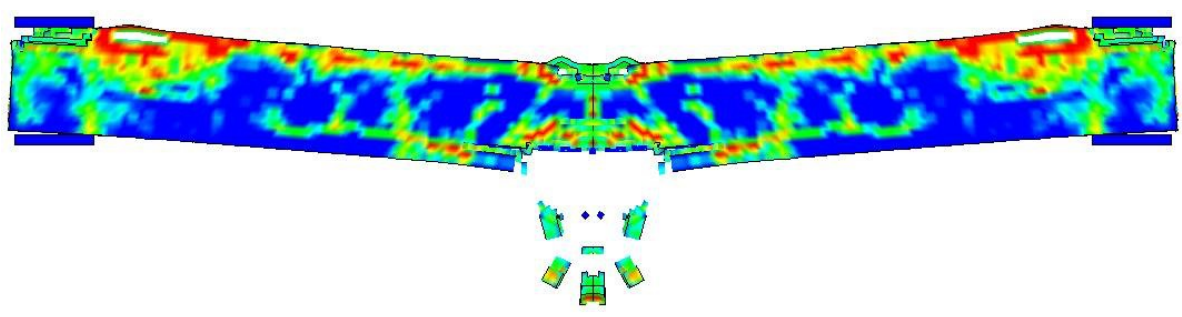

Plastic Strain

(a) Modelling end steel plates

$9.990 \mathrm{e}-01$

$8.991 \mathrm{e}-01$

$7.992 \mathrm{e}-01$

$6.993 \mathrm{e}-01$

$5.994 \mathrm{e}-01$

4.995e-01

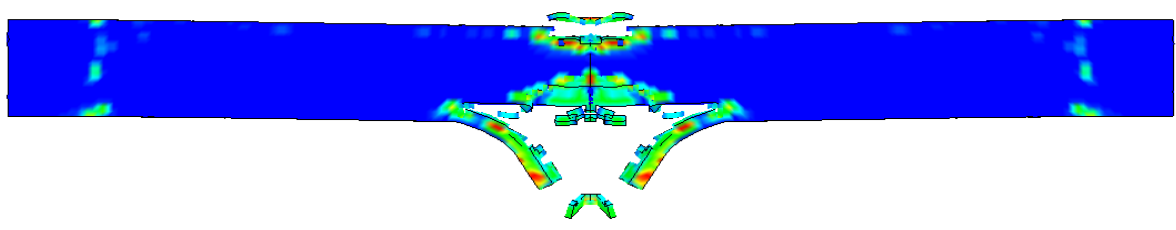

$3.996 \mathrm{e}-01$

2.997e-01

$1.998 \mathrm{e}-01$

(b) Fixed Nodal Constraints

$9.990 \mathrm{e}-02$

$0.000 \mathrm{e}+00$

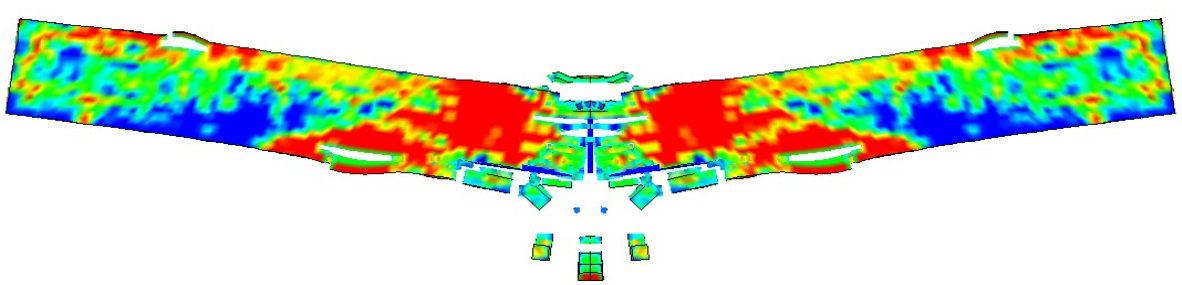

(c) Pinned Support Condition

Figure 81: Deflected shape of blast-damaged beam using (a) fixed steel supports, (b) fixed nodal constraints, (c) pinned support condition. 
Similar to the previous validation study, the beam used in the experiment by Zhang et al. (2013) was also modelled using the LBE modelling method and the CFD modelling method with a spherical charge. Figure 82 presents the beam deflection-time histories as predicted by the different models. It is apparent that the LBE model and the CFD model using a spherical charge both severely underestimate the deflection of the beam. However, based on the discussion in Section 5.2.2.3 regarding charge shapes this discrepancy is logical and expected.

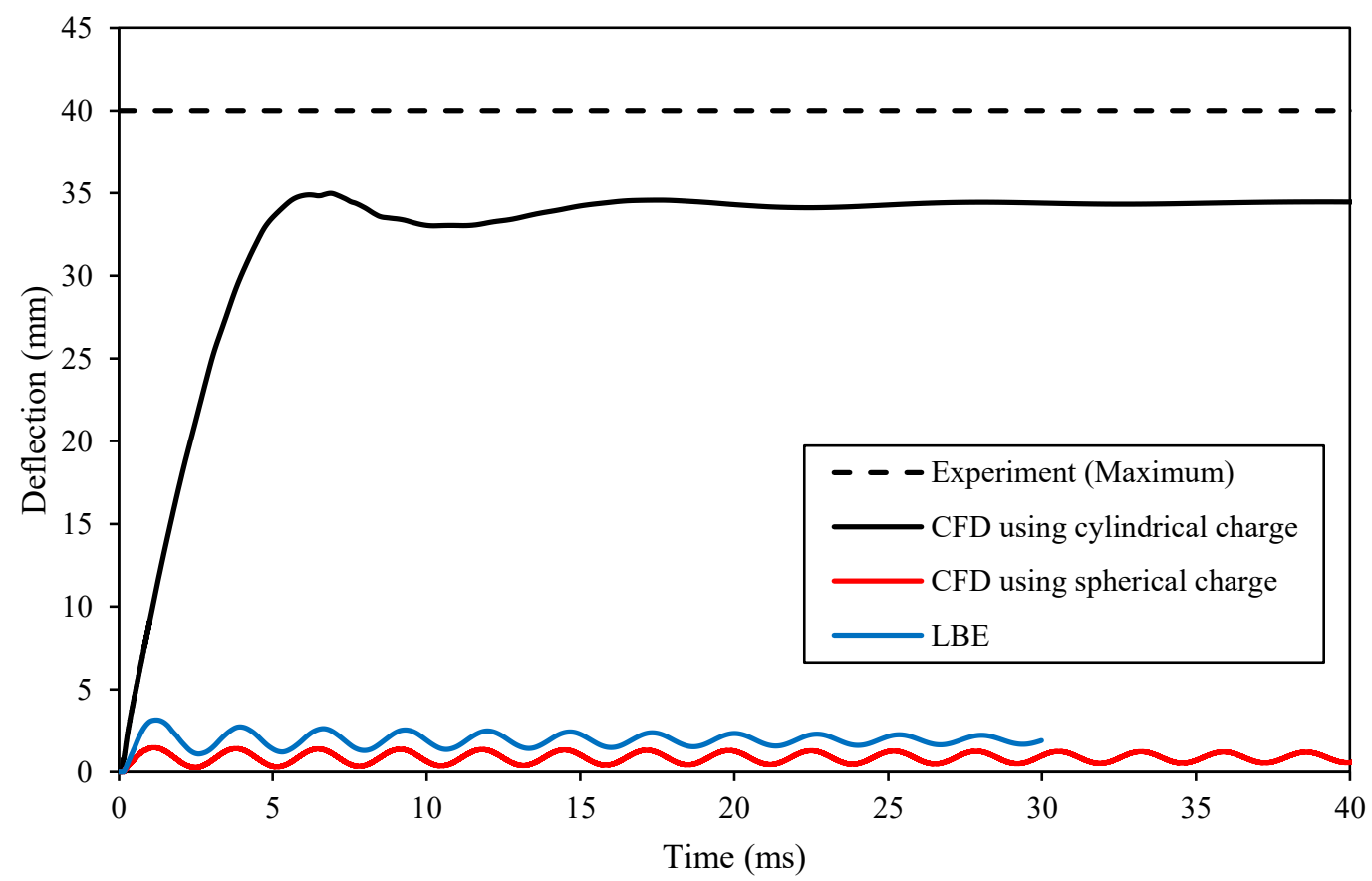

Figure 82: Beam deflections predicted by different FE models.

Figure 83 shows the beam damage profiles of the different models. Neither the LBE model nor the CFD model with the spherical charge are able to capture the significant degree of local damage that occurs at the centre of the beam. It is again proven that the shape of the explosive has a significant influence on blast loadings in the near-field. LBE does not have the option to model different charge shapes and therefore is not able to accurately predict the blast loads in the nearfield nor the response of the structure. Furthermore, the importance of accurate CFD modelling for near-field blast loading is also demonstrated. 


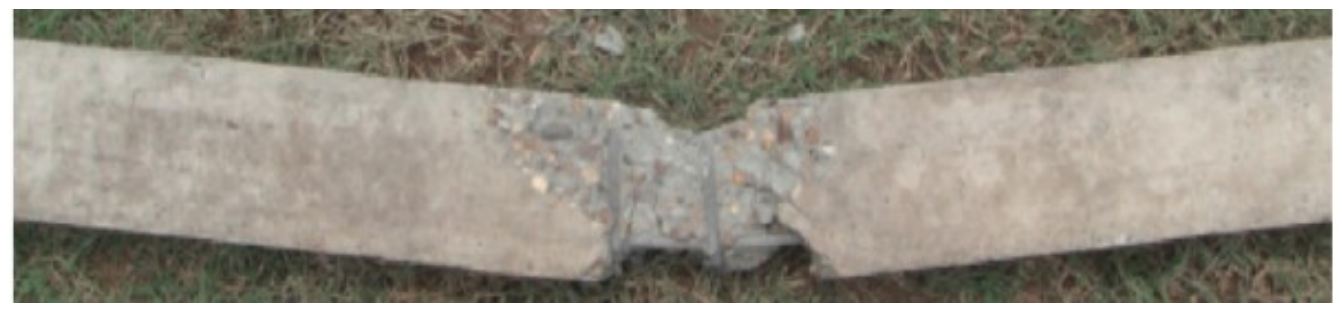

(a) Experiment

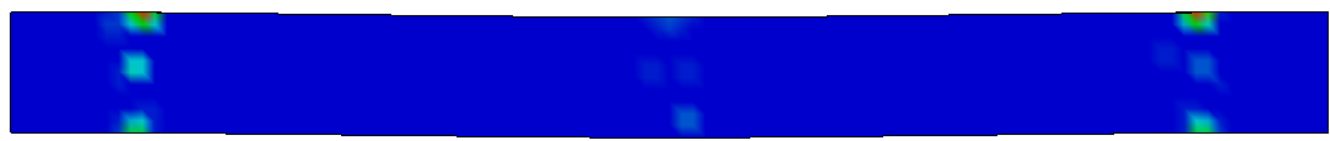

Plastic Strain

(b) LBE modelling method

$9.990 \mathrm{e}-01$

$8.991 \mathrm{e}-01$

$7.992 \mathrm{e}-01$

$6.993 \mathrm{e}-01$

$5.994 \mathrm{e}-01$

4.995e-01

(c) CFD modelling method with spherical charge

$3.996 \mathrm{e}-01$

2.997e-01

$1.998 \mathrm{e}-01$

$9.990 \mathrm{e}-02$

$0.000 \mathrm{e}+00$

$$
\text { (1..) }
$$

(d) CFD modelling method with cylindrical charge

Figure 83: (a) Beam damage recorded in experiment (b) Beam damage computed by LBE (c) Beam damage computed by CFD with spherical charge (d) Beam damage computed by CFD with cylindrical charge. 


\section{Parametric Study}

\subsection{Numerical Model}

Due to the limited experimental data in blast, there is a need for analytical studies that investigate blast resistance of structures. FE modelling provides a more cost-effective alternative to experimental work involving blast loads, which can be very expensive. Using the validated FE model, a series of numerical simulations were conducted to examine the effect of a number of design parameters on the performance of RC columns under near-field blast loads. The design parameters selected for this parametric study were: spacing of transverse reinforcement, concrete cover, axial load ratio, and cross-section shape. These design parameters were selected as it is expected that they will have a significant influence on column damage and consequently the residual capacity. The effect of the design parameters was evaluated using a number of performance measures such as maximum and residual displacement at mid-height of the column, length of the damage zone, and residual axial capacity. Stresses in the concrete and steel reinforcement were also analyzed to evaluate damage modes. Failure of a key load-bearing element, such as a column, can result in the progressive collapse of the entire structure. Thus, evaluating the influence of key design parameters on the blast performance of RC columns is critical. While previous studies have investigated similar parameters, they were typically conducted using simplified empirical methods of analysis. As demonstrated in the validation of the numerical model, these empirical methods are not able to accurately model blast loadings in the near-field or structural response.

Two loading cases were analyzed in the numerical simulations. In order to simulate a hand-held explosive, a charge mass of $50 \mathrm{~kg}$ of TNT was selected as the explosive in the first loading case. The explosive was detonated in the near-field with a scaled distance of $\mathrm{z}=0.25 \mathrm{~m} / \mathrm{kg}^{1 / 3}$ 
(corresponding to a distance of approximately $920 \mathrm{~mm}$ ) at mid-height of the column. A second set of analyses was also conducted to evaluate the response of the columns to a larger charge mass. For the second loading case, a charge mass of $100 \mathrm{~kg}$ of TNT was detonated at mid-height of the column at a stand-off distance of $1160 \mathrm{~mm}$. This loading case also resulted in a scaled distance of $\mathrm{z}=0.25 \mathrm{~m} / \mathrm{kg}^{1 / 3}$.

Figure 84 shows the detailing of the reference column and its position with respect to the explosive charge. A $300 \times 300 \mathrm{~mm}$ column was selected with a length of $3 \mathrm{~m}$. The column was designed in accordance with CSA A23.3-19 and is intended to represent a ground-storey column of a low- to mid-rise building located in a low-seismic region. The column had four $25 \mathrm{M}$ bars for longitudinal reinforcement, corresponding to a reinforcement ratio of $2.22 \%$. The transverse reinforcement of the column consisted of $10 \mathrm{M}$ ties with a spacing of $150 \mathrm{~mm}$, corresponding to a reinforcement ratio of $0.44 \%$. A concrete cover of $30 \mathrm{~mm}$ was provided based on an exposure class of $\mathrm{N}$ indicating concrete that is not exposed to chlorides nor freezing and thawing.

Using nodal constraints, the column was fixed at the top and bottom ends against translational and rotational movements in all directions. However, in order to transmit axial loads, the top support was allowed to have translational movement in the vertical direction.

An axial load of $844 \mathrm{kN}$ was applied to the reference column, which is approximately equal to $30 \%$ of the factored axial load capacity of the column. This axial load level was selected in order to simulate the level of axial loading that can be expected in ground storey column in a low to midrise structure.

The RC column was built from normal strength concrete with an unconfined compressive strength of $40 \mathrm{MPa}$ and a mass density of $2350 \mathrm{~kg} / \mathrm{m}^{3}$. The reinforcing steel was modelled using a trilinear stress-strain response with a yield strength of $400 \mathrm{MPa}$ and ultimate strength of $600 \mathrm{MPa}$. Young's 
modulus for the steel was $200 \mathrm{GPa}$ and Poisson's ratio was set to 0.3 . For erosion of concrete, the ERODE parameter was set to 1.4 for the simulations in the parametric study.

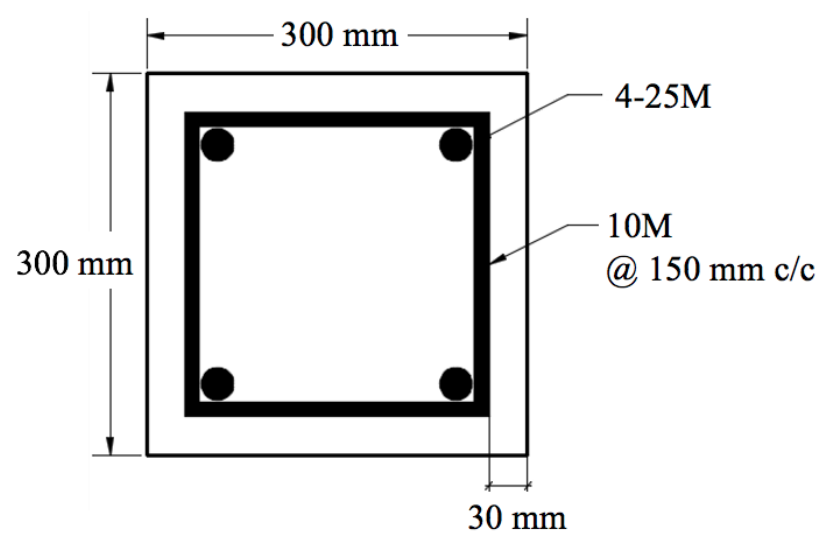

(a)

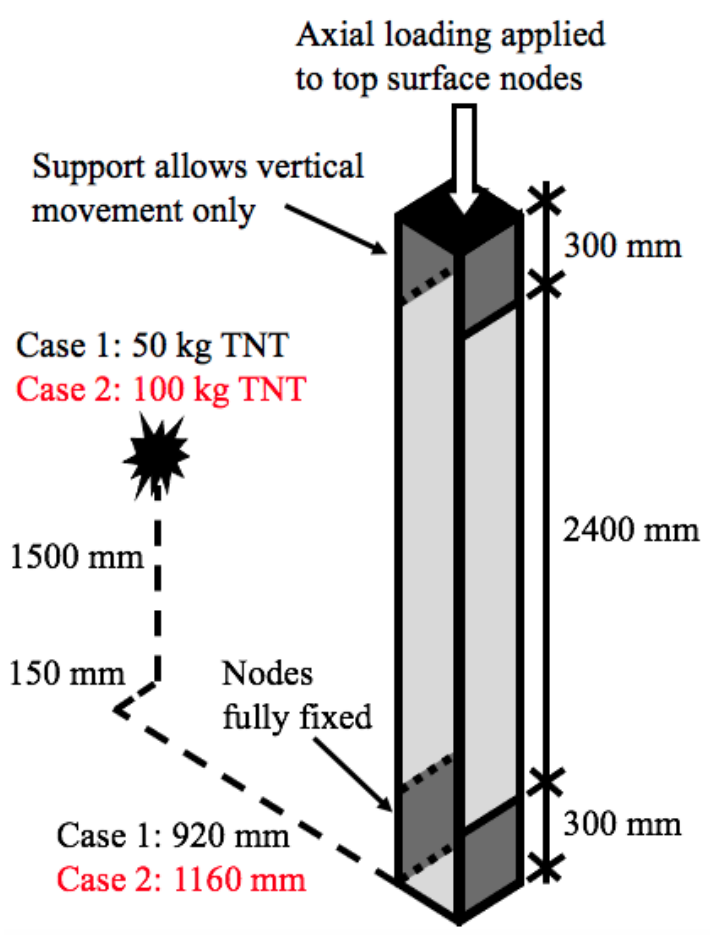

(b)

Figure 84: (a) Cross-section details of reference column (b) Positioning of reference column relative to explosive charge.

\subsection{Results and Discussion}

\subsubsection{Effect of Transverse Reinforcement}

Figure 85 shows the effect of transverse reinforcement spacing on maximum and residual lateral displacements of the columns subjected to $50 \mathrm{~kg}$ and $100 \mathrm{~kg}$ of TNT. For both loading cases, the maximum and residual lateral displacements show similar trends to one another.

For the $50 \mathrm{~kg}$ TNT loading case, there is a significant change in slope at around $150 \mathrm{~mm}$ spacing which is the spacing equal to half the column cross-section dimension. For columns with transverse reinforcement spacing greater than $150 \mathrm{~mm}$, reducing the reinforcement spacing is highly effective in controlling the maximum and residual lateral displacements. However, reducing the spacing of transverse reinforcement to less than $150 \mathrm{~mm}$ is observed to have a negligible effect on the lateral 
displacement. This finding is aligned with the design practice where half of the cross-section dimension is typically considered to be a proper estimate for the spacing of transverse reinforcement in RC columns.

For the $100 \mathrm{~kg}$ TNT loading case, the columns experienced significantly higher lateral displacements, approximately twice the displacements recorded in the $50 \mathrm{~kg}$ TNT case. The effect of the transverse reinforcement spacing is not as apparent as observed in the results of the $50 \mathrm{~kg}$ TNT case. However, the maximum and residual lateral displacements for the columns with transverse reinforcement spacing between $50-150 \mathrm{~mm}$ remain relatively constant. A slight increase in lateral displacements is observed in the columns with $200 \mathrm{~mm}$ and $300 \mathrm{~mm}$ transverse reinforcement spacing. This is consistent with the results of the $50 \mathrm{~kg}$ TNT case. However, due to the increased amount of charge, the columns experience significantly larger displacements, and therefore this trend is not as well-defined.

Other studies conducted at higher scaled distances (greater than $0.5 \mathrm{~m} / \mathrm{kg}^{1 / 3}$ ) also found that increasing transverse reinforcement ratio improved column performance in terms of maximum lateral displacement (Bao \& Li, 2010; Rajkumar et al., 2020). However, the present study shows that reducing transverse reinforcement spacing is effective at controlling both maximum and residual lateral displacements at near-contact scaled distances as well. The effect of transverse reinforcement spacing is further investigated using other performance measures in the following discussion. 


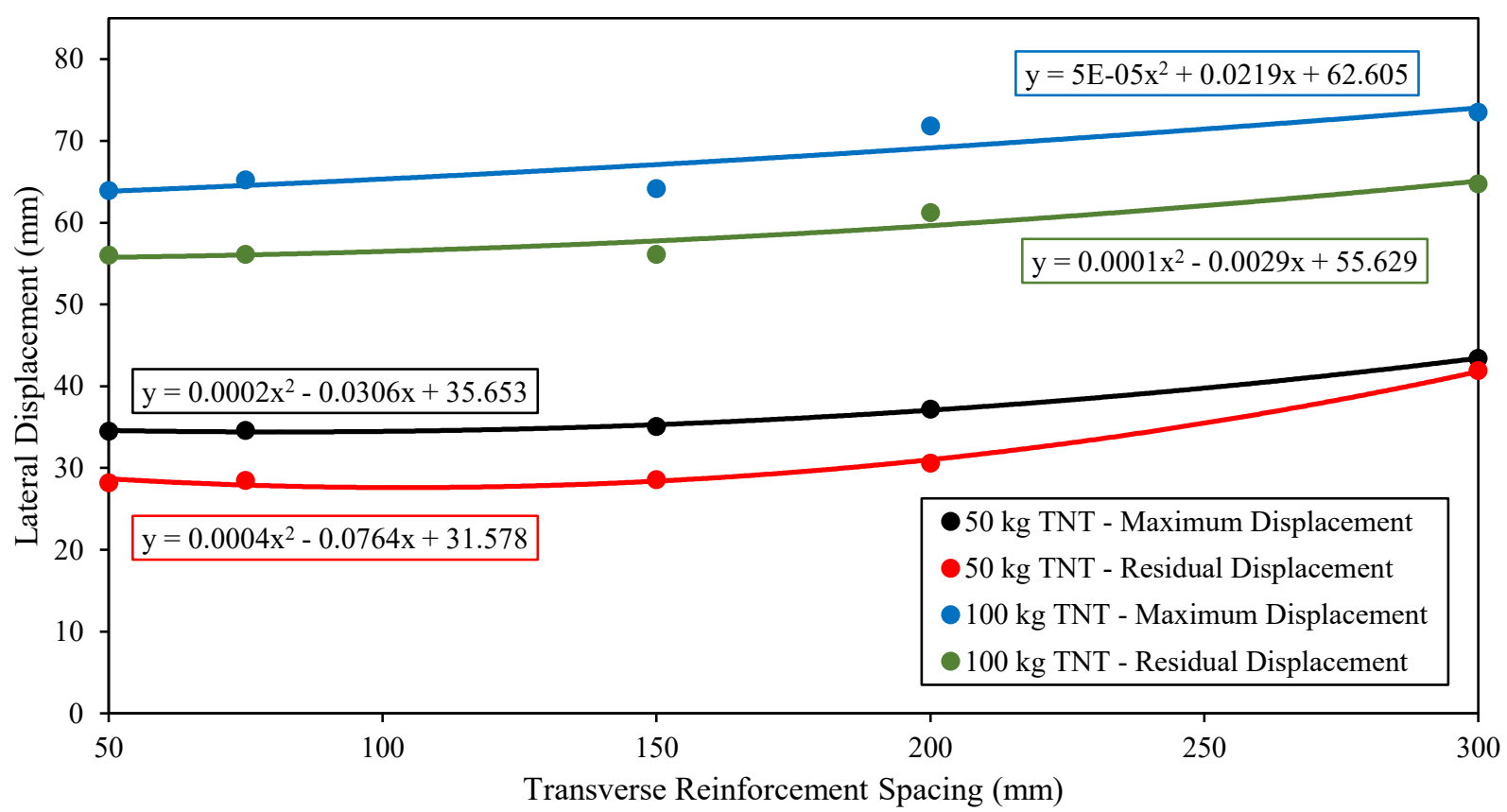

Figure 85: Effect of transverse reinforcement spacing on lateral displacement.

Table 11 shows the distribution of axial strain in the steel reinforcement at peak response. Under both the loading conditions, the DIF for the steel reinforcement was calculated to be approximately 1.4 , resulting in a dynamic yield strength and strain of $560 \mathrm{MPa}$ and 0.0028 , respectively. All the columns showed yielding of longitudinal reinforcement at mid-height and at the supports. For both the $50 \mathrm{~kg}$ and $100 \mathrm{~kg}$ TNT loading cases, as the transverse reinforcement spacing increases, the length of yielded longitudinal steel elements also increases, but the magnitude of the strains decreases. In columns with lower transverse reinforcement spacings, such as $50 \mathrm{~mm}$ and $75 \mathrm{~mm}$, the yielded longitudinal steel of the distal face is localized to the mid-height regions of the columns. There is little variation in axial strain distribution between columns with $50 \mathrm{~mm}$ and 75 $\mathrm{mm}$ transverse reinforcement spacing. This is consistent with the earlier observations in lateral displacement. Columns with spacing of $150-300 \mathrm{~mm}$ show yielding in some transverse reinforcement steel elements, whereas this is not the case for columns with spacing of $50 \mathrm{~mm}$ and $75 \mathrm{~mm}$. Rupture in steel reinforcement typically occurs at strains greater than 100 millistrain. As the strains in the steel were significantly lower, no rupture occurred in any of the columns. 
Table 11: Axial strain distribution in steel reinforcement at peak response for varying amount of transverse reinforcement spacing.

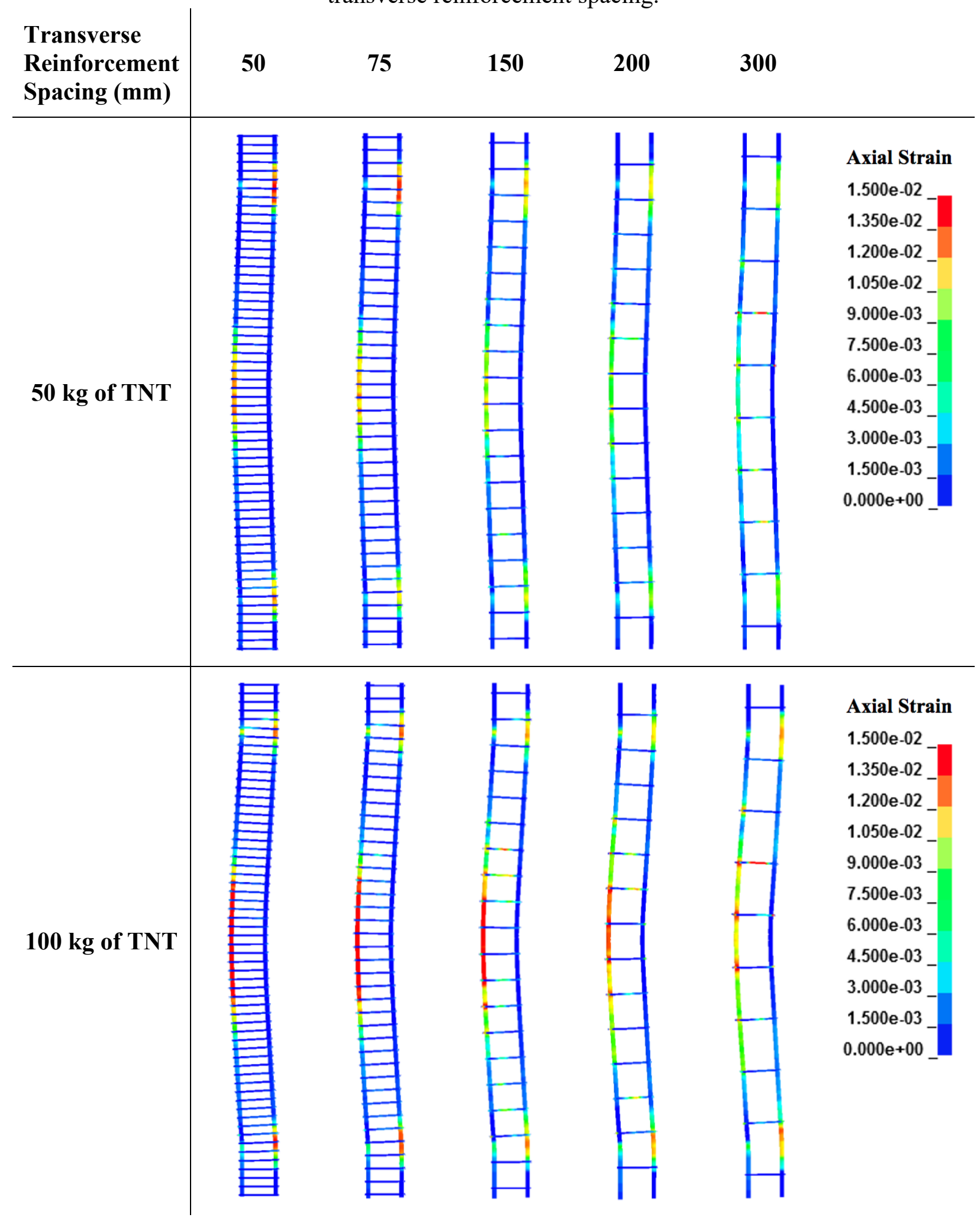


The columns with $150-300 \mathrm{~mm}$ transverse reinforcement spacing likely experienced a flexuralshear response, while the response of the other columns was mainly governed by flexural behaviour. The longitudinal steel in these columns yielded, but they also showed signs of shear distress. This is evident in the yielding of the transverse steel at various points along the column as well as the effective strain distribution in the columns, as shown in Table 12.

For the $50 \mathrm{~kg}$ TNT case in Table 12, columns with transverse reinforcement spacing between 150 $-300 \mathrm{~mm}$ show signs of shear distress near the base support. In the column with $150 \mathrm{~mm}$ spacing, a green diagonal line extends from the edge of the base support at the distal face to mid-height of the column at the incident face. This diagonal line of high strain increases in severity as the transverse reinforcement spacing increases. While the concrete material model used in LS-DYNA is not capable of showing crack propagation, this area of high strain likely represents a large diagonal shear crack since the strain values are significantly larger than the cracking strain of concrete $\left(\varepsilon_{c r} \cong 0.1 \times 10^{-3}\right)$. As the spacing of the transverse reinforcement reduces, the diagonal high strain zone fades away and the shear effects become less important on the structural response of the column. The column with $300 \mathrm{~mm}$ transverse reinforcement spacing has the most severe diagonal shear crack. This observation is consistent with the lateral displacement results in Figure 85 , as this column showed the least amount of displacement recovery. A similar response is observed in the columns subjected to $100 \mathrm{~kg}$ of TNT. Columns with transverse reinforcement spacing between $150-300 \mathrm{~mm}$ also showed similar signs of shear distress at the base support of the columns.

All the columns show areas of high strain extending throughout the depth at mid-height of the column, potentially indicating flexural cracking. These flexural cracks are perpendicular to the longitudinal axis of the column and extend between two extremely high strain zones at the 
midspan, the outermost tensile layer at the distal face and the outermost compressive layer at the incident face. For both the $50 \mathrm{~kg}$ and $100 \mathrm{~kg}$ TNT loading cases, the severity of these cracks increases significantly for the columns with $200 \mathrm{~mm}$ and $300 \mathrm{~mm}$ transverse reinforcement spacing, likely also contributing to the increased lateral displacements of these columns. The strain distribution for columns with spacing between $50-150 \mathrm{~mm}$ is similar when comparing the results of the $50 \mathrm{~kg}$ and $100 \mathrm{~kg}$ TNT loading cases. However, the strain distribution in the columns with $200 \mathrm{~mm}$ and $300 \mathrm{~mm}$ spacing is significantly more severe for the $100 \mathrm{~kg}$ TNT test than that observed in the $50 \mathrm{~kg}$ TNT case. This further supports the conclusion that the reduction of transverse reinforcement spacing less than $150 \mathrm{~mm}$ (ie. half the column cross-section dimension) has a minimal effect on the response of the columns. 
Table 12: Residual effective strain distribution in blast-damaged columns for varying amount of transverse reinforcement spacing.

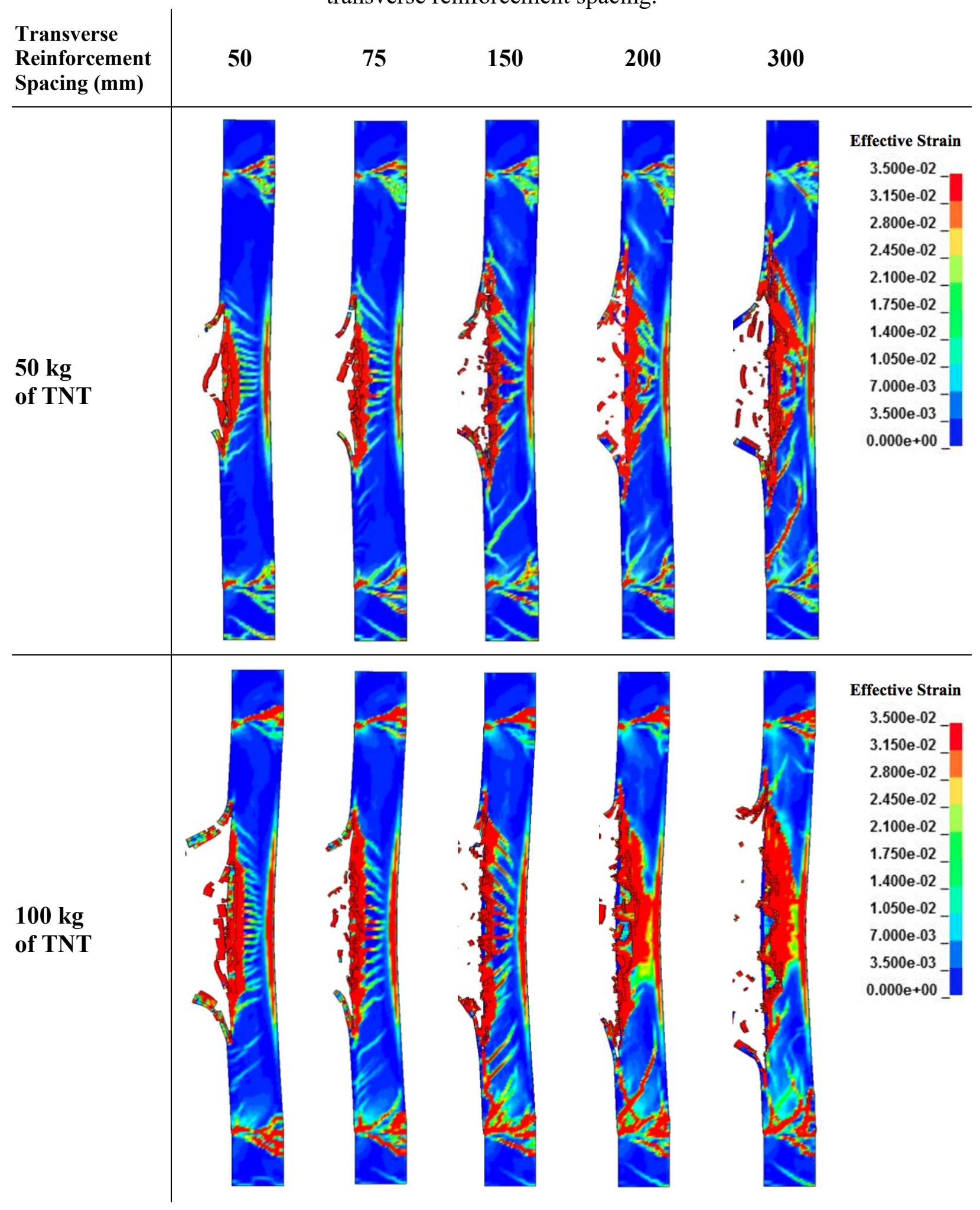


Because of the close stand-off distance of the blast, a certain degree of local damage was seen in all the columns. However, a reduction in transverse reinforcement spacing reduced the local damage to the columns. Figure 86 shows a linear trend between the length of spalling on the distal face of the column and the transverse reinforcement spacing. Spalling was measured on the distal face of the column in the vertical direction by measuring the length of continuously fully damaged elements. As transverse reinforcement spacing decreased, so did the length of spalling on the distal face of the column. The densely spaced transverse reinforcement improved the strength and ductility of the core concrete resulting in a reduction of length of spalling. This trend was observed for both the $50 \mathrm{~kg}$ and $100 \mathrm{~kg}$ TNT loading cases. However, the reduction in length of spalling is more pronounced in the $50 \mathrm{~kg}$ TNT case, with an increased slope in the trendline.

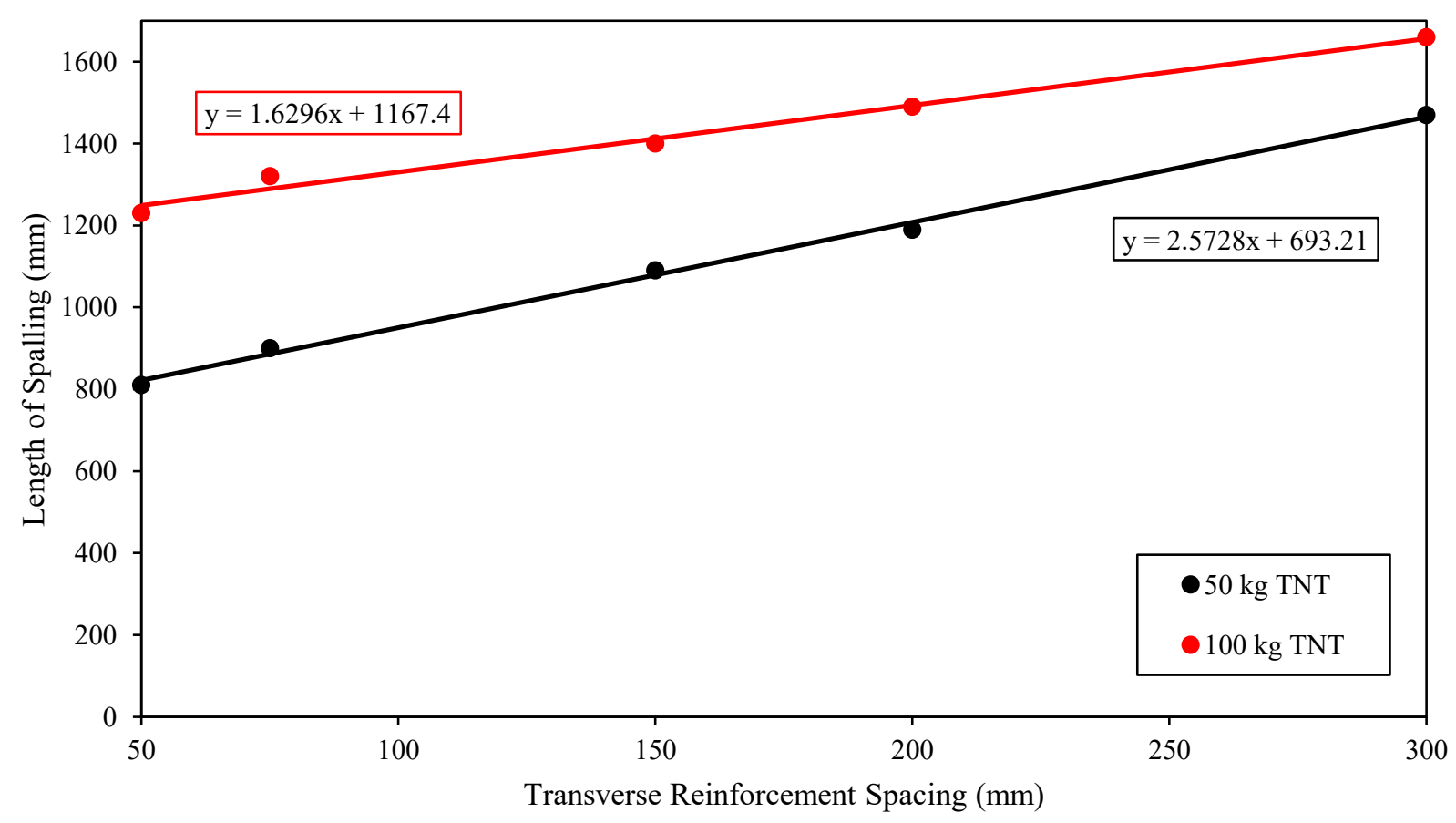

Figure 86: Effect of transverse reinforcement spacing on length of spalling.

Table 13 shows the effective stress distribution in the columns at approximately $6 \mathrm{~ms}$ after detonation of the charge. This is slightly after the peak response of the columns, which typically occurs at approximately $5 \mathrm{~ms}$. The columns presented in Table 13 show a section cut midway 
through the width of the columns, as shown in Figure 87, so that the stresses within the core can be observed. In Table 13, the areas of zero stress (blue areas) indicate spalled elements in tensile regions on the distal face of the columns. The columns exceed the unconfined compressive strength of concrete due to a combination of confinement and strain rate effects. Under both the loading conditions, the DIF for the concrete was computed to be approximately 2.0 , resulting in a dynamic compressive strength of $80 \mathrm{MPa}$. Additionally, Table 14 lists the effect of confinement on the compressive strength, compressive strain, and axial capacity of columns for varying transverse reinforcement spacing. The stresses and strains of confined concrete were computed based on the work of Mander et al. (1988).

For both the $50 \mathrm{~kg}$ and $100 \mathrm{~kg}$ TNT loading cases, columns with transverse reinforcement spacing between $50-150 \mathrm{~mm}$, show similar distributions of compressive stress. Elements experiencing high compressive stresses greater than $\mathrm{f}_{\mathrm{c}}{ }^{\prime}=40 \mathrm{MPa}$ are limited to the cover. Under such high compressive stresses, these elements may be prone to crushing. For both loading cases, the columns with $200 \mathrm{~mm}$ and $300 \mathrm{~mm}$ spacing show an area of high stress extending diagonally from mid-height on the incident face throughout the core towards the distal face near the top support. As shown in Table 14, columns with transverse reinforcement spacing greater than $150 \mathrm{~mm}$ experience minimal increase in concrete strength due to confinement. Therefore, the areas of high effective stress extending throughout the core in these columns, as shown in Table 13, potentially indicates damage of core concrete. The absence of this high stress throughout the core in columns with spacing less than $150 \mathrm{~mm}$ highlights the effect of confinement. Providing sufficient amount of transverse reinforcement can prevent damage to the core concrete. 
Table 13: Effective stress distribution in columns at peak response for varying amount of transverse reinforcement spacing.

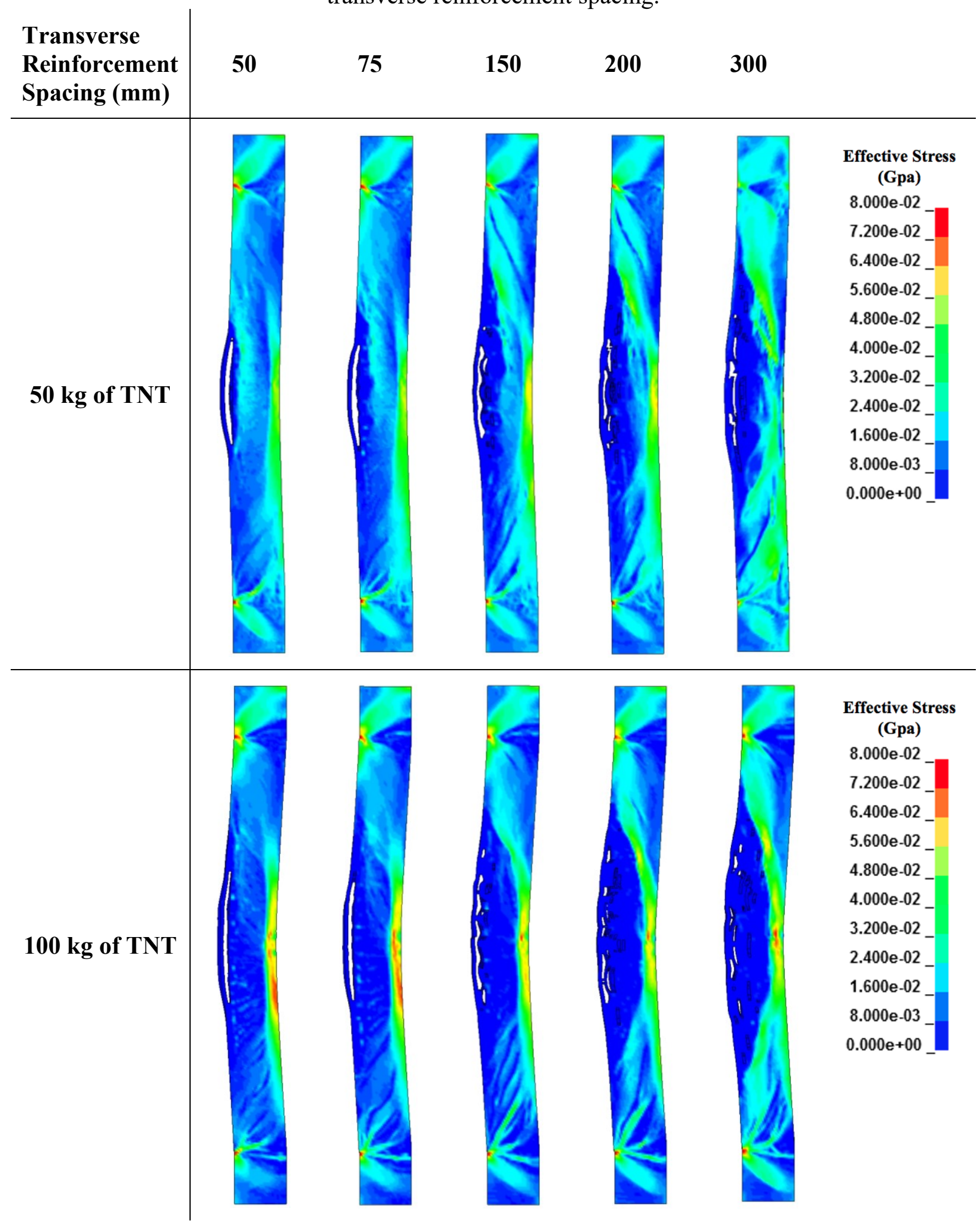




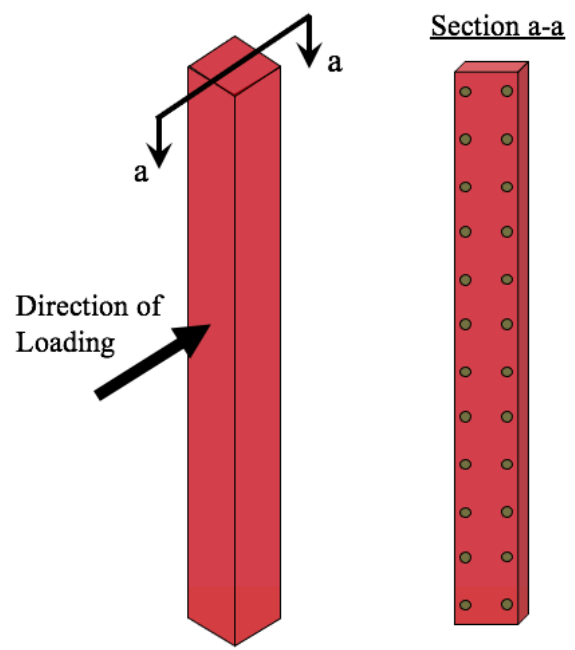

Figure 87: Section cut of columns for effective stress distributions.

Table 14: Effect of confinement on compressive strength, compressive strain, and axial capacity of columns with varying transverse reinforcement spacing.

\begin{tabular}{c|c|c|c}
$\begin{array}{c}\text { Transverse } \\
\text { Reinforcement } \\
\text { Spacing (mm) }\end{array}$ & $\begin{array}{c}\text { Compressive } \\
\text { strength of confined } \\
\text { concrete, f'cc (MPa) }\end{array}$ & $\begin{array}{c}\text { Compressive strain } \\
\text { of confined concrete } \\
\text { at peak strength, \&cc }\end{array}$ & $\begin{array}{c}\text { Maximum axial } \\
\text { capacity of } \\
\text { undamaged column, } \\
\mathbf{P}_{\mathbf{i}} \mathbf{( k N )}\end{array}$ \\
\hline 50 & 62.3 & 0.00757 & 4949 \\
75 & 54.1 & 0.00553 & 4673 \\
150 & 45.0 & 0.00326 & 4402 \\
200 & 42.7 & 0.00269 & 4352 \\
300 & 40.7 & 0.00218 & 4323
\end{tabular}

In blast resistant design, it is important that structural members be able to resist direct damage due to blast loads (i.e., damage sustained during the blast loading event). However, it is also critical for structural members to maintain their integrity after the blast in order to prevent progressive collapse. Therefore, once the blast-damaged columns had come to rest, they were subject to an increasing axial load until failure to assess their residual axial capacity.

The residual axial capacity of the columns was determined using load-displacement curves. Figure 88 shows the ratio of the residual axial capacity of the blast-damaged column to the maximum axial capacity of the undamaged column $\left(\frac{P_{r}}{P_{i}}\right)$. The maximum axial capacities of the undamaged columns with strength increase due to confinement effects were presented in Table 14. In Figure 
88 , it is evident that the transverse reinforcement spacing has a significant influence on the residual axial capacity. The results for residual axial capacity follow a linear trend similar to that observed for the length of spalling. Therefore, it can be concluded that local damage to the columns has a significant effect on the residual axial capacity. For both the $50 \mathrm{~kg}$ and $100 \mathrm{~kg}$ TNT loading cases, the residual axial capacity of the columns decreases with increasing transverse reinforcement spacing. For the $50 \mathrm{~kg}$ TNT loading case, all the columns were able to attain greater than $50 \%$ of their maximum axial capacity with the exception of the column with $300 \mathrm{~mm}$ spacing. In comparison, for the $100 \mathrm{~kg}$ TNT loading case, only the columns with transverse reinforcement spacing of $50 \mathrm{~mm}$ and $75 \mathrm{~mm}$ were able to attain greater than $50 \%$ of their maximum axial capacity. Again, the effects of confinement in these columns show a significant influence on their performance. The shear distress present in the columns with $150-300 \mathrm{~mm}$ spacing subject to 100 $\mathrm{kg}$ of TNT (refer to Table 12) likely also resulted in their decreased residual axial capacity. It can be concluded that transverse reinforcement has a significant effect on the local damage to the concrete and consequently produces a similar effect on the length of spalling and residual axial capacity of the column.

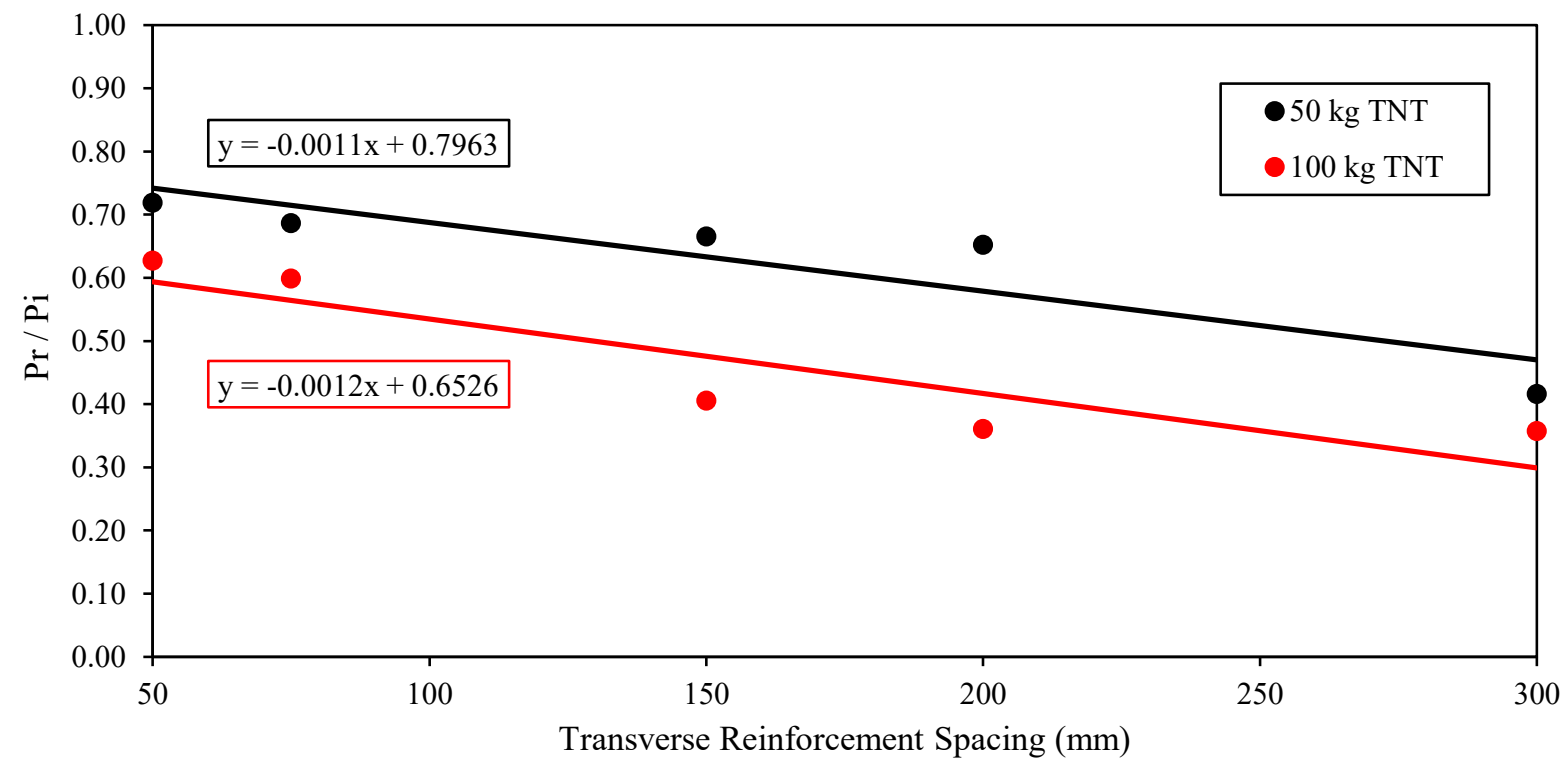

Figure 88: Effect of transverse reinforcement spacing on residual axial capacity. 


\subsubsection{Effect of Concrete Cover}

In order to analyze the effect of concrete cover, the cross-sectional dimensions of the column and the amount of steel reinforcement were kept constant. The longitudinal and transverse reinforcement was offset to achieve the desired amount of concrete cover, making the area of the core the only varying parameter. This was done to investigate the influence of the core to gross area $\left(\mathrm{A}_{\mathrm{c}} / \mathrm{A}_{\mathrm{g}}\right)$ ratio on column performance. The amount of concrete cover was measured from the outermost concrete cross-section layer to the outside edge of the transverse reinforcement. Concrete cover values from 0 to $60 \mathrm{~mm}$ were analyzed.

Figure 89 shows the effect of concrete cover on both maximum and residual lateral displacements. For the $50 \mathrm{~kg}$ TNT loading case, maximum displacement of the columns shows minor reductions when the concrete cover is reduced. The reduction in displacement is likely a result of the increasing area of core concrete as concrete cover decreases. Also, increasing the lever arm of internal forces when the concrete cover is reduced results in higher flexural strength. However, this improvement is almost negligible. The effect of concrete cover on residual displacements is also relatively minor. The residual displacements were roughly the same for columns with $0-40$ $\mathrm{mm}$ concrete cover. However, the residual displacement begins to increase when the concrete cover is increased past $50 \mathrm{~mm}$. This observation is also consistent with the effective strain distributions of the columns as presented in Table 15. Columns with concrete cover between $0-$ $40 \mathrm{~mm}$ show similar strain distributions. The columns all have areas of high strain at mid-height representing flexural cracking. However, a diagonal line of high strain extending from the distal face near the base support to the incident face at mid-height is visible in the column with $40 \mathrm{~mm}$ concrete cover. This area of high strain representing diagonal shear cracking increases in severity for the columns with $50 \mathrm{~mm}$ and $60 \mathrm{~mm}$ concrete cover. Furthermore, the region of high strain at 
mid-height of the columns with $50 \mathrm{~mm}$ and $60 \mathrm{~mm}$ concrete cover increases significantly. This observation is consistent with the trend in lateral displacements. The axial strain distribution in the longitudinal steel, as presented in Table 16, was relatively the same in all the columns subjected to $50 \mathrm{~kg}$ of TNT. However, the length of yielded longitudinal steel on the distal face of the columns slightly decreases with increasing concrete cover. Similarly, the magnitude of the strains decreases with increasing concrete cover.

For the $100 \mathrm{~kg}$ TNT loading cases, a similar trend is observed in the lateral displacements. The effect of concrete cover is minimal on maximum displacements. The residual displacements for columns with concrete cover between $0-40 \mathrm{~mm}$ remain relatively constant. However, no residual displacements were recorded for columns with $50 \mathrm{~mm}$ and $60 \mathrm{~mm}$ concrete cover as they failed in shear at the base support. These columns reached a maximum displacement and subsequently failed during the rebound portion of the response. The shear failure of these columns is visible in Table 15. The steel reinforcement also ruptured near the base support for both columns during the rebound portion of the response. Even at peak response a significant increase in strain is already visible near the base support (see Table 16). While reduction in concrete cover can result in a slight increase in confined strength of concrete, it does so at the expense of the area of the effectively confined core. The amount of confined core is a more important parameter when considering concrete cover. 


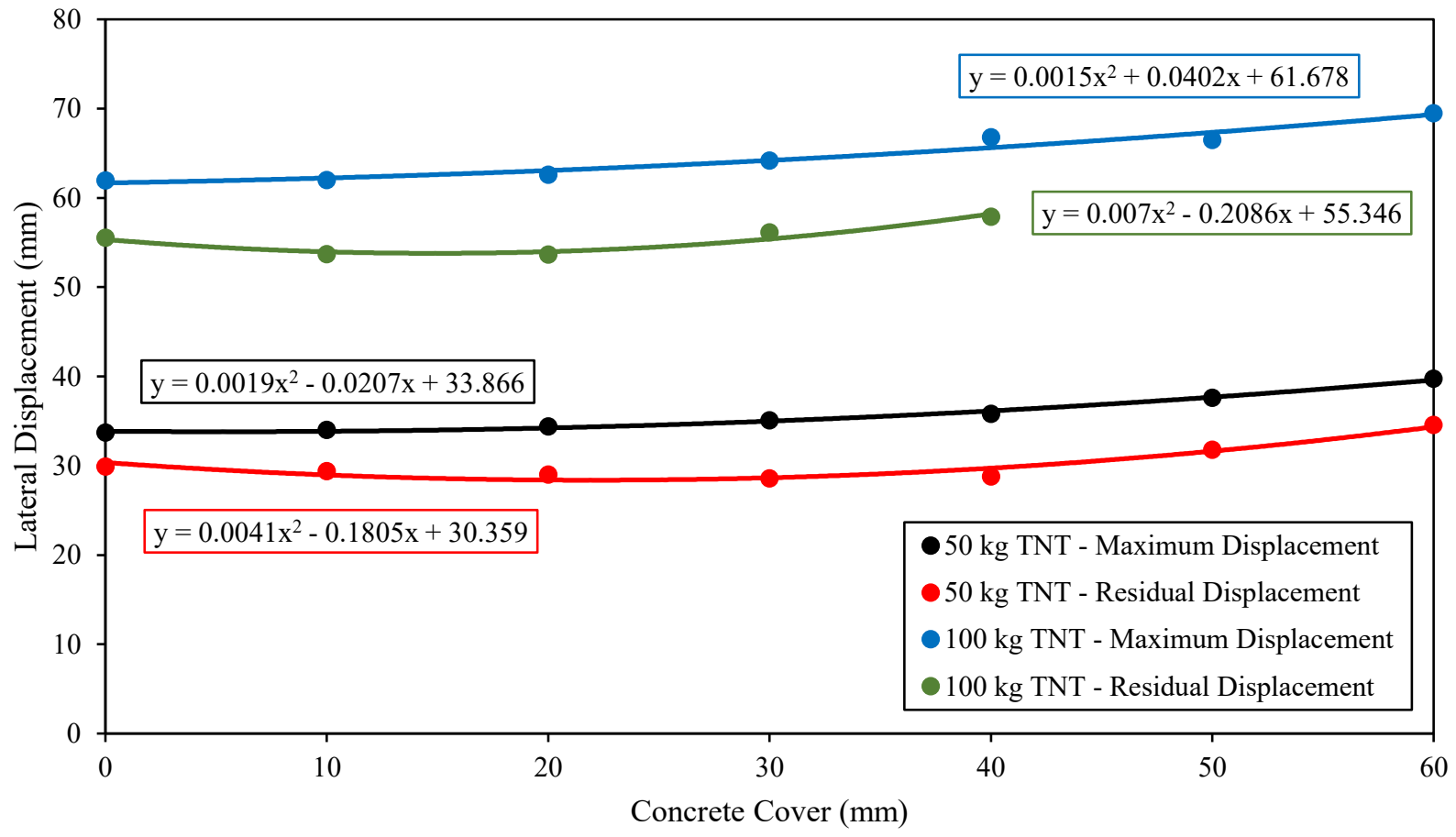

Figure 89: Effect of concrete cover on maximum and residual displacements. 
Table 15: Residual effective strain distribution in blast-damaged columns for varying amount of concrete cover.

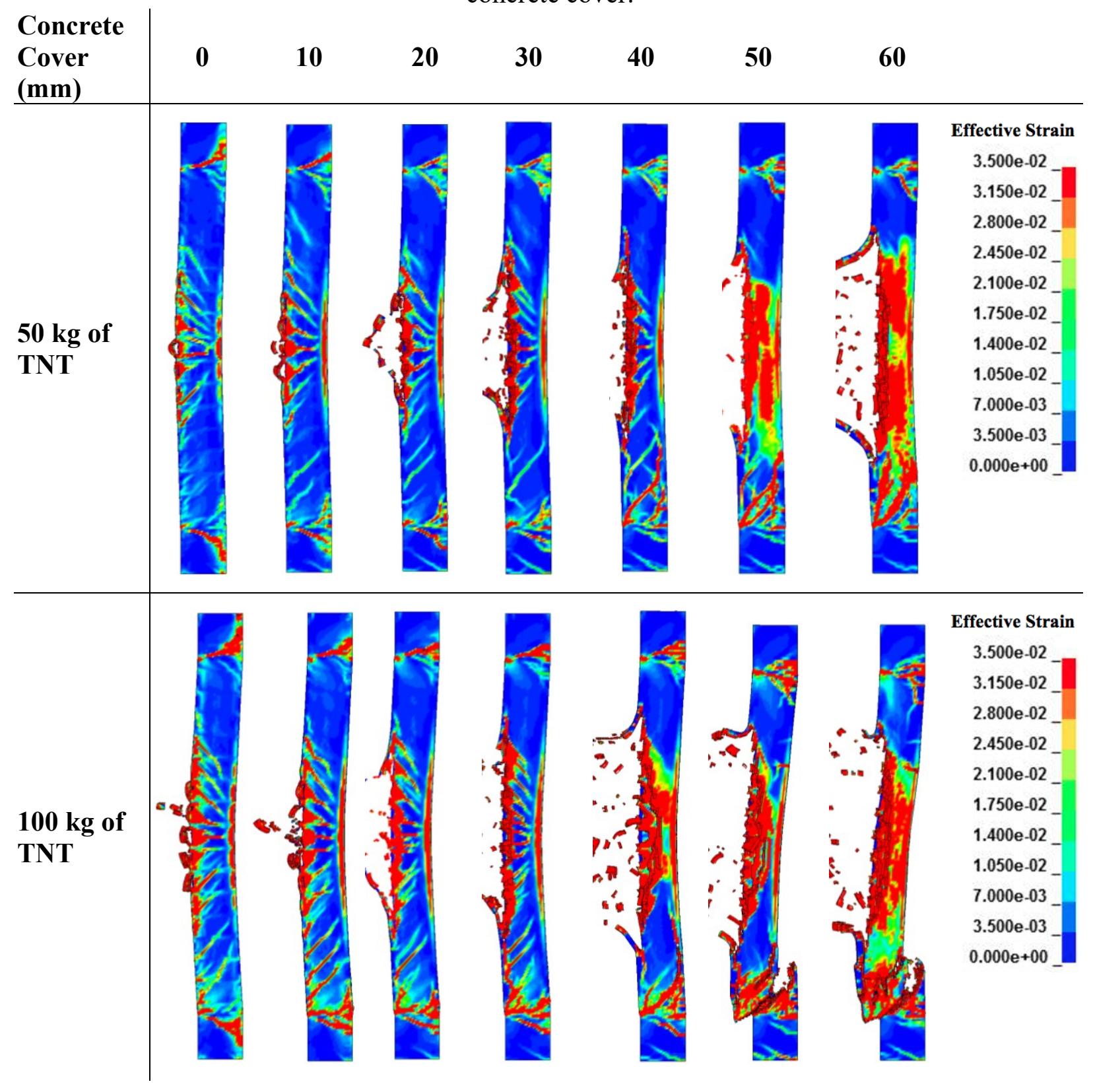


Table 16: Axial strain distribution in steel reinforcement at peak response for varying amount of concrete cover.

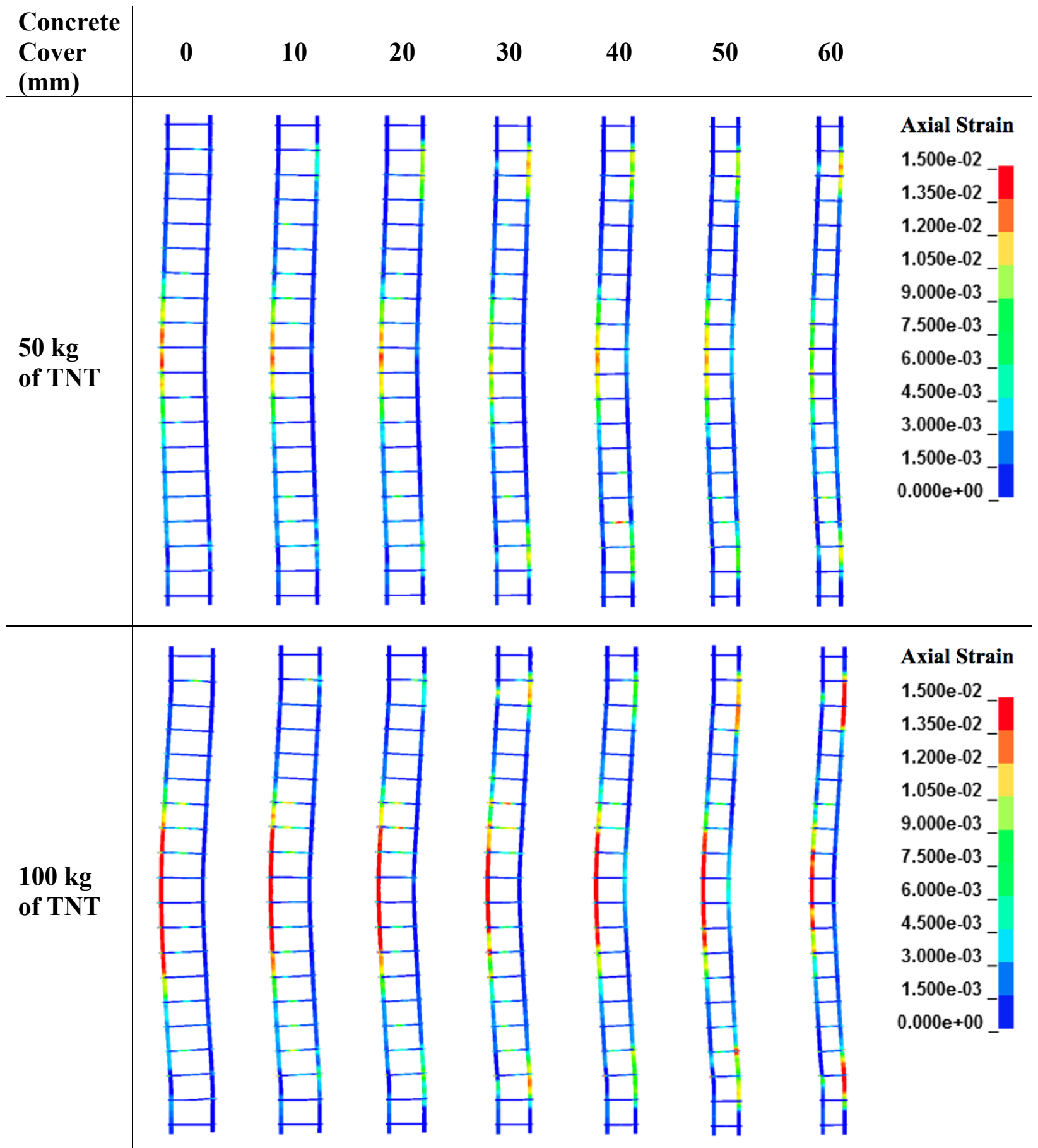

Figure 90 displays the effect of concrete cover on the length of spalling. The amount of spalling was significantly affected by the amount of cover. In most cases, the majority of the spalling tended to occur in the unconfined concrete cover. Therefore, it is reasonable that the reduction of concrete 
cover also resulted in a reduction in the degree of spalling. For both the $50 \mathrm{~kg}$ and $100 \mathrm{~kg} \mathrm{TNT}$ loading cases, as the concrete cover increased, so did the length of spalling. However, this trend was more pronounced in the columns subjected to $50 \mathrm{~kg}$ of TNT.

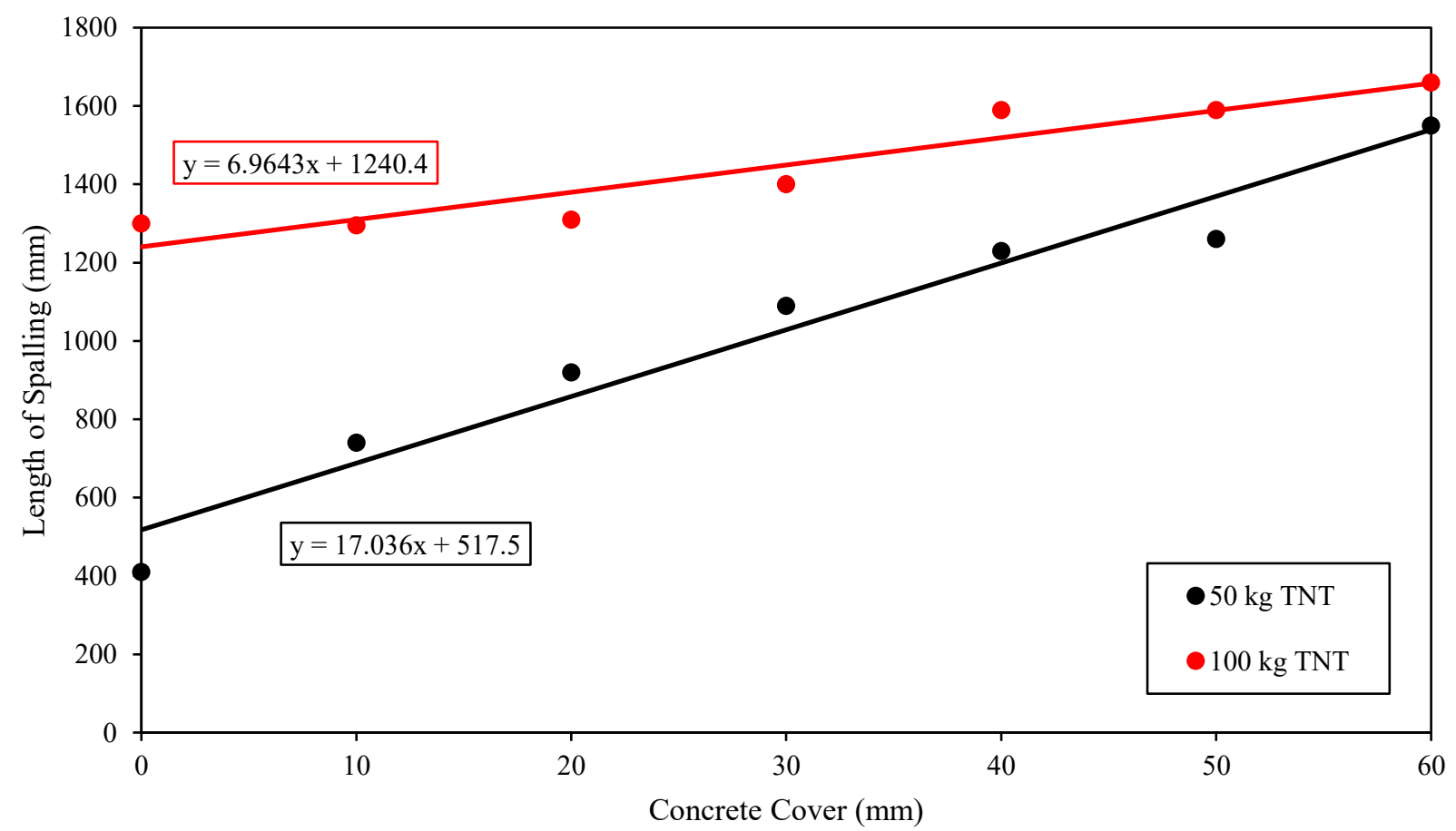

Figure 90: Effect of concrete cover on length of spalling.

The effective stress distribution of the columns at peak-response is presented in Table 17. The columns in this table show a section cut midway through the width of the columns, as previously detailed in Figure 87, so that the stresses within the core can be observed. Under both the loading conditions, the DIF for the concrete was computed to be 2.0 , resulting in a dynamic compressive strength of $80 \mathrm{MPa}$. The majority of the columns showed an area of high stress that was localized to the mid-height incident face of the columns within the concrete cover. These regions of high stress extended throughout the face of the column and indicate areas prone to possible crushing. Beginning with the column with $30 \mathrm{~mm}$ concrete cover, a small area of high stress is visible in the core just above mid-height. This area of high stress increases as the amount of concrete cover also 
increases, potentially indicating damage to concrete in the core. This again supports the conclusion that the columns with lower amounts of concrete cover provide better confinement to the core.

Table 17: Effective stress distribution in columns at peak response for varying amount of concrete cover.

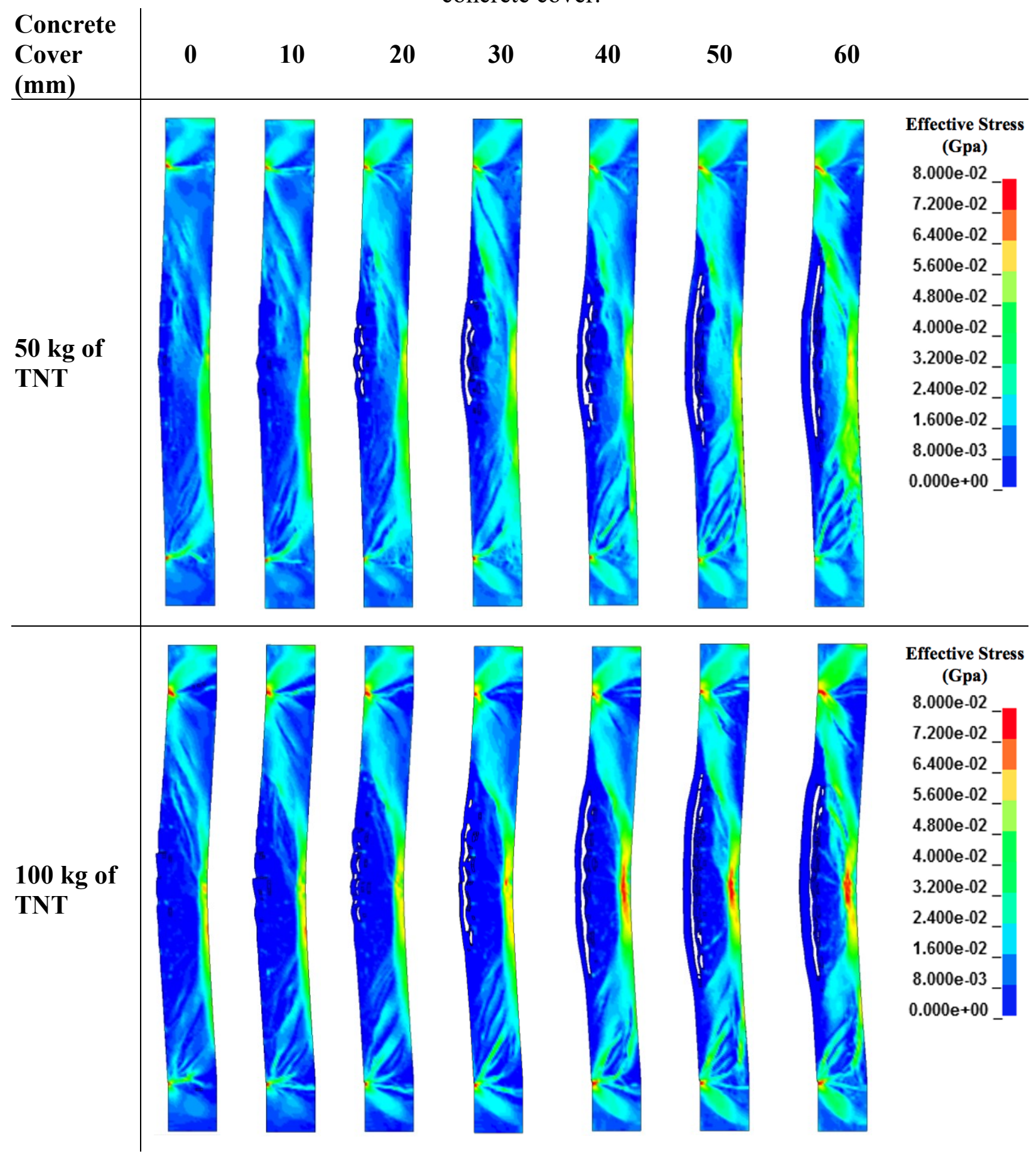


The effect of concrete cover on the ratio of the residual axial capacity of the blast-damaged column to the maximum axial capacity of the undamaged column $\left(\frac{P_{r}}{P_{i}}\right)$ is shown in Figure 91. The maximum axial capacities of the undamaged columns with strength increase due to confinement effects are presented in Table 18. The effect of concrete cover on the confined strength of concrete is minimal, but its effect on residual axial capacity is quite significant. This is due to the change in effectively confined core area. For both the $50 \mathrm{~kg}$ and $100 \mathrm{~kg}$ loading cases, columns with concrete cover of $0-40 \mathrm{~mm}$ tend to result in similar residual axial capacities. However, for columns with concrete cover greater than $40 \mathrm{~mm}$, the residual axial capacity begins to decrease. This eventually leads to no residual axial capacity of the columns with $50 \mathrm{~mm}$ and $60 \mathrm{~mm}$ cover subjected to 100 $\mathrm{kg}$ of TNT as they failed during the blast loading. This trend is similar to what was observed in the lateral displacements as well as the effective strain distribution in the columns.

Cui et al. (2015) evaluated the performance of two columns with different concrete cover thicknesses subjected to blast loading. It was found that the smaller concrete cover resulted in a lower damage level, based on the ratio of residual load carrying capacity post-blast to that of an undamaged column. The present study found that concrete cover values between 0 and $40 \mathrm{~mm}$ resulted in similar lateral displacements and residual axial capacities for the investigated crosssection. However, an increase in concrete cover past $40 \mathrm{~mm}$ reduced column performance. 
Table 18: Effect of confinement on compressive strength, compressive strain, and axial capacity

\begin{tabular}{c|c|c|c}
$\begin{array}{c}\text { Concrete Cover } \\
(\mathbf{m m})\end{array}$ & $\begin{array}{c}\text { of columns with varying concrete cover. } \\
\text { Compressive } \\
\text { strength of confined } \\
\text { concrete, f'cc (MPa) }\end{array}$ & $\begin{array}{c}\text { Compressive strain } \\
\text { of confined concrete } \\
\text { at peak strength, \&cc }\end{array}$ & $\begin{array}{c}\text { Maximum axial } \\
\text { capacity of } \\
\text { undamaged column, } \\
\mathbf{P}_{\mathbf{i}}(\mathbf{k N})\end{array}$ \\
\hline 0 & 44.3 & 0.00307 & 4441 \\
10 & 44.5 & 0.00313 & 4429 \\
20 & 44.8 & 0.00319 & 4416 \\
30 & 45.0 & 0.00326 & 4402 \\
40 & 45.3 & 0.00332 & 4389 \\
50 & 45.5 & 0.00338 & 4375 \\
60 & 45.7 & 0.00342 & 4361
\end{tabular}

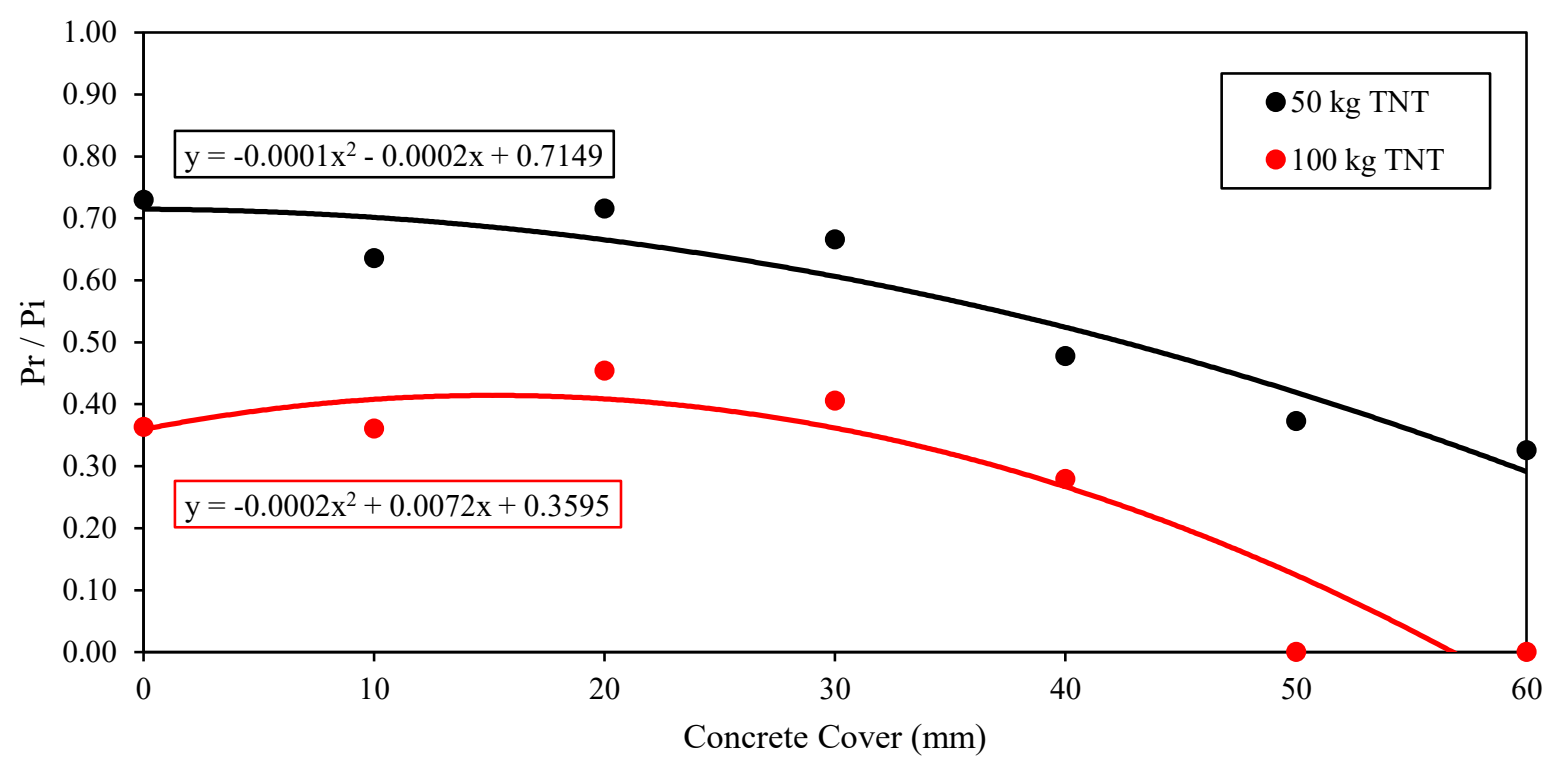

Figure 91: Effect of concrete cover on residual axial capacity. 


\subsubsection{Effect of Axial Loading}

To examine the effect of axial load ratio, five different axial load levels under the $50 \mathrm{~kg}$ and 100 $\mathrm{kg}$ TNT blast load cases were evaluated. Column details were that of the reference column provided in Section 6.1. Axial load ratios ranging from $0 \%$ to $60 \%$ were evaluated. Figure 92 shows the effect of axial loading on maximum and residual lateral displacements. It is evident that increasing axial loading generally results in lower maximum and residual displacements. Increasing the axial loading on the columns reduced displacements by increasing the confinement of the column in the longitudinal direction. Axial loading also improves the effective stiffness of concrete by providing initial compression in the column, similar to the effect caused by prestressing.

However, this trend reaches a critical point, after which there is no further improvement in the resistance. For the $50 \mathrm{~kg}$ TNT loading case, the numerical simulation for an axial load ratio of 0.6 resulted in shear failure of the column, which is why no meaningful residual displacement data is recorded. The column reached a maximum displacement but was unable to recover during rebound and instead experienced a rapid degradation of strength due to buckling of reinforcement near the fixed base of the column. A similar response was observed for the $100 \mathrm{~kg}$ TNT loading case, for axial load ratios of 0.45 and 0.6 . The final deflected shapes of the columns with effective strain distribution are shown in Table 19, where the shear failure can be observed at the base of these columns.

In Table 19, it is evident that as the axial load ratio increases, the degree of shear distress near the base support also increases. The column with no axial loading applied was torn from the base support during the blast loading for both the $50 \mathrm{~kg}$ and $100 \mathrm{~kg}$ TNT loading cases. This is due to 
the fact that the top support does not restrain movement in the vertical direction. As previously mentioned, this was done to allow the transmission of axial loads.

In the $50 \mathrm{~kg}$ TNT loading case, the columns with axial load ratios of 0.15 and 0.3 show a similar pattern of strain at the base support. However, the column with an axial load ratio of 0.45 shows an increase in strain near the support. The diagonal line of high strain extending from the base support on the distal face to just below mid-height on the incident face represents a large shear crack. This diagonal damage zone increases for the column with an axial load ratio of 0.6 and ultimately governs its failure. Based on this, it would appear that increasing the axial load ratio past approximately 0.3 has a negative effect on the behaviour of the column which may result in brittle shear failures. Also, for columns with extremely low levels of axial load, longitudinal movement needs to be restrained to prevent axial tensile failure at the support.

For the $100 \mathrm{~kg}$ TNT loading case, the column with no axial loading again experiences tensile failure at the base of the support due to the absence of restraint in the longitudinal direction. This column also experiences a significantly higher amount of damage throughout its height. The columns with axial load ratios of 0.45 and 0.6 fail in shear at the base support.

The axial strain distribution in the steel reinforcement at peak response is presented in Table 20. Under both the loading conditions, the DIF for the steel reinforcement was calculated to be approximately 1.4, resulting in a dynamic yield strength and strain of $560 \mathrm{MPa}$ and 0.0028 , respectively. The magnitude of the strains at mid-height of the distal face decrease with increasing axial load ratio. The length of yielded longitudinal steel elements also decreases. This is consistent with the lateral displacement results (see Figure 92).

In comparison, Thai and Kim (2018) found that as axial loading was increased, damage to slender columns also increased. However, the study found that axial loading had a minimal effect on 
stocky columns, aside from a slight decrease in maximum deflection. The results of the present parametric study complement these results as they evaluate a special case of failure in RC columns under near-field blast. The results of this study show that as axial loading increases, the failure mode of the column shifts from flexure to shear, as indicated by the formation of the shear crack as the base of the column. Shear failure is a brittle failure type and inherently results in lower maximum and residual displacements. In contrast, Thai and Kim (2018) evaluated two separate failure cases, slender columns responding in flexural failure and stocky columns responding in shear failure.

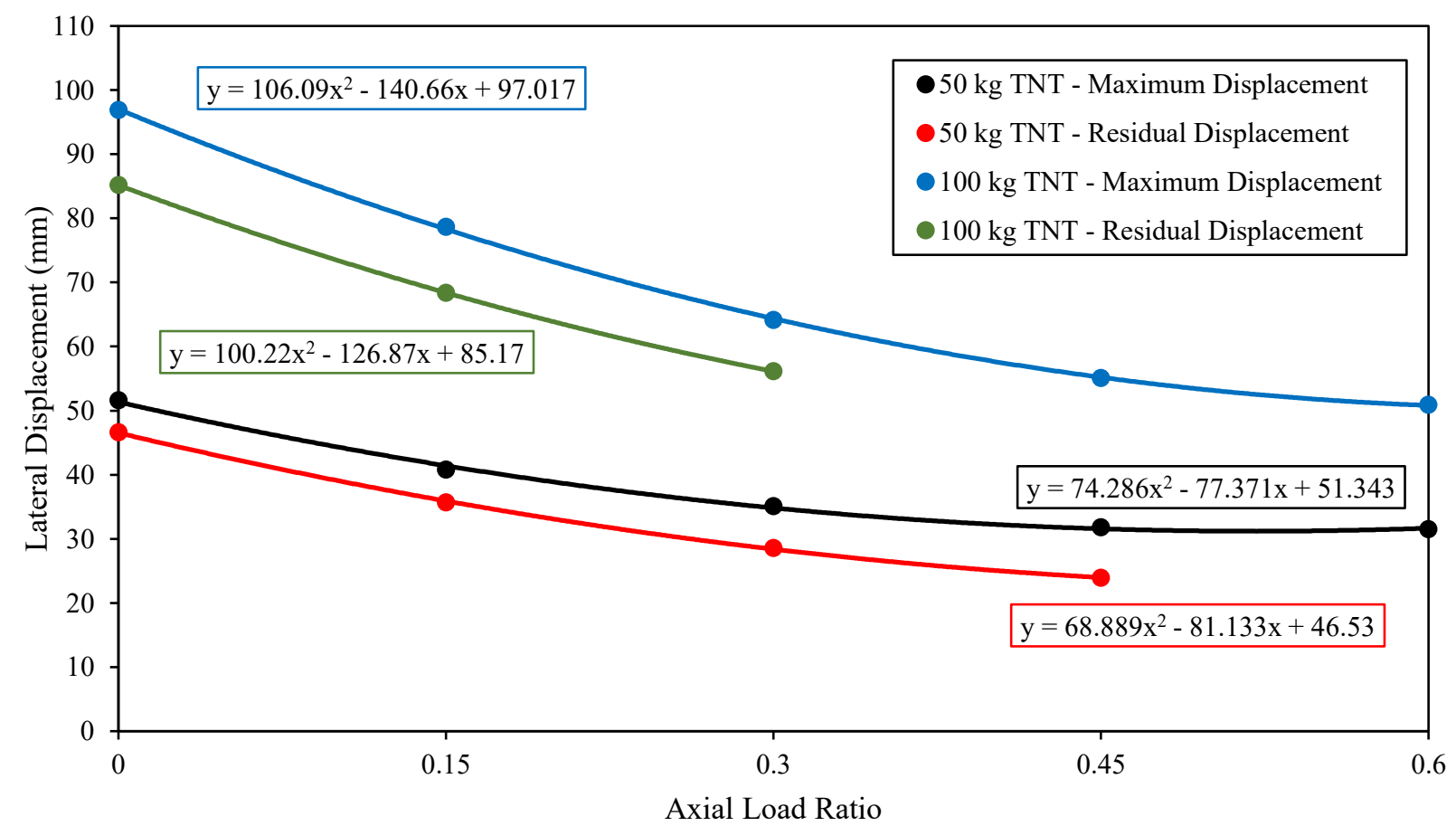

Figure 92: Effect of axial load ratio on maximum and residual displacements. 
Table 19: Residual effective strain distribution in blast-damaged columns for varying axial load

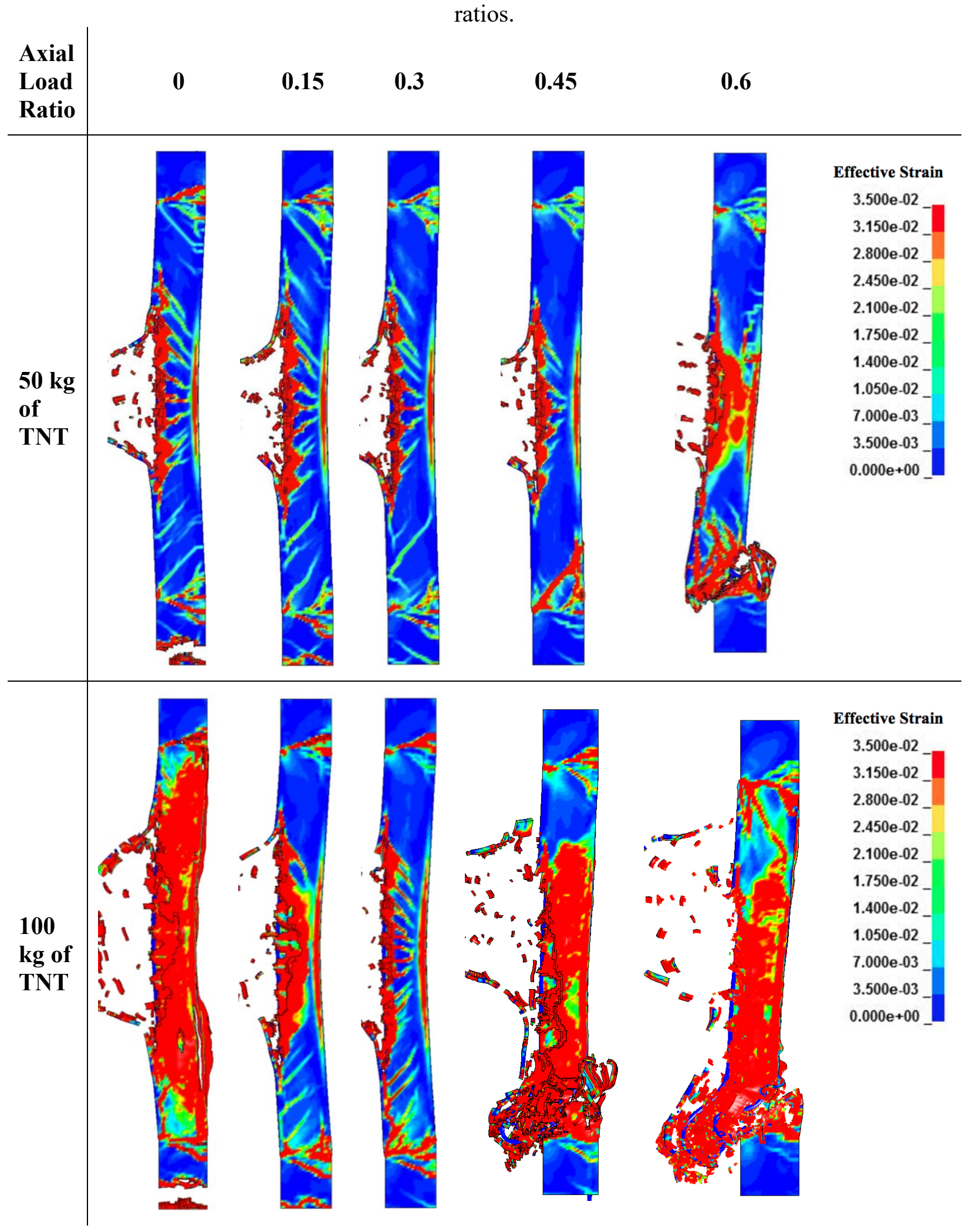


Table 20: Axial strain distribution in steel reinforcement at peak response for varying axial load ratios.

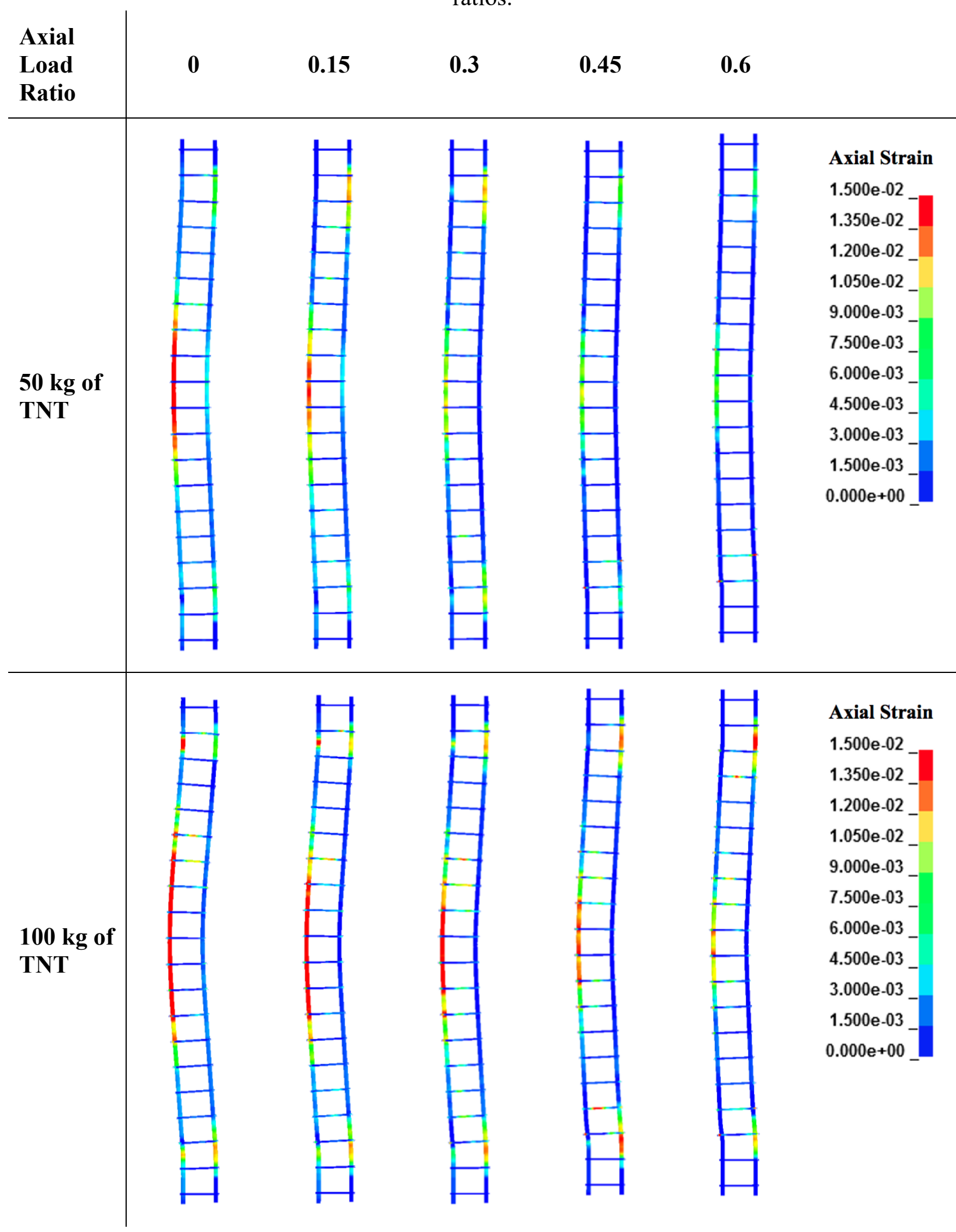


The effect of axial load ratio on the length of spalling is shown in Figure 93, which shows that the length of spalling tends to decrease with increasing axial load ratio. This is likely due to the increase in the confinement of the column in the longitudinal direction as a result of the axial loading. The columns with higher axial load ratios also resulted in lower lateral displacements. As a result, the degree of tensile stress on the distal face of the column reduces, consequently reducing length of spalling. Furthermore, the reduction in spall length may be a result of the changing damage mechanism from flexure to shear. The spalling measured at mid-span is directly related to flexural damage. Therefore, as the failure mode of the column transitions to shear failure, the spall length decreases.

Figure 94 presented the effect of axial load ratio on the ratio of the residual axial capacity of the blast-damaged column to the maximum axial capacity of the undamaged column $\left(\frac{P_{r}}{P_{i}}\right)$. The case of no axial loading resulted in tearing close the base support during the blast loading phase, so no meaningful data is recorded for residual axial capacity. The residual axial capacities of the columns with an axial load ratio of 0.15 and 0.3 are very similar for both the $50 \mathrm{~kg}$ and $100 \mathrm{~kg}$ TNT loading cases. For both loading scenarios, the column with an axial load ratio of 0.6 had no residual axial capacity as it failed in shear during the blast loading. This also occurred for the column with an axial load ratio of 0.45 when subjected to $100 \mathrm{~kg}$ of TNT.

The effective stress distributions of the columns for both loading scenarios are presented in Table 21. The columns in this table show a section cut midway through the width of the columns, as previously detailed in Figure 87, so that the stresses within the core can be observed. Under both the loading conditions, the DIF for the concrete was computed to be 2.0 , resulting in a dynamic compressive strength of $80 \mathrm{MPa}$. For the columns with an axial load ratio of 0.45 and 0.6 that were 
subjected to $100 \mathrm{~kg}$ of TNT, a diagonal line of high stress is visible extending from mid-height on the incident face to the top support on the distal face. This stress indicates possible damage to core concrete and likely also contributed to the failure of these columns during the rebound portion of the response. A similar line of high stress can be seen forming in these columns when subjected to $50 \mathrm{~kg}$ of TNT as well. Again, this likely led to the decrease in residual axial load capacity for these columns. For both loading scenarios, the areas under tension reduce as the axial load ratio increases. This is consistent with the reduced lateral displacements and length of spalling observed in the columns as axial load ratio is increased.

For both loading cases, axial load ratios of $0.15-0.3$ appear to provide adequate resistance to the blast loading so that maximum and residual displacements are significantly reduced while maintaining the post-blast integrity of the column. The effective strain distribution of these columns is also similar in both loading scenarios. However, increasing the axial load ratio past 0.3 seems to have a negative effect on the residual axial capacity of the column, eventually leading to shear failure of the column.

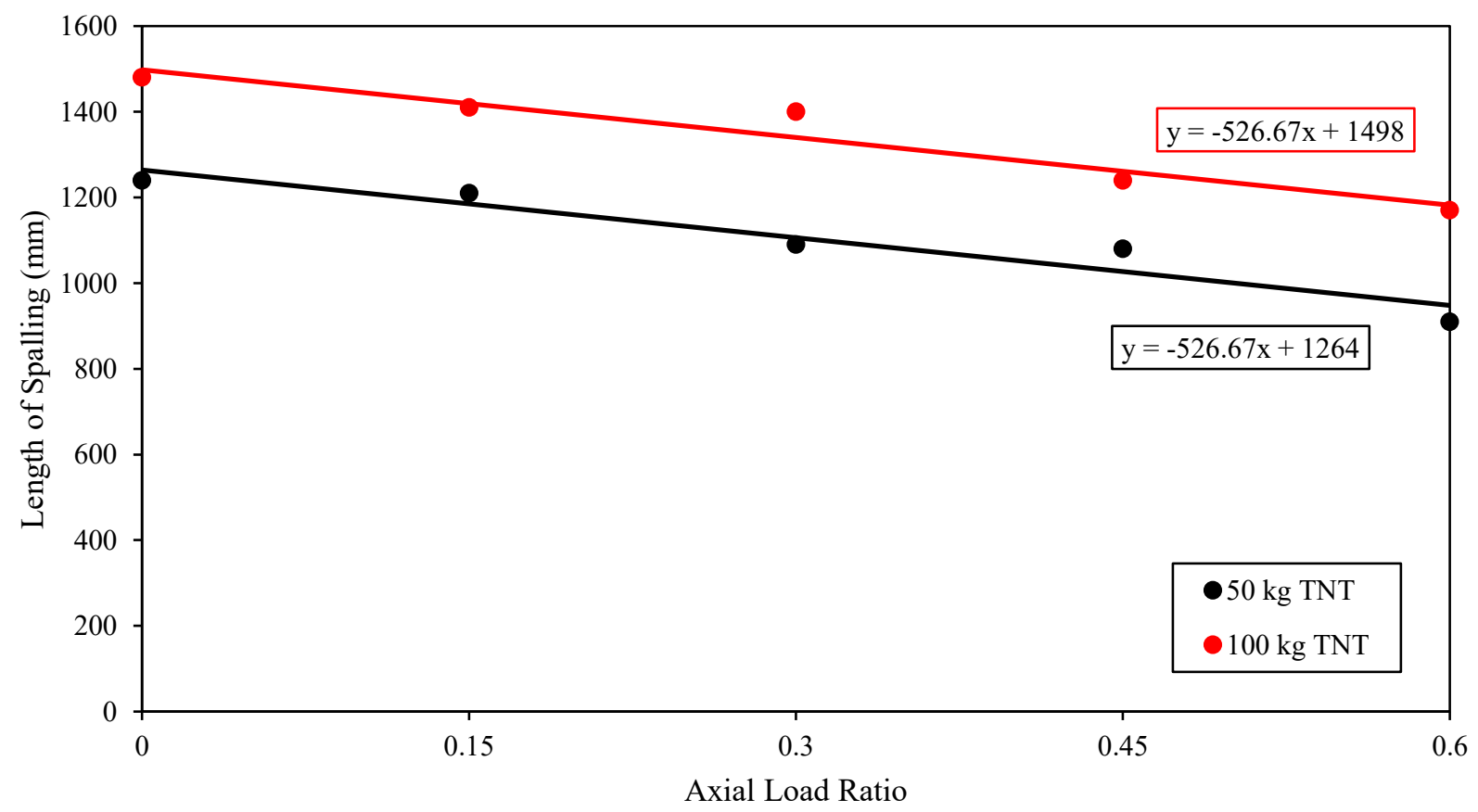


Figure 93: Effect of axial load ratio on length of spalling.

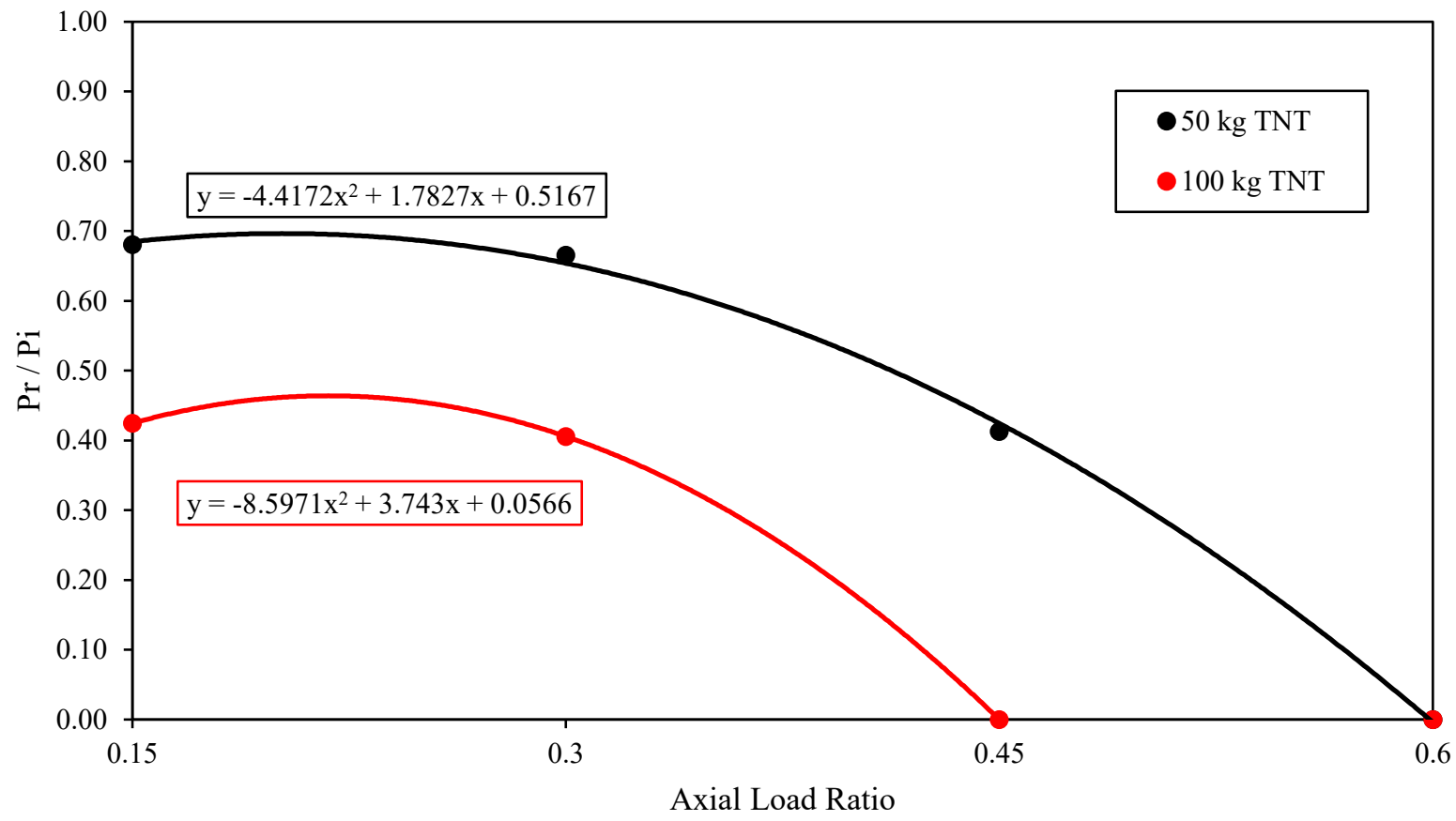

Figure 94: Effect of axial load ratio on residual axial capacity. 
Table 21: Effective stress distribution in columns at peak response for varying axial load ratios.

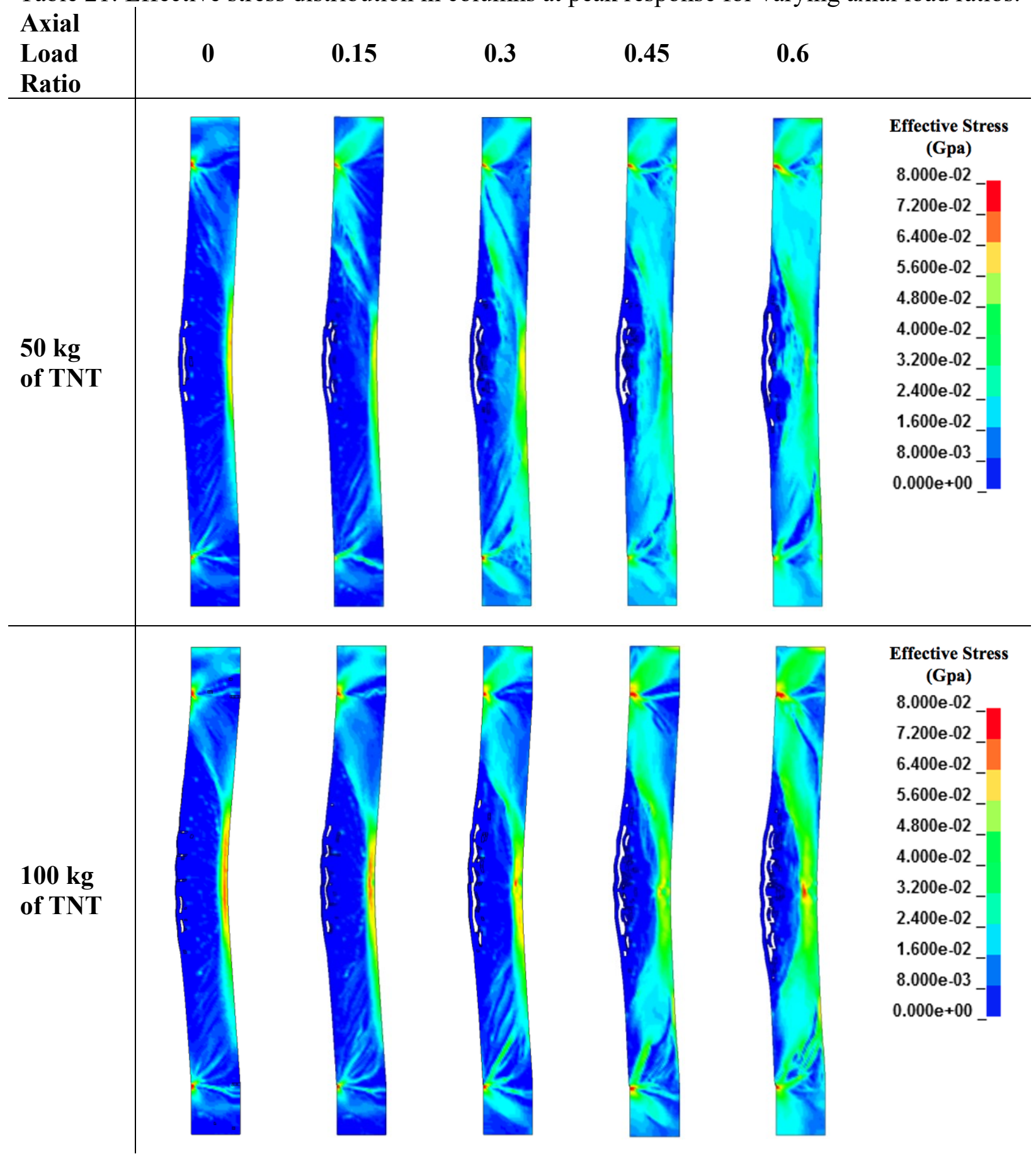

The axial load-moment interaction (P-M) interaction diagram of the reference column, and consequently the column used to evaluate axial load ratio, is presented below in Figure 95. Based on the previously discussed performance measures, it was observed that increasing axial load ratio 
past approximately 0.3 had a negative effect on column behaviour. As outlined in Section 6.1, the magnitude of load corresponding to an axial load ratio of 0.3 is $844 \mathrm{kN}$.

The observed trend wherein column performance reaches an optimal value is similar to the trend observed in the P-M interaction diagram. As axial loading increases, the moment capacity of the column also increases. This trend reaches a maximum, referred to as the balanced point, after which there is a reduction in moment capacity. In Figure 95 below, the balanced point for this column corresponds to an axial load value of $843 \mathrm{kN}$. The axial load ratio of 0.3 , after which column behaviour under blast is negatively affected by an increase in axial loading, corresponds with the balanced point in Figure 95. Based on this observation, it appears that a relationship may exist between the axial load associated with balanced failure of an RC column and the axial load associated with optimal performance of RC columns under blast. However, a more comprehensive analysis of such a relationship, one that extends to a variety of cross-sections and reinforcement conditions, would be required to draw a general conclusion.

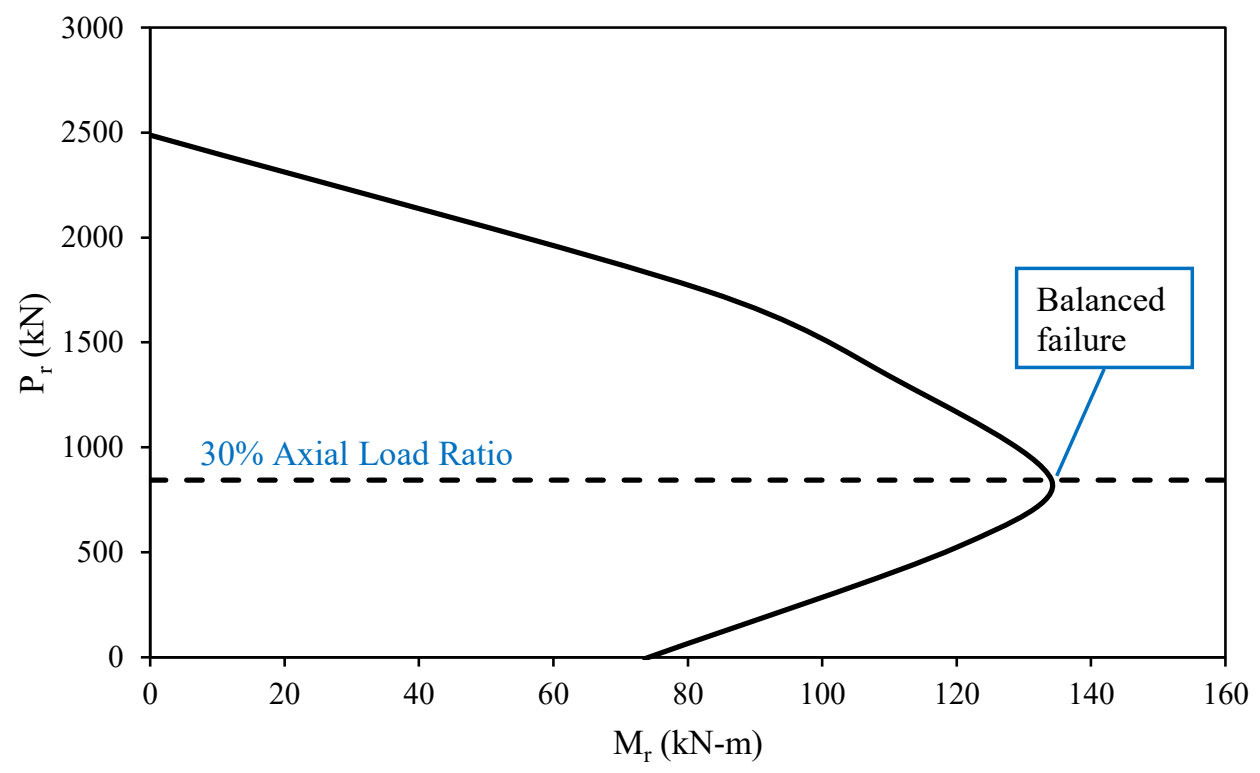

Figure 95: P-M interaction diagram for the reference column. 


\subsubsection{Effect of Column Shape}

To analyze the effect of column cross-section shape, the following cross-sections were tested: square, rectangular (with bending about the weak axis), rectangular (with bending about the strong axis), and circular. The reinforcement detailing and cross-section dimensions of the columns are shown in Figure 96. In order to maintain the same amount of steel reinforcement across all the cross-sections, the amount of longitudinal steel was changed to 6-20M bars. Transverse reinforcement and spacing were kept the same as the reference column. The direction of the blast loading is also indicated on the diagrams.

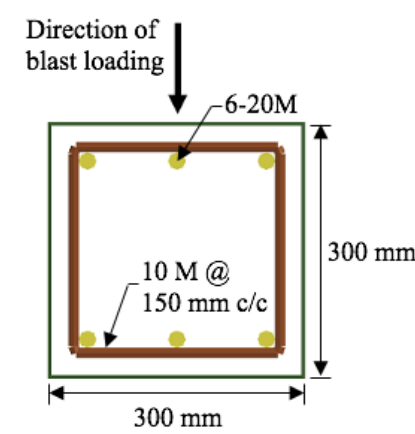

(a)

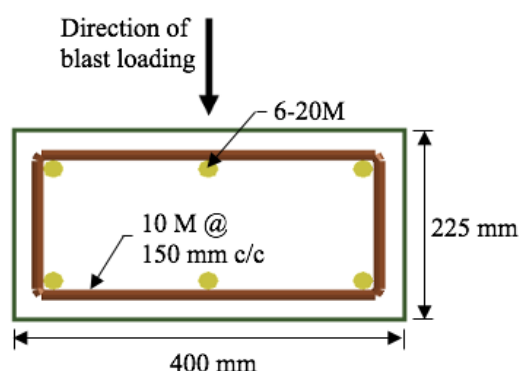

(b)

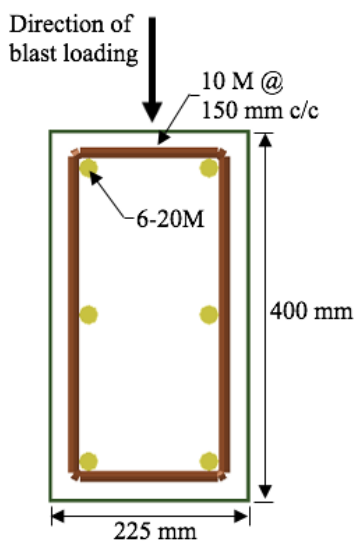

(c)

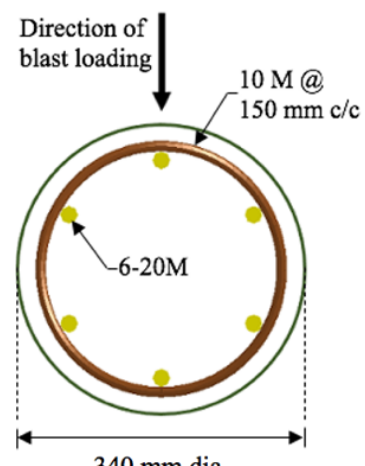

(d)

Figure 96: Column shapes and cross-section detailing. (a) Square (b) Rectangular (bending about weak axis) (c) Rectangular (bending about strong axis) (d) Circular

Figure 97 shows the effect of column cross-section shape on the maximum and residual lateral displacements, while Figure 98 shows the effect of shape on the length of spalling. In both graphs, it is evident that the circular column performs best under both blast loading scenarios. This is likely due to the fact that the magnitude of the reflected blast load is affected by structure geometry. Reflected pressures vary based on the angle of incidence. The angle of incidence is defined as the angle measured between the outward normal of the reflecting surface and a radial line from the charge to the point of incidence. An angle of incidence of $0^{\circ}$ results in a normally reflected blast 
wave while an angle of incidence of $90^{\circ}$ results in no reflection (and therefore no increase in pressure or impulse). Based on the geometry, the angle of incidence to a point on the front face of the circular column will be greater than that of the square or rectangular column, resulting in less reflection.

However, the column with bending about the strong axis had a smaller amount of surface area exposed to the reflected blast pressures than the other columns. This factor along with the increased moment resistance of this column resulted in lower displacements, which were comparable to that of the circular column. The rectangular column with bending about the weak axis has the greatest surface area exposed to the blast wave and therefore has a higher area subjected to reflected pressures, which results in significantly larger displacements. This column was not able to resist the second blast loading scenario of $100 \mathrm{~kg}$ of TNT, and therefore no displacement or spalling data was reported in Figure 97 and Figure 98.

In Figure 98, the square cross-section and the rectangular cross-section with bending about the weak axis had the most severe spalling. The rectangular cross-section with bending about the strong axis resulted in less spalling, indicating that the depth of the member has an effect on local damage. Similar to the displacement results, the column with the circular cross-section performed the best under the blast load, resulting in the least amount of spalling. This is likely due to the same reasons discussed earlier. Furthermore, the spiral reinforcement provided better confinement to the core and reduced the degree of spalling.

Rajkumar et al. (2020) found a similar observation when investigating column shape at scaled distances greater than $0.75 \mathrm{~m} / \mathrm{kg}^{1 / 3}$. The study concluded that circular columns performed better in terms of peak deflections, but it was also noted that some discrepancies in residual deflections existed. The present study also found that that the circular column showed optimal performance 
under blast loads at scaled distances less than $0.4 \mathrm{~m} / \mathrm{kg}^{1 / 3}$ for both maximum and residual deflections. The superior performance of the circular column is also supported in regard to local damage in terms of spall length.

Table 22 presents the effective strain distribution in the blast-damaged columns once they had come to rest. The square cross-sections show flexural cracking at the mid-height region of the columns, and the severity of the damage increases when the charge mass is increased to $100 \mathrm{~kg}$. In the $100 \mathrm{~kg}$ TNT loading case, the damage extends throughout the depth of the column for the square cross-section. This is likely the reason for the significantly higher deflection reported. The rectangular cross-section with bending about the strong axis shows an increase in the degree of damage on the distal face of the column. When subjected to $50 \mathrm{~kg}$ of TNT, this column shows a moderate degree of damage throughout the column depth. However, when subjected to $100 \mathrm{~kg}$ of TNT, the damage distribution changes and instead shows the formation of flexural cracks. The extent of the damage in the circular member tends to be localized to the mid-height region as opposed to the damage in the other columns where it extends throughout the height of the column. Table 23 presents the axial strain distribution in the steel reinforcement of the blast-damaged columns. Under both the loading conditions, the DIF for the steel reinforcement was calculated to be approximately 1.4, resulting in a dynamic yield strength and strain of $560 \mathrm{MPa}$ and 0.0028 , respectively. All the columns showed yielding in the longitudinal steel reinforcement at mid-height of the distal face and at the support on the incident face. None of the columns showed rupturing of reinforcement. The rectangular column with bending about the weak axis had the highest magnitude of strains. In contrast, the circular column had the lowest magnitude of strains, both of which are consistent with the lateral displacement results. 


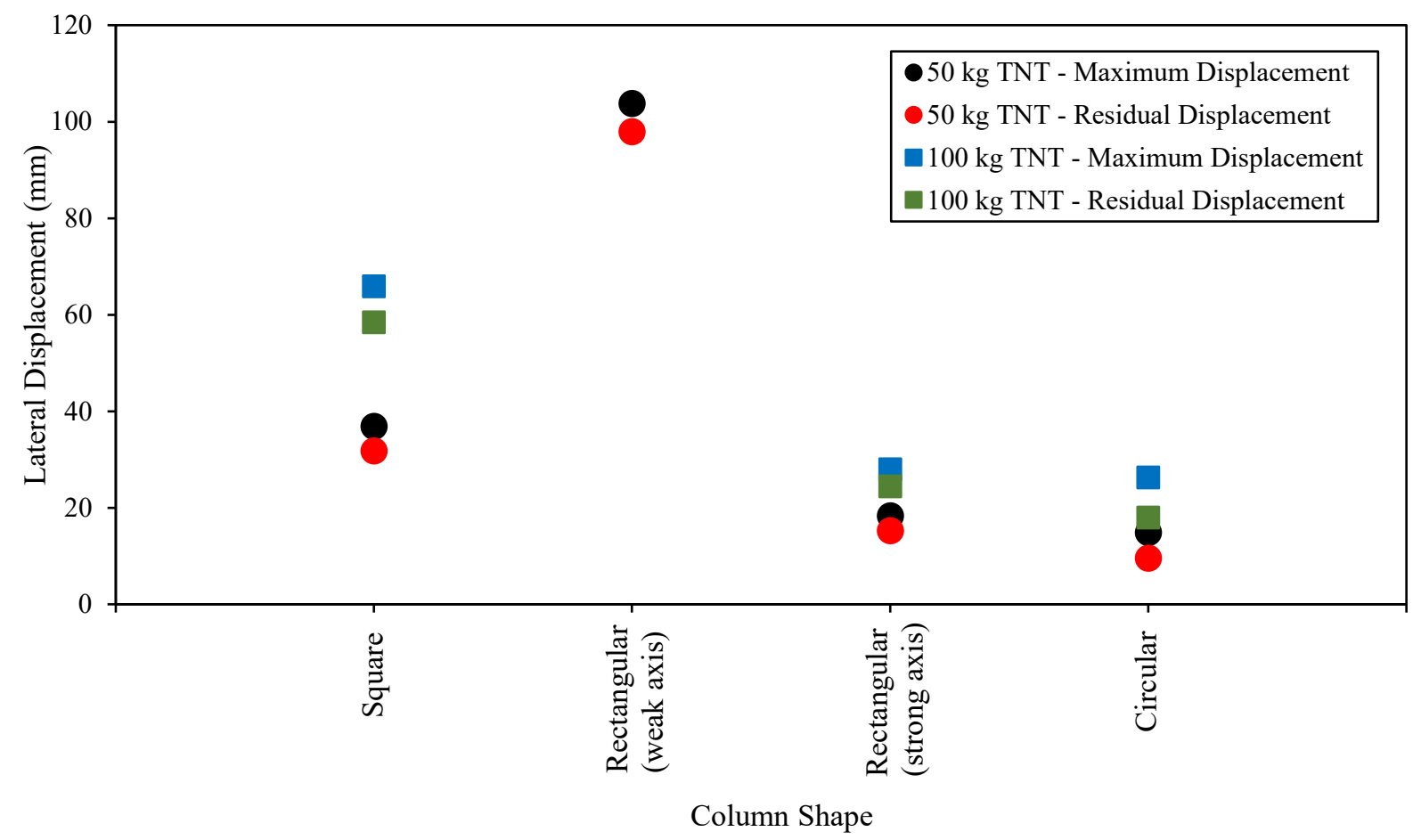

Figure 97: Effect of column shape on maximum and residual displacements.

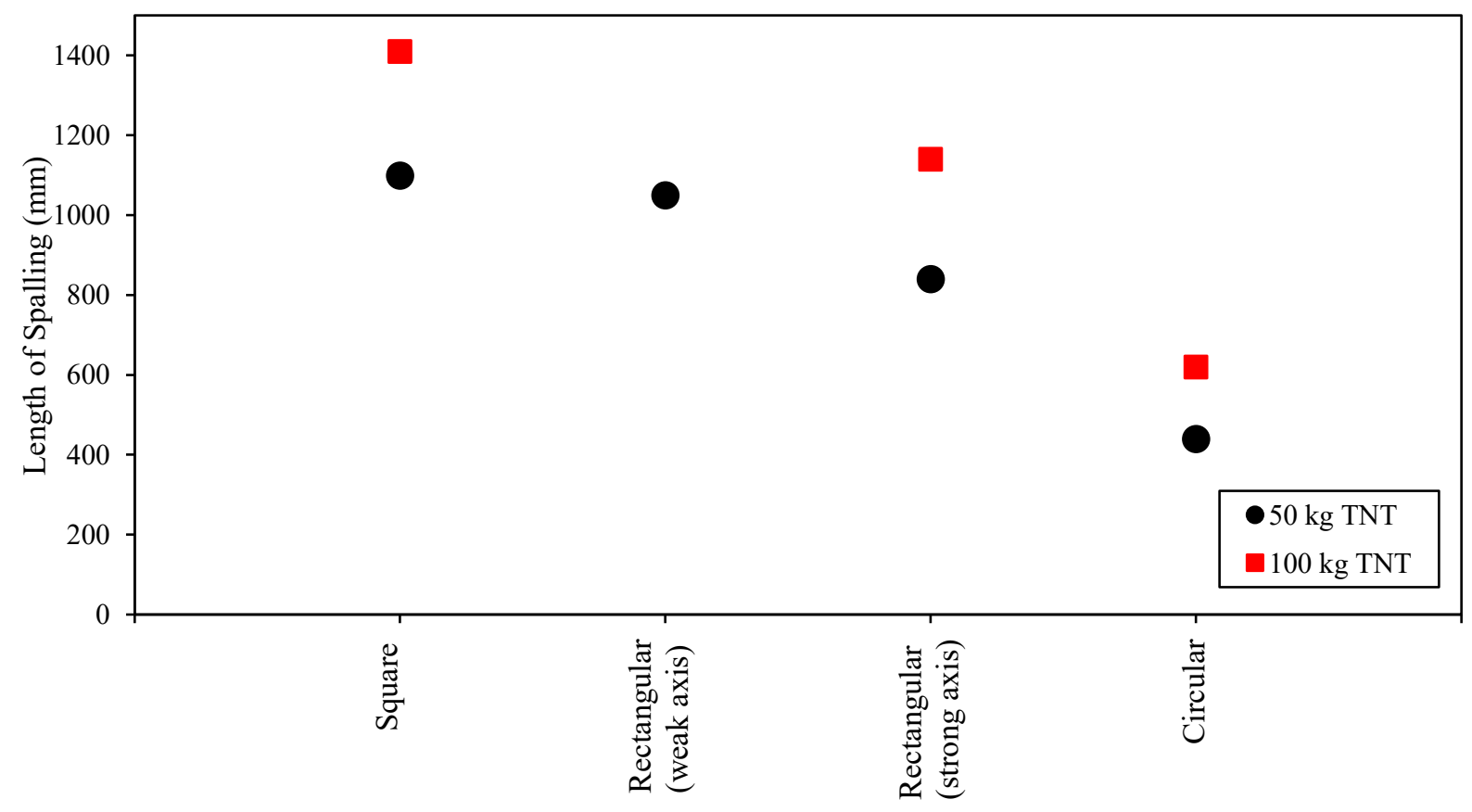

Column Shape

Figure 98: Effect of columns shape on length of spalling. 
Table 22: Effective strain distribution in blast-damaged columns for various cross-section shapes.

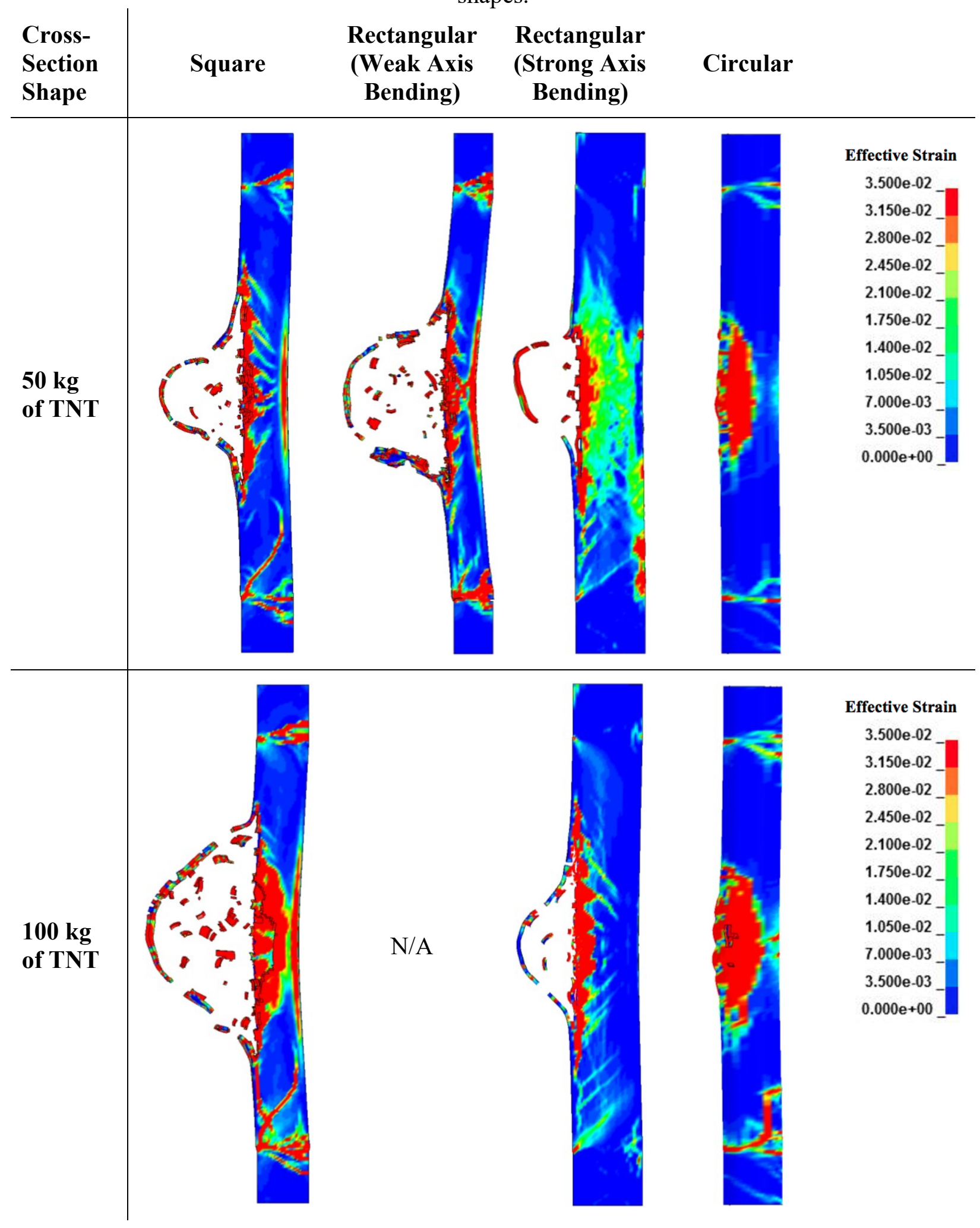


Table 23: Axial strain distribution in steel reinforcement at peak response for various crosssection shapes.

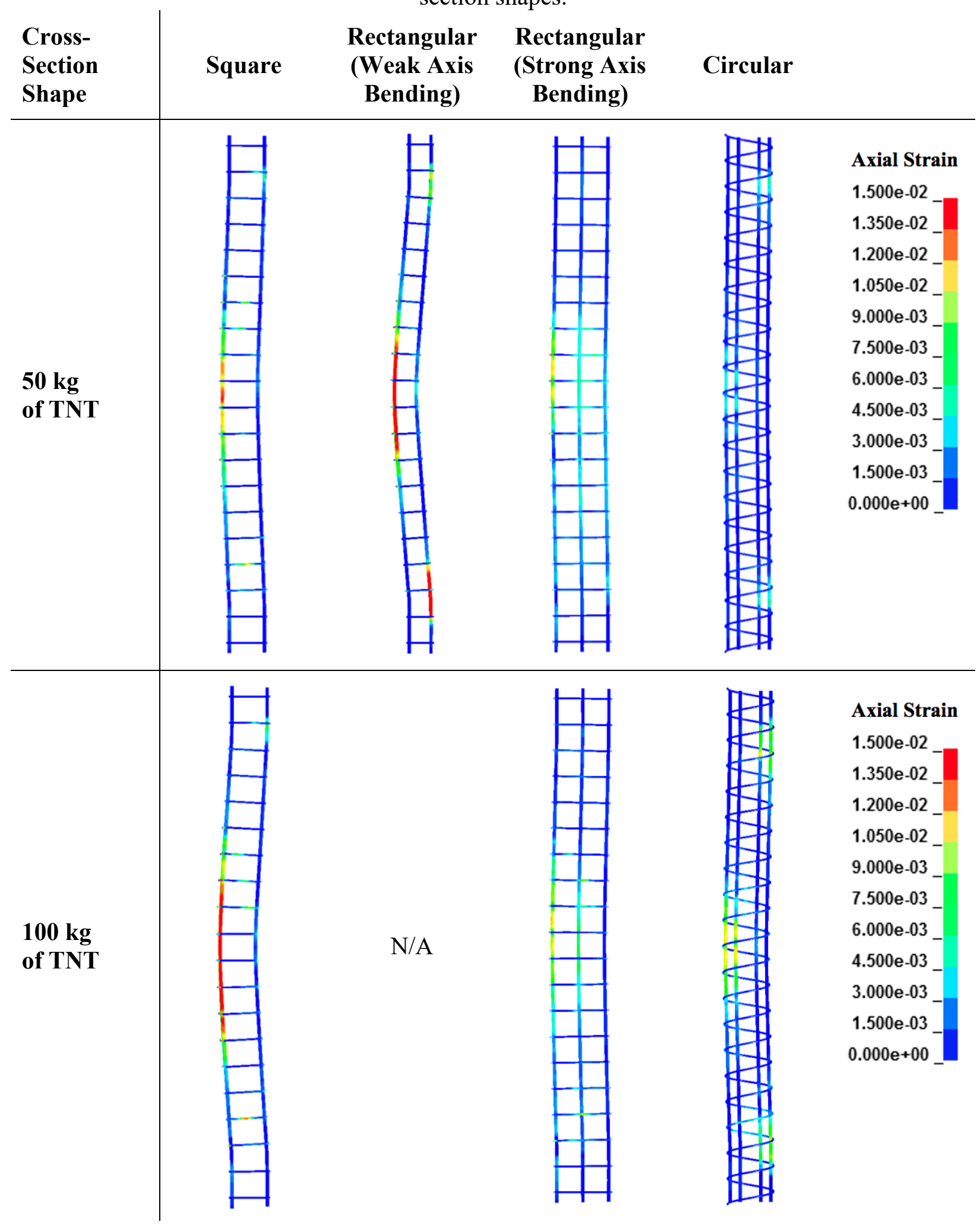




\section{Summary and Conclusions}

This research study presents an investigation into the behaviour of RC columns under near-field blast loads at scaled distances less than $0.4 \mathrm{~m} / \mathrm{kg}^{1 / 3}$. The CFD modelling method was used in LSDYNA to simulate the close-in detonation process of the explosive, its propagation, and its interaction with the structure. A two-phased validation study was performed to evaluate the accuracy of the model against experimental tests available in the research literature. First, the blast pressures computed under near-field explosions were verified against experimentally measured pressure values. Then, the structural response of two RC slab specimens with different damage modes and an RC beam specimen all tested under near-field blast loadings were analyzed. After verification of the CFD model, a series of numerical simulations were conducted to analyze the effect of transverse reinforcement ratio, concrete cover, axial load ratio, and column shape. Two loading scenarios were considered to evaluate column response to different charge masses at the same scaled distance. Based on the results of the verification studies and numerical parametric simulations, the following conclusions were drawn.

- Simplified methods of analysis provide a reliable and efficient means of prediction for blast loading in the far-field. However, they are not applicable for scaled distances below 0.4 $\mathrm{m} / \mathrm{kg}^{1 / 3}$. Instead, CFD should be used to accurately model the detonation, propagation, and interaction of the blast load with the structure. Furthermore, charge mass shape is an important parameter in the accurate prediction of blast pressures in the near-field. As demonstrated in this study, the simplified methods of analysis, such as LBE, do not provide an option to model different charge shapes, and therefore cannot be used to accurately simulate the effects of different charge shapes. 
- Transverse reinforcement spacing has a significant influence on the global and local performance of RC columns under near-field blast loading. With respect to the global response, a reduction in spacing resulted in a reduction in maximum and residual displacements. The failure mode and damage pattern were also greatly affected by the amount of transverse reinforcement. Inadequate transverse reinforcement can shift the failure mode from flexural failure at the mid-height of the column to shear failure near the support. Furthermore, reduction of transverse reinforcement spacing reduced length of spalling on the distal face of the column and also increased the residual axial capacity. Both these performance measures appeared to be affected by local damage incurred during the blast loading.

- The reduction in transverse reinforcement spacing was highly effective in improving both global and local responses of the column when the spacing was greater than half of the cross-section dimension. This was consistent with the design practice in which half of the cross-section dimension is typically considered as a good estimate for spacing of transverse reinforcement. For spacing values below this limit, the amount of transverse reinforcement had negligible influence on the maximum and residual displacements, but still was highly beneficial in controlling the local damage and improving the residual axial capacity.

- The concrete cover had a minor effect on the global response of the RC columns. Maximum displacements showed minor improvement with reduction of concrete cover, while residual displacements were, for the most part, unaffected. However, the reduction of concrete cover had a significant influence on performance measures affected by the local behaviour of the RC columns. Reduction of concrete cover resulted in significant reduction in the length of spalling. However, concrete cover values greater than $40 \mathrm{~mm}$ resulted in a 
significant decline in residual axial capacity of the column due to the decrease in both the confined area and flexural capacity of the cross-section.

- Axial load ratio had a significant effect on the global response of the columns. Increasing axial load ratio generally resulted in lower maximum and residual lateral displacements. However, for the column used in this study, increasing axial load ratio past approximately 0.3 had a negative effect on column behaviour. This led to increased stress and strain near the base support and eventually led to shear failure of the column. This behaviour was amplified when the charge mass was increased. Axial load ratio also affected the local response of the column. Increasing axial load ratio reduced the extent of spalling in concrete. However, the residual axial capacity showed a similar trend to the displacements. Residual axial capacity decreased when the axial load ratio was increased past 0.3 due to the weakening of the column near the base support as a result of the blast loading.

- Column shape was also observed to have an effect on behaviour under blast loading. Circular columns performed significantly better than square or rectangular columns. This was true for both maximum and residual displacements as well as the length of spalling. Due to the geometry of the circular column, the angle of incidence to a point on the front face of the circular column will be greater than that of the square or rectangular column, resulting in lower reflected pressures. The rectangular and square columns also appeared to be affected by depth. The rectangular column with bending about the strong axis resulted in significantly lower displacements which were comparable to that of the circular column. It also resulted in a lower spall length on the distal face of the column.

Considering the lack of experimental studies available for RC columns with axial loading under near-field blast loads, the results obtained from this research study will provide a better 
understanding of the behaviour of RC columns under near-field explosions. The CFD modelling technique can provide more accurate analysis of blast loads on structures in the near-field, particularly when the scaled distance is less than $0.4 \mathrm{~m} / \mathrm{kg}^{1 / 3}$. Data compiled through FE modelling, such as the results obtained in this study, can lead to more efficient design of new blast-resistant structures and more accurate blast performance assessment of existing structures. 


\section{References}

ASCE. (2011). Blast protection of buildings. ASCE Standard. https://doi.org/10.1061/(asce)sc.1943-5576.0000183

Baker, W. E., Cox, P. A., Westine, P. S., Kulesz, J. ., \& Strehlow, R. . (1983). Explosion Hazards and Evaluation. Elsevier.

Bao, X., \& Li, B. (2010). Residual strength of blast damaged reinforced concrete columns. International Journal of Impact Engineering. https://doi.org/10.1016/j.ijimpeng.2009.04.003

Baylot, J. T., \& Bevins, T. L. (2007). Effect of responding and failing structural components on the airblast pressures and loads on and inside of the structure. Computers and Structures. https://doi.org/10.1016/j.compstruc.2007.01.001

Bohara, R., Tanapornraweekit, G., \& Tangtermsirikul, S. (2018). Investigation of concrete material models for analysis of seismic behavior of reinforced concrete under reversed cyclic load. Songklanakarin Journal of Science and Technology.

Broadhouse, B. J., \& Neilson, A. J. (1987). Modelling Reinforced Concrete Structures in DYNA3D. DYNA3D User Group Conference, Safety \& Engineering Science Division.

Carlsson, M., \& Kristensson, R. (2012). Strucutral Response with Regard to Explosions - Mode Superposition, Damping, and Curtailment. Lund University.

Castedo, R., Santos, A. P., Alanon, A., Reifarth, C., Chiquito, M., Lopez, L. M., MartínezAlmajano, S., \& Perez-Caldentey, A. (2021). Numerical study and experimental tests on fullscale RC slabs under close-in explosions. Engineering Structures, 231.

Chen, L., Hu, Y., Ren, H., Xiang, H., Zhai, C., \& Fang, Q. (2019). Performances of the RC column under close-in explosion induced by the double-end-initiation explosive cylinder. International Journal of Impact Engineering. 
https://doi.org/10.1016/j.ijimpeng.2019.103326

Codina, R., Ambrosini, D., \& de Borbón, F. (2016). Experimental and numerical study of a RC member under a close-in blast loading. Engineering Structures. https://doi.org/10.1016/j.engstruct.2016.08.035

Coleman, D. (2016). Evaluation of Concrete Modeling in LS-DYNA for Seismic Application. The University of Texas at Austin.

Cormie, D., Shin, J., Whittaker, A. S., \& Aref, A. J. (2014). Air-Blast Effects on Civil Structures. MCEER.

Cui, J., Hao, H., \& Shi, Y. (2017). Discussion on the suitability of concrete constitutive models for high-rate response predictions of RC structures. In International Journal of Impact Engineering. https://doi.org/10.1016/j.ijimpeng.2017.04.003

Cui, J., Shi, Y., Li, Z.-X., \& Chen, L. (2015). Failure Analysis and Damage Assessment of RC Columns under Close-In Explosions. Journal of Performance of Constructed Facilities. https://doi.org/10.1061/(asce)cf.1943-5509.0000766

Day, J. (2009). Guidelines for ALE Modeling in LS-DYNA. LSTC.

Dua, A., Braimah, A., \& Kumar, M. (2020). Experimental and numerical investigation of rectangular reinforced concrete columns under contact explosion effects. Engineering Structures. https://doi.org/10.1016/j.engstruct.2019.109891

Fan, W., \& Yuan, W. C. (2014). Numerical simulation and analytical modeling of pile-supported structures subjected to ship collisions including soil-structure interaction. Ocean Engineering. https://doi.org/10.1016/j.oceaneng.2014.08.011

Figuli, L., Kavický, V., Boc, K., Vidriková, D., \& Jangl, Š. (2008). Analysis of Blast Loaded Structures. Science and Military, 8. 
Hallquist, J. O. (2006). LS-DYNA Theory Manual. Livermore Software Technology Corporation (LSTC).

Hao, H., Hao, Y., Li, J., \& Chen, W. (2016). Review of the current practices in blast-resistant analysis and design of concrete structures. In Advances in Structural Engineering. https://doi.org/10.1177/1369433216656430

Karlos, V., Solomos, G., \& Larcher, M. (2016). Analysis of blast parameters in the near-field for spherical free-air explosions. Publications Office of the European Union.

Kim, S. B., Kim, H. W., \& Yoo, Y. H. (2015). Penetration analysis of projectile with inclined concrete target. The European Physical Journal Conferences.

Langran-Wheeler, C., Tyas, A., Rigby, S., Stephens, C. S., Clarke, S., \& Warren, J. (2017). Characterisation of reflected blast loads in the very near-field from non-spherical explosive charges. The 17th International Symposium on the Interaction of the Effects of Munitions with Structures.

Lapoujade, V., Van Dorsselaer, N., Kevorkian, S., \& Cheval, K. (2010). A Study of Mapping Technique for Air Blast Modeling. 11th International LS-DYNA Users Conference: Blast/Impact.

Li, J., \& Hao, H. (2014). Numerical study of concrete spall damage to blast loads. International Journal of Impact Engineering, 68, 41-55. https://doi.org/10.1016/j.ijimpeng.2014.02.001

Li, J., Hao, H., \& Wu, C. (2017). Numerical study of precast segmental column under blast loads. Engineering Structures. https://doi.org/10.1016/j.engstruct.2016.12.028

Li, J., \& Wu, C. (2018). Damage evaluation of ultra-high performance concrete columns after blast loads. International Journal of Protective Structures, 9(1), 44-64. https://doi.org/10.1177/2041419617743986 
Low, H. Y., \& Hao, H. (2001). Reliability analysis of reinforced concrete slabs under explosive loading. Structural Safety. https://doi.org/10.1016/S0167-4730(01)00011-X

LSTC. (2017a). LS-DYNA Keyword User's Manual (Volume II). Livermore Software Technology Corporation (LSTC).

LSTC. (2017b). LS-DYNA Keyword User's Manual (Volume I). Livermore Software Technology Corporation (LSTC).

Luccioni, B., \& Aráoz, G. (2011). Erosion Criteria for Frictional Materials Under Blast Load. Mecánica Computacional.

Luccioni, B. M., Ambrosini, R. D., \& Danesi, R. F. (2004). Analysis of building collapse under blast loads. Engineering Structures. https://doi.org/10.1016/j.engstruct.2003.08.011

Malvar, L. J., \& Crawford, J. E. (1998). Dynamic Increase Factors for Steel Reinforcing Bars. Proceedings of the Twenty-Eighth DoD Explosives Safety Seminar.

Mander, J. B., Priestley, M. J. N., \& Park, R. (1988). Theoretical Stress-Strain Model for Confined Concrete. Journal of Structural Engineering. https://doi.org/10.1061/(asce)07339445(1988)114:8(1804)

Murray, Y. (2007). Users Manual for LS-DYNA Concrete Material Model 159. Federal Highway Administration.

Murray, Y. D. (2004). Theory and Evaluation of Concrete Material Model 159. 8th International LS-DYNA Users Conference.

PEC, \& BakerRisk. (2008). U.S. Army Corps of Engineers Protective Design Center Technical Report. Methodology Manual for the Single-Degree-of-Freedom Blast Effects Design Spreadsheets.

Rajkumar, D., Senthil, R., Bala Murali Kumar, B., AkshayaGomathi, K., \& Mahesh Velan, S. 
(2020). Numerical Study on Parametric Analysis of Reinforced Concrete Column under Blast Loading. American Society of Civil Engineers, 34(1).

Randers-Pehrson, G., \& Bannister, K. A. (1997). Airblast Loading Model for DYNA2D and DYNA3D.

Rigby, S. E., Tyas, A., Clarke, S. D., Fay, S. D., Reay, J. J., Warren, J. A., Gant, M., \& Elgy, I. (2015). Observations from preliminary experiments on spatial and temporal pressure measurements from near-field free air explosions. International Journal of Protective Structures. https://doi.org/10.1260/2041-4196.6.2.175

Sandler, I. S. (2005). Review of the development of Cap Models for geomaterials. Shock and Vibration. https://doi.org/10.1155/2005/104968

Sherkar, P., Shin, J., Whittaker, A., \& Aref, A. (2016). Influence of Charge Shape and Point of Detonation on Blast-Resistant Design. Journal of Structural Engineering. https://doi.org/10.1061/(asce)st.1943-541x.0001371

Shi, Y., Hao, H., \& Li, Z. X. (2008). Numerical derivation of pressure-impulse diagrams for prediction of RC column damage to blast loads. International Journal of Impact Engineering. https://doi.org/10.1016/j.jimpeng.2007.09.001

Shi Yanchao, Y., Li, Z. X., \& Hao, H. (2010). A new method for progressive collapse analysis of RC frames under blast loading. Engineering Structures. https://doi.org/10.1016/j.engstruct.2010.02.017

Shin, J. (2014). Air-blast effects on civil structures. University of New York.

Souli, M., \& Benson, D. J. (2010). Arbitrary Lagrangian-Eulerian and Fluid-Structure Interaction: Numerical Simulation. ISTE.

Tancreto, J. E. (1975). Effects of charge shape and composition on blast environment. National 
Technical Information Service.

Tang, E. K. C., \& Hao, H. (2010). Numerical simulation of a cable-stayed bridge response to blast loads, Part I: Model development and response calculations. Engineering Structures. https://doi.org/10.1016/j.engstruct.2010.06.007

Thai, D. K., \& Kim, S. E. (2018). Numerical investigation of the damage of RC members subjected to blast loading. Engineering Failure Analysis. https://doi.org/10.1016/j.engfailanal.2018.06.001

Trajkovski, J., Kunc, R., Perenda, J., \& Prebil, I. (2014). Minimum mesh design criteria for blast wave development and structural response - MMALE method. Latin American Journal of Solids and Structures. https://doi.org/10.1590/S1679-78252014001100006

Tyas, A., \& Watson, A. J. (2001). Investigation of frequency domain dispersion correction of pressure bar signals. International Journal of Impact Engineering. https://doi.org/10.1016/S0734-743X(00)00025-7

U. S. Army Corps of Engineers. (2008). Structures to resist the effects of accidental explosions.

Wang, W., Zhang, D., Lu, F., Wang, S. C., \& Tang, F. (2012). Experimental study on scaling the explosion resistance of a one-way square reinforced concrete slab under a close-in blast loading. International Journal of Impact Engineering. https://doi.org/10.1016/j.ijimpeng.2012.03.010

Wang, Z. L., Konietzky, H., \& Huang, R. Y. (2009). Elastic-plastic-hydrodynamic analysis of crater blasting in steel fiber reinforced concrete. Theoretical and Applied Fracture Mechanics. https://doi.org/10.1016/j.tafmec.2009.08.005

Wenzel, A. B., \& Esparza, E. D. (1974). PRESSURE AND IMPULSE MEASUREMENTS CLOSE TO EXPLOSIONS IN AIR AND IN EARTH. ISA Transactions. 
Xiao, W., Andrae, M., \& Gebbeken, N. (2020). Influence of charge shape and point of detonation of high explosive cylinders detonated on ground surface on blast-resistant design. International Journal of Mechanical Sciences. https://doi.org/10.1016/j.ijmecsci.2020.105697

Yang, F., Feng, W., Liu, F., Jing, L., Yuan, B., \& Chen, D. (2019). Experimental and numerical study of rubber concrete slabs with steel reinforcement under close-in blast loading. Construction and Building Materials. https://doi.org/10.1016/j.conbuildmat.2018.11.248

Zhang, D., Yao, S. J., Lu, F., Chen, X. G., Lin, G., Wang, W., \& Lin, Y. (2013). Experimental study on scaling of RC beams under close-in blast loading. Engineering Failure Analysis. https://doi.org/10.1016/j.engfailanal.2013.06.020

Zhu, L., Elwood, K. J., \& Haukaas, T. (2007). Classification and Seismic Safety Evaluation of Existing Reinforced Concrete Columns. Journal of Structural Engineering. https://doi.org/10.1061/(asce)0733-9445(2007)133:9(1316)

Zineddin, M., \& Krauthammer, T. (2007). Dynamic response and behavior of reinforced concrete slabs under impact loading. International Journal of Impact Engineering. https://doi.org/10.1016/j.ijimpeng.2006.10.012 